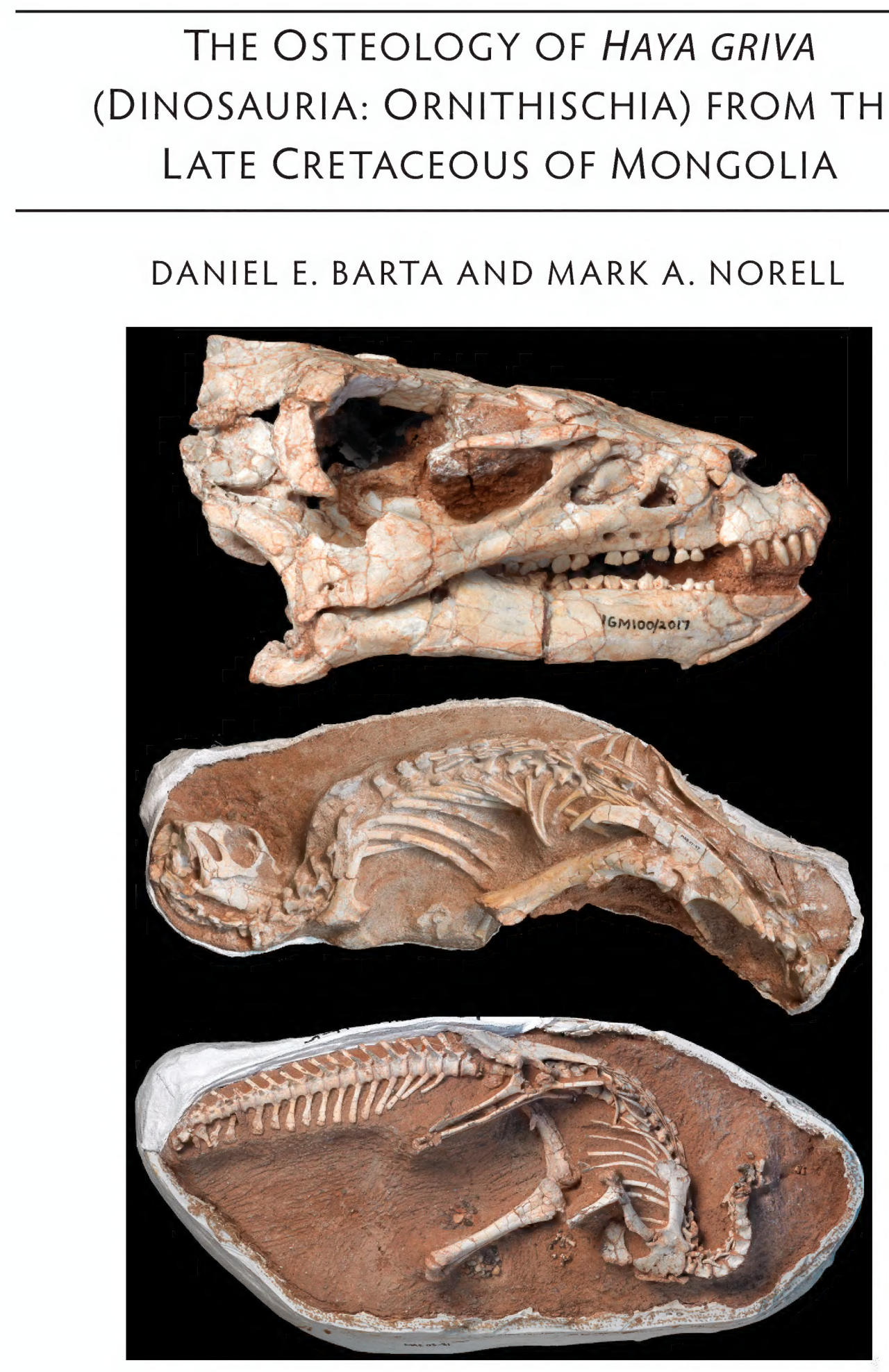

BULLETIN OF THE AMERICAN MUSEUM OF NATURAL HISTORY 


\title{
THE OSTEOLOGY OF HAYA GRIVA \\ (DINOSAURIA: ORNITHISCHIA) \\ FROM THE LATE CRETACEOUS \\ OF MONGOLIA
}

\author{
DANIEL E. BARTA \\ Department of Anatomy and Cell Biology \\ Oklahoma State University College of Osteopathic Medicine \\ at the Cherokee Nation, Tahlequah, OK; and \\ Richard Gilder Graduate School and Division of Paleontology, \\ American Museum of Natural History, New York
}

MARK A. NORELL

Division of Paleontology

American Museum of Natural History, New York

BULLETIN OF THE AMERICAN MUSEUM OF NATURAL HISTORY

Number 445, 111 pp., 58 figures, 1 table

Issued February 26, 2021 


\section{CONTENTS}

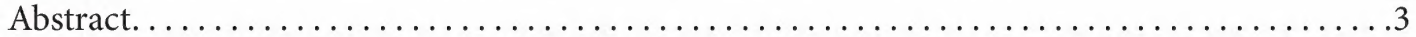

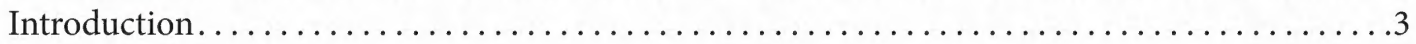

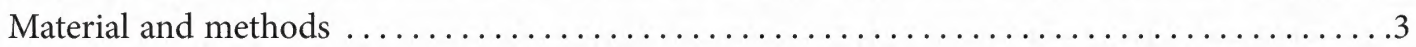

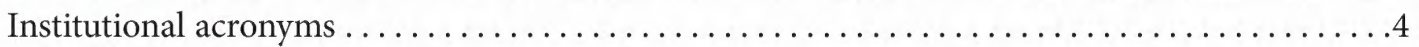

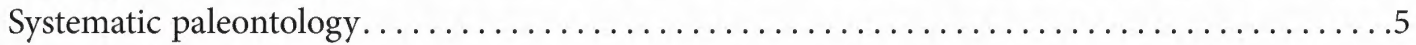

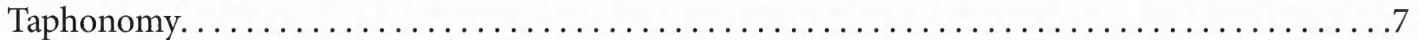

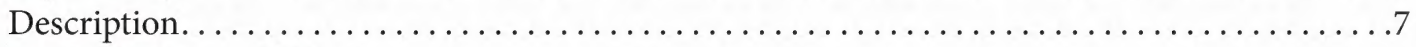

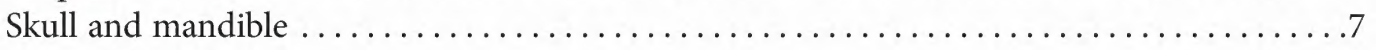

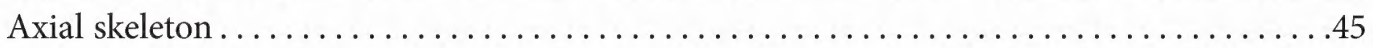

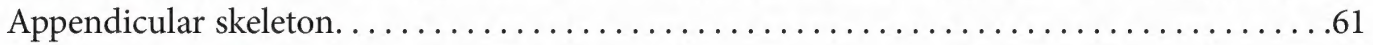

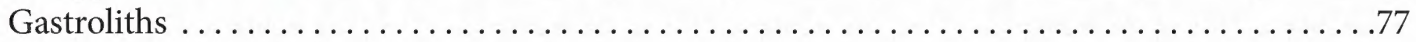

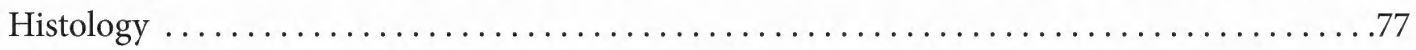

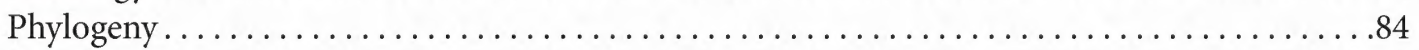

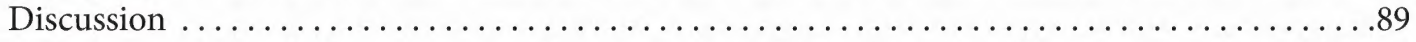

Ontogenetic and other intraspecific variation. ............................ 89

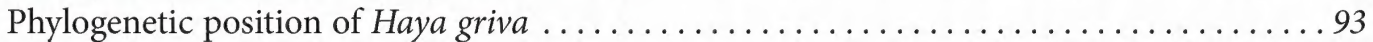

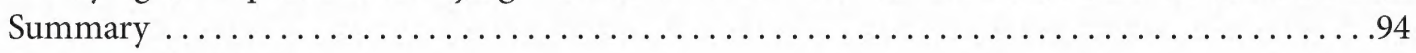

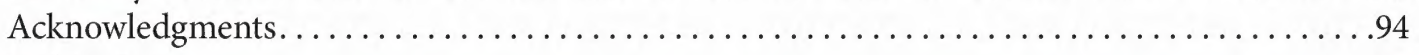

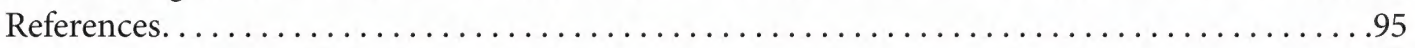

Appendix 1: Anatomical abbreviations ............................ 102

Appendix 2: Character list for phylogenetic analyses . . . . . . . . . . . . . . . . 103

Supplement available online . ................. (https://doi.org/10.5531/sd.sp.47) 


\begin{abstract}
Haya griva is an early-diverging neornithischian ("hypsilophodontid") dinosaur known from several well-preserved skulls and articulated postcranial skeletons, in addition to dozens of partial or isolated finds from the Upper Cretaceous Khugenetslavkant and Zos Canyon localities (Javkhlant Formation and equivalent beds) in the Gobi Desert of Mongolia. Collectively, nearly the entire skeletal anatomy of Haya is known, including partial growth series of skulls and femora. Detailed description and comparisons with other ornithischians, including novel anatomical information about the palate and braincase gleaned through high-resolution x-ray microcomputed tomography, reveals a wealth of osteological data for understanding the growth and relationships of this key taxon. Though the Haya specimens span a wide size range, bone histology reveals that all are likely perinatal to subadult individuals, with specimens of intermediate age the most common, and skeletally mature specimens absent. Phylogenetic analyses place Haya as one of the few Asian members of Thescelosauridae, an important noncerapodan neornithischian group of the Late Cretaceous.
\end{abstract}

\section{INTRODUCTION}

The ornithischian dinosaur Haya griva holds great evolutionary, systematic, biostratigraphic, and paleoecological significance. It is the only representative of an early-diverging ornithischian dinosaur currently known among the vast diversity of Mesozoic vertebrates from Mongolia's Gobi Desert. Despite previous expeditions to the Upper Cretaceous (Santonian-Campanian) eastern Gobi exposures of the Javkhlant Formation (Shine Us Khudag redbeds) at the Khugenetslavkant locality (Eberth et al., 2009; fig. 1) by Russian, Mongolian, and American paleontologists, the presence of a primitive neornithischian dinosaur at this site was not revealed until the first discovery of its fossils in 2002 by the joint Mongolian Academy of Sciences-American Museum of Natural History expedition (Makovicky et al., 2011). Makovicky et al. (2011) named and described Haya griva on the basis of an articulated holotype skull and several referred specimens consisting of articulated and partially articulated cranial and postcranial remains. We extensively describe these materials and another specimen (IGM 100/3181) first presented by Norell and Barta (2016). We also describe six new nearly complete or partial specimens of Haya griva, and compare them to other ornithischian dinosaurs, particularly those for which excellent material also exists. Such comparisons enhance com- parative anatomical knowledge of early-diverging neornithischians. The combination of character states described in Haya provides further information for attempts to resolve the unstable relationships and complex, mosaic distribution of characters within clades along this grade (Boyd, 2015). In order to provide a thorough account of the morphology, growth, systematics, and probable life habits of Haya griva, we apply high-resolution X-ray microcomputed tomography $(\mu \mathrm{CT})$ scanning and bone histology to a partial growth series of this taxon for the first time, reassess its phylogenetic affinities, and further evaluate the implications of gastroliths associated with three specimens.

\section{MATERIAL AND METHODS}

The holotype and referred specimens collected by the Mongolian Academy of SciencesAmerican Museum of Natural History (MAE) expeditions listed below form the basis of this description.

Four Haya griva skulls (IGM 100/2016, IGM 100/2017, IGM 100/3178, IGM 100/3181) were scanned using high resolution $\mathrm{X}$-ray microcomputed tomography $(\mu \mathrm{CT})$ at the American Museum of Natural History Microscopy and Imaging Facility (MIF) with a GE v|tome|x CT scanner at $160 \mathrm{kv}$, with a $0.5 \mathrm{~mm}$ copper filter, a current of $200 \mu \mathrm{A}$, and a voxel dimension of $0.04 \mathrm{~mm}$ to produce 1800 images. All of these 


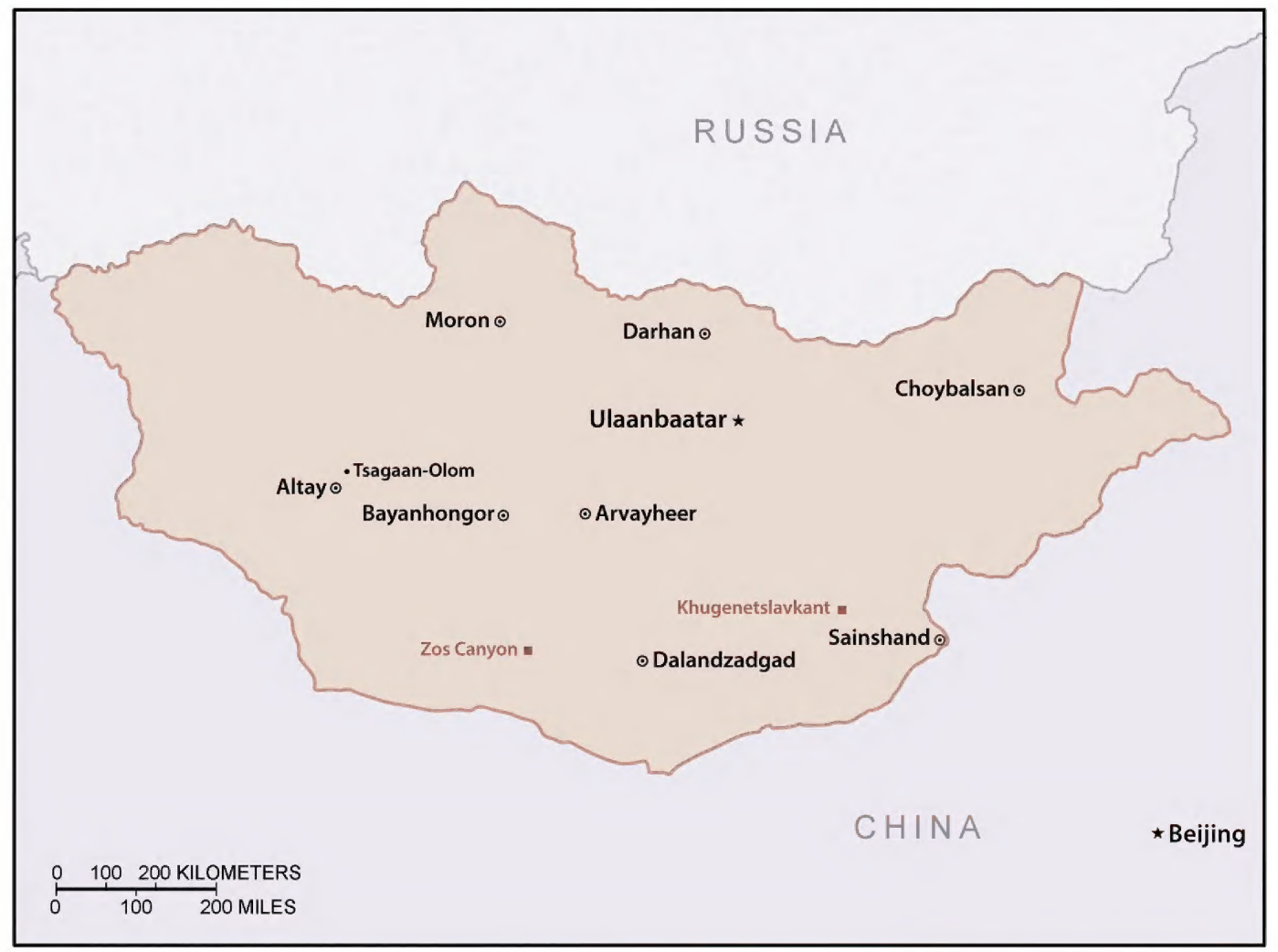

FIG. 1. Map of Mongolia showing the Khugenetslavkant and Zos Canyon localities.

except IGM 100/3181, which was not processed for this study, were reconstructed using Phoenix Datos X (GE Inspection Technologies, LP, Lewistown PA) and FIJI (ImageJ, Schindelin et al., 2012) software, and features of interest were segmented with VGStudio Max (Volume Graphics, Inc. Charlotte, NC). Key specimens (IGM 100/1324, IGM 100/2014, IGM 100/2015, IGM 100/2016, IGM 100/2017, IGM 100/2019, IGM 100/2020, IGM 100/3137, IGM 100/3178, IGM 100/3181, and IGM 100/3661) were surface scanned with a Space Spider surface scanner (Artec, Luxemborg). Stereolithography (STL) files of these specimens are provided as supplementary information available online (https://doi.org/10.5531/sd.sp.47). Bone histology and phylogenetic analysis methods are described in the relevant sections below.

\section{INSTITUTIONAL ACRONYMS}

AMNH FARB American Museum of Natural History Fossil Amphibians, Reptiles, and Birds Collection

BYU Earth Sciences Museum, Brigham Young University, Provo, Utah

IGM Institute of Geology, Mongolian Academy of Sciences, Ulaan Baatar, Mongolia

ROM Royal Ontario Museum, Toronto, Ontario, Canada

MOR Museum of the Rockies, Bozeman, Montana

YPM Yale Peabody Museum of Natural History, New Haven, Connecticut 


\section{SYSTEMATIC PALEONTOLOGY}

Dinosauria Owen, 1842

Ornithischia Seeley, 1887

Neornithischia Cooper, 1985 (sensu Sereno, 1998)

Haya Makovicky et al., 2011

Haya griva Makovicky et al., 2011

Etymology: "Haya griva, from the Sanskrit for the Hindu deity Hayagriva, an avatar of Vishnu characterized by a horse head, in reference to the elongate and faintly horselike skull of this dinosaur, and the common depiction of this deity in the Buddhist art of Mongolia" (fig. 2) (Makovicky et al., 2011: 626).

Holotype: IGM 100/2017 (figs. 3-5, 17A, 18A, 21-26A, 30), complete articulated skull (CT scanned) and anterior cervical vertebrae.

Referred Specimens:

IGM 100/1324 (figs. 41, 51, 52), large isolated femur (the largest in the studied sample).

IGM 100/2013 (figs. 44, 47), "dorsal rib and series of chevrons, an articulated right crus and pes, part of left pes" (Makovicky et al., 2011: 626) and right femur.

IGM 100/2014 (figs. 9, 17B, 26B, 20), Largely complete crushed skull articulated with cervical vertebrae.

IGM 100/2015 (figs. 27, 31, 35, 37, 38, 42, 49, 51, 52), Articulated postcranial skeleton; a cast is catalogued as AMNH FARB 30635.

IGM 100/2016 (fig. 10), largely complete articulated skull (CT scanned).

IGM 100/2018 (fig. 11), anterior portion of small skull.

IGM 100/2019 (figs. 6, 18B, 19, 28, 33, 34), largely complete skull and articulated postcrania.

IGM 100/2020 (figs. 43, 51, 52), postcranial fragments including ribs, femur, tibia, and pedal phalanges (Makovicky et al., 2011: 626). This specimen includes the smallest femur in the studied sample.
IGM 100/3137 (fig. 29), articulated postcrania lacking skull.

IGM 100/3178 (figs. 7, 32, 36, 39, 40, 45, 46, 48), skull (CT scanned) and partially articulated postcrania.

IGM 100/3181 (fig. 8), skull (CT scanned) and articulated dorsal series with ribs, manual phalanges, radius and ulna (Norell and Barta, 2016).

IGM 100/3182 (fig. 50), partial postcrania, including a largely complete left leg, some dorsal vertebrae, and associated gastroliths.

IGM 100/3557 (fig. 12), crushed partial skull.

IGM 100/3661 (fig. 14), disarticulated partial skull and skeleton.

IGM 100/3672 (fig. 15), partial pelvic region and articulated legs and feet from two individuals in close proximity, centra of the axis and third cervical vertebra, a jaw fragment and other surface collected fragments, including a possible atlantal neurapophysis. An isolated troodontid tooth was found in the matrix inside the jacket during preparation (fig. 16).

MAE 15-84, isolated femur.

Many additional specimens (IGM 100/3561, $100 / 3632,100 / 3633,100 / 3634,100 / 1322$, $100 / 1873,100 / 3558,100 / 3559,100 / 3560$, $100 / 3562,100 / 3563,100 / 3564,100 / 3565$, $100 / 3566,100 / 3567,100 / 3568,100 / 3569$, $100 / 3570,100 / 3571,100 / 3604,100 / 3605$, MAE 03-21) (fig. 13) comprise surface-collected cranial and postcranial fragments, including an isolated frontal (IGM 100/3567) and abundant partial maxillae and dentaries. These are all from very small, apparently juvenile (or even perinatesized) individuals. IGM $100 / 3561$ is from the Red Rum sublocality at Zos Canyon (Norell and Barta, 2016).

Locality and Geologic Setting: All specimens other than IGM 100/3181 (Norell and Barta, 2016), IGM 100/3661, and IGM 100/3561 are from the Upper Cretaceous (Santonian-Campanian) Javkhlant Formation at the Khugenetslavkant locality within the Shine Us Khudag badlands in the southeastern Gobi Desert of 


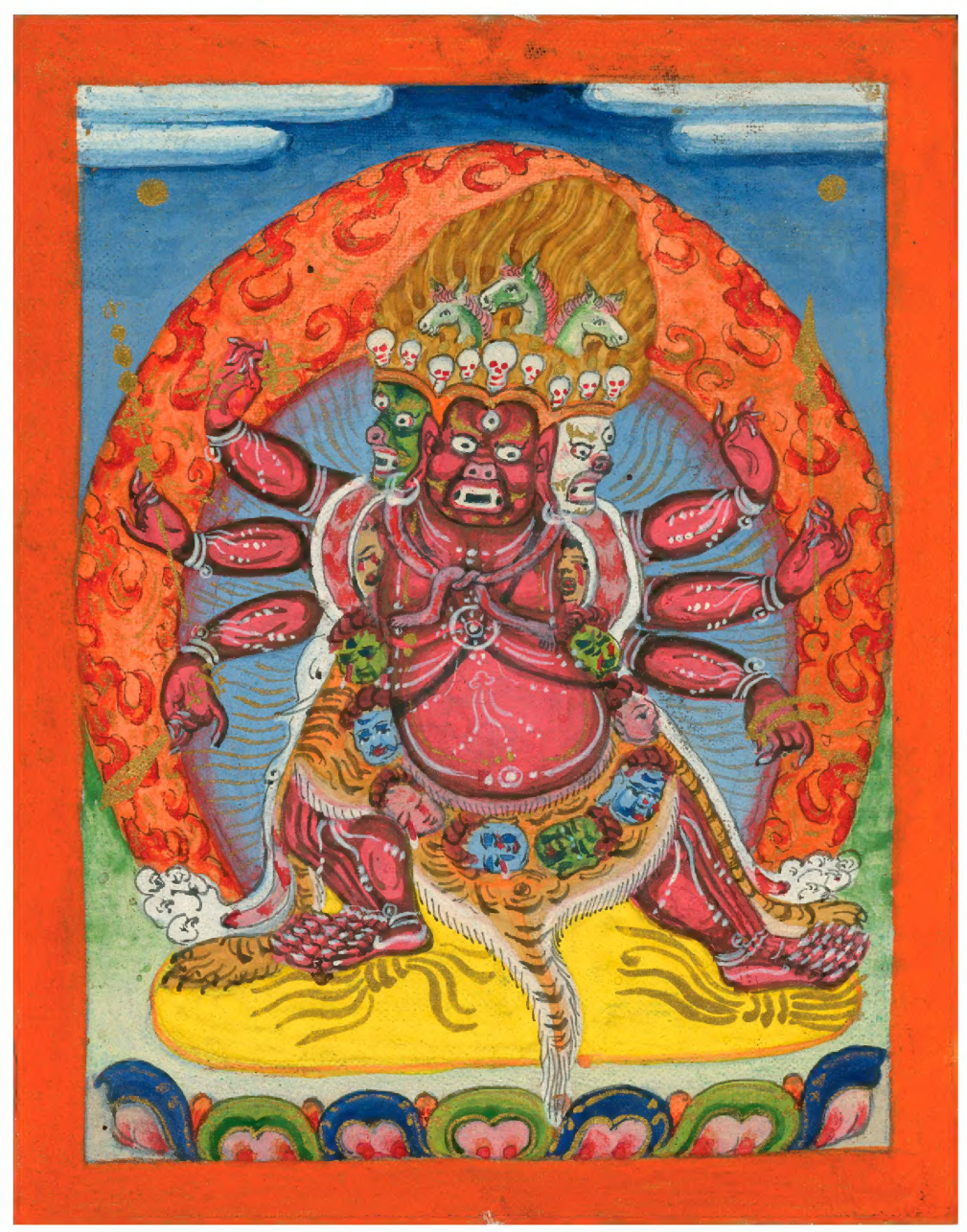

FIG. 2. A Buddhist depiction of the deity Hayagriva.

Mongolia (Eberth et al., 2009; fig. 1). They were found primarily within a $90 \mathrm{~m}$ thick section of the middle Javkhlant beds (Eberth et al., 2009). IGM $100 / 3181$ is from the Zos Canyon locality in the western Gobi, and illustrates the widespread geographic distribution of Haya griva during the Late Cretaceous. The presence of this specimen also provides important evidence for correlating the Javkhlant Formation and Zos Canyon beds, and by extension illustrating the conformable nature of the stratigraphic sequence spanning the base of the Bayn Shire Formation to the top of the Djadokhta Formation (Norell and Barta, 2016).
Emended Diagnosis: Makovicky et al. (2011: $627)$ identified a combination of seven characters diagnostic of Haya griva in their differential diagnosis:

(1) "five homodont, bulbous, unserrated premaxillary teeth"; (2) "premaxilla without rugose ramphothecal pad"; (3) "triangular accessory maxillary fenestra"; (4) "jugal with bifid caudal ramus"; (5) "quadratojugal foramen present"; (6) "midline depression along internasal suture"; and (7) "predentary with bifid ventral ramus."

To these we add an eighth: a bifid anterior process of the jugal (only present in Haya and Heterodontosaurus). Autapomorphies of Haya 
griva resulting directly from a new phylogenetic analysis are discussed in the Phylogeny section below.

\section{TAPHONOMY}

Both the Shine Us Khudag and Zos Canyon localities preserve Haya specimens as articulated, partially articulated, associated, and isolated elements. One of the Zos Canyon specimens, IGM $100 / 3661$ (fig. 14), is unusual in that the skull and postcranial bones are all fairly widely separated from one another, possibly dispersed by scavengers or running water. Articulated specimens (e.g., IGM 100/2015) are contorted in a death pose typical of small ornithischians (Santa Luca, 1980; Zheng et al., 2012), in which the back is strongly flexed, the neck curled back, and the knees bent slightly forward (figs. 27-29). Articulated postcranial specimens often lack the hands, distal half of the tail, and sometimes the skull. When information from all specimens is combined, however, only some of the manual elements, distalmost tail, and a few braincase elements remain unknown or too poorly preserved to describe.

IGM $100 / 3672$ is unusual in that it contains the remains of two individuals, the pelvic region and both legs of one, and a foot from a third that points opposite to the other two feet (fig. 15). This is the only specimen that preserves definitive evidence of two Haya buried in close proximity to one another. A troodontid tooth also was recovered from matrix within the same jacket as IGM $100 / 3672$ (fig. 16). To our knowledge, this is the first published indication of troodontids from the Javkhlant Formation. Many Haya bones contain holes, likely made by insects feeding on the bones postmortem, as in many other Gobi specimens (Norell and Makovicky, 1997; Fanti et al., 2012; Norell et al., 2018). None of the Haya specimens have been recovered from any kind of burrow structure, in contrast to the related Oryctodromeus (Varricchio et al., 2007).

\section{DESCRIPTION}

\section{Skull ANd Mandible}

The following description is based primarily on elements as preserved in the Haya griva holotype, IGM 100/2017 (figs. 3-5, 17A, 18A, 21-26). Elements not preserved or readily visible in IGM $100 / 2017$ are described from other specimens in which they are visible. Morphological differences between IGM 100/2017 and other specimens also are emphasized to comprehensively document variation within Haya griva. Definitions of clade names follow those provided by Madzia et al. (2018).

Premaxilla and Premaxillary Dentition: The anterior margin of the premaxilla overhangs the anteriormost premaxillary tooth, though it is not developed into a hooked projection to the extent seen in Thescelosaurus, Changchunsaurus, and Hypsilophodon (Huxley, 1870; Galton, 1974a; Jin et al., 2010; Boyd, 2014). The two nutrient foramina on the anteriormost portion of the premaxilla form a "figure 8" configuration, as noted by Makovicky et al. (2011). Just posterior to the nutrient foramina, at the position of the first tooth, the premaxilla bulges laterally. The posterior portion of the premaxilla laterally flares and thins dorsally along the subnarial process, forming a flat articular surface with the lateral side of the nasal (fig. 3, 5). The premaxilla of Haya griva is proportionally dorsoventrally taller and anteroposteriorly shorter than that of Thescelosaurus (Boyd, 2014) and has a more dorsally directed subnarial process. As in Hypsilophodon, Isaberrysaura, and possibly Orodromeus, the subnarial process of Haya gently curves posteriorly, but does not contact the lacrimal (Galton, 1974a; Scheetz, 1999; Makovicky et al., 2011; Salgado et al., 2017). The process is taller, narrower, and less anteroposteriorly expanded than the condition seen in Jeholosaurus (Barrett and Han, 2009). The supranarial (ascending) process of the premaxilla is incompletely preserved in all Haya specimens, with only a short process remaining (figs. 3, 4, 5, $7,8,11)$. The anteriormost points of the nasals opposite the external nares from the ascending 


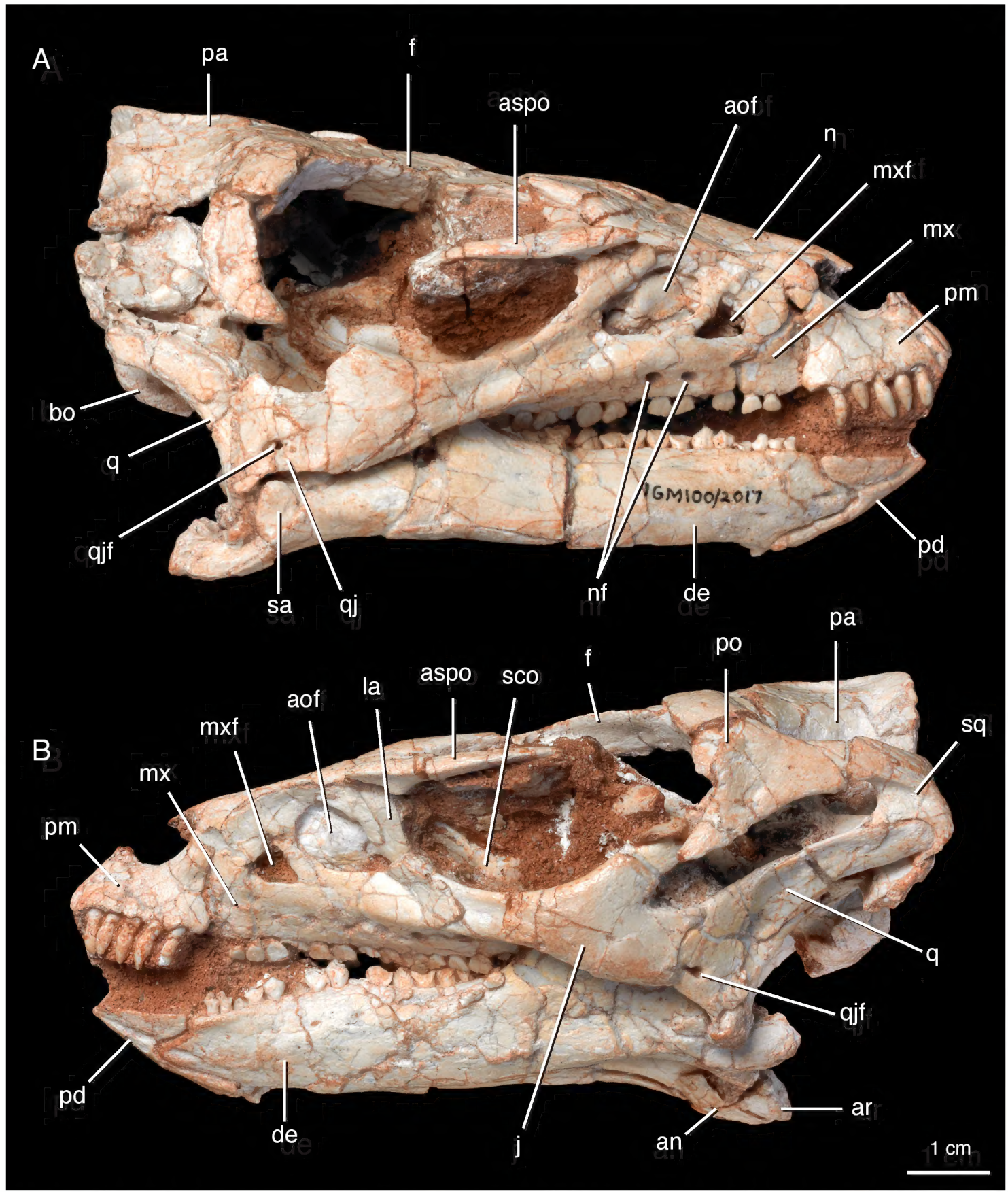

FIG. 3. Skull of the holotype specimen of Haya griva, IGM 100/2017, in A, C, right lateral and B, D, left lateral views. Abbreviations in appendix 1. 

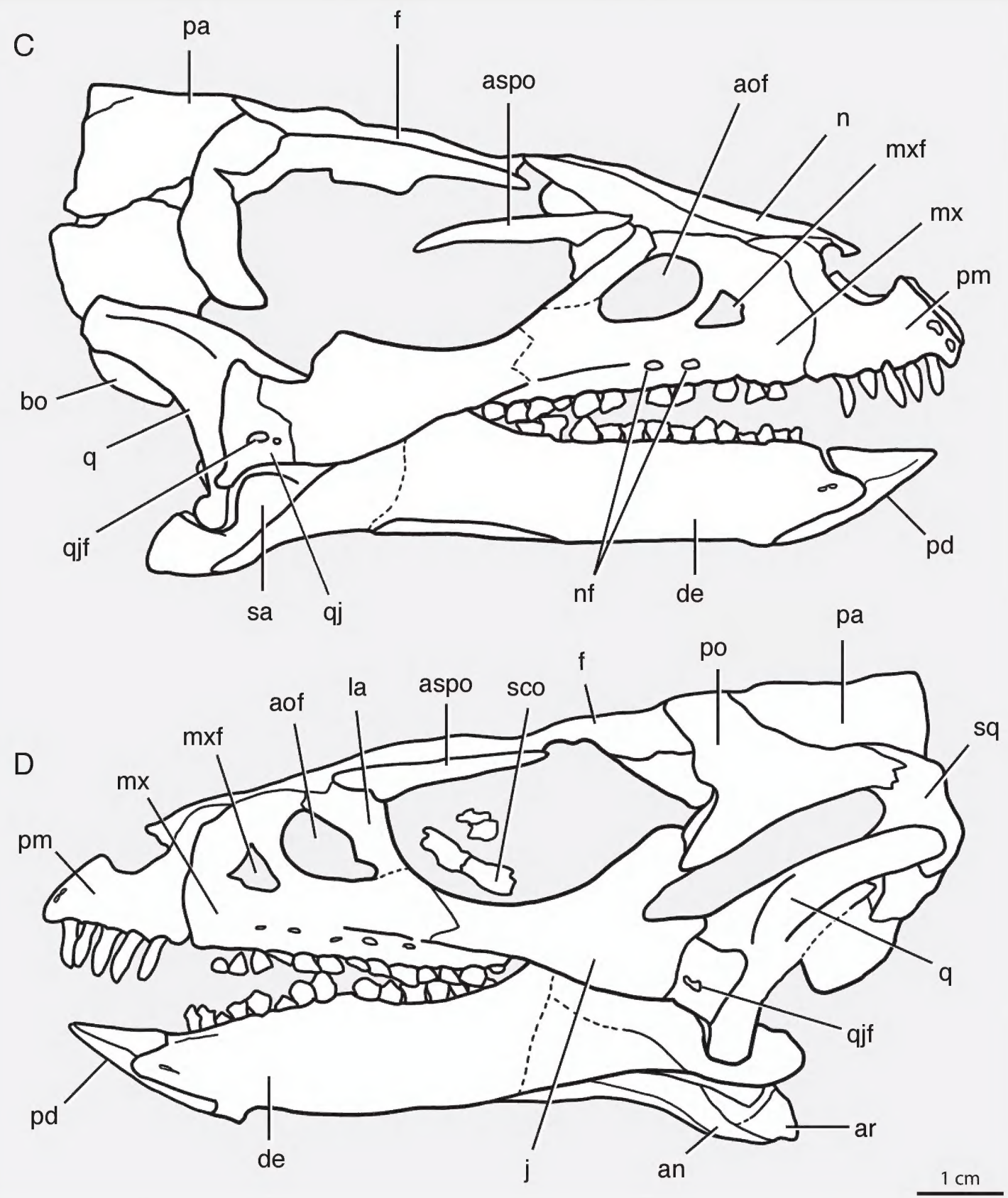


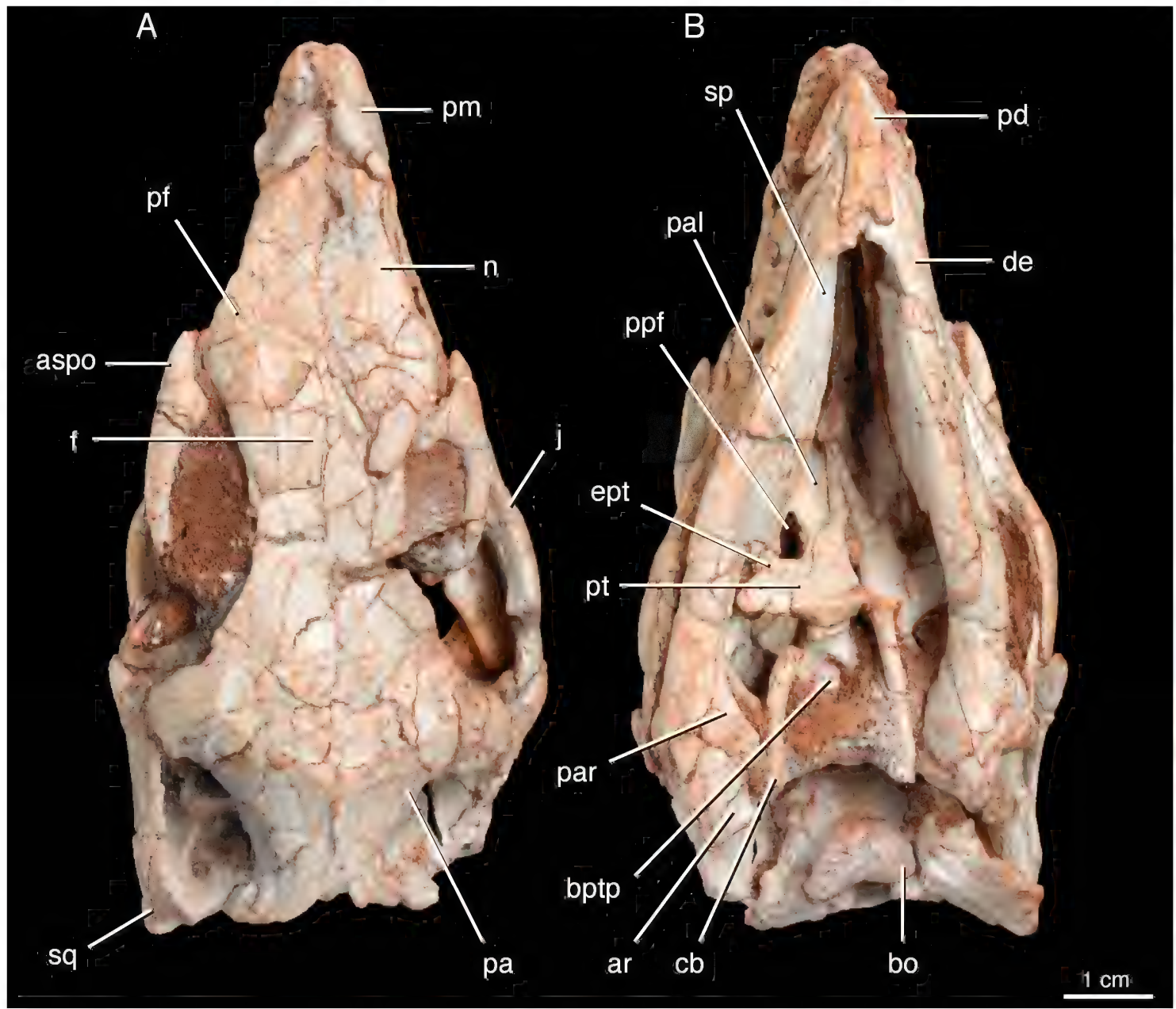

FIG. 4. Skull of the holotype specimen of Haya griva, IGM 100/2017, in A, C, dorsal and B, D, ventral views. Abbreviations in appendix 1.

processes of the premaxillae appear damaged, providing no evidence about the morphology of the internarial bar. The apparently confluent nature of the external nares of Haya (fig. 5) is best considered a taphonomic artifact because confluent external nares (i.e., with loss of a complete internarial bar) are unknown among dinosaurs except for some diplodocoid and titanosaur sauropods (Upchurch, 1995; Wilson et al., 2016). In Haya, the surface of the premaxilla is smooth, in contrast to the rugose anterior premaxillary surfaces of Lesothosaurus, Jeholosaurus, Changchunsaurus, Oryctodromeus, Zephyrosaurus, Thescelosaurus, and Hypsilophodon (Galton, 1974a; Sues, 1980; Sereno, 1991; Varricchio et al., 2007; Barrett and Han, 2009; Jin et al., 2010; Boyd, 2014). Such rugose surfaces have been interpreted as evidence of a ramphotheca (Galton, 1974a), but the extent and attachment of a ramphotheca in Haya, if present, is unclear. The general morphology of the premaxilla anterior to the first premaxillary tooth is similar to Lesothosaurus (Sereno, 1991; Knoll, 2008), except Haya lacks even the limited pitting inferred to correspond to a short ramphotheca anterior to the first premaxillary tooth in Lesothosaurus (Sereno, 1991; Knoll, 2008). Future discoveries of morphologically mature skulls may reveal whether pitting 


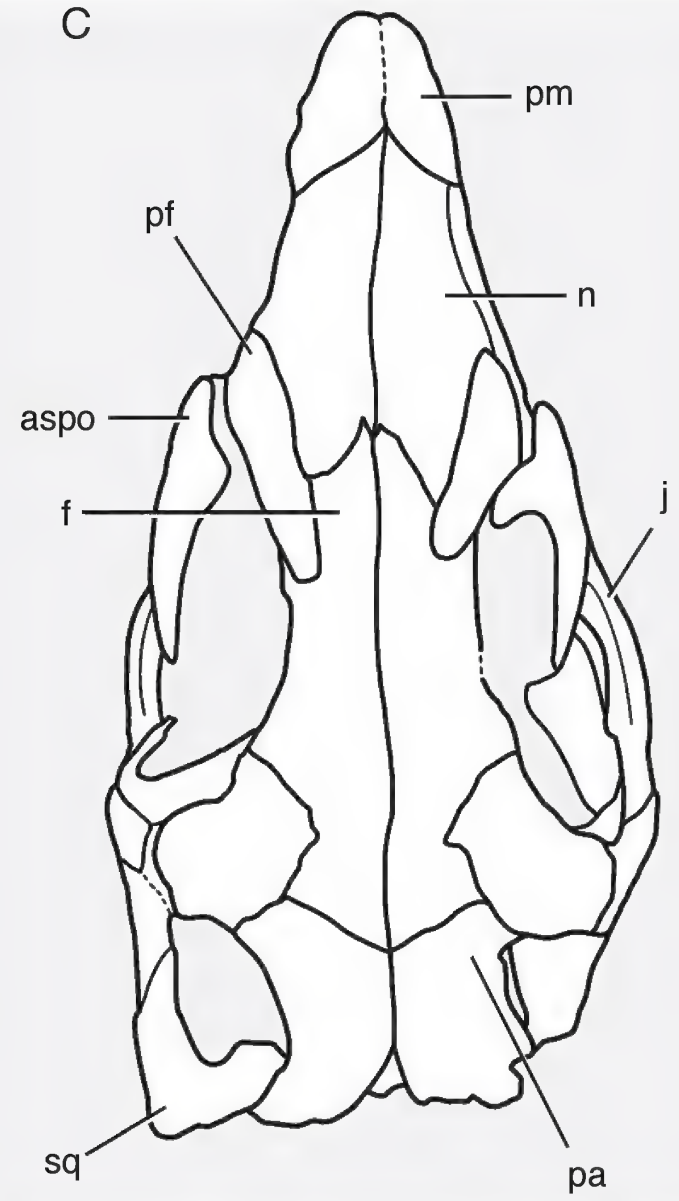

and, by inference, a ramphotheca, developed on the premaxilla of Haya griva through ontogeny, as in Jeholosaurus (Barrett and Han, 2009). In IGM 100/2017, the premaxillae are unfused along their midline contact. Many other primitive ornithischian taxa, including Oryctodromeus, Changchunsaurus, and Thescelosaurus, possess fused or partially fused premaxillae (Norman et al., 2004; Varricchio et al., 2007; Jin et al., 2010; Boyd, 2014). The premaxilla contains five teeth, as is typical for most early-diverging ornithischians (Norman et al., 2004), though six are present in Lesothosaurus, Isaberrysaura, Jeholosaurus, and Thescelosaurus (Sereno, 1991; Barrett and Han, 2009; Boyd, 2014; Salgado et al., 2017). Boyd (2014) noted that the number of premaxillary teeth may have increased through ontogeny in

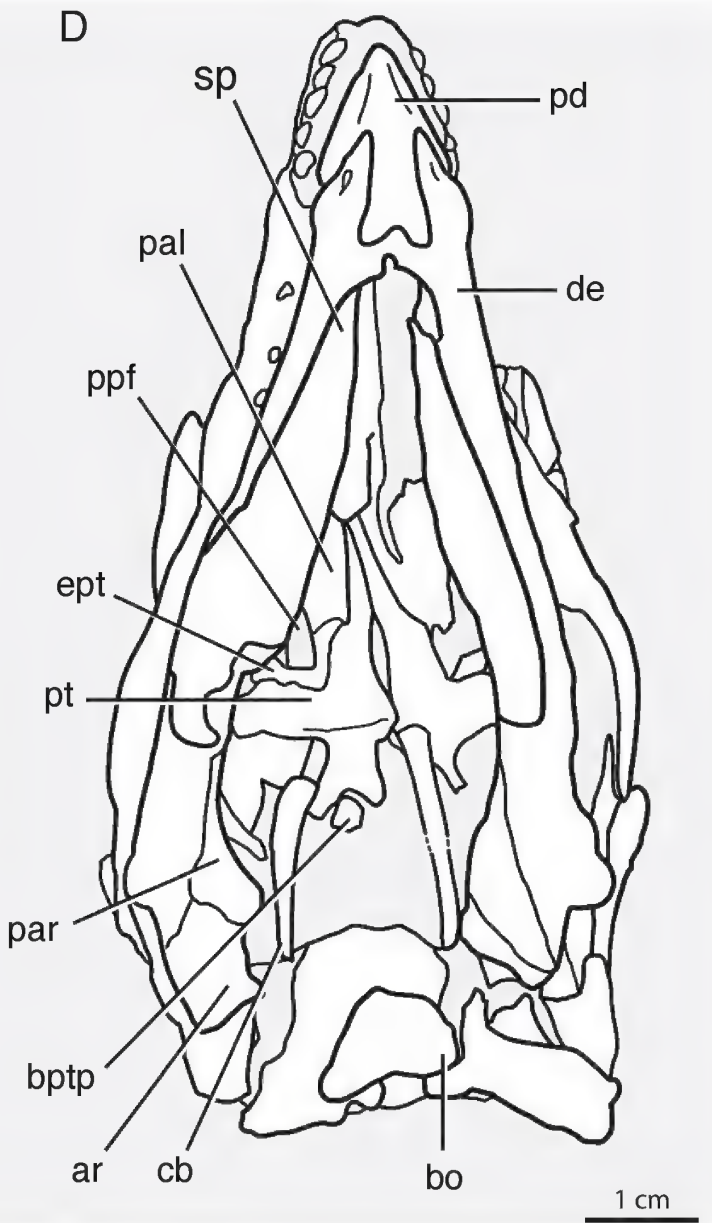

Jeholosaurus and Thescelosaurus, based on his personal observations of multiple specimens of the two taxa. So far, there is no evidence for an increase in premaxillary tooth count through ontogeny in Haya, but future discoveries of ontogenetically more mature specimens are needed to test this. The teeth lack serrations (Makovicky et al., 2011) but bear very faint longitudinal striations. As in Lesothosaurus, the premaxillary teeth lack wear facets, suggesting that during feeding they worked in opposition to the softer keratinous beak inferred to be present on the predentary (Knoll, 2008; but Norman et al., 2004, notes the presence of premaxillary tooth wear facets in other taxa). The apicalmost portion of each tooth is slightly recurved (figs. 3, 7, 8). In IGM $100 / 2017$, the first and third right premaxillary 


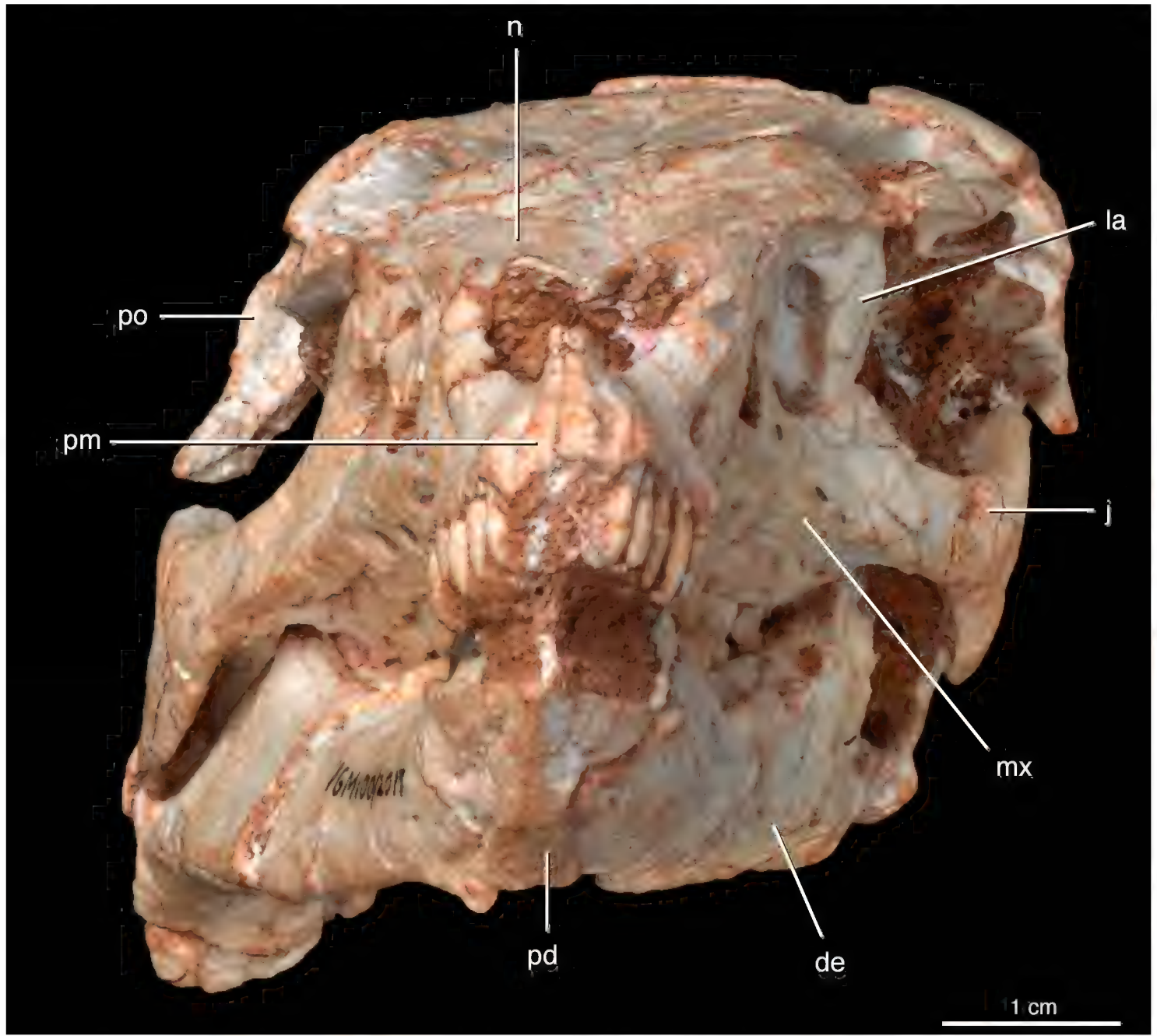

FIG. 5. Skull of the holotype specimen of Haya griva, IGM 100/2017, in anterior view. Abbreviations in appendix 1 .

teeth are somewhat bulbous, unlike the more pointed second, fourth, and fifth teeth. This is also true of the left premaxillary teeth, though the tips of the second and fifth teeth are broken on this side. The first and third left premaxillary teeth of IGM 100/3178 (fig. 7) are also somewhat bulbous, but the other premaxillary teeth are largely obscured. IGM 100/2018 (fig. 11) is not well preserved, but the left premaxilla is more complete than the right. The suture between the two premaxillae is visible at the anteriormost end of the rostrum, and the quality of preservation makes it difficult to tell whether or not the two bones are fused. The premaxillary toothrow is in line with the maxillary toothrow, in contrast to Hypsilophodon, where they are offset (Galton, 1974a; Makovicky et al., 2011). These two toothrows are separated by a diastema, as is present in most basal neornithischians (Norman et al., 2004; Makovicky et al., 2011). It is unclear whether the diastema present in these taxa is homologous to that of theropods or the possible sauropodomorph Eoraptor (Langer and Benton, 2006; Sereno et al., 2012). 


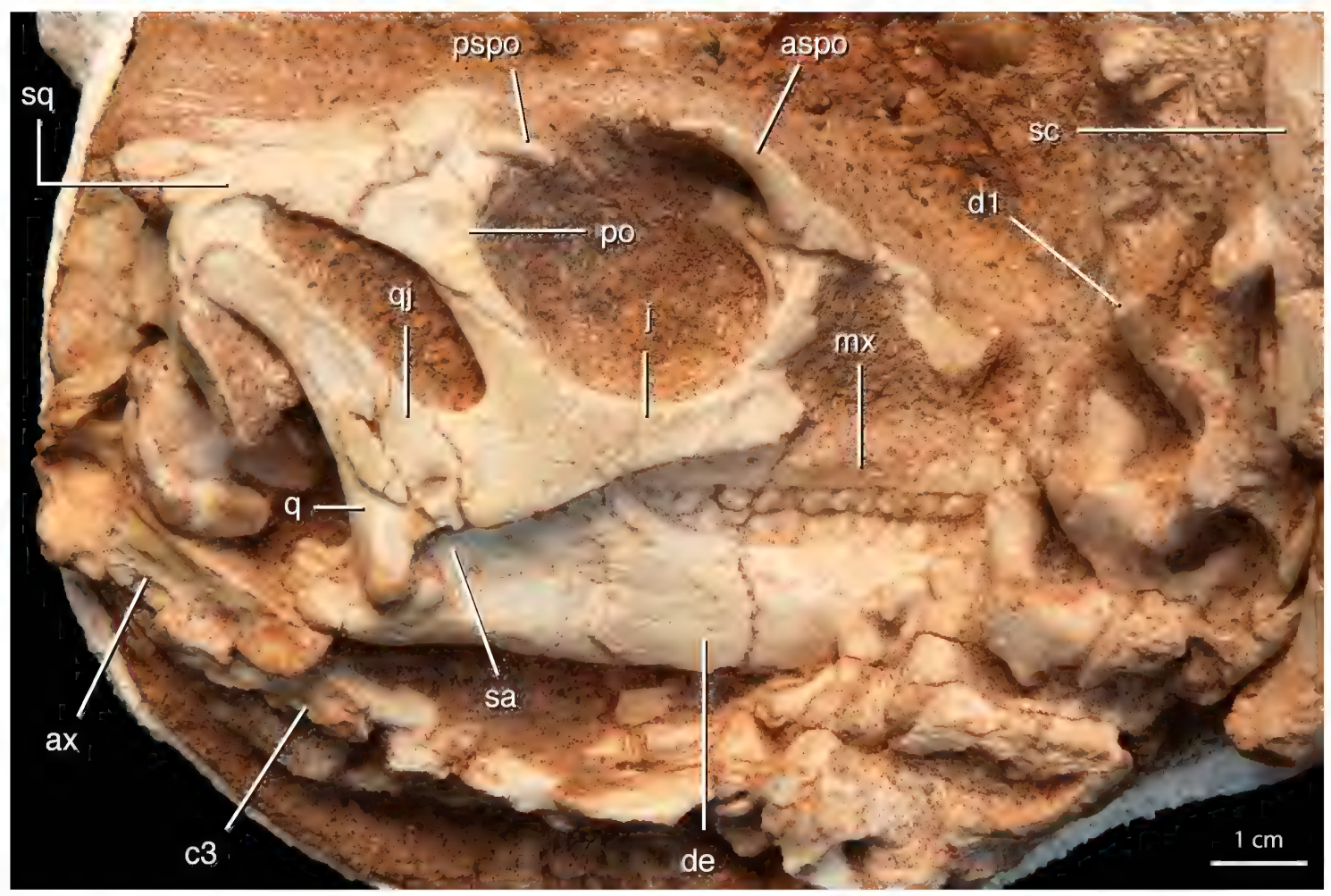

FIG. 6. Skull and cervical vertebrae of Haya griva, IGM 100/2019, in right lateral view (skull) and dorsolateral view (vertebrae). Abbreviations in appendix 1.

Maxilla and Maxillary Dentition: The maxilla contacts the premaxilla anteriorly, a small portion of the nasal dorsally, the lacrimal and jugal posterolaterally, the palatine medially, and the ectopterygoid posteromedially. In palatal view, it forms the lateral border of the postpalatine (suborbital) fenestra as in Heterodontosaurus, Changchunsaurus, and Hypsilophodon (Galton, 1974a; Jin et al., 2010; Norman et al., 2011; fig. 4B, D). The ramus of the maxilla is straight in lateral view in Haya griva. In Thescelosaurus, the posterior end of the ramus curves dorsally. The maxilla of Haya becomes mediolaterally thin dorsally where it meets the nasal and premaxilla. A narrow gap between the premaxilla and maxilla, just dorsal to the diastema between the premaxillary and maxillary toothrows, forms the anterior maxillary fossa (Boyd, 2014). The long axis of this narrow fossa is oriented dorsoventrally in Haya, in contrast to the round fossa of Thescelosaurus. The toothrow is strongly inset from the rest of the maxilla, with a well-defined emargination (the buccal ridge) between the two. In lateral view, the buccal ridge dips ventrally and continues posteriorly to below the midpoint of the orbit (Makovicky et al. 2011). At least six nutrient foramina lie ventral and parallel to the buccal ridge in IGM 100/2017, with the more posterior three being larger than the anterior three. The anterior three nutrient foramina face laterally, but the posterior three face more lateroventrally underneath the overhanging buccal ridge. There are five or six foramina in IGM 100/3181, four small anterior ones, one larger posterior foramen, and possibly one shallow ventrally facing foramen near the junction with the jugal. At least four nutrient foramina pierce the incomplete right maxilla of IGM 100/2014; however, the two posteriormost foramina are smaller than the anterior two. Two 


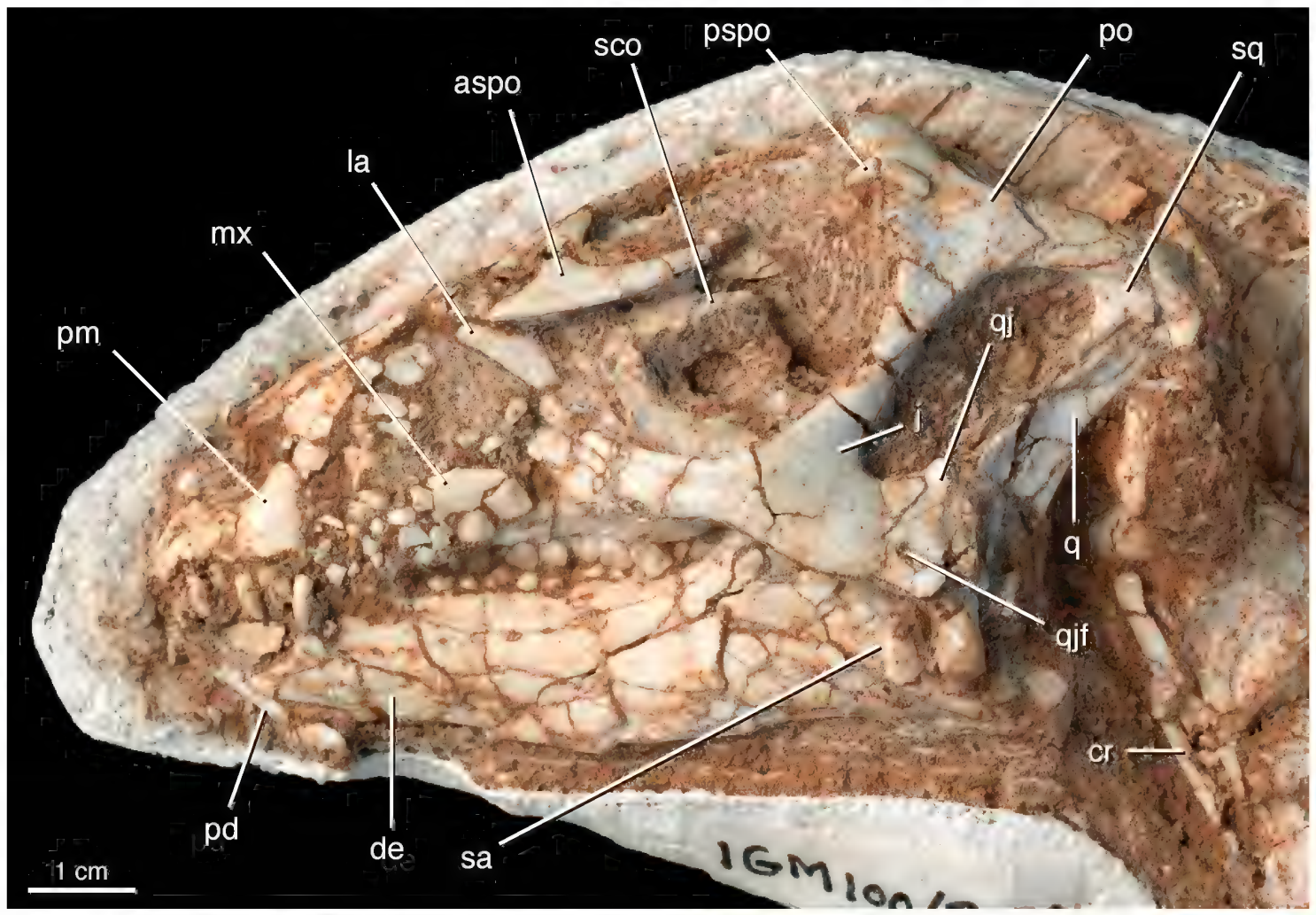

FIG. 7. Skull of Haya griva, IGM 100/3178, in left lateral view. Abbreviations in appendix 1.

foramina are present on the anteriorly eroded maxilla of IGM $100 / 2016$. Thus, it is clear that the relative sizes and possibly the number of these formina are variable in Haya griva. Haya lacks the row of smaller foramina on the lateral surface of the buccal ridge seen in Thescelosaurus (Boyd, 2014). A triangular maxillary fenestra lies anterior to the antorbital fenestra, and is separated from it by a narrow, posteriorly concave interfenestral strut (figs. 3, 8). In Haya, the antorbital fenestra is larger than the maxillary fenestra. Among other early-diverging neornithschians, a maxillary fenestra is present only in Kulindadromaeus, where it may be larger than the antorbital fenestra (Godefroit et al., 2014), and Hypsilophodon, where it is even smaller relative to the antorbital fenestra than in Haya (Galton, 1974a; Makovicky et al., 2011). IGM 100/3181 has a small prong dorsal to the buccal ridge that projects into the maxillary fenestra. It is matched by a less-pronounced knob on the interfenestral strut that projects ventrally into the maxillary fenestra (fig. 8). The interfenestral strut separating the maxillary and antorbital fenestra is also more strongly concave posteriorly at its midpoint in IGM 100/3181 (fig. 8) than in IGM $100 / 2017$ (fig. 3). This could be an artifact of slight crushing or displacement of the strut; however, the $83 \mathrm{~mm}$ long skull of IGM $100 / 3181$ is less dorsoventrally crushed overall than the $93 \mathrm{~mm}$ long skull of IGM 100/2017 (Makovicky et al., 2011; Norell and Barta, 2016). The antorbital fenestra is ovate, with the long axis of the oval directed anterodorsally, similar to the condition in Jeholosaurus (Barrett and Han, 2009). In comparison, the long axis of the antorbital fenestra is oriented anteroposteriorly in Thescelosaurus, Changchunsaurus, and Gasparinisaura (Coria and Salgado, 1996) and dorsoventrally in Hypsilophodon. The antorbital fenestra is 


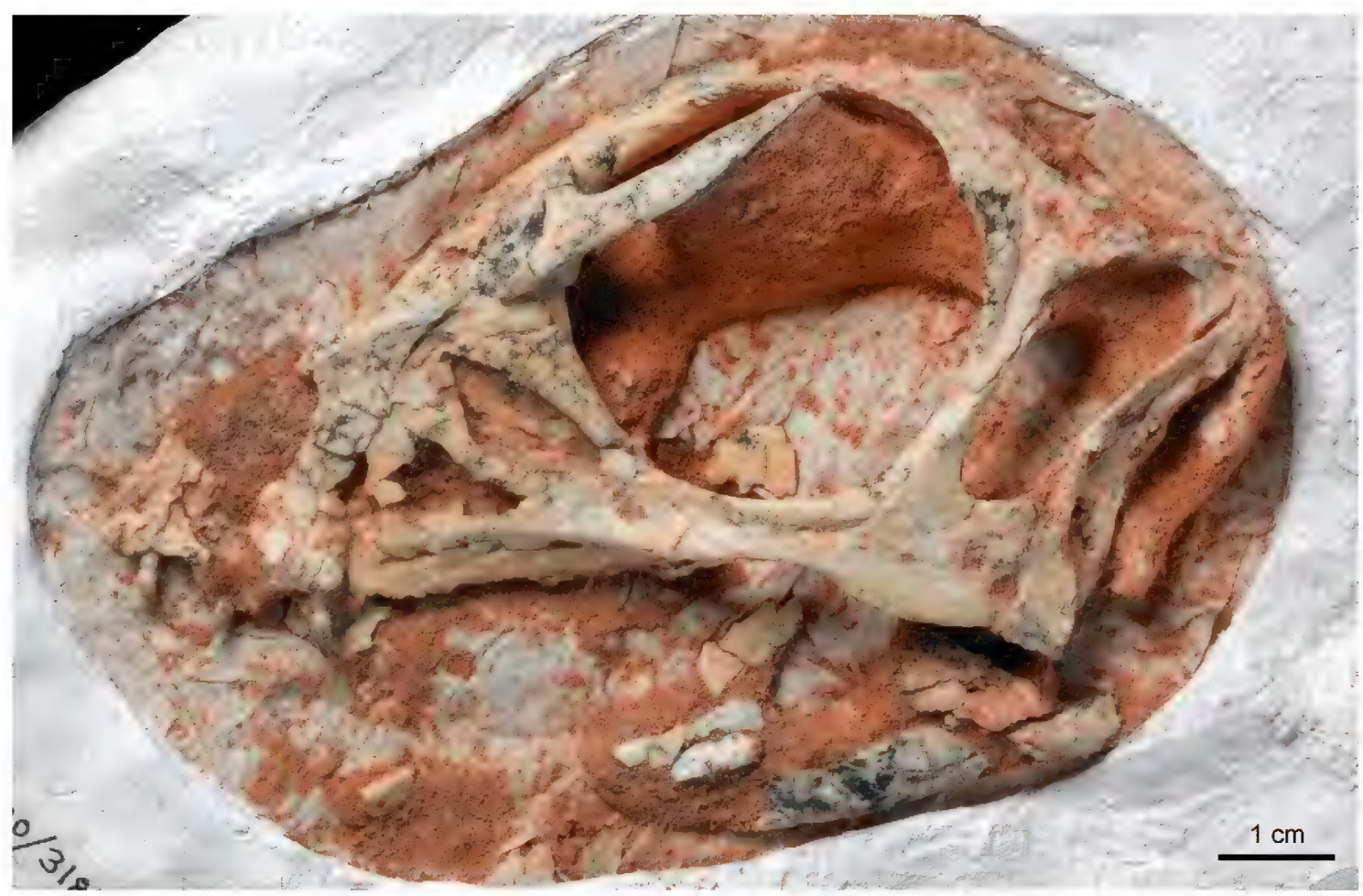

FIG. 8. Skull of Haya griva, IGM 100/3181, in left lateral view. Modified from Norell and Barta (2016).

nearly circular in Orodromeus (Scheetz, 1999), and more triangular in Agilisaurus and Hexinlusaurus (Barrett et al., 2005). A shallow antorbital fossa surrounds the fenestra and extends dorsally to the contact with the nasal and ventrally to meet the buccal ridge. A row of special foramina (Edmund, 1957) linked by a groove pierces the lingual surface of the maxilla. The special foramina of the dentary are described in more detail below.

As confirmed in CT slices, the right maxilla of IGM 100/2017 contains 14 alveoli, whereas the left contains 13. In the left maxilla, replacement teeth are present at the fourth, fifth, seventh, 10th, and 13th alveoli (counted from anterior to posterior). Replacement teeth in the fourth and 13th positions are partially erupted, with just the tips of the crowns protruding. The right maxilla contains replacement teeth at the first, fourth, fifth, seventh, eighth, 10th, 11th, and 14th alveoli (four of these are visible in the CT slice in figure
17A). Those at the fourth, seventh, and 10th tooth positions have partially erupted, whereas the others are deep within the maxilla. This suggests the presence of at least three "generations" of teeth in the right maxilla: (1) fully erupted, worn teeth (first, second, third, fifth, sixth, eighth, ninth, 11th, 12th, 13th, and 14th alveoli), (2) partially erupted, unworn teeth (fourth seventh, and 10th alveoli), and (3) unerupted teeth (first, fifth, eighth, 11th, and 14th alveoli). The right maxilla contains more replacement teeth than the left, even after accounting for the presence of an extra alveolus in the right maxilla. The degree of eruption of the replacement teeth also differs between right and left. This suggests that the timing of tooth replacement waves differed between the right and left maxillae, as may be the case for the right and left dentaries (see Dentary section below).

IGM 100/3178 (fig. 7) has 13 teeth in its left maxilla. The right is not preserved well enough 
to obtain a complete count. Unerupted replacement teeth are not clearly visible within the alveoli in CT slices for any of the tooth-bearing elements of IGM 100/3178 (there could be some that have erupted slightly, but this is difficult to discern in the CT slices).

Fourteen maxillary teeth in Haya griva is within the range of most other basal neornithischians, which may display one or two teeth greater or fewer than found in Haya griva. This is with the exception of the greatly enlarged maxillary tooth counts (18-30) of Hexinlusaurus, Kulindadromaeus, Isaberrysaura, and Thescelosaurus (Barrett et al., 2005; Boyd, 2014; Godefroit et al., 2014; Salgado et al., 2017). The number of maxillary tooth positions increases through ontogeny in Orodromeus and possibly in Kulindadromaeus (Godefroit et al., 2014). This is also likely true of Haya, given the short length of the maxilla in the specimen with juvenile morphology (IGM 100/2016); however, maxillary alveoli are not well preserved in this specimen. The teeth are low crowned and triangular, with at least 10 denticles distributed evenly between the mesial and distal carinae on unworn teeth in the right maxilla of IGM 100/2017. The teeth exhibit lingual wear facets. The crowns of unworn and worn teeth appear somewhat asymmetric in buccal view, with the apical edge sloping basodistally, as in Changchunsaurus and Hypsilophodon (Galton, 1974a; Jin et al., 2010) (fig. 17B). This asymmetry stands in contrast to the more symmetrical maxillary teeth of heterodontosaurids and Lesothosaurus (Sereno, 1991, 2012). The maxillary teeth of Thescelosaurus change from symmetrical anteriorly being more asymmetrical posteriorly (Boyd, 2014). In Haya there is no clear change in the degree of asymmetry along the toothrow. The asymmetric maxillary teeth of Haya also differ from its symmetrical dentary teeth, as is the case for Hypsilophodon (Galton, 2007).

LACRIMAL: The straplike lacrimal is gently concave along its anterior and posterior borders, anterodorsally oriented, and contacts the dorsal process and posterodorsal corner of the maxilla (figs. 3, 5-8). It also contacts the prefrontal and anterior supraorbital dorsally, and the jugal posteroventrally. The lacrimal of Haya is relatively narrower and more elongate than the more triangular lacrimal of Thescelosaurus, and is straighter than the lacrimals of Agilisaurus (Peng, 1992; Barrett et al., 2005), Kulindadromaeus (Godefroit et al., 2014), Hypsilophodon (Galton, 1974a), and a probable juvenile Jeholosaurus (IVPP V12530), which all form inverted L-shapes (Barrett and Han, 2009). The dorsal end of the lacrimal flares slightly anteriorly in at least one Haya specimen (IGM 100/3181), similar to the lacrimals of Lesothosaurus and Changchunsaurus (Jin et al., 2010; Porro et al., 2015). The lacrimal of IGM 100/3181 may have contacted the subnarial process of the premaxilla along a very small margin (fig. 8), in contrast to IGM $100 / 2017$, in which these two bones do not meet (Makovicky et al., 2011) (fig. 3). The premaxilla and lacrimal develop an extensive contact through ontogeny in Jeholosaurus (Barrett and Han, 2009). The two bones contact each other in Kulindadromaeus (Godefroit et al., 2014), and a still more extensive contact is present among iguanodontians (Norman, 2004). The premaxilla and lacrimal do not articulate in other earlydiverging neornithischians nor Hypsilophodon (Norman et al., 2004). Posteriorly, the lacrimal contains a dorsoventrally oriented groove, possibly contiguous with the lacrimal foramen. In IGM 100/2019, the right lacrimal contains a small depression (possibly the lacrimal foramen), the lateral border of which forms a pronounced ridge that curves slightly medially toward the dorsal end of the lacrimal.

NASAL: The nasals are roughly triangular in dorsal view, contacting the subnarial processes of the premaxillae anterolaterally, the prefrontals posterolaterally, and the frontals posteriorly (fig. 4A, C). Unlike Thescelosaurus (Boyd, 2014), but similar to most early-diverging neornithischians, the nasal of Haya does not contribute to the border of the antorbital fenestra. The nasals are mediolaterally broadest above the anteroposterior midpoint of the maxillae and taper posteri- 


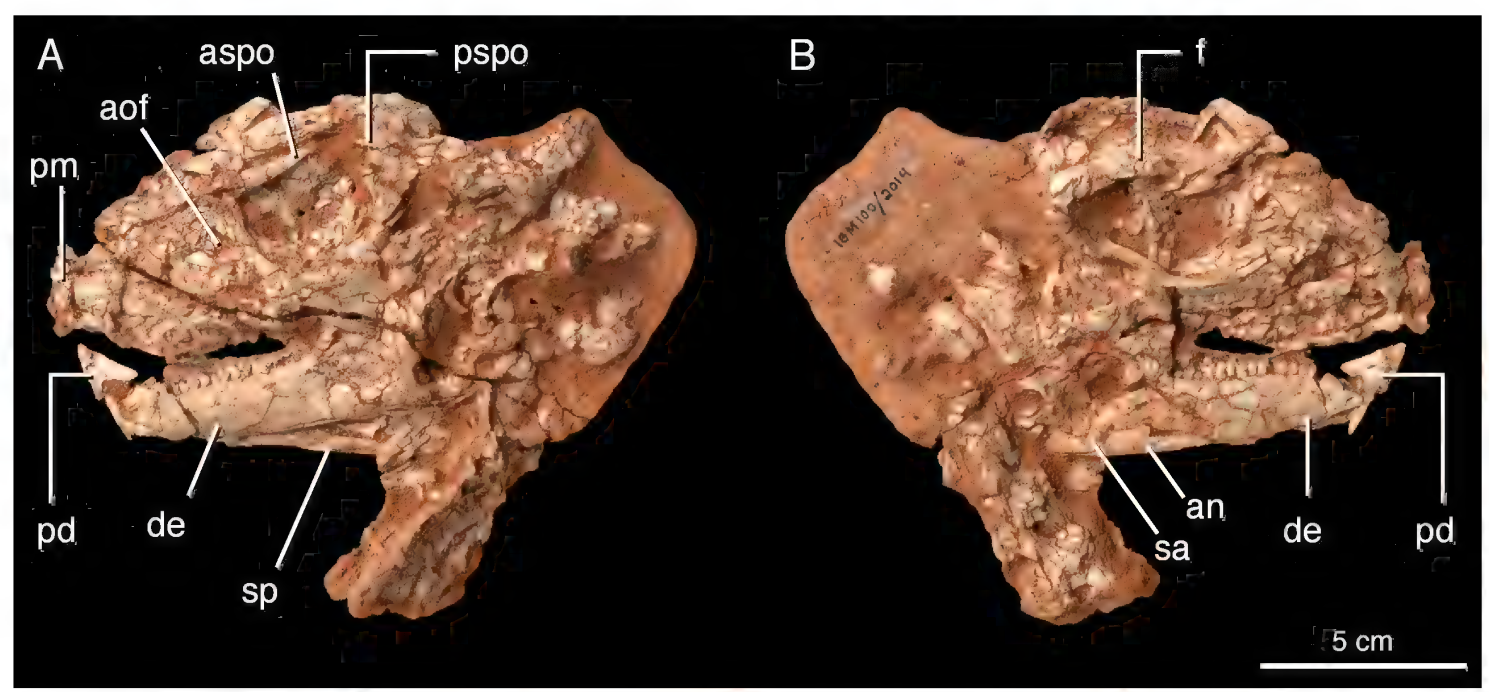

FIG. 9. Skull and cervical vertebrae of Haya griva, IGM 100/2014, in A, left lateral and B, right lateral views. Abbreviations in appendix 1.

orly near their contact with the frontals. The posterior end flares somewhat laterally, forming a ridge along the contacts with the maxilla and prefrontal that borders the antorbital fossa dorsally. In Haya, the nasals are not as extensively exposed in lateral view as those of some other early-diverging neornithischians (e.g., Isaberrysaura, Jeholosaurus, Thescelosaurus), though the nasal region is not often preserved among taxa of this grade (Norman et al., 2004). The nasals are unfused along the internasal suture. They dip toward one another, forming a shallow midline depression along the length of their suture (fig. 4A), as in Heterodontosaurus, Agilisaurus, Hexinlusaurus, Kulindadromaeus, Jeholosaurus, and Changchunsaurus (He and Cai, 1984; Peng, 1992; Barrett and Han, 2009; Jin et al., 2010; Makovicky et al., 2011; Norman et al., 2011; Godefroit et al., 2014; Norell and Barta, 2016). This depression deepens anteriorly. The nasals are mostly flat except near the midline depression and at their anterior ends, which arch above the naris. The anterior ends of the frontals wedge between the posterior ends of the nasals (Makovicky et al., 2011). The prefrontals overlap the posterior half of the nasals along an extensive flat contact. A single nutrient foramen is present near the broadest portion of each nasal (Makovicky et al., 2011; fig. 4A). Multiple such foramina are present in Jeholosaurus and Thescelosaurus (Barrett and Han, 2009; Boyd, 2014). As noted by Makovicky et al. (2011), the internarial bar is not preserved, but the anterior ends of the nasals leave little space for the premaxillae to wedge between them, in contrast to the nasal-premaxilla articulation of Hypsilophodon (Galton, 1974a).

JUGAL: The jugal is mediolaterally compressed and forms the ventral borders of the orbit and lateral temporal fenestra (figs. 3, 5-8, 10). The anterior (maxillary) ramus is dorsoventrally narrower than the posterior (quadratojugal) ramus. Overall, the jugals of Haya and most other earlydiverging neornithischians are dorsoventrally narrow and anteroposteriorly long, compared to the dorsoventrally wider jugal of Hypsilophodon (Galton, 1974a). The jugal thickens mediolaterally at the level of the dorsal (postorbital) process. The postorbital overlaps the dorsal process of the jugal dorsally along an anterolaterally facing scarf joint. The dorsal process of IGM $100 / 2017$ is more posteriorly directed than in other specimens, but this likely is a consequence of the slight crushing and shearing of the holo- 
type skull. In IGM 100/3181, the dorsal process exhibits a well-defined dorsoventrally directed ridge on its lateral surface (fig. 8). Unique to Haya and Heterodontosaurus among noncerapodan ornithischians is a bifid anterior process of the jugal (figs. 3, 6, 8). In IGM 100/2017 and IGM 100/2019, the dorsal prong of this bifurcation appears to reach the lacrimal (as in Heterodontosaurus), and the ventral prong articulates solely with the jugal process of the maxilla. It is difficult to determine whether the dorsal prong would have reached the lacrimal in IGM 100/3181 because of poor preservation. In Heterodontosaurus the anterior jugal bifurcation forms the entrance to a deep lateral channel connecting to the antorbital fossa (Norman et al., 2011; Sereno, 2012). Haya lacks such a deep channel; instead, the lateral surface of the anterior process contains a shallower suborbital sulcus in all specimens. In IGM 100/3181 and IGM 100/2016 this sulcus houses at least five small foramina that are not present in other Haya specimens or other taxa (Norell and Barta, 2016). The slight degree of curvature of the anterior process is similar to that of Thescelosaurus (Boyd, 2014) and Changchunsaurus (Jin et al., 2010). It lacks the extreme dorsal curvature seen in Agilisaurus (Peng, 1992; Barrett et al., 2005). The anterior process does not taper as sharply as in Hexinlusaurus, Agilisaurus, and Jeholosaurus. Instead, the dorsal and ventral borders of the process are largely parallel to one another for their entire lengths. Posteriorly, the jugal is bifid, with both prongs overlapping the quadratojugal. IGM 100/2016 best illustrates the bifid nature of the jugal (Makovicky et al., 2011), with two nearly equal-length prongs forming a $\mathrm{V}$-shape where they overlap the quadratojugal (fig. 10). As noted by Makovicky et al. (2011), the appearance of this V-shape may be exaggerated by bone loss between the prongs. However, the angle between the prongs is a much shallower U-shape in many other specimens (IGM 100/2017, IGM 100/2019, IGM $100 / 3178$ and IGM 100/3181), and it seems likely that this shape difference in jugal prongs compared to IGM $100 / 2016$ is at least partially bio- logic. The prongs are roughly equal in size in IGM 100/3181 (fig. 8), whereas the dorsal prong is narrower and more pointed than the ventral prong in IGM 100/2017, IGM 100/3178, and IGM 100/2019 (figs. 3, 6, 7). The prongs both overlap the quadratojugal, in contrast to Thescelosaurus, Tenontosaurus, and Zalmoxes, where the dorsal prong overlaps the lateral surface of the quadratojugal and the ventral prong the medial surface (Weishampel et al., 2003; Makovicky et al., 2011; Boyd, 2014). The surface of the jugal in all examined Haya specimens is smooth, with no trace of the jugal rugosities seen in some Jeholosaurus and Changchunsaurus specimens (Barrett and Han, 2009; Jin et al., 2010). It further lacks the jugal bosses present in Heterodontosaurus, Manidens, Zephyrosaurus, and Orodromeus (Sues, 1980; Scheetz, 1999; Norman et al., 2011; Pol et al., 2011).

QUADRATOJUGal: The quadratojugal is a small, flat element situated between the jugal laterally and the quadrate medially (figs. 3, 6-8, 10). It is trapezoidal in overall shape, with a dorsal border that is shorter than its ventral border (Makovicky et al., 2011), whereas the exposed portion of the quadratojugal is more triangular in Orodromeus and somewhat teardrop shaped in Zalmoxes and some Dryomorpha (Scheetz, 1999; Norman, 2004). It has a pointed dorsal extension as in Agilisaurus, Thescelosaurus, and many other early-diverging neornithischians (Norman et al., 2004; Barrett et al., 2005; Boyd, 2014). The quadratojugal is relatively dorsoventrally tall and contributes more extensively to the posterior border of the lateral temporal fenestra than to the ventral border. This dorsal process of the quadratojugal is splintlike and appressed to the anterior margin of the quadrate (fig. 6). Its dorsalmost point reaches the anteriormost projection of the quadrate in lateral view as in Agilisaurus, Jeholosaurus, Changchunsaurus, Thescelosaurus, and Hypsilophodon (Galton, 1974a; Peng, 1992; Barrett and Han, 2009; Jin et al., 2010; Boyd, 2014). This process does not reach the squamosal, unlike in Heterodontosaurus and possibly Lesothosaurus and Gasparin- 


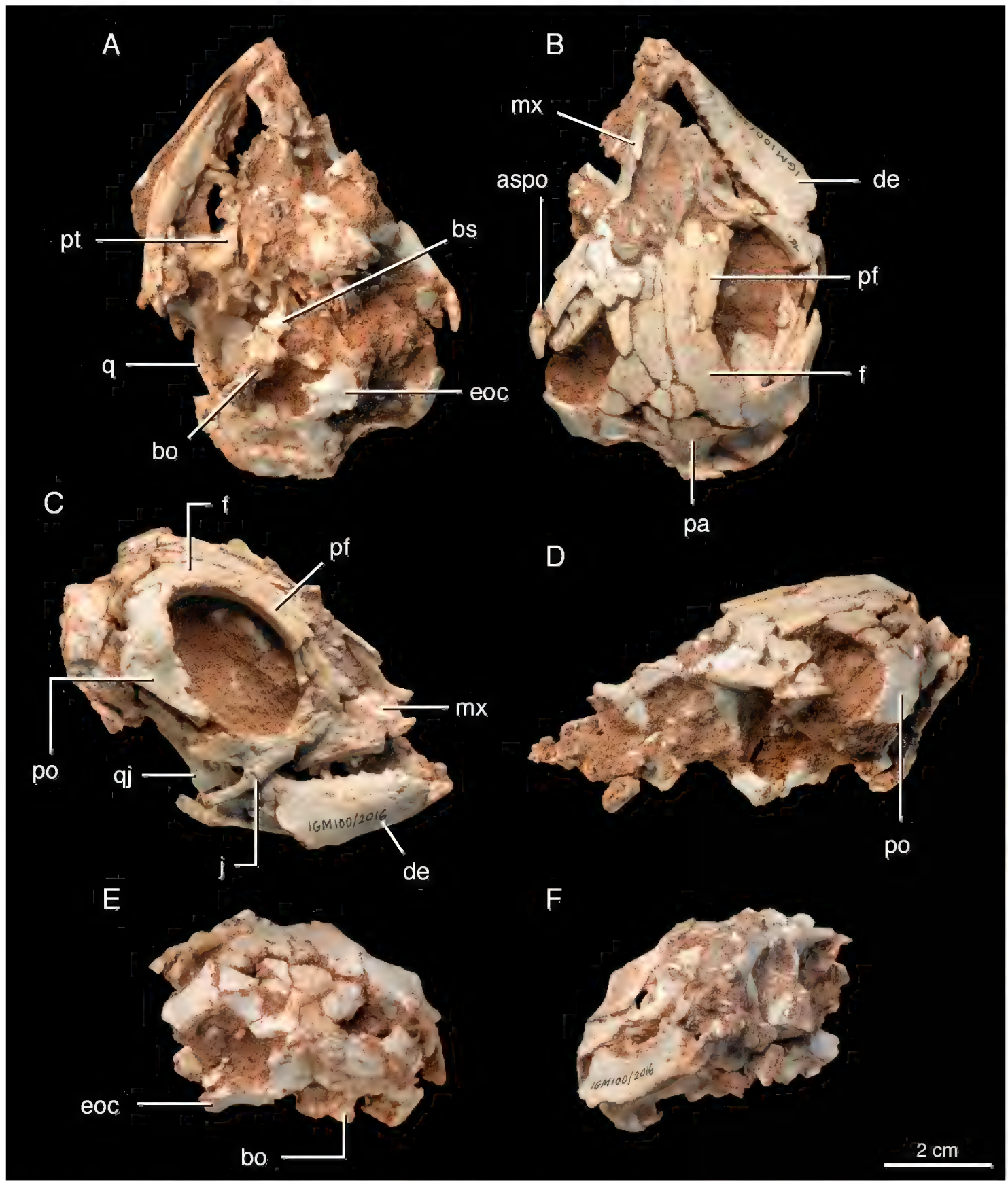

FIG. 10. Skull of Haya griva, IGM 100/2016 in A, ventral, B, dorsal, C, right lateral, D, left lateral, E, posterior, and $\mathbf{F}$, anterior views. Abbreviations in appendix 1. 
isaura (Coria and Salgado, 1996; Norman et al., 2011; Porro et al., 2015). The quadratojugal of Haya lacks any significant anterior process, in contrast to the L-shaped quadratojugals of Parksosaurus and especially Gasparinisaura (Galton, 1973; Coria and Salgado, 1996). In lateral view, it overlaps and obscures most of the quadratojugal wing of the quadrate. Dorsally, the quadratojugal contributes to the posteroventral border of the lateral temporal fenestra. Ventrally, the border of the quadratojugal is straight and parallels the anteroposterior axis of the skull. The posteroventral corner projects ventrally to the same level as the ventral edge of the jugal in lateral view. It lacks the extremely long posteroventral process of heterodontosaurids (Norman et al., 2011; Pol et al., 2011; Sereno, 2012), and the lesser posteroventral expansions present in Changchunsaurus, Parksosaurus, and Thescelosaurus (Galton, 1973; Jin et al., 2010; Boyd, 2014). Centrally, a large quadratojugal foramen pierces the quadratojugal, as in Hypsilophodon and Jeholosaurus (Galton, 1974a; Barrett and Han, 2009). The long axis of this ovate foramen is oriented anteroposteriorly. Much smaller foramina are present in the quadratojugals of Thescelosaurus and Gasparinisaura (Coria and Salgado, 1996; Boyd, 2014). There may be a small notch present in the posteroventral corner of the quadratojugals of IGM 100/2017 and IGM 100/2016 (figs. 3, 10). In IGM $100 / 3181$, the quadratojugal foramen appears to pierce the suture between the jugal and quadratojugal (Norell and Barta, 2016; fig. 8), somewhat similar to the condition in Tenontosaurus (Norman, 2004); however, this could be an artifact of displacement or erosion in this specimen.

Postorbital: As in most basal neornithischians, the postorbital is a triradiate bone, with processes that contact the frontal, parietal, jugal, and squamosal (figs. 3, 6-10). There does not appear to be any contact with the laterosphenoid, as occurs in Hypsilophodon (Galton, 1974a). The anteroventrally directed ventral process extensively overlaps the dorsal (postorbital) process of the jugal along a scarf joint to form the posterior border of the orbit. The posterior process articu- lates with the squamosal, with the posterior process forming a simple triangular prong on IGM 100/3178 (fig. 7) and a possibly bifid prong on IGM 100/2017 and IGM 100/2019 (figs. 3, 6). The anterior process contacts the frontal and parietal near where those two bones meet. The anterior process of Haya is straight and straplike, being less anteroposteriorly expanded than the postorbital of Orodromeus (Scheetz, 1999). A deep sulcus extends dorsoventrally across the lateral side of the postorbital in IGM 100/3181 (fig. 8), but it is less evident in the other Haya specimens, being nearly absent or forming a shallow, U-shaped trough (IGM 100/2016). This differs from the more extensive postorbital fossa in Heterodontosaurus (Sereno, 2012). Viewed anteriorly, the orbital margin of the ventral process possesses a shallow dorsoventrally oriented trough. The posterior edge of the postorbital contributes to the lateral border of the supratemporal fenestra. The right postorbital of IGM 100/2016 has a somewhat pronounced and laterally directed protuberance (the "postorbital process" of Makovicky et al., 2011) that projects anteriorly into the orbit (fig. 10). This feature is not strongly developed in IGM 100/3181 and IGM 100/3178 (figs. 7, 8), but is present (though not as laterally directed) in IGM 100/2014 and IGM 100/2017 (figs. 3, 9). Orodromeus and Zephyrosaurus possess similar protuberances (Sues, 1980; Scheetz, 1999). As revealed by the intact posterior supraorbitals of IGM 100/2019 and IGM 100/3178 (figs. 6, 7), this rugose protuberance is indeed the attachment site for the posterior supraorbital, as hypothesized by Norman et al. (2004) and Makovicky et al. (2011).

QUADRATE: Overall, the quadrate is fairly morphologically conservative among earlydiverging neornithischians and closely related taxa (Norman et al., 2004). It is bowed and narrow in lateral view, but broadens into a medial flange, the pterygoid wing, that articulates along a broad, flat contact with the pterygoid (figs. 4B, $10 \mathrm{~A})$. Another flange, the jugal wing, flares laterally above its contact with the quadratojugal. The two diverging wings give the quadrate a keeled 
appearance in posterior view (with sulci on either side of the posterior ridge), and, inversely, a trough in anterior view. Dorsally, a rounded head articulates with the corresponding cotylus of the squamosal. This head is not as deflected from the shaft as it is in Hypsilophodon, Zephyrosaurus, and Dryosaurus (Galton, 1974a, 1983; Sues, 1980; Makovicky et al., 2011), but it is more recurved than the more columnar quadrate of Agilisaurus (Peng, 1992). Articulation with the squamosal almost completely prevents the head of the quadrate from contacting the paraoccipital process of the exoccipital as in Thescelosaurus (Boyd, 2014; fig. 6). The right quadrates of IGM 100/2014 and IGM 100/2016 (figs. 9, 10) may be less bowed than those of IGM 100/2017 and IGM 100/2019 (figs. 3, 6); however, the quadrate of IGM $100 / 2014$ is broken and separated at midshaft, complicating assessment of its overall shape. Ventrally, the two rounded condyles of the distal end contact the articular. There is little separation between these two condyles in IGM 100/2019 (fig. 18B, 19). The lateral condyle projects laterally beyond the mandible. The quadrate forms most of the posterior border of the lateral temporal fenestra, in contrast to Gasparinisaura and some basal iguanodontians, where the quadrate is partially or completely excluded from this fenestra by the squamosal and jugal or quadratojugal (Coria and Salgado, 1996; Norman, 2004). The right quadrate of IGM 100/2016 bears deeper depressions on both its pterygoid and jugal wings than IGM 100/2017. These depressions are offset from one another, with the medial one placed more dorsally on the bone, and the lateral one more ventrally. The quadratojugal is tightly appressed to the quadrate in Haya; therefore, it is difficult to assess whether or not a paraquadrate foramen is present, as in Heterodontosaurus and some iguanodontians (Norman, 2004). A depression between the two bones in IGM 100/2017 (fig. 3), pockmarks on the lateral surface of the quadrate in IGM 100/3181 (Norell and Barta, 2016), and a damaged hole between the quadratojugal and quadrate in IGM 100/2019 (figs. 6, 19) suggest the presence of a small paraquadrate or quadrate foramen. However, the poor quality of these specimens prevents further assessment.

Squamosal: The squamosal forms a bar that contributes to most of the dorsal border of the lateral temporal fenestra and the lateral border of the supratemporal fenestra (figs. 3, 4, 6-8). It contacts the postorbital anteriorly, the quadrate posteroventrally, the parietal medially, and the exoccipital posteriorly. An asymmetrical $\mathrm{V}$-shaped suture joins the postorbital and squamosal. This suture is more symmetric in IGM 100/3178 (fig. 7) than in IGM 100/2017 (figs. 3, 4 ), and is more clearly S-shaped in IGM 100/2019 (fig. 6), forming an interlocking contact between the two bones. In all Haya specimens, this contact is less extensive than the long tongue-ingroove suture of Thescelosaurus neglectus, being more similar to the simpler suture of T. assiniboiensis (Brown et al., 2011: fig 6). However, the offcenter lateral overlap of the two bones in at least IGM 100/2017 (fig. 4A) is distinct from the more symmetric, dorsally oriented sutural surface of $T$. assiniboiensis. The prequadratic process of the squamosal fits into the groove on the anterior surface of the quadrate, while a short, broad flange folds ventrally under the posterior surface of the quadrate head to form a cotyle for the head of the quadrate. This posterior flange is itself overlain by the exoccipital (fig. 6). Posteriorly, a short, slightly medially directed knob arises from the body of the squamosal to contact a dorsal process of the exoccipital. Medial to its articulation with the quadrate, the squamosal is strongly concave anteriorly in dorsal view where it contributes to the posterior border of the supratemporal fenestra. The right squamosal of IGM 100/2016 (fig. 10) is less strongly concave anteriorly than in IGM 100/2017 (figs. 3, 4A, C). A deep triangular fossa, contiguous with the lateral temporal fenestra and defined primarily by the overhang of the dorsal portion of the squamosal roofing the prequadratic process, excavates the lateral surface of the squamosal. According to (Ostrom, 1961) and Galton (1974), this fossa likely contained the origin for $\mathrm{M}$. 
adductor mandibulae externus superficialis, generally consistent with its origin in extant lepidosaurs, crocodylians, and birds (Holliday, 2009). This fossa is not as well defined in IGM 100/2016 as it is in IGM 100/2017.

PARIETAL: In dorsal view, the parietals are broad, flat, dorsolaterally facing bones meeting along a midline sagittal crest (figs. 4A, 10B); a feature typically present in neornithischians, though it is weak to absent in Lesothosaurus (Knoll, 2002a, 2002b; Porro et al., 2015). The parietals are fused in IGM 100/2017 (fig. 4A) and at least partially fused posteriorly in IGM 100/2016 (fig. 10B). The sagittal crest bifurcates anteriorly (Makovicky et al., 2011). The suture with the frontals is extensive and V-shaped, with the parietals accommodating the posterior processes of the frontals, as in Jeholosaurus and Thescelosaurus (Barrett and Han, 2009; Boyd, 2014). Haya lacks a median process inserting between the frontals, a feature exposed on the dorsal surface of the skull in Hypsilophodon and Dryosaurus (Galton, 1974a, 1983), and ventrally in Thescelosaurus (Boyd, 2014). The posterior thirds of the parietals laterally flare and are divergent, forming the nuchal crest, which defines part of the posterior borders of the supratemporal fenestrae. In posterior view, the wings of the parietal laterally surround the supraoccipital (fig. 18). These wings are mediolaterally wider and more dorsally arched in posterior view than those of Lesothosaurus (Porro et al., 2015), Thescelosaurus (Boyd, 2014), Hypsilophodon (Galton, 1974a), and iguanodontians (Norman, 2004). The parietal has an anteroposteriorly short contact with the squamosal, as in Jeholosaurus and Hypsilophodon (Galton, 1974a; Barrett and Han, 2009), in contrast to the more extensive contact of some iguanodontians and marginocephalians (Maryanska et al., 2004; Norman, 2004; You and Dodson, 2004). Ventrally, the parietals form part of the dorsal roof of the endocranial space. Laterally, the postorbital overlaps the parietal. The parietal projects a short process to contact the base of the postorbital bar (Makovicky et al., 2011). The parietals of IGM 100/2016 (fig. 10B) are anteroposteriorly shorter than those of IGM 100/2017 (fig. 4A, C) and bear a more anteriorly directed nuchal crest. A short sagittal crest is present in IGM 100/2016, but whether it anteriorly bifurcates is not apparent. The lateral processes of the parietal are short and apparently do not contribute to the base of the postorbital bar.

FrONTAL: The frontal is an anteroposteriorly long, relatively dorsoventrally thin, rectangular bone that meets the nasal anteriorly, the parietal posteriorly, and the postorbital posterolaterally (figs. 3, 4A, C, 8-10). The frontal forms most of the dorsal margin of the orbit. It is narrowest at its midpoint over the orbit. The frontal thickens slightly in dorsoventral height from its anterior to posterior ends. The anterior processes of the two frontals articulate between the posterior processes of the nasals along a $\mathrm{V}$-shaped suture. The frontal contains a deep, narrow groove that receives the pointed posterior end of the prefrontal laterally. This groove curves ventrally, in contrast to the dorsally curving groove in Tenontosaurus (Thomas, 2015). The suture with the parietal is relatively mediolaterally straight (Makovicky et al., 2011). As in many other ornithischian taxa (but not Lesothosaurus; Porro et al., 2015), the orbital margin of the frontal bears minute striations that create a rugose edge (Norell and Barta, 2016; fig. 8), likely representing an attachment site for connective tissue that stretched to the anterior supraorbital, laterally (Maidment and Porro, 2010). The frontal is arched along its length in lateral view and faces anterodorsally in uncrushed specimens, such as IGM 100/3181 (Norell and Barta, 2016; fig. 8). IGM 100/3181, IGM 100/3178, and IGM 100/2019 (figs. 6-8) likely provide a better overall idea of the form and dimensions of the skull roof bones in lateral view, as they are not as dorsoventrally compressed as in the holotype, IGM $100 / 2017$ (fig. 3). The maximum length to width ratio of the frontal is 2.9 in IGM 100/2017, nearly identical to the ratio in Jeholosaurus, Agilisaurus, Hypsilophodon, and Zephyrosaurus, but greater than that of Orodromeus, Hexinlusaurus, Thescelosaurus, and basal ceratopsians (Barrett and 


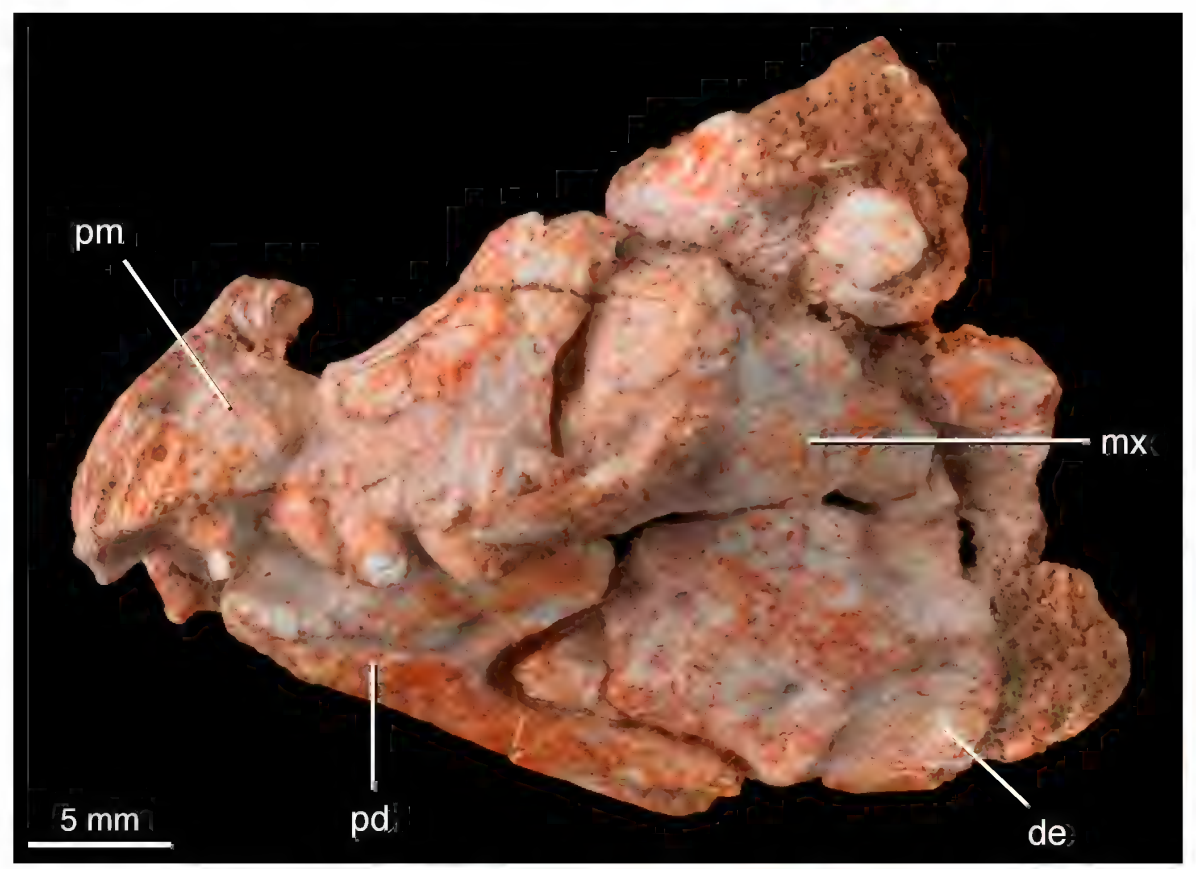

FIG. 11. Premaxilla, predentary, partial maxilla, and partial dentary of Haya griva, IGM 100/2018. Abbreviations in appendix 1 .

Han, 2009). Makovicky et al. (2011) described a significant narrowing of Haya frontals through ontogeny. This is not supported by our revised measurements, which found a maximum frontal length:width ratio in IGM 100/2016 of 2.8, a ratio in IGM 100/2017 of 2.9, and a ratio in IGM $100 / 3567$ of approximately 2.5 . Makovicky et al. (2011) likely used too narrow a width measurement for the frontals of IGM 100/2017, which produced the discrepancy between their findings and the current work. The left frontal of IGM 100/3181 (fig. 8) appears relatively longer and narrower than that of IGM 100/2017 (figs. 3, 4A, C). In a possible ontogenetic difference, the frontals of IGM 100/2016 contact the parietals further posteriorly on the skull than in IGM $100 / 2017$, a consequence of the overall smaller size and ventral deflection of the parietal and occipital region in IGM 100/2016. The suture between the frontals appears unfused along its length in IGM 100/2016 (fig. 10B). In IGM $100 / 2017$, the suture between the two bones appears as a line of higher density than the matrix (and of similar density to the bones) in the CT scans, though this is frequently destroyed by postmortem cracks that follow the suture. Given that this suture is still visible in places, it is clear that the frontals were not completely fused in IGM 100/2017. The length of the prefrontal suture is also relatively longer in IGM $100 / 2016$ than in IGM 100/2017.

An isolated near-complete right frontal (IGM 100/3567), somewhat smaller than the corresponding element in the holotype, reveals additional details not readily observed in articulated specimens. The ventral surface of the frontal possesses a prominent ventral ridge, the crisa cranii, inset medially from the orbit and running anteroposteriorly, as in Lesothosaurus (Porro et al., 2015). Anterior and medial to this ridge is the posterior end of the olfactory tract and posterior to the ridge lies a small portion of the cerebral fossa (Galton, 1989). The concave ventral surface between the orbital margin and ventral ridge is mediolaterally narrower in Haya than in Dryosaurus and Tenontosaurus (Galton, 1989; 
Thomas, 2015). The frontals interlock with one another via anterodorsally directed ridges and grooves on the median sutural surface. The articular facet for the postorbital is triangular, and is directed slightly ventrally, as in Zephyrosaurus and Thescelosaurus (Sues, 1980; Boyd, 2014).

Prefrontal: The prefrontal is a bladeshaped, dorsally convex bone that overlaps slightly less than half of the length of the frontal, fitting into a narrow groove on its dorsal surface (figs. 4A, C, 8, 10, 12). The prefrontal is broadest and thickest anterolaterally and tapers and thins posteriorly. The prefrontals of IGM 100/2016 (fig. 10) are longer relative to the frontals than those of the other specimens. The nasal contacts the prefrontal medially for much of its length in IGM 100/2017 (fig. 4A, C). This contact is less extensive in IGM 100/3181, with the medial contact with the frontal more extensive in this specimen. The anterior process of the prefrontal abuts the maxilla, but not as extensively as in Parksosaurus (Galton, 1973; Boyd, 2014). The anterior supraorbital articulates with the prefrontal along much of its lateral and posterolateral surfaces. The lacrimal overlaps a short ventral process. The morphology of this ventral process is difficult to assess in articulated specimens. In IGM $100 / 3181$ (fig. 8) the ventral process of the prefrontal appears to be relatively short, as in Thescelosaurus (Boyd, 2014), in contrast to the extremely long ventral process of Tenontosaurus (Thomas, 2015). The prefrontal contributes to the anterodorsal margin of the orbit. The left prefrontal of IGM 100/3181 (fig. 8) is relatively narrower and more pointed at its posterior end than that of IGM 100/2017 (fig. 4A, C). In IGM 100/3181, possibly IGM 100/3557, and IGM $100 / 2016$ (figs. $8,10,12$ ), a small foramen pierces the dorsal surface of the bone about one third the distance from its anterior margin, at the level of the articulation with the anterior supraorbital (Norell and Barta, 2016). This foramen also is observed in Thescelosaurus and previously was considered autapomorphic for that taxon (Boyd et al., 2009; Boyd, 2014). A prefrontal foramen may be present in IGM 100/2016, but its depth and extent are concealed by matrix.

Supraorbitals: Though the bony elements overhanging the orbit in ornithischian dinosaurs have often been referred to as "palpebrals" (e.g., Norman et al., 2004; Makovicky et al., 2011; Norell and Barta, 2016), other authors (Maidment and Porro, 2010; Nesbitt et al. 2012) recommend against using this term based on the nonhomology of this element with the crocodilian palpebral. We follow their suggestions and here refer to these bones as the anterior and posterior supraorbitals sensu Salgado et al. (2017). These are equivalent to the supraorbital and accessory supraorbital, respectively, of Boyd (2014). The anterior supraorbital is triradiate, with a recurved dorsal process that contacts the prefrontal, an anterior process that contacts the prefrontal-lacrimal junction, and a posterior process that extends across the orbit (figs. 3, 4A, C, 6-10, 12). A small ventral process abuts the lacrimal. The anterior supraorbital is thickest anteriorly and thins posteriorly. In lateral view, it is dorsally concave, in contrast to Heterodontosaurus, Jeholosaurus and Thescelosaurus, in which it is dorsally convex (Barrett and Han, 2009; Norman et al., 2011; Boyd, 2014). Laterally, a well-defined ridge runs the length of the anterior supraorbital. The dorsal surface of the anterior supraorbital is striated, probably for connective tissue attachment with the corresponding rugose surface of the frontal (see above). The left anterior supraorbital of IGM $100 / 3178$ has a smoother dorsal surface than that of IGM 100/2017. The left anterior supraorbital of IGM $100 / 3181$ (fig. 8) is directed somewhat more dorsally than those of the other specimens, but this is likely because IGM $100 / 3181$ is less dorsoventrally crushed than they are. The posterior end of the anterior supraorbital extends nearly to the level of the dorsal margin of the orbit in lateral view. The anterior supraorbital appears to lengthen relative to the orbit during ontogeny in Orodromeus (Scheetz, 1999); however, this cannot yet be assessed for Haya. 


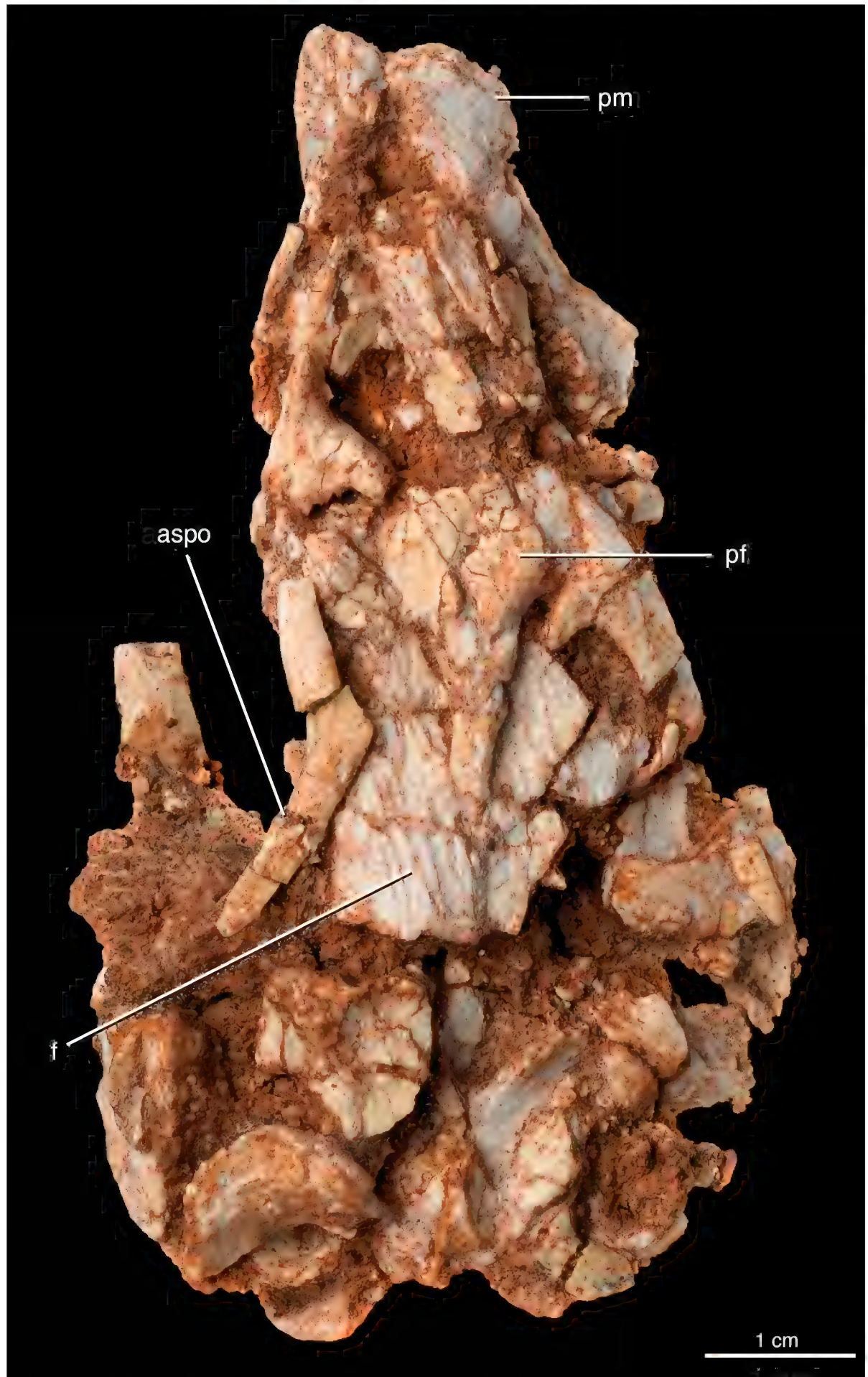

FIG. 12. Skull of Haya griva, IGM 100/3557 in dorsal view. Abbreviations in appendix 1. 


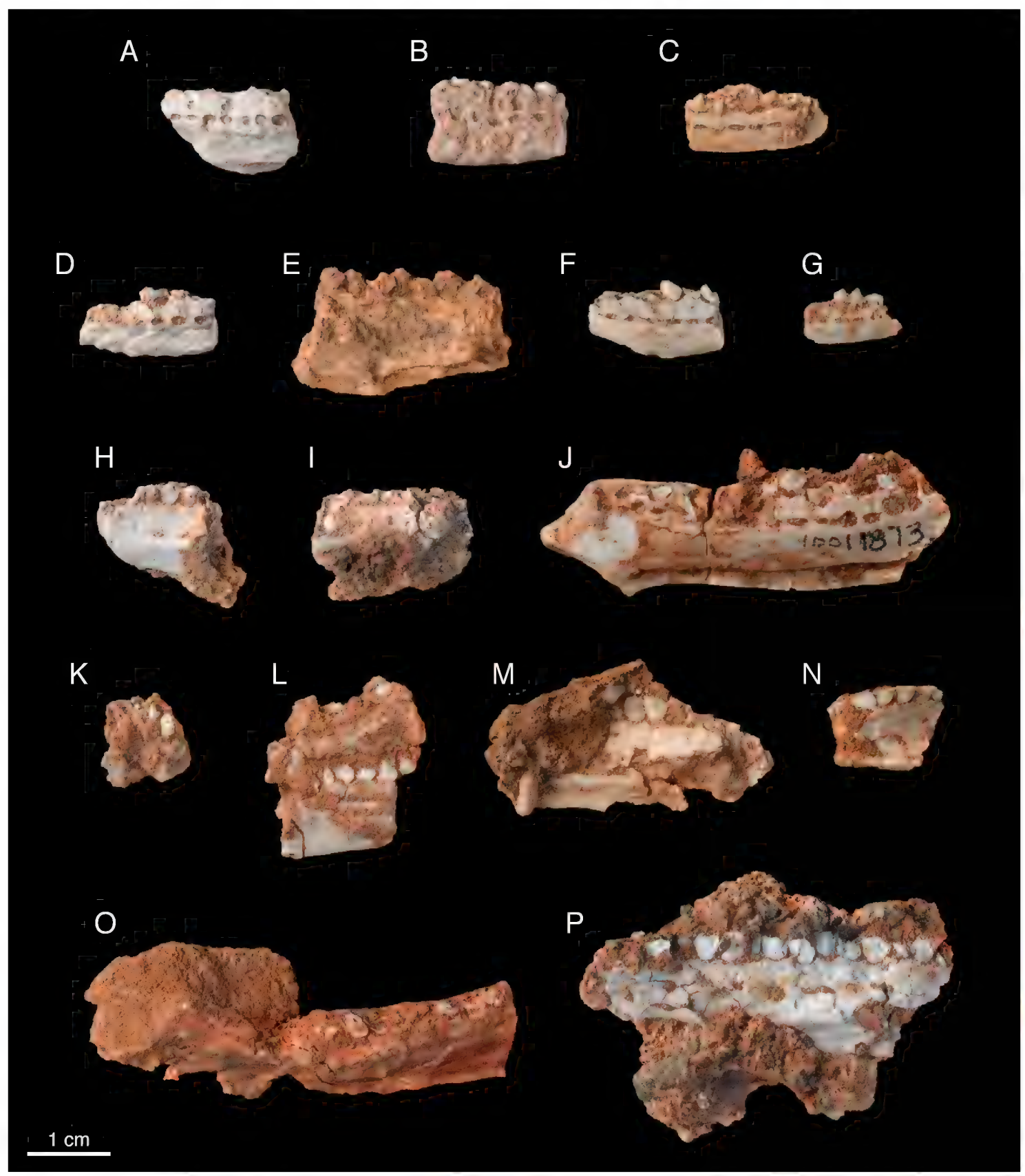

FIG. 13. Maxilla and dentary fragments of Haya griva. A, IGM 100/3568, B, IGM 100/3562, C, IGM 100/3569, D, IGM 100/3566, E, IGM 100/3604, F, IGM 100/3564, G, IGM 100/3564, H, I, IGM 100/3565 (two pieces), J, IGM 100/1873, K, MAE 06-6, L, IGM 100/3559, M, IGM 100/3563, N, MAE 06-64, O, IGM 100/1322, P, MAE 03-21. 


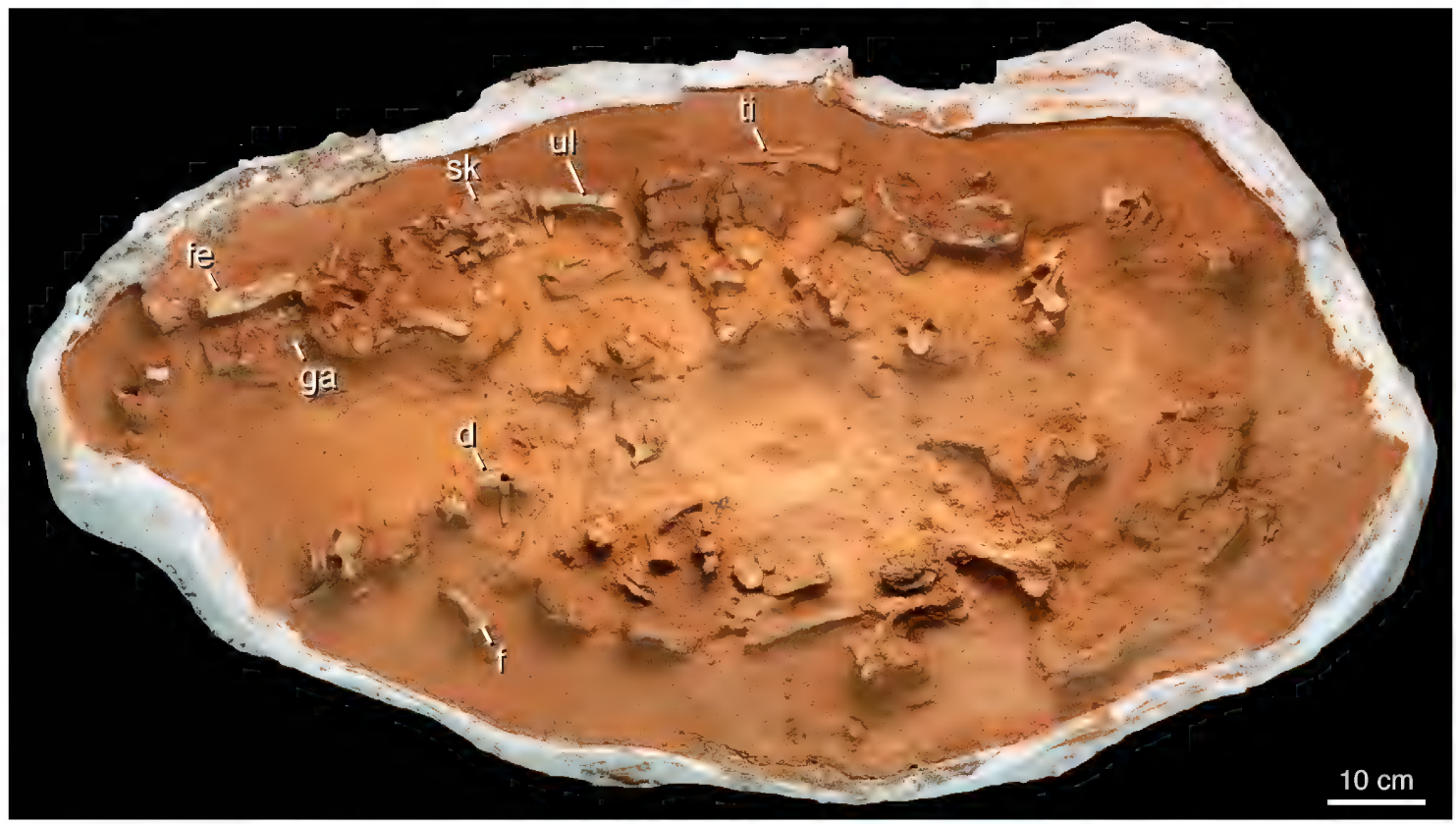

FIG. 14. Skeleton of Haya griva, IGM 100/3661. Note the scattered distribution of skeletal elements. Further preparation and study of this specimen is ongoing. Abbreviations in appendix 1.

No posterior supraorbitals are preserved in IGM 100/2017. In IGM 100/3178, IGM 100/2014, and IGM 100/2019 (figs. 6, 7, 9), a small, rod-shaped, slightly ventrally curved posterior supraorbital overlaps the anterior margin of the postorbital. The posterior supraorbital lies in the same plane as the anterior supraorbital, but does not contact it. Among early-diverging neornithischians, only Haya, Agilisaurus, Isaberrysaura, and Thescelosaurus possess posterior supraorbitals (Peng, 1992; Makovicky et al., 2011; Boyd, 2014; Salgado et al., 2017). The holotype of Changchunsaurus possesses a small, broken, rodlike bone adhering to the postorbital (Zan et al., 2005; Jin et al., 2010). It is unclear whether this represents a fragment of the postorbital or a posterior supraorbital. In contrast to Haya, the anterior and posterior supraorbitals of Agilisaurus and Thescelosaurus likely contacted or nearly contacted one another (Peng, 1992; Boyd, 2014). Uniquely, the single anterior supraorbital of Dryosaurus may have spanned the entire orbit to contact the postorbital (Galton, 1983). Many basal neornithischian taxa (Jeholo- saurus, Hexinlusaurus, Orodromeus, Zephyrosaurus) possess an occasionally rugose anterior projection on their postorbital (Sues, 1980; He and Cai, 1984; Scheetz, 1999; Barrett et al., 2005; Barrett and Han, 2009), which in Haya and Thescelosaurus is the attachment site for the posterior supraorbital. Thus it remains possible that in the future these other taxa will be found to have posterior supraorbitals.

Sclerotic Ossicles: The partial sclerotic ring of IGM 100/2017 is preserved lying against the pterygoid within the right orbit (fig. 3). The left orbit also contains a partial sclerotic ring. In IGM $100 / 3178$ (fig. 7), portions of at least 10 sclerotic ossicles are visible, though these do not make up a full ring. The individual sclerotic ossicles are not as well defined as in IGM 100/2017, but the ring is more three dimensionally preserved overall, and may lie in or just ventral to the approximate life position. The ossicles are simple, extremely thin, roughly rectangular imbricated sheets of bone. The rings are too poorly preserved to determine whether "+" (completely overlapping at both ends) or “_” 


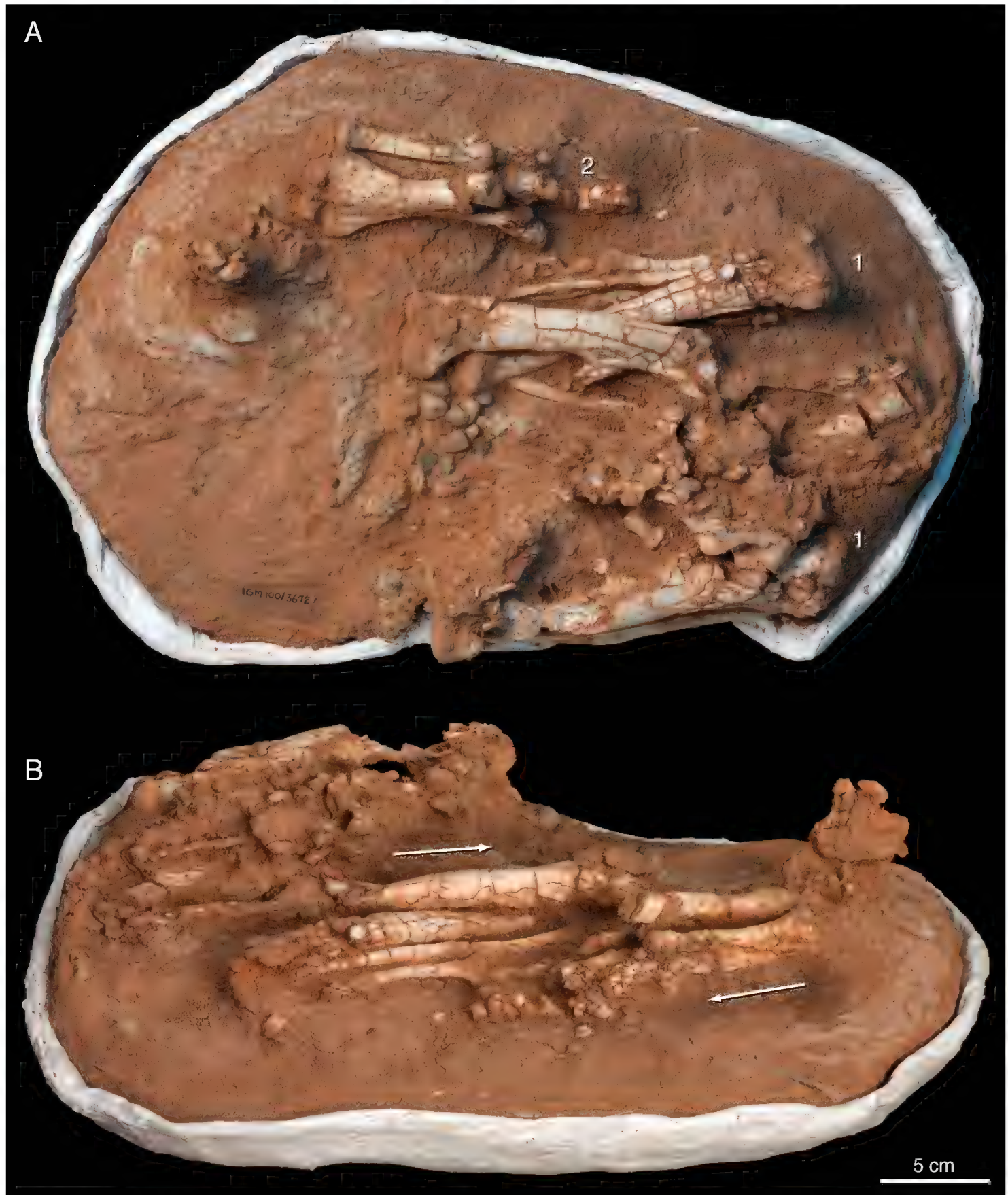

FIGURE 15. IGM 100/3672, containing the remains of two Haya griva individuals (labeled 1 and 2), represented by three pedes in $\mathbf{A}$, dorsal and $\mathbf{B}$, oblique lateral view. The arrows in $\mathbf{B}$ indicate the pedes of the two individuals pointing in opposite directions. 
(completely overlapped at both ends) ossicles (Russell, 1940) were present. Similar complete or near-complete sclerotic rings are known for Agilisaurus, Hypsilophodon, and Parksosaurus (Galton, 1973, 1974a; Peng, 1992).

SuPRAOCCIPITAL: The supraoccipital is an anteroposteriorly short, anterodorsally inclined, single median element (fig. 18). It is roughly pyramidal. The supraoccipital forms most of the dorsal margin of the foramen magnum, as is typical of basal neornithischian taxa other than Thescelosaurus, in which its contribution is reduced (Galton, 1989; Brown et al., 2011), and iguanodontians (except for dryosaurids, Rhabdodon, and Camptosaurus) in which it is completely excluded (Norman, 2004; Hübner and Rauhut, 2010). Its dorsal process is mediolaterally narrower, with a more pointed dorsal edge in posterior view than those of Lesothosaurus, Orodromeus, Thescelosaurus, Tenontosaurus, Dryosaurus and Dysalotosaurus (Galton, 1983; Scheetz, 1999; Hübner and Rauhut, 2010; Brown et al., 2011; Boyd, 2014; Porro et al., 2015; Thomas, 2015). The dorsal process is separated from the remainder of the bone by a postmortem break in IGM 100/2017. The parietals contact and overhang the supraoccipital dorsally, and the fused exoccipitals/opisthotics abut it laterally on both sides. The supraoccipital appears to be unfused to the parietals and the opisthotics in IGM 100/2016, IGM 100/2017, and IGM 100/2019 (figs. 10E, 18). The lateral processes that contact the opisthotics are proportionally taller dorsoventrally in posterior view than those of Thescelosaurus (Brown et al., 2011; Boyd, 2014). A faint midline keel (the nuchal crest of Sues, 1980) is present on the supraoccipital as in many early-diverging neornithischians, though it is reduced or absent in Orodromeus (Scheetz, 1999). This crest diminishes ventrally and disappears dorsal to the margin of the foramen magnum. Unlike in Hypsilophodon (Galton, 1974a), the dorsal margin of the supraoccipital is not notched at its midline contact with the parietal (Makovicky et al., 2011). A Haya specimen, IGM $100 / 2019$, possesses a small hole near the ventral

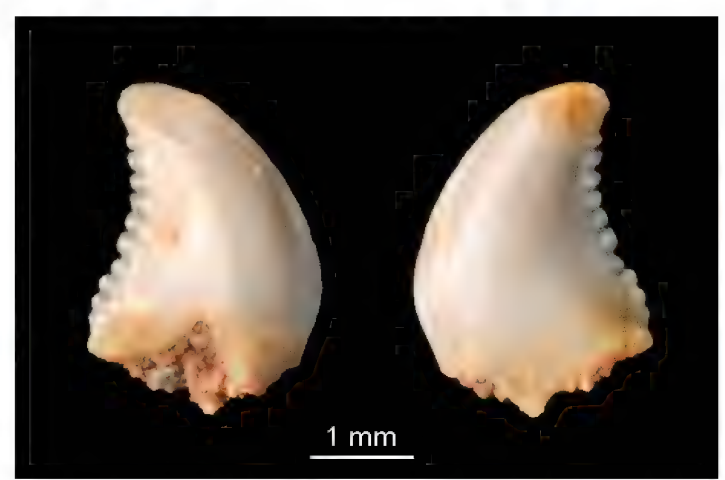

FIGURE 16. Troodontid tooth found in matrix from the IGM $100 / 3672$ jacket.

border of the supraoccipital that recalls the supraoccipital foramen of Thescelosaurus assiniboiensis (Brown et al., 2011), but its nature is unclear, possibly either biological or taphonomic in origin. The articulated nature of the Haya specimens makes it difficult to assess the extent of the supraoccipital contribution to the lateral wall of the braincase, and whether or not an epiotic was present (Sereno, 1991; Butler, 2010). However, its contribution to the lateral wall appears negligible in CT slices, and is probably less than in Hypsilophodon, Thescelosaurus, and Tenontosaurus (Galton, 1989; Brown et al., 2011). Features of the ventral surface of the supraoccipital, such as the fossa subarcuta that receives the floccular lobe of the cerebellum (Galton, 1989), cannot be readily observed.

Exoccipital/Opisthotic: The exoccipitals form the lateral borders of the foramen magnum and are completely fused to the opisthotics in IGM 100/2017, as confirmed in CT slices. As for all early-diverging neornithischians and ornithopods, with the possible exception of Zephryosaurus (Sues, 1980), there is no visible suture between the exoccipital and opisthotic in IGM $100 / 2017$ (fig. 18A). However, a suture is visible in IGM 100/2016 (fig. 10A), possibly indicating its immature status, as the two bones are thought to fuse very early during ontogeny in most dinosaurs (Hübner and Rauhut, 2010). Anteriorly, the opisthotic articulates with the prootic. The exoccipitals are raised, fingerlike projections that 


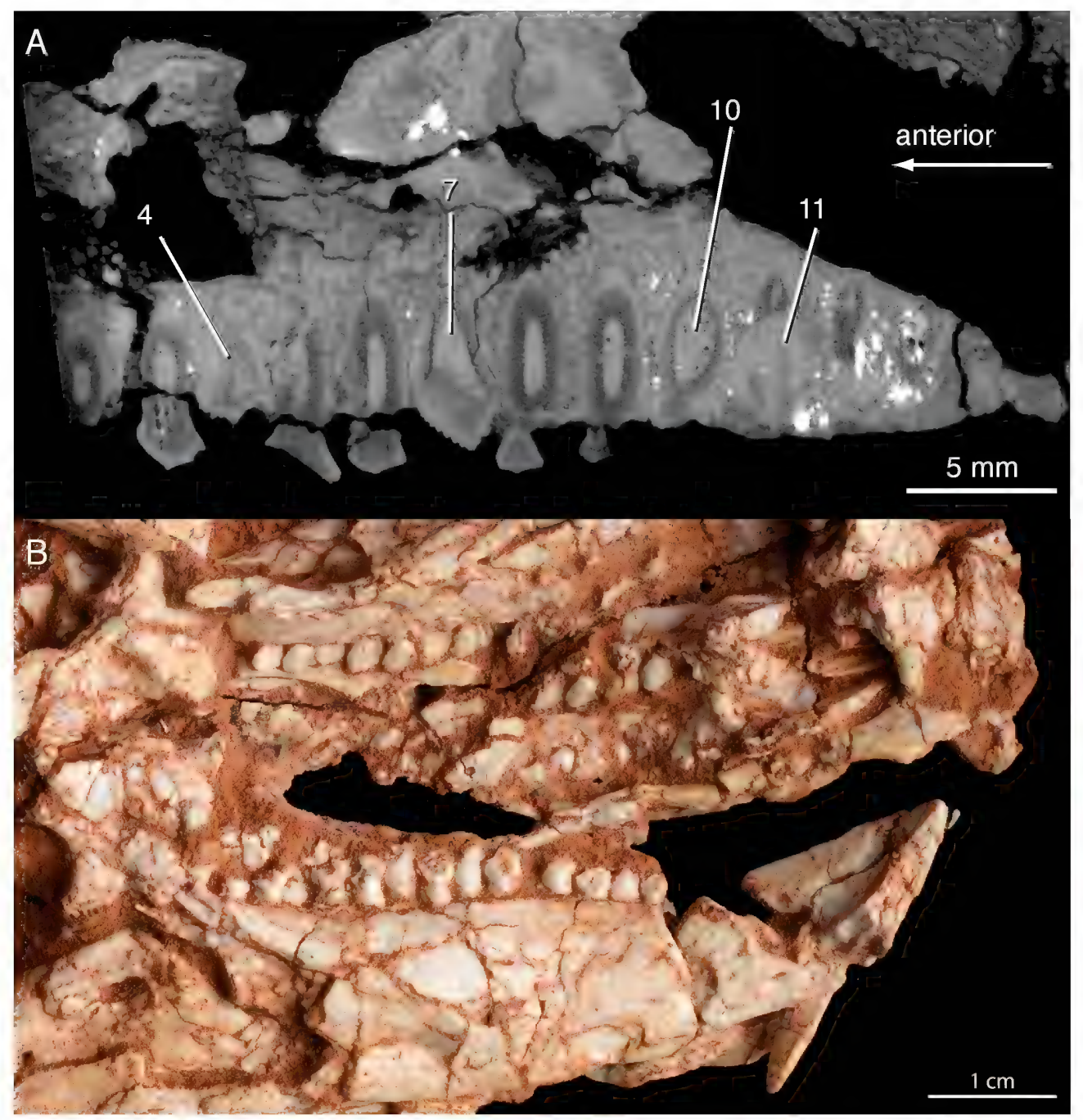

FIG. 17. A, Sagittal CT slice through the right maxilla of IGM 100/2017, showing four replacement teeth with their sequential anterior to posterior positions indicated. B, Maxillary and dentary teeth of IGM 100/2014 in right lateral view. See figure 26B for teeth from the left side of this specimen.

extend ventrally to contact the basioccipital along its dorsal margin, contributing to the occipital condyle. In IGM 100/2017, a smaller fingerlike process extends ventrally along one third the lateral surface of the exoccipital (fig. $18 \mathrm{~A})$. In lateral view, it is located just posterodorsal to the foramen for the hypoglossal nerve (c.n. XII). This process may be the boss for articulation with the proatlas. If so, it is positioned much more ventrolaterally than in Thescelosaurus (Boyd, 2014). IGM 100/2016 and IGM 100/2019 lack this process. The form and location of this process may be autapomorphic for Haya griva, as it does not match that for any other early-diverging neornithischian or ornithopod, and most lack such a process. However, given that it is currently present only in the holotype, it is difficult to determine whether this process is a typical feature of Haya griva. The left exoccipital and basioccipital are separated slightly by matrix in IGM 100/2017 (visible in CT slices), whereas the two bones are more tightly joined on the right 


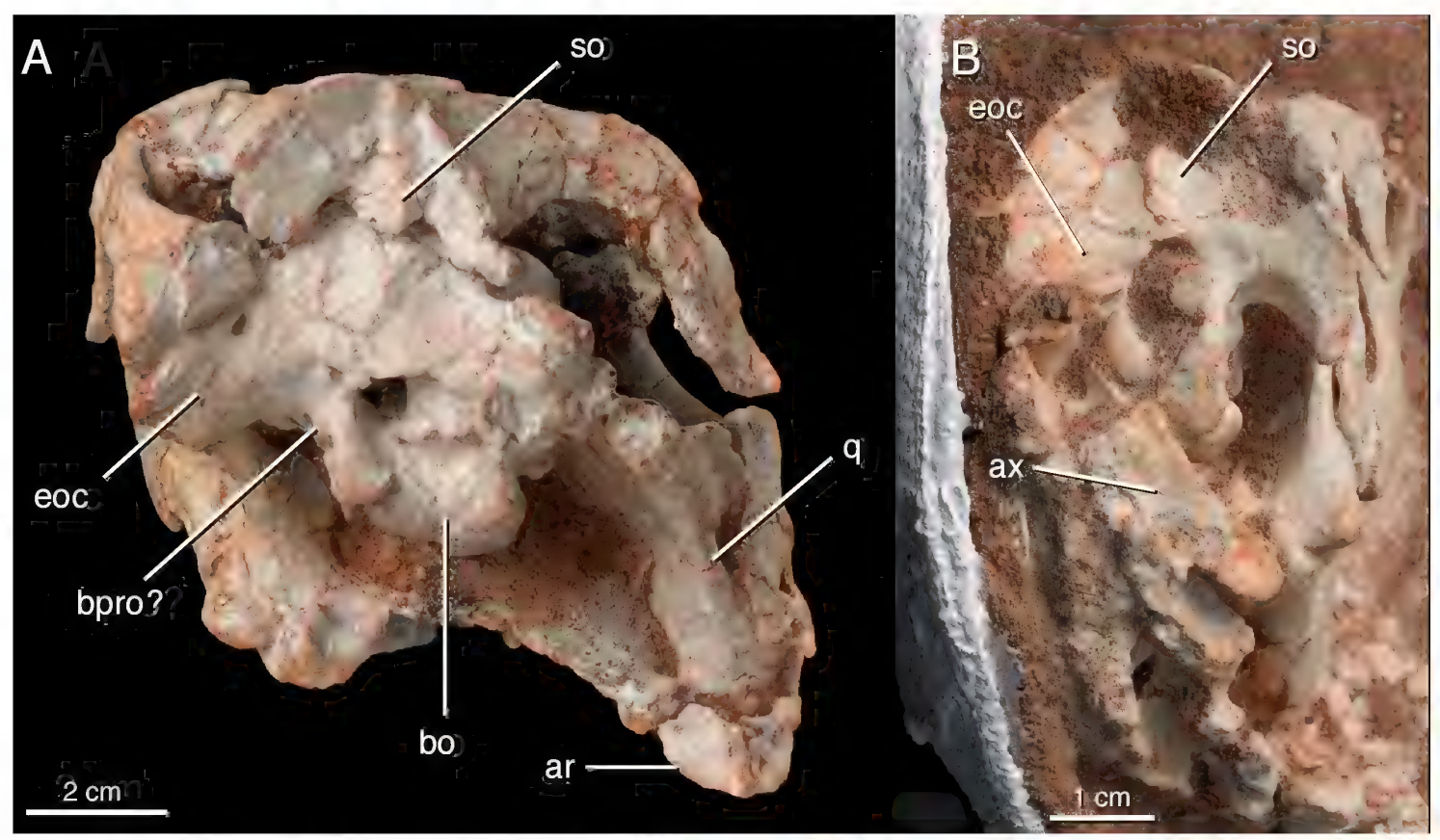

FIG. 18. Occipital region of Haya griva, A, IGM 100/2017 and B, IGM 100/2019. Abbreviations in appendix 1.

side, with the suture visible as a line of higher density separating them on the CT slices. The exoccipital and basioccipital are possibly unfused in IGM 100/2016 (fig. 10), and possibly fused with a visible suture in IGM 100/2019 (fig. 18B, 19). Two foramina for the hypoglossal nerve (c.n. XII) perforate the exoccipital within a deep lateral sulcus posterior to the crista tuberalis. The foramina lay directly in line with one another, anteroposteriorly. The foramen for the vagus nerve (c.n. X) perforates the exoccipital anterodorsally to the two hypoglossal openings. The vagus foramen faces almost directly posteriorly, in contrast to those of Thescelosaurus and Dysalotosaurus, which face more laterally (Sobral et al., 2012; Boyd, 2014). In IGM 100/2019, two or possibly three foramina for cranial nerves are visible on the lateral side of the right exoccipital, though it is not clear whether they represent both hypoglossal foramina or one hypoglossal foramen and the vagus foramen (fig. 19).

The dorsal portions of the opisthotic expand laterally as the paraoccipital processes. The paraoccipital processes are dorsoventrally broad and pendant, as revealed by the complete paraoccipital processes of IGM 100/2019 (fig. 18B, 19). Pendant paraoccipital processes are present in Zephyrosaurus, Thescelosaurus, Tenontosaurus and absent in Lesothosaurus, Jeholosaurus, Hypsilophodon, and Gasparinisaura, which have expanded, but less ventrally directed margins of their paraoccipital process. There is a broad depression on the paraoccipital process near the dorsolateral corner of the foramen magnum, probably the insertion site for $\mathrm{m}$. rectus capitis posterior (Ostrom, 1961). The lateral borders of the paraoccipital processes are striated, possibly for the insertions of all or some of the following muscles: M.obliquus capitis magnus, $M$. transversalis capitis, M. rectus capitis lateralis, M. episternocleidomastoideus, and M. depressor mandibulae (Ostrom, 1961; Tsuihiji, 2010). The posttemporal foramen, for passage of vena capitis dorsalis (Bruner, 1907; Janensch, 1936; Norman et al., 2004; Sobral et al., 2012), pierces the paraoccipital process dorsally near its contact with the squamosal, as in Heterodontosaurus, Zephyrosaurus, Jeholosaurus, Hypsilophodon, 


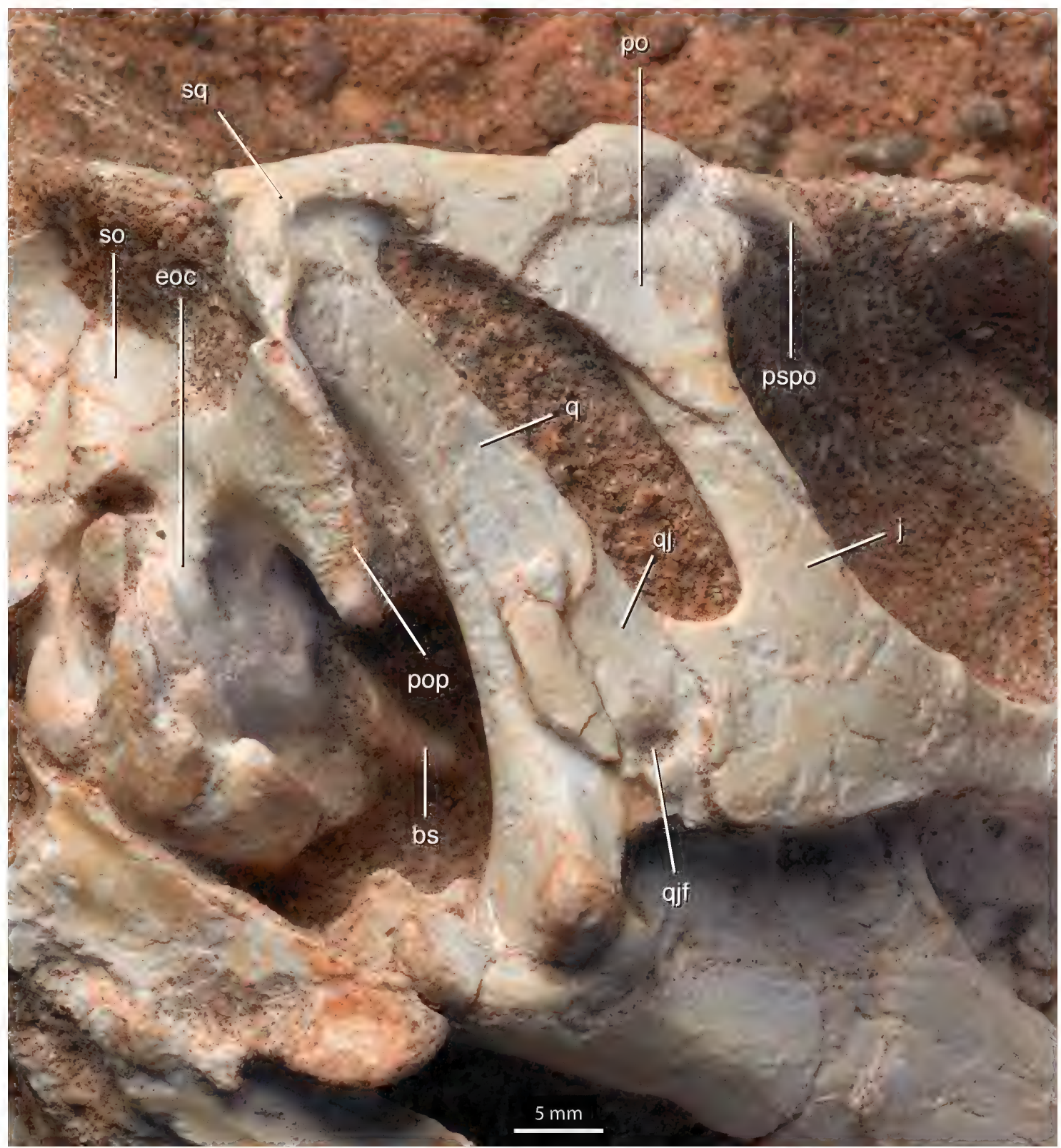

FIG. 19. Braincase of IGM 100/2019 in oblique right lateral view. Abbreviations in appendix 1.

Gasparinisaura, and possibly Lesothosaurus (Galton, 1974a; Sues, 1980; Sereno, 1991; Coria and Salgado, 1996; Barrett and Han, 2009; Norman et al., 2011; Porro et al., 2015). In contrast, both Thescelosaurus species and Dysalotosaurus have an open groove for vena capitis dorsalis on the dorsal edge of the paraoccipital process (Galton,
1989; Brown et al., 2011; Sobral et al., 2012; Boyd, 2014).

BASIOCCIPITAL: The basioccipital is a roughly cubic bone with a pronounced ventral flexure that gives it the appearance of an inverted saddle (figs. 4B, D, 10, 18). Dorsally, the basioccipital articulates with the exoccipital/opisthotic and 


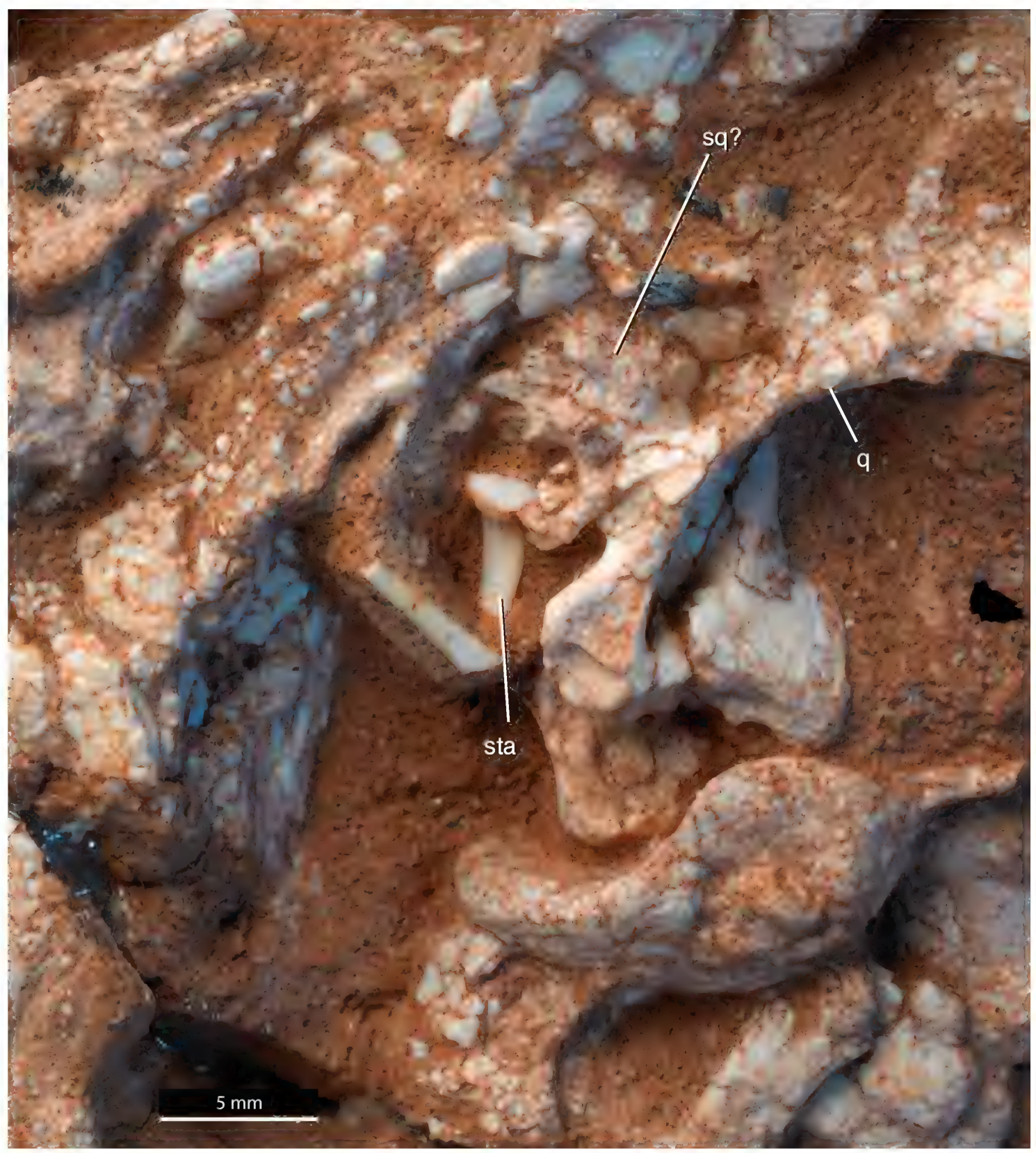

FIG. 20. Otic region of IGM 100/2014. Abbreviations in appendix 1.

prootic, and anteriorly with the basisphenoid along the basal tubera. The dorsal surface of the basioccipital is flat to slightly concave, mediolaterally. The basioccipital contributes the major ventral portion of the reniform occipital condyle (Makovicky et al., 2011), and thus forms the ven- tral border of the foramen magnum. A prominent lip encircles the condyle and divides it from the rest of the bone. As in Zephyrosaurus and Thescelosaurus (Sues, 1980; Boyd, 2014), a faint keel runs anteroposteriorly along the ventral midline, near the contact with the basisphenoid. 


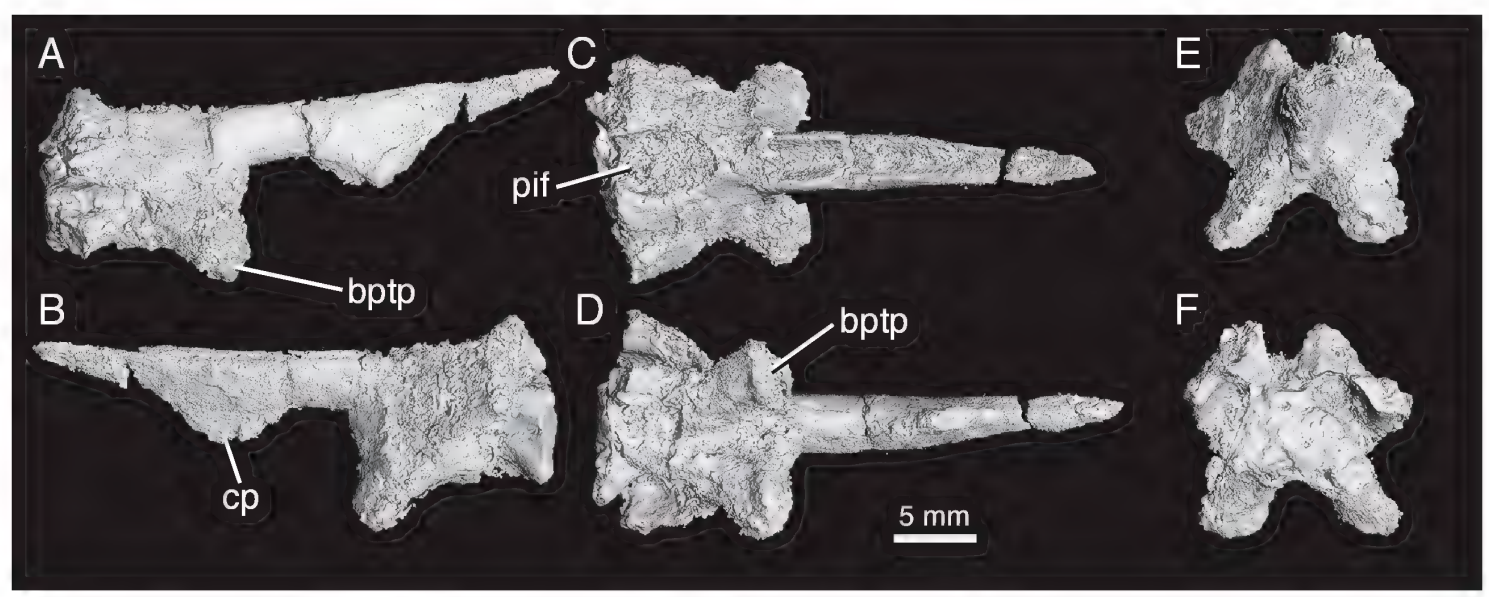

FIG. 21. CT reconstruction of the basisphenoid of IGM 100/2017 in A, right lateral, B, left lateraL, C, dorsal, $\mathbf{D}$, ventral, E, anterior, and $\mathbf{F}$, posterior views. Abbreviations in appendix 1.

This keel is larger relative to the size of the basioccipital in IGM 100/2016 (fig. 10A) than in IGM 100/2017 (fig. 4B). The basioccipital and basisphenoid are unfused in IGM 100/2017. The suture with the basisphenoid is clearly visible in IGM 100/2016; whether the two elements are fused is difficult to determine.

Prootic: The anterior portions of this element are eroded in IGM 100/2017 and IGM $100 / 2016$. It is not fused to the exoccipital/opisthotic in either specimen. Though displaced slightly, the region containing the foramen metoticum and fenestra ovalis between the prootic and exoccipital/opisthotic is visible in IGM $100 / 2017$, though any crista interfenestralis separating the two has probably eroded. The posterior half of the bone contains a slitlike foramen bounded by a ridge anteriorly. This may be the foramen for the facial nerve (c.n. VII), given its similar shape, location, and orientation to that of Thescelosaurus (Boyd, 2014).

STAPES: The otic region of IGM 100/2014 contains a small, rod-shaped element with a flat plate on its dorsally facing end (fig. 20). The flat plate is probably the footplate of the stapes. Despite its slight displacement within the crushed skull, its relative size, location, and morphology are all consistent with the stapes of Lesothosaurus and Thescelosaurus, as well as other dinosaurs and extant reptiles (Colbert and Ostrom, 1958; Sereno, 1991; Galton and Upchurch, 2004; Boyd, 2014). We concur with Boyd (2014) in regarding the "stapes" of Jeholosaurus described by Barrett and Han (2009) as a hyoid element.

Basisphenoid/Parasphenoid: The basisphenoid and parasphenoid are fused, as in most dinosaurs, and together they contribute to the ventral floor of the braincase (fig. 21). The body of the basisphenoid is anteroposteriorly concave ventrally, and narrows along its middle, flaring both at the basipterygoid processes anteriorly and at its contributions to the basal tubera along its contacts with the basioccipital posteriorly. The basisphenoid is proportionally longer in Heterodontosaurus than in Haya and other neornithischians (Norman et al., 2011). As in Thescelosaurus, the parasphenoid is at the same level as the dorsal surface of the basisphenoid (Boyd, 2014), instead of offset ventrally from it in lateral view as in Dysalotosaurus (Sobral et al., 2012). The cultriform process extends from the parasphenoid anteriorly along the midline (fig. 21). The short, dorsoventrally deep keel of the cultriform process is restricted to the middle one third of the cultriform process and is separated from the body of the basisphenoid by a distinct "neck" in lateral view, unlike Thescelosaurus, in 


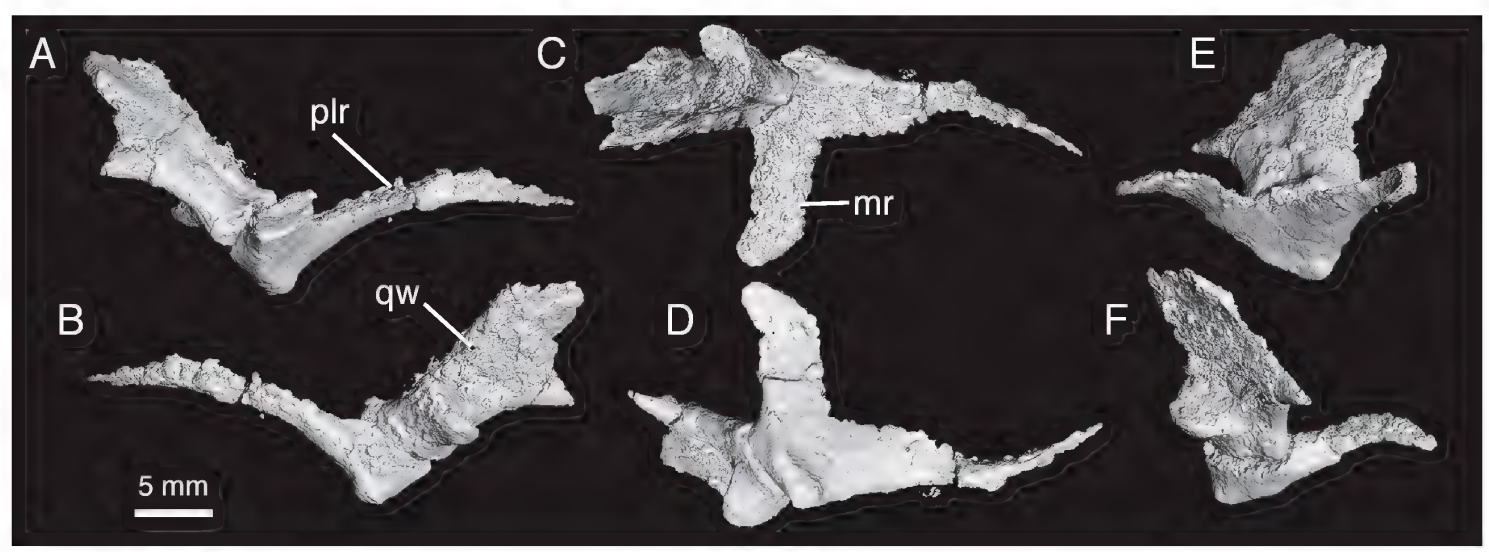

FIG. 22. CT reconstruction of the right pterygoid of IGM 100/2017 in A, lateral, B, medial, C, dorsal, D, ventral, $\mathbf{E}$, anterior, and $\mathbf{F}$, posterior views. Abbreviations in appendix 1

which the keel merges more gradually with the body of the basisphenoid (Boyd, 2014), and Lesothosaurus, which lacks such a keel (Sereno, 1991; Porro et al., 2015). The cultriform process contains a broad groove dorsally. A faint transverse ridge separates this groove from the pituitary fossa posteriorly (fig. 21). The rounded, knoblike basipterygoid processes extend anteroventrally, as in Lesothosaurus, Heterodontosaurus, and Thescelosaurus (Sereno, 1991; Norman et al., 2011; Boyd, 2014; Porro et al., 2015), but in contrast to the more ventrally directed basipterygoid processes of Zephyrosaurus and Dysalotosaurus (Sues, 1980; Sobral et al., 2012). They are also shorter and stouter than the elongate basipterygoid processes of Tenontosaurus (Thomas, 2015). The anterolateral processes of the basisphenoid appear to be shorter in Haya than in Thescelosaurus (Boyd, 2014), but they may be incompletely preserved in IGM 100/2017. Likewise, the preotic pedants may be less pronounced in Haya than in Thescelosaurus (Boyd, 2014), but again it is not clear whether this is a biological or taphonomic difference. In IGM 100/2016, there is a small, medioventrally facing foramen or circular depression at the base of the left basipterygoid process in ventral view. The homology of this structure is unclear, as its position does not correspond exactly to any similar foramen or depression in other basal neornithischians for which this anatomical region is preserved. However, it may represent an opening of the Vidian canal for passage of the internal carotid artery and the palatine branch of the facial nerve (c.n. VII) (Ostrom, 1961).

LATEROSPHENOID: Little is preserved of this element in IGM 100/2017; it is difficult to discern even in CT slices.

Pterygoid: The pterygoid is divided into a sheet- to rodlike palatine ramus, tabular mandibular ramus, and sheetlike quadrate wing (or ramus), all roughly orthogonal to one another (fig. 22). The two pterygoids are closely appressed along part of their palatine rami anteriorly, but are separated by a narrow interpterygoid vacuity posteriorly. The pterygoid tapers to form a narrow palatine ramus that arches upward to contact the vomer anteriorly, near the level of the base of the lacrimal (Makovicky et al., 2011). The palatine ramus is relatively narrower than that of Lesothosaurus (Sereno, 1991; Porro et al., 2015), but not as laterally compressed as that of Thescelosaurus (Boyd, 2014). The anterior end of the palatine ramus also does not exhibit the dorsoventral expansion seen in Thescelosaurus (Boyd, 2014). The palatine ramus contacts the palatine anterolaterally. As in Changchunsaurus (Jin et al, 2010), the quadrate wing of the pterygoid extends dorsally to receive the basipterygoid processes of the basisphenoid within a U-shaped trough (fig. 


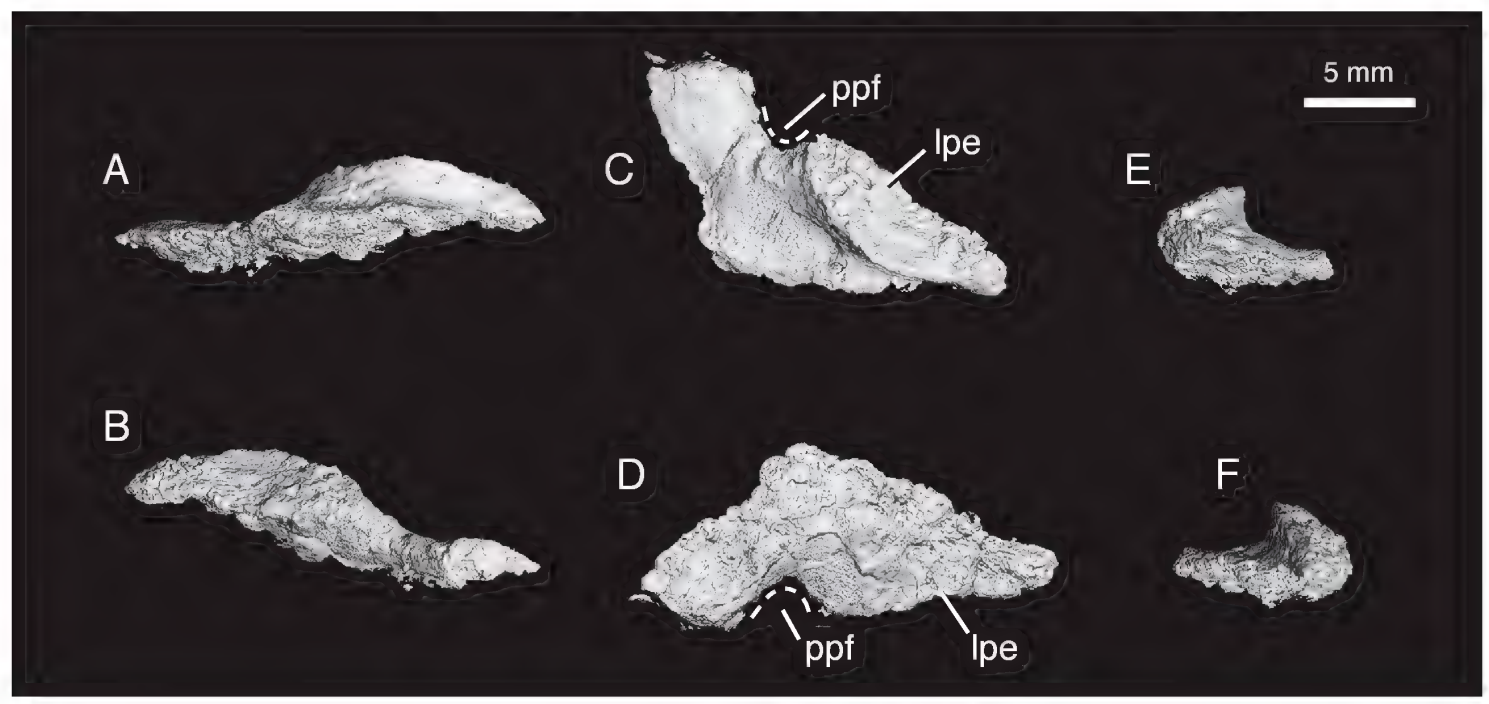

FIG. 23. CT reconstruction of the right ectopterygoid of IGM 100/2017 in A, lateral, B, medial, C, dorsal, D, ventral, $\mathbf{E}$, anterior, and $\mathbf{F}$, posterior views. Abbreviations in appendix 1.

4B, D). As noted by Makovicky et al. (2011), this articulation is situated dorsal to the union of the three pterygoid rami, as in Heterodontosaurus (Norman et al., 2011). This contrasts with Jeholosaurus, Changchunsaurus, and Thescelosaurus, in which the articulation with the basipterygoid process is nearer the junction of the rami (Barrett and Han, 2009; Jin et al., 2010; Boyd, 2014). The quadrate wing of the pterygoid extensively overlaps the pterygoid wing of the quadrate laterally. These contacts are well exposed in IGM $100 / 2016$ (fig. 10A). A thickened ridge is present at the junction between the quadrate ramus and the remainder of the pterygoid, but there is no pronounced pterygoid flange like that of Heterodontosaurus (Norman et al., 2011). As in Thescelosaurus, the tapering lateral edge of the mandibular ramus is deflected posteriorly, in contrast to the squared-off mandibular rami of Changchunsaurus and Hypsilophodon. Similar to the condition in those three taxa, the dorsal surface of the mandibular ramus is extensively overlapped by the ectopterygoid (Galton, 1974a; Jin et al., 2010; Boyd, 2014; fig. 4B, D). Because of this, the mandibular ramus does not border the postpalatine (= pterygopalatine or suborbital) fenestra as it does in Hypsilophodon (Galton, 1974a), though Makovicky et al. (2011) suggest that it does so in Changchunsaurus. However, its contribution to the border appears very minimal in Changchunsaurus (Jin et al., 2010: fig. 6B), and is similar overall to the condition in Haya and Thescelosaurus (Boyd, 2014).

ECtOPTERYGoid: The ectopterygoid shares extensive posterior and medial contacts with the pterygoid, and together with the palatine, completely excludes the pterygoid from the margin of the postpalatine (= pterygopalatine or suborbital) fenestra. The ectopterygoid is more extensively exposed in ventral view in Haya, Thescelosaurus (Boyd, 2014), and Hypsilophodon (Galton, 1974a) than in Lesothosaurus (Porro et al., 2015). As in Lesothosaurus and Changchunsaurus, but in contrast to Thescelosaurus, the ectopterygoid contacts the palatine and the palatal process of the pterygoid at a triple junction (Jin et al., 2010; Boyd, 2014; Porro et al., 2015; fig. 4B, D). The posterolateral corner forms a thickened, tablike, slightly ventrally deflected lateral process of the ectopterygoid (fig. 23). This lateral process contacts the maxilla immediately posterior to the toothrow (fig. 4), and though this process also contacts the jugal (Makovicky et al., 2011), the precise nature of the contact is 


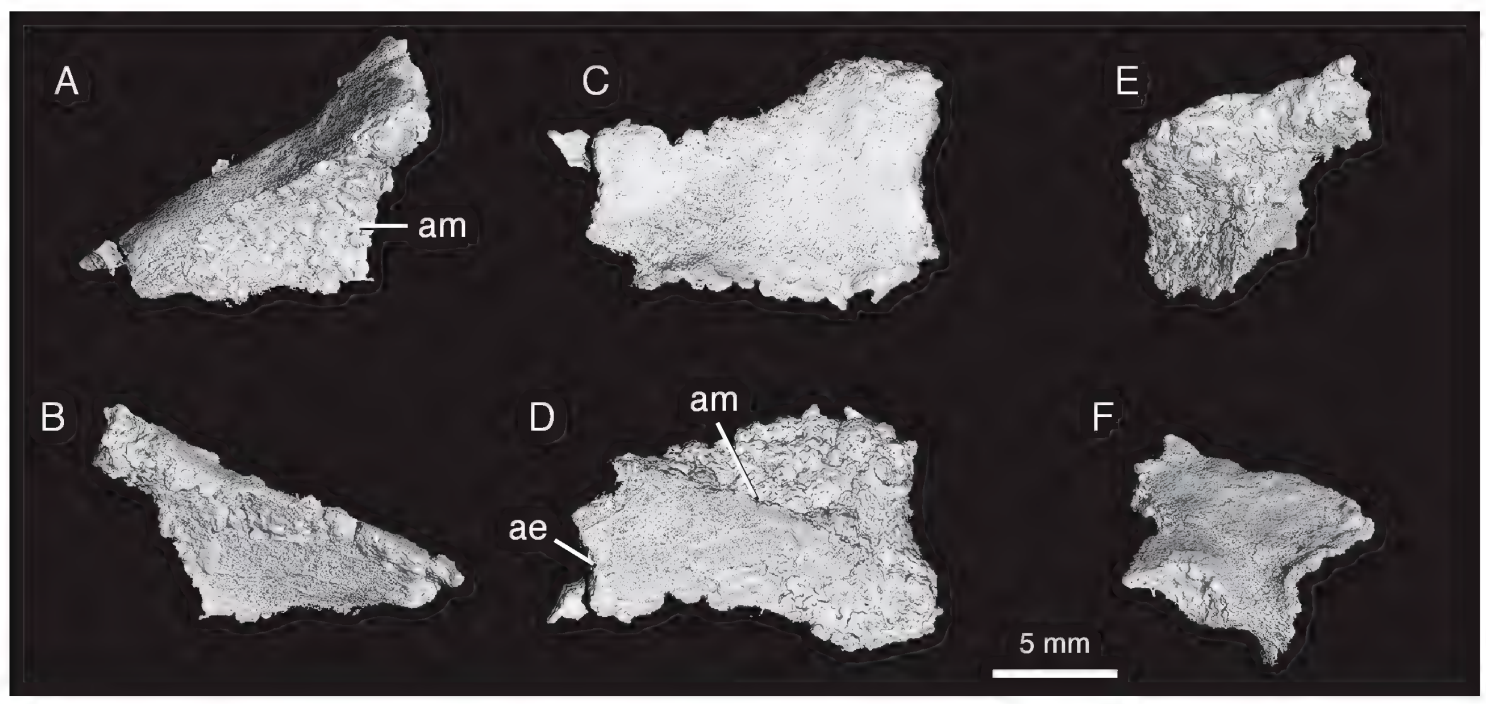

FIG. 24. CT reconstruction of the right palatine of IGM 100/2017 in A, lateral, B, medial, C, dorsal, D, ventral, $\mathrm{E}$, anterior, and $\mathrm{F}$, posterior views. Abbreviations in appendix 1.

difficult to discern. There is a groove on the dorsal surface of the lateral process through which the pterygoideus muscles may have passed (Makovicky et al., 2011) from their likely origin on the pterygoid and/or palatine to their insertion on the mandible, as in extant sauropsids (Holliday, 2009). Epipterygoids do not seem to be present, as is the case for other basal neornithischians (see Boyd, 2014, for reidentification of the "epipterygoids" of Jeholosaurus; Barrett and Han, 2009).

Palatine: Palatines are partially exposed in IGM 100/2017. As in most early-diverging neornithischians, the palatines are anterodorsally inclined, forming a vaulted palatal roof (Norman et al., 2004). The palatine walls the anteromedial portion of the orbit. It is a broad, dorsoventrally compressed bony sheet that articulates with the maxilla laterally along the posterior third of the toothrow (figs. $4 \mathrm{~B}, \mathrm{D}, 24$ ). The palatine is anteroposteriorly longer than that of Thescelosaurus (Boyd, 2014). Medially, it contacts the palatal rami of the pterygoid and the posterior end of the vomer (fig. 4B, D). The cultriform process of the parasphenoid also contacts both palatines along their midline contact, as in Lesothosaurus and Thescelosaurus (Boyd, 2014; Porro et al.,
2015). Due to obscuring matrix, it is not clear whether the palatines contact each other along their midlines. Contact with the pterygoid is less extensive than in Lesothosaurus (Porro et al., 2015). In contrast to Lesothosaurus and Hypsilophodon (Galton, 1974a), there is no palatinelacrimal contact. Nor does the palatine articulate with the jugal as in Lesothosaurus and Thescelosaurus (Boyd, 2014; Porro et al., 2015). Instead, the maxilla diverts the palatine medially along their contact, preventing the palatine from reaching either the lacrimal or jugal. The palatine does nearly meet both lacrimal and jugal near the lacrimal-jugal joint. The palatine forms the anteromedial corner of the postpalatine (= pterygopalatine or suborbital) fenestra (fig. 4B, D).

Vomer: Only portions of the vomer are externally visible in IGM 100/2017. As in Hypsilophodon (Galton, 1974a) and Thescelosaurus (Boyd, 2014), the vomers form two separate compressed sheets posteriorly that join to form a single median rod-shaped element that tapers slightly anteriorly (fig. 25). The posterior ends of the vomers overlap the palatal rami of the pterygoids dorsally, as is typical of most dinosaurs and possibly plesiomorphic for Archosauria (Sereno, 1991). In Haya, this contact occurs ventral to the 


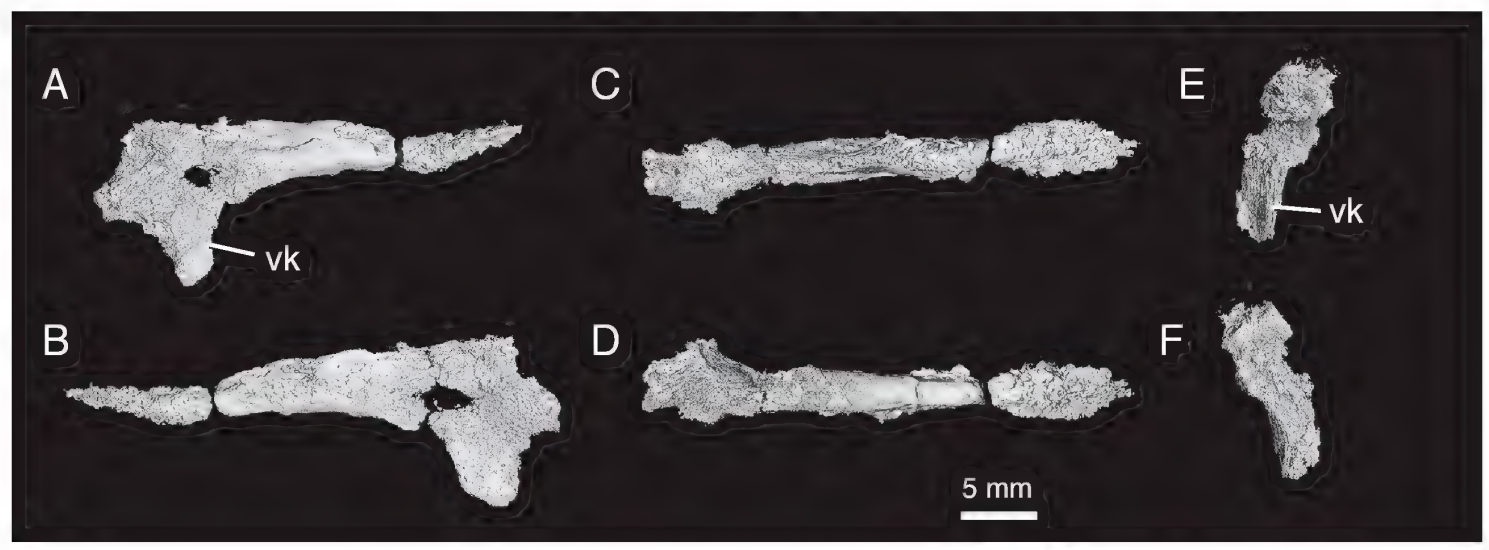

FIG. 25. CT reconstruction of the vomer of IGM 100/2017 in A, right lateral, B, left lateral, C, dorsal, D, ventral, $\mathbf{E}$, anterior, and $\mathbf{F}$, posterior views. Abbreviations in appendix 1.

position of the lacrimal. Posteriorly, the vomers also form a ventral keel that overlaps and extends below the anterior ends of the palatal rami of the pterygoids (fig. 25). The vomers of Thescelosaurus may form a similar posterior keel (Boyd, 2014: fig. 9H). Such a keel is also present, but less pronounced, in Tenontosaurus (the "median ventral ridge" of Thomas, 2015). The posterior wings of the vomers contact the palatines, as in Hypsilophodon and Tenontosaurus (Galton, 1974a; Thomas, 2015), but in contrast to Lesothosaurus and Thescelosaurus (Boyd, 2014; Porro et al., 2015). Only the middle portion of the anterior process of the vomer is concave ventrally, in contrast to the dorsoventrally thinner, more extensively concave vomer of Tenontosaurus (Thomas, 2015). CT slices reveal that the vomer contacts the premaxillae anteriorly.

Hyold: As in most other ornithischians, the single preserved pair of ceratobranchials are slender, slightly curved, posteriorly tapering rods (Morschhauser and Lamanna, 2013) (fig. 4B, D). The anterior articular facets of each ceratobranchial are roughly ovoid, and it appears that an ovoid or slightly triangular cross section is maintained along the length of the elements. In IGM $100 / 2017$, the left ceratobranchial probably lies in its life position, articulating with the posteromedial corner of the pterygoid (fig. 4B, D). A nearly identical articulation with the pterygoid is present in
Changchunsaurus (Jin et al., 2010: fig. 6B). Their posterior ends are directed posterolaterally and rest near the articulars. The ceratobranchials resemble those of Jeholosaurus, Changchunsaurus, and Hypsilophodon (Galton, 1974a; Barrett and Han, 2009; Jin et al., 2010), being less strongly bowed than in Thescelosaurus (Boyd, 2014). The elements identified by Porro et al. (2015) as the ceratohyals of Lesothosaurus more closely resemble the ceratobranchials of other basal neornithischian taxa based on their rodlike, gently curved morphology. These bones are typically identified as first ceratobranchials in other dinosaurs, based on the highly ossified nature of these elements in extant reptiles (Fürbringer, 1922; Colbert, 1945; Ostrom, 1961). Hill et al. (2015) cautioned that they could instead represent second ceratobranchials in some cases, based on their morphological similarity to the simple, rodlike second ceratobranchials of ankylosaurs. The orientation of the first ceratobranchial relative to the pterygoid in Pinacosaurus (Hill et al., 2015: fig. 4) is very similar to that of the unidentified ceratobranchial relative to the pterygoid in Haya and Changchunsaurus. On the basis of this topological similarity, we tentatively interpret the ceratobranchial elements in Haya and Changchunsaurus as homologous to the first ceratobranchial of Pinacosaurus. As in hadrosaurs, there are no obvious muscle attachment sites for the hyoid musculature (Ostrom, 1961). 
Predentary: As in other early-diverging ornithischians, the predentary is a sharply triangular, dorsally concave, and slightly upturned bone that articulates with the anterior ends of both dentaries (figs. 3-5, 7, 9, 11). The predentary of Haya is less anteroposteriorly elongate than that of Changchunsaurus and Thescelosaurus (Jin et al., 2010; Boyd, 2014; Nabavizadeh and Weishampel, 2016), and is similar in overall shape in lateral view to that of Hypsilophodon (Galton, 1974a). The ventral process of the predentary is nearly twice as long as the lateral process as seen in lateral view, and its posterior end is bifid, as in Changchusaurus, Thescelosaurus, and Talenkauen (Novas et al., 2004; Jin et al., 2010; Boyd, 2014), but in contrast to Lesothosaurus and Jeholosaurus, in which this process is not forked (Barrett and Han, 2009; Porro et al., 2015). The predentary processes of heterodontosaurids are simpler yet, in comparison with those of Haya and other neornithischians, with their rudimentary lateral and ventral processes (Norman et al., 2011; Sereno, 2012; Nabavizadeh and Weishampel, 2016). A deep lateral groove extends the length of the predentary in lateral view and continues onto the dentary anterior to the toothrow. A pair of nutrient foramina is present on the left side of the oral surface of the predentary (fig. 9B). Vascular foramina are otherwise sparse on the predentary of IGM 100/2017, where the surface is largely smooth. As in other basal neornithischians, the predentary-dentary joint (PDJ) is an example of PDJ morphotype I, a relatively simple convex-concave articulation that likely allowed for a slight degree of axial dentary rotation during feeding (Nabavizadeh and Weishampel, 2016).

Dentary and Dentary Dentition: The dentary is a long ramus that accounts for nearly half the total length of the mandible (Makovicky et al., 2011) (figs. 3-7, 9-11). The posterior end bows laterally, and the anterior end bows medially, forming a gentle sigmoidal curve in ventral view. It articulates with the predentary anteriorly, the angular and surangular posteriorly, and the splenial, prearticular, and coronoid medially.
There is no remnant of an external mandibular fenestra between the dentary, surangular, and angular, as in other basal neornithischians, but in contrast to Lesothosaurus and Heterodontosaurus (Sereno, 1991; Norman et al., 2011; Porro et al., 2015). Other such openings in this region in Hypsilophodon, Thescelosaurus, and Gasparinisaura appear to be contained entirely within the surangular, and therefore likely represent surangular foramina rather than remnants of external mandibular fenestrae (for an alternative interpretation see Norman et al., 2004). The hole seen near the junction of the dentary, surangular, and angular of the right mandible of IGM 100/2017 is a broken opening (fig. 3A). The posteriormost portion of the dentary forms a narrow, dorsally deflected coronoid process. The anteriormost end tapers to a well-defined triangular process that steps down abruptly from the oral margin and articulates with the predentary dorsally and ventrally (figs. 3, 7, 9-11, 13J). As IGM 100/2016 lacks a predentary, it clearly exposes the concave, slightly laterally flaring facets on the dentary where it articulates with the predentary (fig. $10 \mathrm{~F})$. A prong extends medioventrally from the ventral edge of the dentary ramus to meet the ventral prongs of the predentary. As in Changchunsaurus, but in contrast to other early diverging neornithischians, the sharp oral edge of the predentary continues as a ridge lateral to the first two alveoli of the dentary (Jin et al., 2010; Makovicky et al., 2011; fig. 3). The shared presence of this feature with Haya means it is no longer autapomorphic for Changchunsaurus, as Jin et al. (2010) hypothesized. Posterior to this, the dentary toothrow is inset from the lateral surface of the bone and occludes with the maxillary teeth. The anterior third of the dentary slopes slightly ventrally (fig. 3), a condition previously thought unique to Thescelosaurus among neornithischians (Boyd, 2014). There is no lateral groove ventral to the toothrow-this feature remains autapomorphic for Changchunsaurus (Jin et al., 2010). At least five to six nutrient foramina are present on the lateral surface of the dentary ramus ventral to the toothrow in IGM 
100/3178 (not all foramina are well preserved in IGM 100/2017; fig. 7). Haya lacks the large foramen within the buccal emargination of the dentary seen in Changchunsaurus (Jin et al., 2010). The larger, paired mental foramina lie anterior and ventral to the nutrient foramina, situated within a continuation of the lateral groove of the predentary (fig. 3). The mental nerve (a branch of the inferior alveolar nerve, itself a branch of the mandibular division of the trigeminal nerve, c.n. V) likely passed through these foramina (Gilmore, 1909; Galton, 1974a). The lingual plate on the medial surface of the dentary is pierced by a row of ovoid replacement or "special" foramina (Edmund, 1957) ventral to the alveoli, similar to those of Changchunsaurus, Hypsilophodon, and many other ornithischians (Edmund, 1957; Galton, 1974a; Jin et al., 2010). In contrast to Haya, these foramina are slitlike and more dorsally directed in Fruitadens (Butler et al., 2012) and largely covered by the coronoid in Lesothosaurus (Sereno, 1991). Because of their size and close correspondence to the positions of the teeth, the special foramina were probably sites where the dental lamina invaded the lingual plate and formed new replacement teeth, which may have been supplied by blood vessels entering through each foramen (Edmund, 1957; LeBlanc et al., 2016). A different reconstruction of a hadrosaur dentary shows branches from the internal mandibular artery and nerve exiting, rather than entering, the foramina (Holliday, 2009: fig. 7). The function of the special foramina continues to be uncertain given their complex phylogenetic distribution and individual variation among dinosaurs, and their absence in other amniotes (LeBlanc et al., 2017). As in many amniotes, the deep Meckelian canal runs along the medial surface of the ventral margin of the dentary (figs. 4B, 10, 13J). The splenial covers this groove along much of its length.

In IGM 100/2017, the right dentary contains 16 alveoli and the left contains 15 , as confirmed by examining CT slices. This mirrors the asymmetry in the maxillary tooth counts, where the right maxilla has one more tooth position than the left maxilla. The dentary alveoli are damaged in IGM 100/2016 and only two teeth remain in or close to their life positions. Nevertheless, it is apparent that this morphologically juvenile skull possessed fewer dentary tooth positions than the holotype, possibly only 10 to 12 . IGM 100/2014 has 13-14 dentary teeth. Likewise, CT slices reveal that IGM $100 / 3178$ has at least 13 teeth in its left and right dentaries, an intermediate number that might suggest this specimen is at an intermediate ontogenetic stage between IGM 100/2016 and IGM 100/2017. However, the dentaries of IGM 100/3178 and IGM 100/2017 are nearly the same length, so the difference in tooth count is likely due instead to individual variation or taphonomic differences. The dentaries of both specimens are longer than those of IGM 100/2016, suggesting that the estimated lesser tooth count in this specimen truly reflects its earlier ontogenetic stage. Haya possesses more dentary teeth at maximum (16), among known specimens, than the typical 13 to 14 for currently known specimens of other noncerapodan neornithischians (Norman et al., 2004). Only Lesothosaurus, Agilisaurus, Hexinlusaurus, Kulindadromeus, Parksosaurus, and Thescelosaurus have more dentary teeth, with 17 to 20 . Future discoveries of ontogenetically more mature specimens may increase the maximum tooth counts for Haya or other taxa. Caution should be used in diagnosing taxa based on tooth number if only immature specimens are known. For much of the toothrows of IGM $100 / 2017$ and IGM 100/2014, the tooth margins are imbricate, with the distal sides of more anterior teeth overlapping the mesial sides of more posterior teeth. The teeth do not overlap in other places along the dentaries of the same specimens, though this could be due to postmortem shifting.

CT scans reveal that the five erupted teeth have single replacement teeth below them in the right dentary of IGM 100/2017: at the first, fourth, seventh, ninth, and 14th alveoli (counted from anterior to posterior) (fig. 26A). The erupted teeth at these positions are among the 


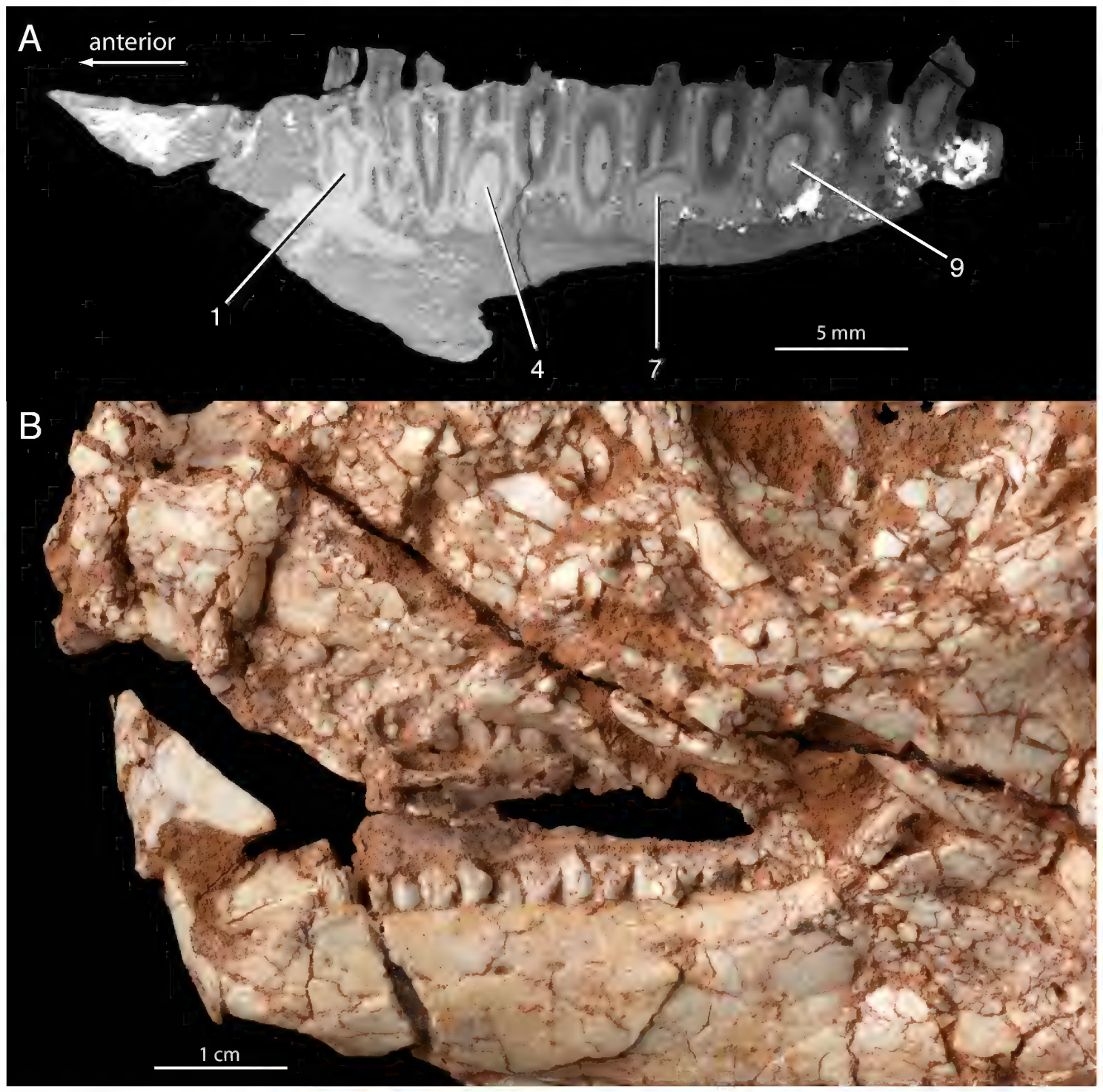

FIG. 26. A, Sagittal CT slice through the right dentary of IGM 100/2017, showing four replacement teeth with their sequential anterior to posterior positions indicated. B, Maxillary and dentary teeth of IGM 100/2014 in left lateral view. See fig. 17B for teeth from the right side of this specimen.

most heavily worn along the toothrow. Only the replacement tooth at the ninth alveolus has partially erupted, lingual to a heavily worn, erupted tooth. The left dentary contains replacement teeth at the third, sixth, ninth, and 12th alveoli; those at the third and sixth positions are partially erupted. This suggests that there is at least a rough alternating pattern of tooth replacement along the dentary in Haya, as in most reptiles (Edmund, 1960). However, the extent of tooth replacement in IGM 100/2017 alternates approximately every third tooth, as opposed to every other tooth in Parksosaurus (Galton, 1973), Hypsilophodon (Galton, 1974a), Tenontosaurus (Thomas, 2015), and most other dinosaurs (Edmund, 1960). IGM 100/2017 shows evidence 
for out-of-sync tooth-replacement waves between the left and right dentaries. The two anterior replacement teeth of the four present in the left dentary are erupted, whereas only one more posterior replacement tooth has erupted along the right dentary.

The anteriormost three dentary teeth are simple, with smaller and narrower crowns, as in Orodromeus (MOR 248), and in contrast to Thescelosaurus, in which the first two dentary teeth are not obviously reduced and may actually be larger than more posterior teeth (Boyd, 2014). As in Thescelosaurus, the anteriormost two or three dentary teeth did not occlude with the maxillary teeth (Boyd, 2014). Posterior to these, unworn dentary teeth possess triangular crowns with one prominent inflated apical denticle that forms a faint primary enamel ridge, and approximately four to six smaller denticles to each side of the central primary denticle (figs. 17B, 26B). Thinner ridges that do not reach the cingulum extend basally from the smaller denticles (Makovicky et al., 2011). There are typically between nine and 13 denticles in total. The denticles nearest the apical denticle occasionally take the form of small accessory denticles extending from the side of the apical denticle. Haya tooth crowns are symmetrical, unlike the asymmetrical crowns of Lesothosaurus, Othnielosaurus, and Drinker (Sereno, 1991; Galton, 2007), and the slightly asymmetrical crowns of Orodromeus with their curved primary ridges (D.E.B., personal obs.; Scheetz, 1999). The primary ridge is better developed in Haya than Othnielosaurus, where it may be absent (Galton, 2007). However, the primary ridge in Haya is not as sharp and well defined as that of Changchunsaurus, Orodromeus, and Hypsilophodon (D.E.B., personal obs.; Galton, 1974a 2007; Jin et al., 2010). Unlike Jeholosaurus and Changchunsaurus, the enamel ridges are not less developed on the dentary teeth than the maxillary teeth (Barrett and Han, 2009; Jin et al., 2010), having similar morphologies on both types of teeth in Haya. The cingulum is a broad U-shaped shelf, below which the tooth constricts greatly, down to the tubular root. In lingual view, the cingulum is thickest on the posterior side of the tooth. Overall, the dentary tooth crowns are very similar morphologically to those of Zephyrosaurus (Sues, 1980). The roots of the teeth are dorsoventrally straight to gently curved, as in Othnielosaurus and Thescelosaurus, but in contrast to the more strongly curved roots of Hypsilophodon, Dryosaurus, Zalmoxes, and Camptosaurus (Galton, 1974a, 1983, 2007; Weishampel et al., 2003; Boyd, 2014). Unlike heterodontosaurids, Orodromeus, Thescelosaurus, Tenontosaurus, and Dryosaurus (Hopson, 1980; Galton, 1983; Weishampel, 1984; Scheetz, 1999; Norman et al., 2011; Boyd, 2014), which often have double labial wear facets on each dentary tooth, Haya dentary teeth typically have single wear facets, though the heavy wear on the teeth of IGM 100/2017 may have obliterated the distinction between the facets. The position of the dentary teeth relative to the maxillary teeth suggests that at least some dentary teeth occluded with up to two maxillary teeth. This is similar to heterodontosaurids in which each maxillary tooth sometimes overlaps two dentary teeth. In Lesothosaurus, the occlusal pattern is more irregular (Sereno, 1991).

SURANGULAR: The surangular and its bounding sutures are best exposed in IGM 100/2014 (fig. 9B). In right lateral view, it is a sigmoidal bone that expands dorsoventrally into a broad sheet anteriorly (Makovicky et al., 2011). This sheet forms the dorsally directed coronoid process. The tapered anterior end articulates with the dentary. The surangular also articulates with the angular ventrally, and the prearticular and coronoid medially. Together with the dentary, the coronoid process of the surangular makes up the coronoid eminence. The surangular is more dorsoventrally expanded than in Lesothosaurus (Porro et al., 2015). Two foramina pierce the surangular; one ventral to the coronoid process, near the center of the broad anterior sheet (the surangular foramen), and another just anterior to the lateral process of the surangular (the "lateral process foramen"; Boyd, 2014; fig. 9B). These foramina may have transmitted cutaneous 
branches of the inferior alveolar nerve and accompanying vessels, as in extant lepidosaurs (Oelrich, 1956; Galton, 1974a; Evans, 2008). All examined Haya specimens possess two surangular foramina, unlike a specimen of Thescelosaurus that has three on its right surangular (Oelrich, 1956; Galton, 1974a; Evans, 2008). The lateral process is a small, fingerlike projection or boss just anterior to the glenoid articulation with the quadrate. It is similarly low and rounded in Orodromeus, Zephyrosaurus, Changchunsaurus, Hypsilophodon, Tenontosaurus, and Zalmoxes (Galton, 1974a; Sues, 1980; Scheetz, 1999; Weishampel et al., 2003; Jin et al., 2010; Thomas, 2015), but is longer and more hooked in Thescelosaurus (Boyd, 2014). The surangular tapers posteriorly to form the lateral wall of a retroarticular process that expands slightly dorsoventrally distal to the glenoid. The lateral surface of the retroarticular process exhibits shallow grooves, similar to, but less pronounced than those of Thescelosaurus (Boyd, 2014), indicating probable attachment sites for the $\mathrm{m}$. depressor mandibulae or m. pterygoideus ventralis (Holliday, 2009). As in other early-diverging neornithischians, the mandibular adductor fossa excavates the medial surface of the surangular (Norman et al., 2004).

Angular: The angular is a dorsoventrally narrow, roughly triangular bone that contributes to the posterolateral and ventral portions of the mandible (fig. 3, 9). Its anterior prong slots between the dentary laterally and splenial medially. The angular also articulates with the surangular dorsally and the prearticular medially. The posterior portion of the angular is deflected ventrally, articulating with the ventrally deflected posterior portion of the surangular along most of its length. The posteriormost end comes to a sharp point where it articulates with the surangular and prearticular ventrally (fig. 4B, D). This sharp point is similar to that of Changchunsaurus, Hypsilophodon, and most other early-diverging neornthischians (Galton, 1974a; Jin et al., 2010). Due to slight disarticulation of the postdentary region, IGM 100/2014 reveals that the anterior prong articulates with the dentary and splenial along a tongue-in-groove joint (fig. 9). A ridge or crease clearly defines the transition from the convex ventral face to the flat lateral face, and is especially apparent in IGM 100/2016 (fig. 10A, C). The lateral face lacks the depression present in Thescelosaurus (Boyd, 2014).

Splenial: The splenial is a triangular, dorsoventrally expanded, mediolaterally compressed sheet of bone that articulates with the angular, dentary, and prearticular medially (figs. 4B, D, 9, 10). It covers most of the Meckelian groove. The splenial reaches its greatest dorsoventral expansion near the position of the coronoid process of the dentary, and tapers anteriorly. The angle between this dorsally expanded flange and the anterior half of the splenial is greater in Haya than in Thescelosaurus (Boyd, 2014), and both contrast with the dorsoventrally low splenial of Lesothosaurus (Porro et al., 2015). The splenial of IGM 100/2016 is lower and less triangular than that of IGM 100/2017 (though this is possibly the result of breakage). The anterodorsal border contains two or three U-shaped notches, creating a jagged outline, as in Changchunsaurus and Thescelosaurus (Jin et al., 2010; Boyd, 2014; fig. 10A). Unlike the single splenial foramen of Changchunsaurus (Jin et al., 2010), there are two small foramina on the medial surface of the left splenial in IGM 100/2017, and one or two foramina on the right splenial of IGM 100/2016 (fig. 10A). As noted by Jin et al. (2010), these foramina may correspond to the anterior mylohyoid foramen, a saurischian synapomorphy according to Rauhut (2003). However, without additional evidence, it is unclear if these foramina are homologous to the foramina of saurischians. The internal mandibular fenestra has been reduced to a small fossa between the splenial and prearticular.

Prearticular: The prearticular is a straplike, mediolaterally compressed bone forming the ventral margin of the mandibular adductor fossa (fig. 4B, D). It articulates with the angular ventrally, the dentary and splenial anteriorly, the articular posteriorly, and the surangular laterally. Its posterior end is triangular and wraps 


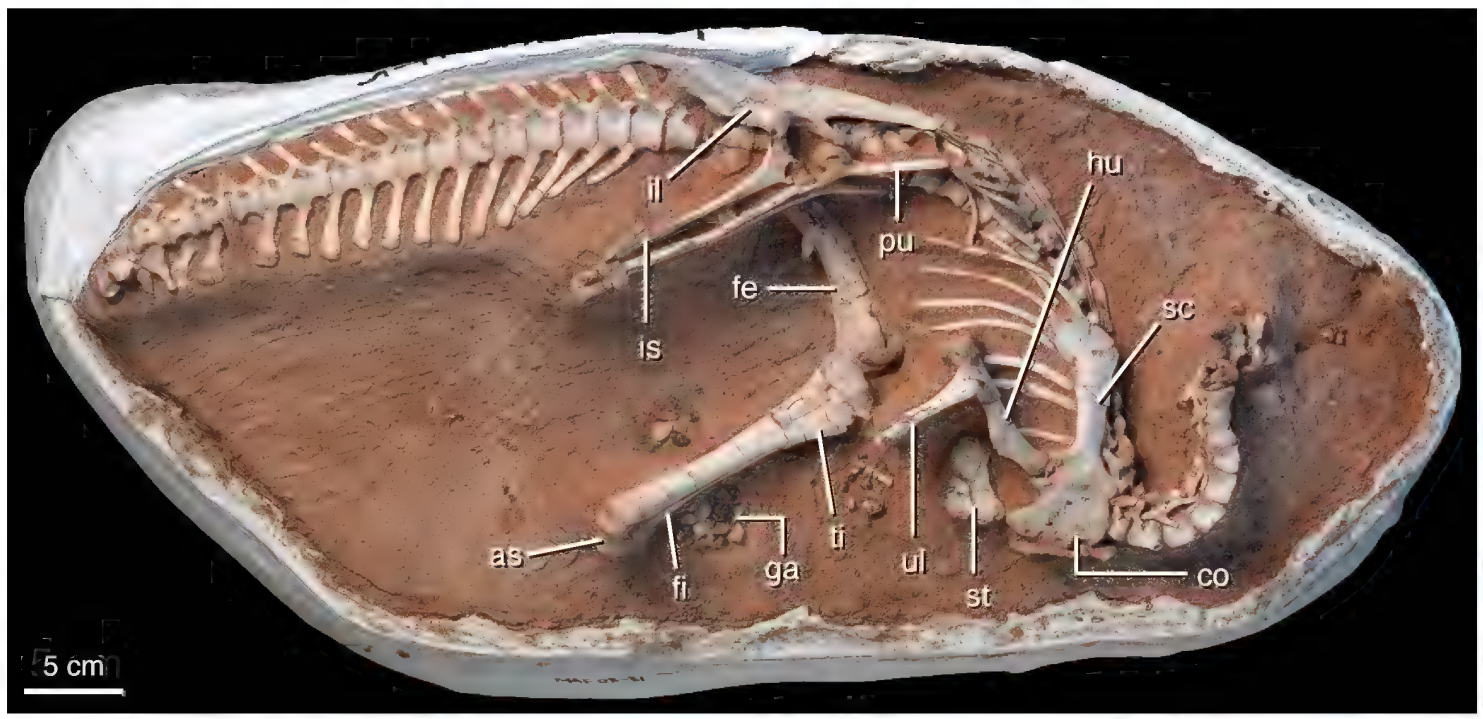

FIG. 27. Postcranial skeleton of Haya griva, IGM 100/2015. Abbreviations in appendix 1.

ventrally to meet the retroarticular process of the surangular, forming a cup for the articular. The anterior end curves anterodorsally toward the coronoid process of the dentary. An ovoid foramen lies just anterior to the suture for the articular, in the posterior end of the right prearticular of IGM 100/2017. This is possibly homologous to a similar, more anteriorly placed foramen in Hypsilophodon (Galton, 1974a). It is unclear whether this is the "slit" that Galton (1974a) considered the opening for the chorda tympani branch of c.n. VII, as the foramen for the chorda tympani is located within the articular in some pseudosuchians, Silesaurus, and some saurischian dinosaurs (e.g., Gower, 1999; Currie, 2003; Yates, 2005; Cabreira et al., 2011; Nesbitt, 2011; Sereno et al., 2012). There is also a foramen in the articular of a specimen of Psittacosaurus mongoliensis, IGM 100/1930 (D.E.B., personal obs.). In birds, the chorda tympani does not enter the articular, instead traveling medially outside the bone (Witmer, 1990). The lack of foramina in the articular in some ornithischians suggests that the chorda tympani either followed a similar path to that in birds, entered a space between the articular and prearticular, or entered the prearticular itself. There is a fora- men between the articular and prearticular in Triceratops and Centrosaurus (= Monoclonius), as well as an additional foramen within the prearticular in Centrosaurus (Brown and Schlaikjer, 1940). A specimen of Tenontosaurus also appears to have a foramen in its prearticular (Thomas, 2015: fig. 57). This, combined with the evidence from Psittacosaurus noted above, suggests that there is a diversity of probable paths for the chorda tympani within ornithischians.

ARTICULAR: The articular is a thick, roughly pyramidal bone situated between the prearticular medially, the surangular laterally, and the angular ventrally (figs. 3, 4B). It does not wrap ventrally around the prearticular, as does the articular of Hypsilophodon (Galton, 1974a). The dorsal surface contains the saddle shaped glenoid for articulation with the medial distal condyle of the quadrate. The posterior face of the articular exhibits a concavity near its dorsolateral margin. No foramina pierce the articular (see the Prearticular section above for discussion of the implications this holds for the path of the chorda tympani branch of c.n. VII). The articular is relatively shorter anteroposteriorly than that of Thescelosaurus (Boyd, 2014). 


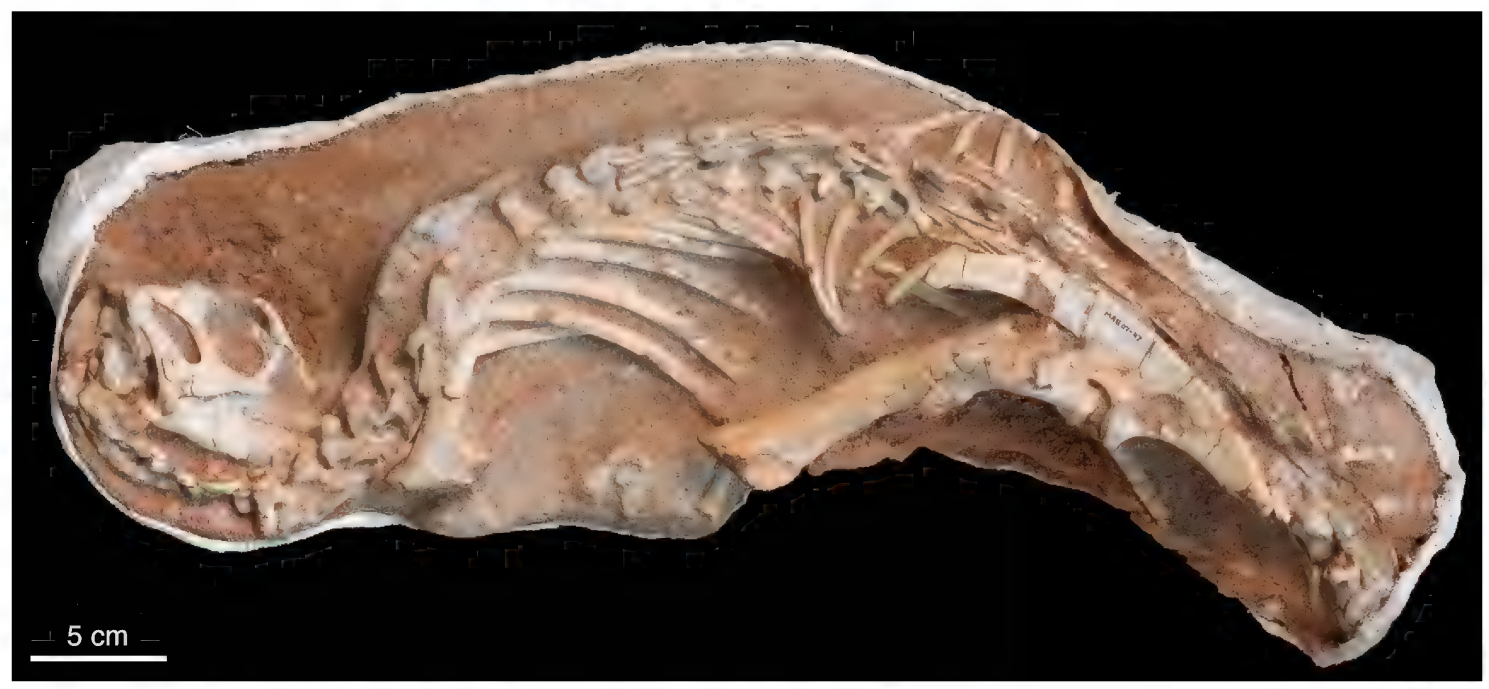

FIGURE 28. Articulated skull and skeleton of Haya griva, IGM 100/2019.

Coronoid: No coronoids are clearly visible in any of the specimens. What can be observed on IGM 100/2017 suggests that the coronoid forms a dorsally directed prominence that articulates with the dentary medial to its coronoid process, as in Hypsilophodon (Galton, 1974a).

\section{Axial Skeleton}

The following description is based primarily on IGM 100/2015 (fig. 27), IGM 100/2019 (fig. 28), IGM 100/3137 (fig. 29), and IGM 100/3178 (figs. 32, 36, 39, 40, 45, 46, 48), supplemented with other specimens where noted. The axial skeleton of Haya griva consists of nine cervical, 15 dorsal, six sacral, and $22+$ caudal vertebrae (Makovicky et al., 2011).

Cervical Vertebrae and Cervical Ribs: Primitively, early-diverging neornithischians possess nine cervical vertebrae (Norman et al., 2004), the same as in Haya griva. The atlas is comprised of the two unfused halves of the neural arch (the neurapophyses), an unfused atlantal intercentrum, and a pleurocentrum fused to the axis to become the odontoid process of the axis. No unambiguous trace of a proatlas is preserved in any of the specimens, though this element is present in Agilisaurus, Hexinlusaurus,
Orodromeus, and Hypsilophodon (Norman et al., 2004). Each neurapophysis is hook shaped and articulates tightly with the lateral portion of the occipital condyle along an anteriorly facing fossa. The neurapophyses articulate dorsally with each other above the neural canal and with the atlantal intercentrum ventrally along straight sutures (fig. 31). The atlantal intercentrum of IGM $100 / 2015$ is currently missing because of an unknown disturbance to the specimen that apparently disarticulated the bone before photographs were taken for the current work. Efforts to locate the atlantal intercentrum are ongoing as of January 2021. We wrote the following description from our firsthand observations of the original element. Its morphology is also recorded by a cast (AMNH FARB 30635) and Makovicky et al. (2011: fig. 3A, B). The atlantal intercentrum is concave posteriorly and roughly crescentic in ventral view (fig. $31 \mathrm{~B}$ ). It is somewhat wedge shaped in lateral view, with a convex, anteroventrally sloping anterior surface (fig. 31C). A shallow depression on the anterodorsal surface is contiguous with the anterior surfaces of the neurapophyses. It is probably an articular facet for the occipital condyle. The posterior surface of the atlantal intercentrum exhibits an elliptical groove into which 


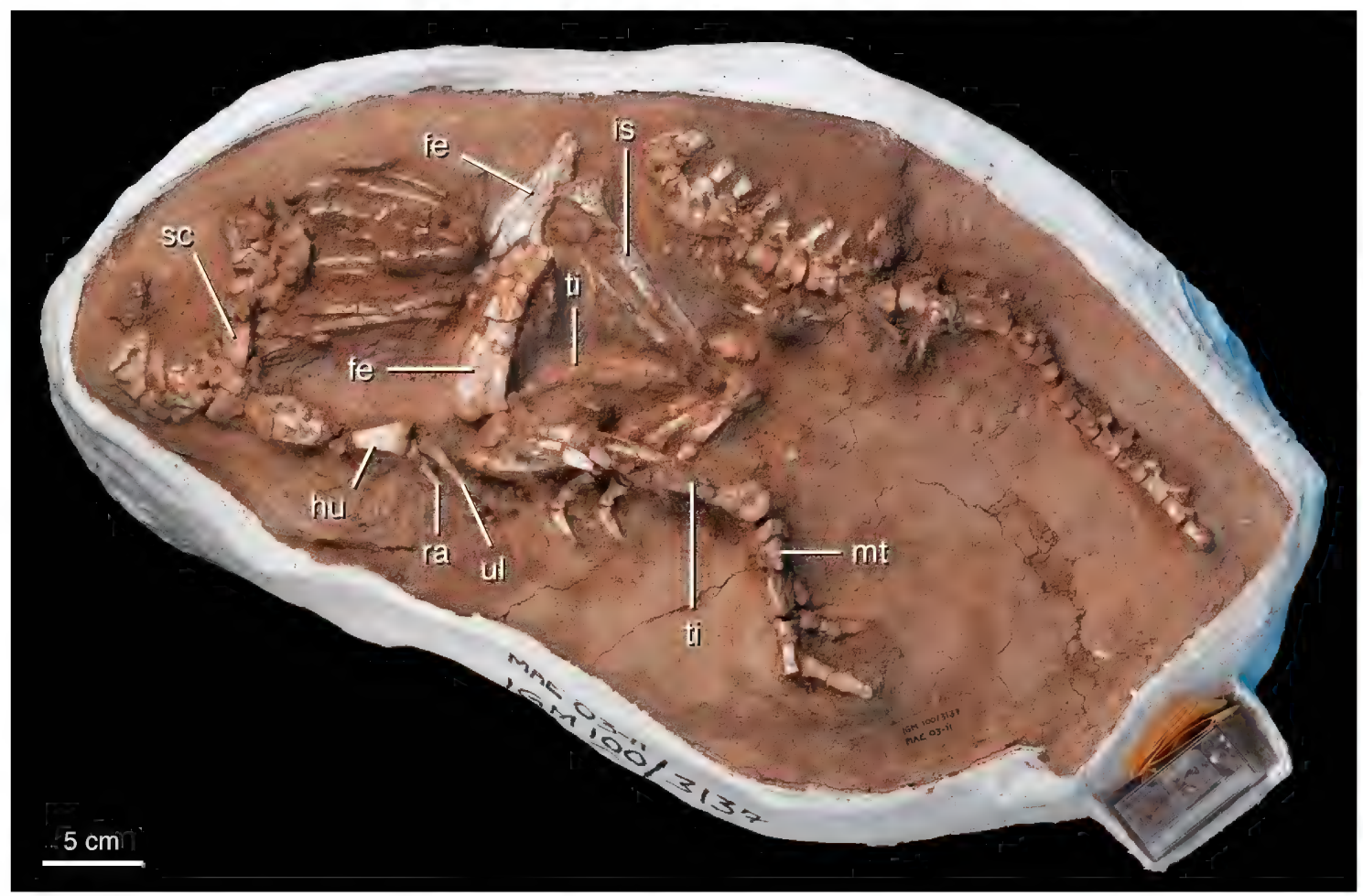

FIG. 29. Postcranial skeleton of Haya griva, IGM 100/3137. Abbreviations in appendix 1.

the axial intercentrum inserted. The diapophysis for the first cervical rib is present on the lateral surface of the atlantal intercentrum. In IGM 100/2017 and 100/3178 (figs. 30, 32) this simple, fairly straight rib is mediolaterally flattened anteriorly and tapers posteriorly, extending to a point beneath the third cervical. The odontoid process consists of a blocky base articulating with the axial centrum. From this emerges an anteriorly tapering rounded process with a flat to slightly concave dorsal surface. The odontoid process is clearly unfused to the axis in IGM $100 / 2015$ and may have shifted postmortem, so that it appears to articulate posteriorly with the axial neural spine and centrum near the suture between those two elements. This contrasts with IGM 100/2017 where the articulation is primarily with the axial centrum. Overall, the morphology and arrangement of the constituent elements of the atlas is very similar to that of Hypsilophodon (Galton, 1974a), except for the lack of an obvious proatlas in Haya.
The axis complex consists of the centrum, neural arch, and axial intercentrum. The neural arch is not fused to the centrum in IGM $100 / 2015$. The neural spine of the axis is more dorsoventrally expanded and is directed slightly more anteriorly in Haya than in Jeholosaurus and Changchunsaurus (Butler et al., 2011; Han et al., 2012). The dorsal border of the axial neural spine in IGM 100/2014 and IGM 100/3178 is straight or weakly convex in lateral view, as in Changchunsaurus and Hypsilophodon (Galton, 1974a; Butler et al., 2011). This contrasts with the gently concave dorsal border of IGM 100/2019, which is similar in this respect to Heterodontosaurus (Santa Luca, 1980; Galton, 2014), but not as extreme as in Lesothosaurus (Baron et al., 2017). The dorsal border of the axial neural spine of IGM 100/2015 is gently sigmoidal in lateral view, transitioning from slightly concave anteriorly to convex posteriorly (fig. 31). In both IGM 100/2019 (fig. 18B) and Jeholosaurus this border has a scalloped natural edge (Han et al., 2012). 


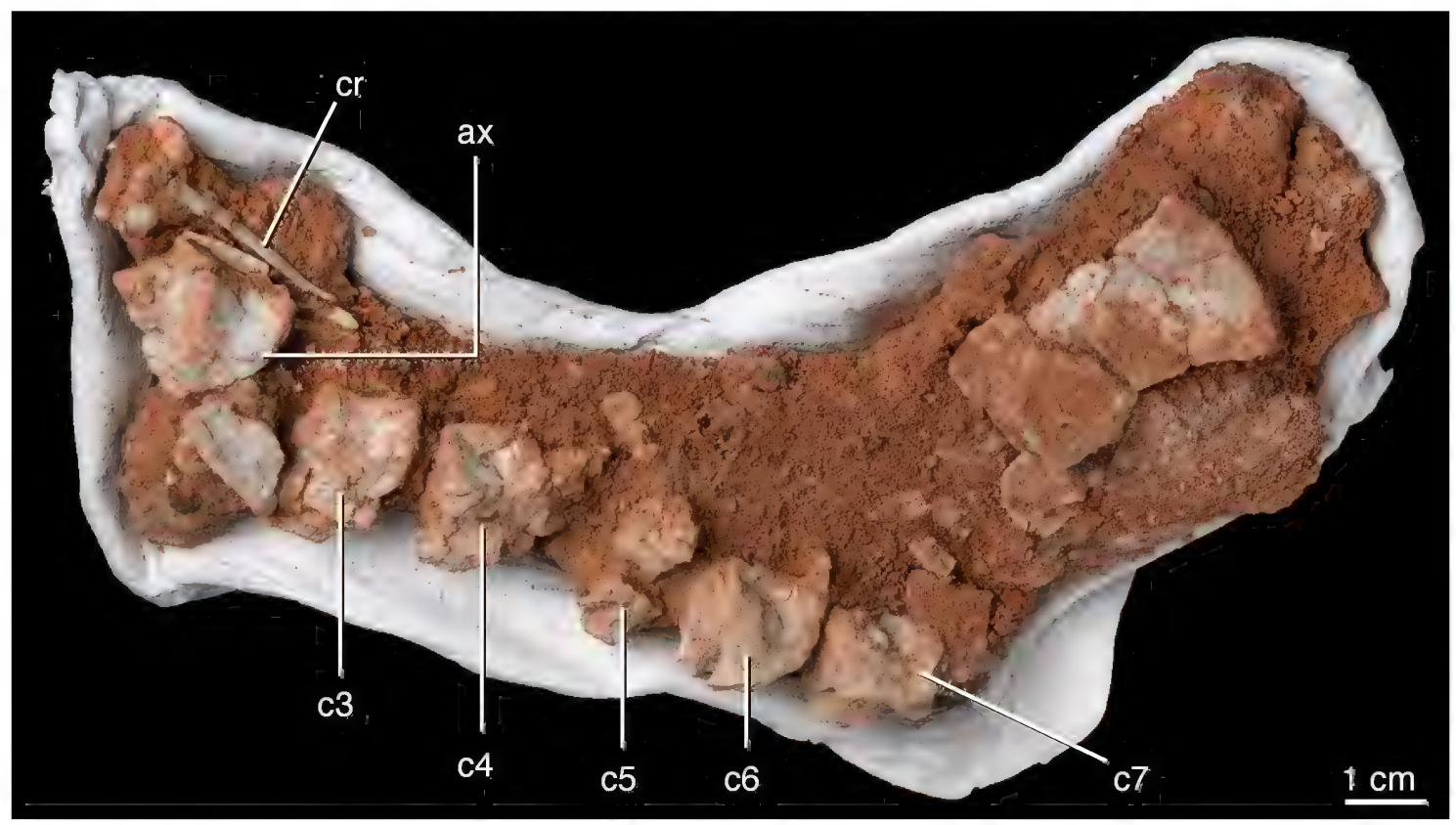

FIG. 30. Cervical vertebrae of the holotype of Haya griva, IGM 100/2017. Abbreviations in appendix 1.

The apex of the axial neural spine is more laterally expanded than in Heterodontosaurus (Galton, 2014). Short postzygapophyses that lack epipophyses are present on the posterior side of the neural spine near its base (Makovicky et al., 2011). The axial intercentrum is semicircular in ventral view and articulates with the centrum of the axis anteroventrally, projecting just anterior to the anterior border of the centrum (fig. 31). It is smaller than the atlantal intercentrum, with which it articulates via a tongue-and-groove articulation. The keel on the ventral surface of the centrum is broader and flatter than the keels of the postaxial cervical vertebrae. The axial centrum of Jeholosaurus exhibits a sharper keel (Han et al., 2012). The lateral depressions on the Haya axial centrum are not as deep as those on cervicals three through nine. The neurocentral suture bisects the parapophysis. The axis of Haya possesses well-developed parapophyses and small diapophyses (Makovicky et al., 2011). Due to the lack of well-preserved axial ribs in life position, it is not clear whether the axial rib of Haya was single or double headed or whether it articulated with the parapophysis as in Changchunsaurus (Butler et al., 2011), or with the diapophysis as in Jeholosaurus and Hypsilophodon (Galton, 1974a; Han et al., 2012).

Cervical vertebrae three through nine are approximately as long as tall, as in Jeholosaurus, Yueosaurus, Orodromeus, Changchunsaurus, and Thescelosaurus (Galton, 1974b; Scheetz, 1999; Butler et al., 2011; Han et al., 2012; Zheng et al., 2012), but in contrast to the anteroposteriorly elongate cervical centra of Koreanosaurus and elasmarians such as Talenkauen and Macrogryphosaurus (Novas et al., 2004; Calvo et al., 2007; Huh et al., 2011). The third cervical vertebra differs from successive cervicals by a sharply concave ventral border of its centrum, giving the centrum a bent or kinked appearance in lateral view (figs. 30, 31), similar to the condition in Orodromeus (Scheetz, 1999). Successive cervical vertebrae have roughly quadrangular to trapezoidal centra, with slightly shorter dorsal than ventral borders present on cervicals six through nine, allowing the neck to maintain a gentle curve near its base. Their lateral surfaces contain 


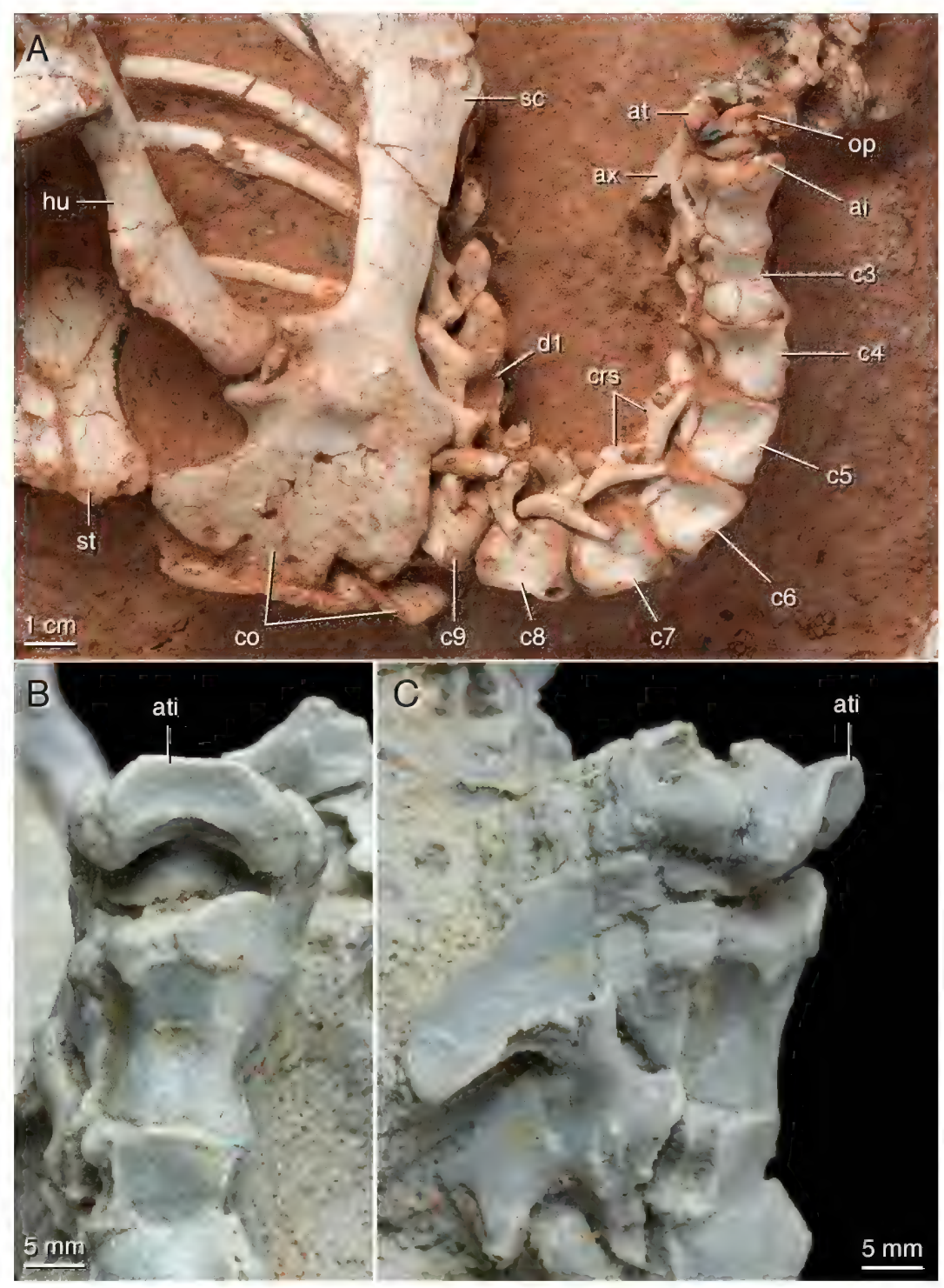

FIG. 31. A, Cervical vertebrae of IGM 100/2015 in right ventrolateral view. B, Atlantal intercentrum of cast of IGM 100/2015 (AMNH FARB 30635) in ventral view. C, Atlantal intercentrum of cast of IGM 100/2015 (AMNH FARB 30635) in lateral view. Abbreviations in appendix 1.

broad, shallow depressions, with anterior lips that extend further laterally than the posterior borders of the centra. In contrast to the condition in Changchunsaurus (Butler et al., 2011), no nutrient foramina are present on the lateral surfaces of the centra. Though the articulated nature of IGM 100/2015 and IGM 100/2019 obscures most of the articular facets of the cervical centra,
IGM 100/2017 reveals that most are probably amphiplatyan (fig. 30). The ventral borders of the centra are straight to slightly convex; there is no clear pattern to these slight variations in shape between successive vertebrae. All postaxial cervical vertebrae are ventrally keeled, with the sharpness of the keel diminishing slightly posteriorly, starting with the sixth cervical vertebra. The par- 


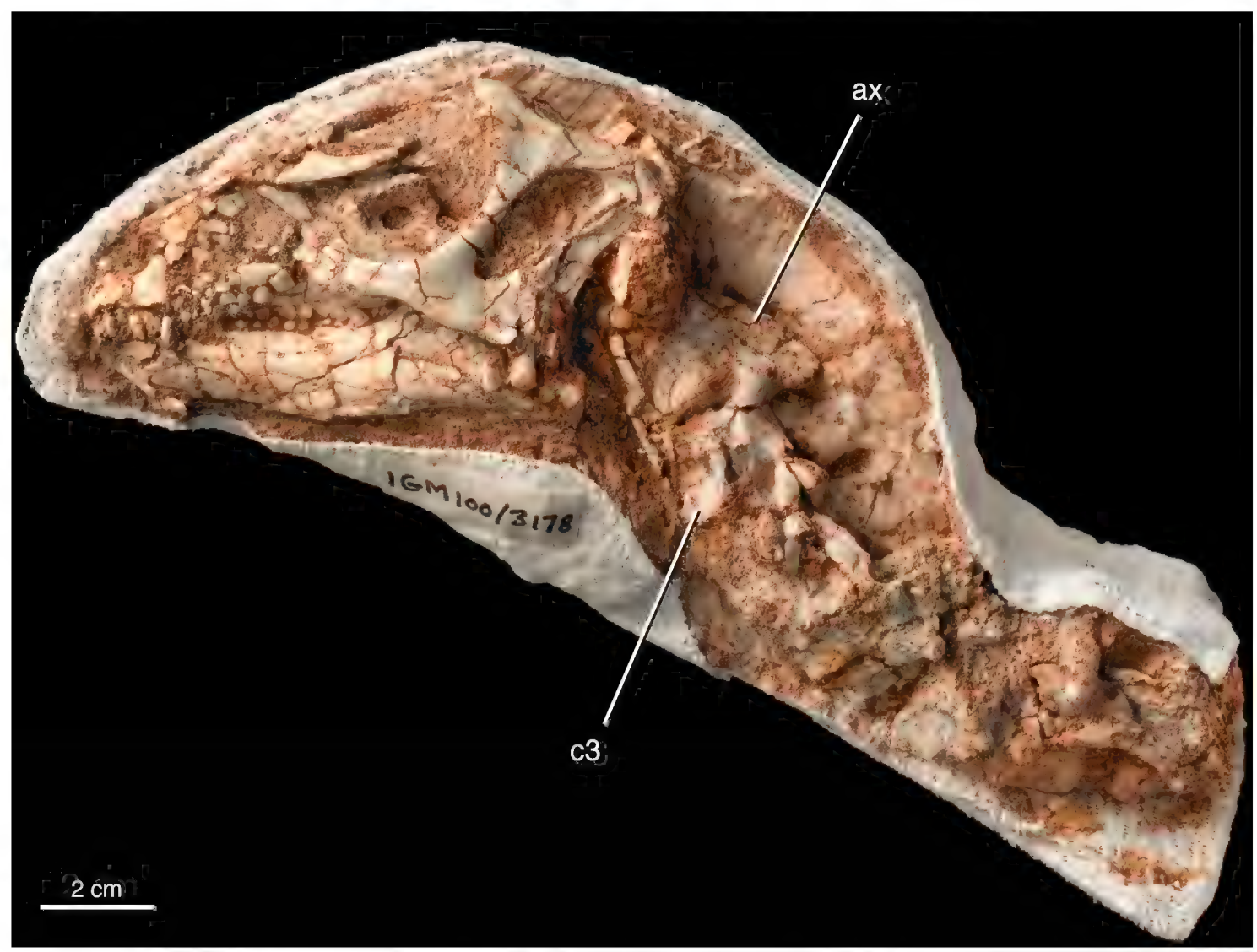

FIG. 32. Skull and cervical vertebrae of Haya griva, IGM 100/3178, in left lateral view. Abbreviations in appendix 1 .

apophyses extend across and are divided by the neurocentral sutures in cervicals four through nine of IGM 100/2015, similar to the condition in Jeholosaurus (Han et al., 2012). The extent of the parapophysis on the third cervical vertebra of IGM 100/2015 is harder to discern, given partial disarticulation of the neural arch from the centrum. Parapophyses are directed anterolaterally to laterally in cervicals three through six, becoming more posterolaterally directed in cervicals seven through nine. Where visible, all neural arches appear unfused to their centra in IGM $100 / 2015$ and IGM 100/2017 (figs. 30, 31); this is not easily assessed in the other specimens due to overlying bones or matrix. Cervical neural spines are largely obscured or eroded in IGM 100/2015, IGM 100/2019, and IGM 100/3178, but all were likely shorter and more laterally compressed than the axial neural spine. Jeholosaurus, Changchunsaurus, and Haya share a third cervical with a relatively tall neural arch and an anteriorly expanded spine that projects just dorsal to the postzygapophyses (Butler et al., 2011; Makovicky et al., 2011; Han et al., 2012). The neural spine of the ninth cervical is taller and anteroposteriorly narrower than that of the eighth, creating a transition in neural spine height to the taller spine of the first dorsal vertebra (figs. 31, 33). Cervical neural canals are broad and semicircular. The prezygapophyses of at least the third through fifth cervicals are directed anteroventrally, as in most other noncerapodan neornithischians. Postzygapophyses are anteroposteriorly short and located high on the neural arch, just ventral 


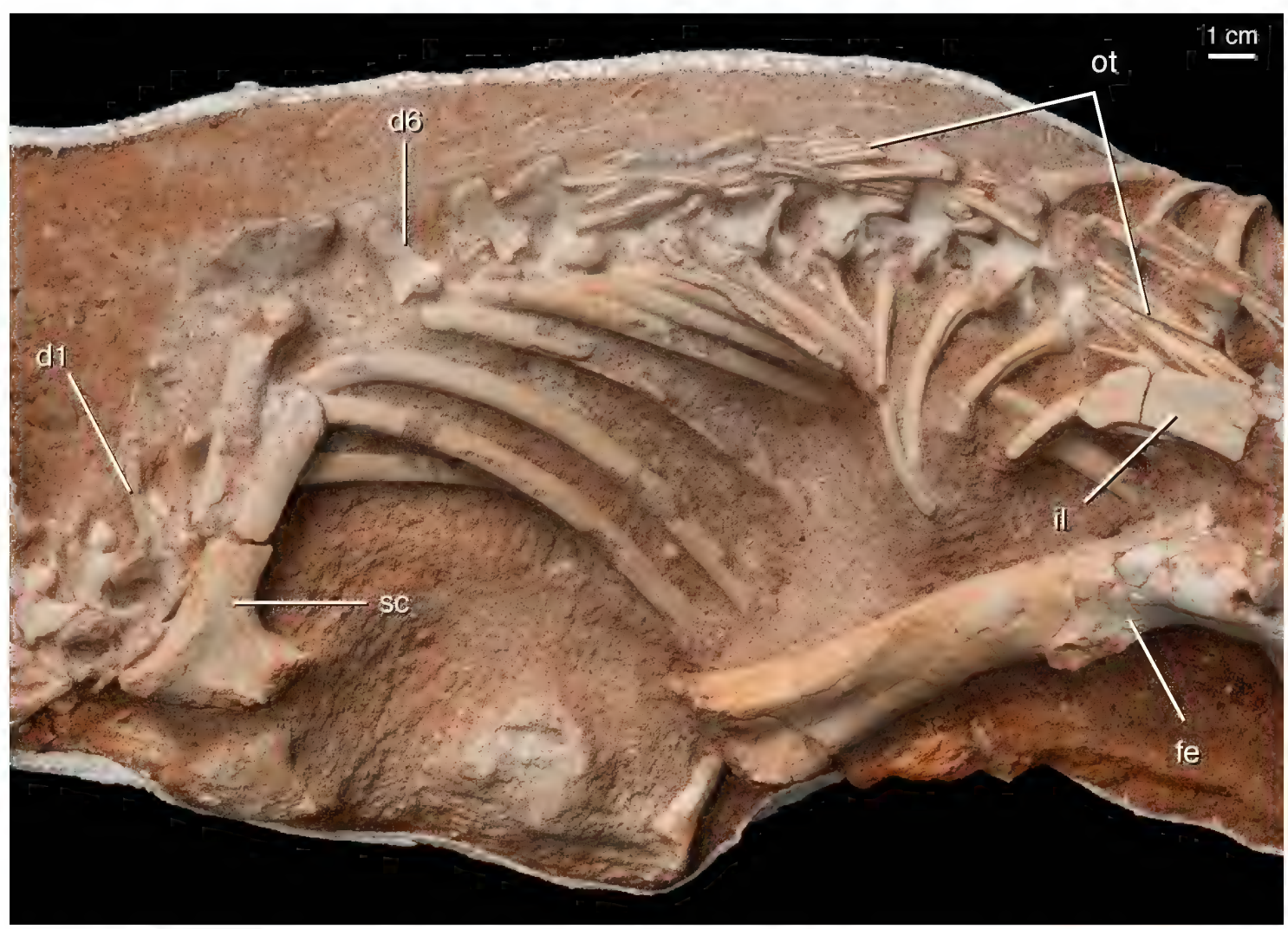

FIG. 33. Dorsal vertebrae of IGM 100/2019 in left dorsolateral view. Abbreviations in appendix 1.

to the neural spine. Both pre- and postzygapophyseal articular surfaces are flat.

Postaxial cervical ribs are double headed, with a shorter, rounded tuberculum, and a longer, narrower capitulum, as in Changchunsaurus, Orodromeus, and Hypsilophodon (Galton, 1974a; Scheetz, 1999; Butler et al., 2011). In contrast, the relative proportions of the tuberculum and capitulum are more nearly equal in Hexinlusaurus and Jeholosaurus (He and Cai, 1984; Han et al., 2012). Keels are present on the lateral surfaces of the capitulae of cervical ribs five, six, and seven near their junctions with the turberculae, similar to the ridges in Heterodontosaurus, Changchunsaurus, and Hypsilophodon, and the iguanodontians Camptosaurus and Iguanodon (Galton, 1974a; Norman, 1980; Santa Luca, 1980; Carpenter and Wilson, 2008; Butler et al., 2011; Galton, 2014). In Haya, these ridges do not parallel the long axis of the capitulum. Instead, they angle dorsally at an angle of approximately $20^{\circ}$ to the long axis of the capitulum. The angle between the capitulum and tuberculum is more acute than in Hypsilophodon (Galton, 1974a). The distal ends of the cervical ribs are broken or obscured in all Haya specimens, preventing assessment of their original lengths.

Dorsal Vertebrae and Dorsal Ribs: The first dorsal vertebra is differentiated from the last cervical by a lateral migration of the diapophysis onto a short, rectangular transverse process connected by thin laminae to the prezygapophysis and centrum. These differences are clearly visible in IGM 100/2019 (fig. 33). Dorsal centra are completely obscured in IGM $100 / 2019$, and only the posterior dorsal centra are exposed in IGM 100/2015. The posterior dorsal centra of IGM 100/2015 are approxi- 


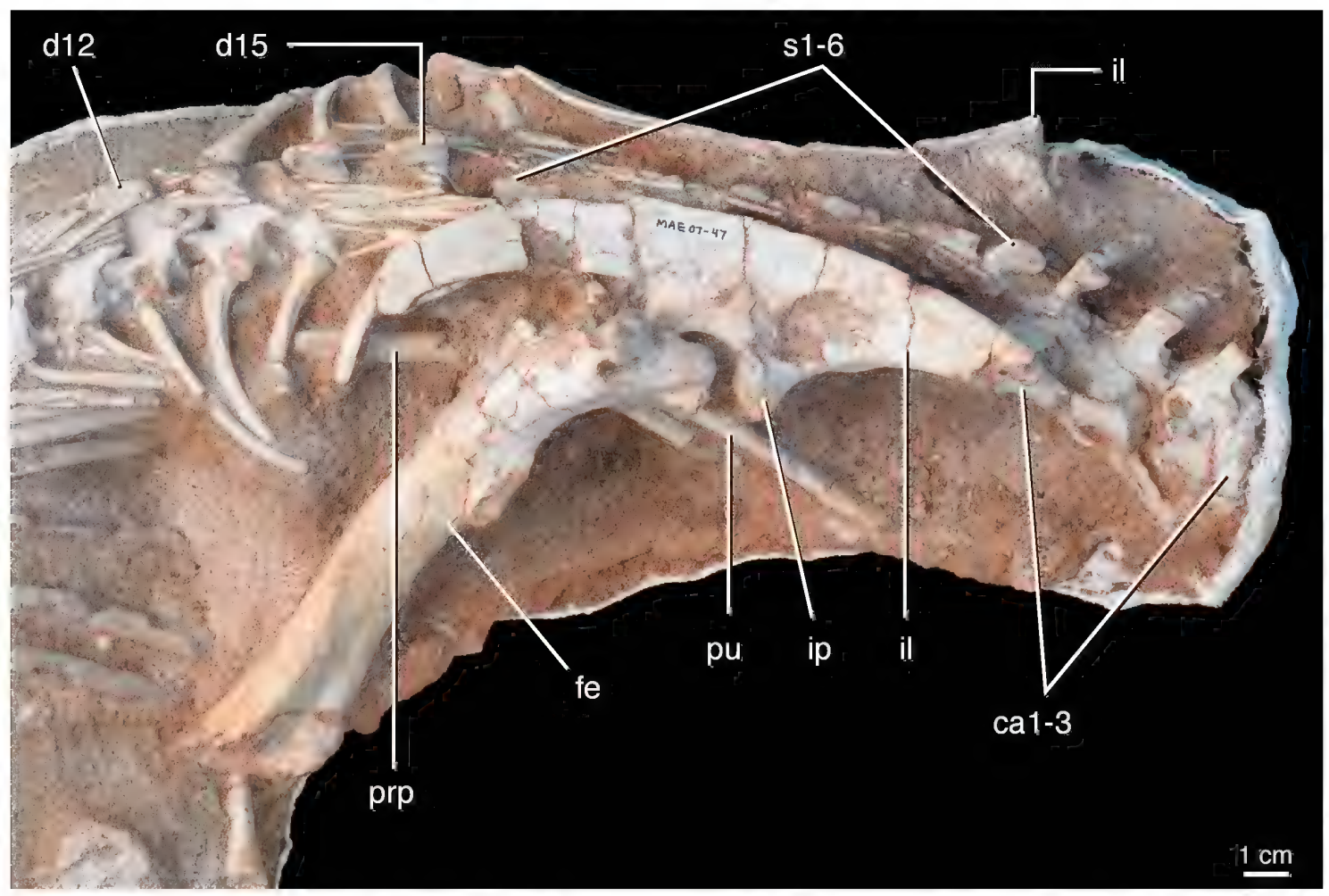

FIG. 34. Sacral region of IGM 100/2019 in left dorsolateral view. Abbreviations in appendix 1.

mately as long anteroposteriorly as they are tall dorsoventrally, and have only faint ventral keels (fig. 35). More observations can be gleaned from the dorsals of the two specimens from Zos Canyon, IGM 100/3661 and IGM 100/3181. The latter specimen includes vertebrae from the middle to posterior part of the dorsal series, probably dorsals eight through 15 , based on the morphology of the transverse processes and corresponding ribs. These vertebrae are amphicoelous, with slight ventral keels (Norell and Barta, 2016), as in Jeholosaurus (Han et al., 2012). The posterior dorsal centra of IGM 100/3181 are anteroposteriorly longer relative to their dorsoventral height than those of IGM 100/2015. Isolated dorsals of uncertain serial position from IGM 100/3661 (fig. 14) have flat to gently concave articular facets. The ventral borders of these centra are concave in lateral view. The posterior dorsals of IGM 100/2015 have striated rims around their anterolateral and posterolateral borders, as in many neornithischian taxa including Hexinlusaurus, Jeholosaurus, Yueosaurus, Orodromeus, Changchunsaurus, Parksosaurus, Thescelosaurus and Hypsilophodon (Gilmore, 1915; Galton, 1974a; He and Cai, 1984; Scheetz, 1999; Brown et al., 2011; Butler et al., 2011; Han et al., 2012; Zheng et al., 2012). These striated surfaces probably mark attachment sites for the hypaxial muscles (Butler et al., 2011). No foramina are visible on the right lateral surfaces of these centra, in contrast to their presence in Jeholosaurus and Changchunsaurus (Butler et al., 2011; Han et al., 2012). The presence of a "lumplike" swelling on the ventral surface of the first four dorsals, as in Jeholosaurus and Changchunsaurus (Butler et al., 2011; Han et al., 2012), cannot be assessed in the observed Haya specimens due to obscuring matrix. As noted by Makovicky et al. (2011), the transverse processes are angled somewhat dorsally on the anterior dorsals, transitioning to nearly horizontal near the midpoint of the dor- 


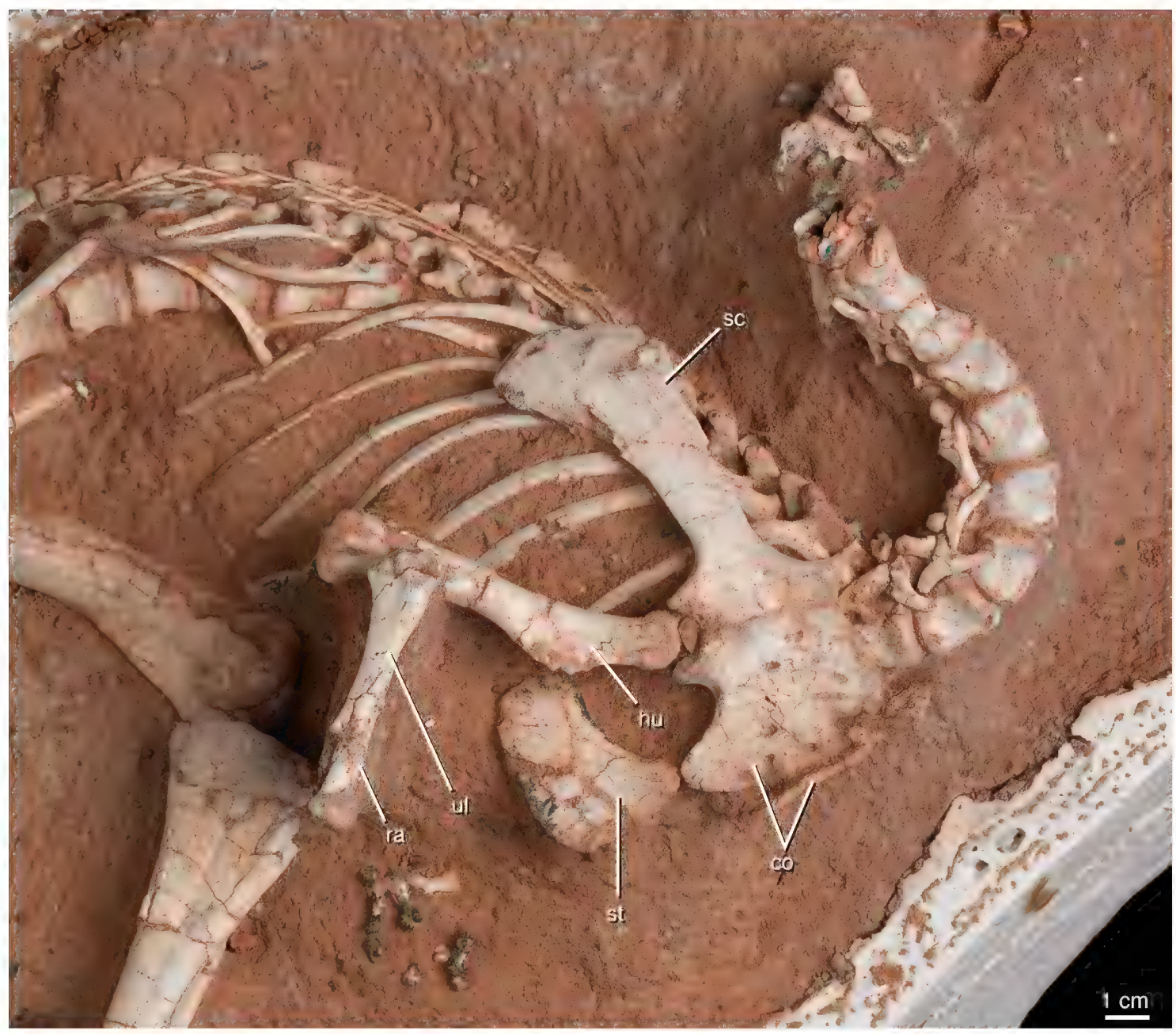

FIG. 35. Right pectoral girdle and forelimb of IGM 100/2015. Abbreviations in appendix 1.

sal series. The ventral surfaces of the transverse processes of IGM 100/3181 contain a shallow fossa, as in Changchunsaurus (Butler et al., 2011), but in contrast to Jeholosaurus, in which this fossa is divided into anterior and posterior fossae by a ridge (Han et al., 2012). Parapophyses are likely located on the neural arch ventral to the diapophysis in the anterior dorsals, but these are obscured in articulated specimens. By at least the 10th dorsal, the parapophyses have migrated dorsally to the anteroventral surfaces of the transverse processes. From the 10th to the 13th dorsals, the parapophyses show a serial reduction in size and migrate posterodorsally to touch the diapophysis. They merge to form a single articular facet on the 14th and 15th dorsals, corresponding to the single-headed ribs of those vertebrae, a feature shared with Orodromeus and Hypsilophodon (Galton, 1974a; Scheetz, 1999). This contrasts with Changchunsaurus, in which the transition to a single articular facet is made one serial position earlier, on the 13th dorsal (Butler et al., 2011), with Jeholosaurus, in which a single facet is present on dorsals 12-15 (Han et al., 2012), and with Heterodontosaurus, in which only the last dorsal has a single facet (Santa Luca, 1980; Galton, 2014). Neural spines of anterior dorsal vertebrae 


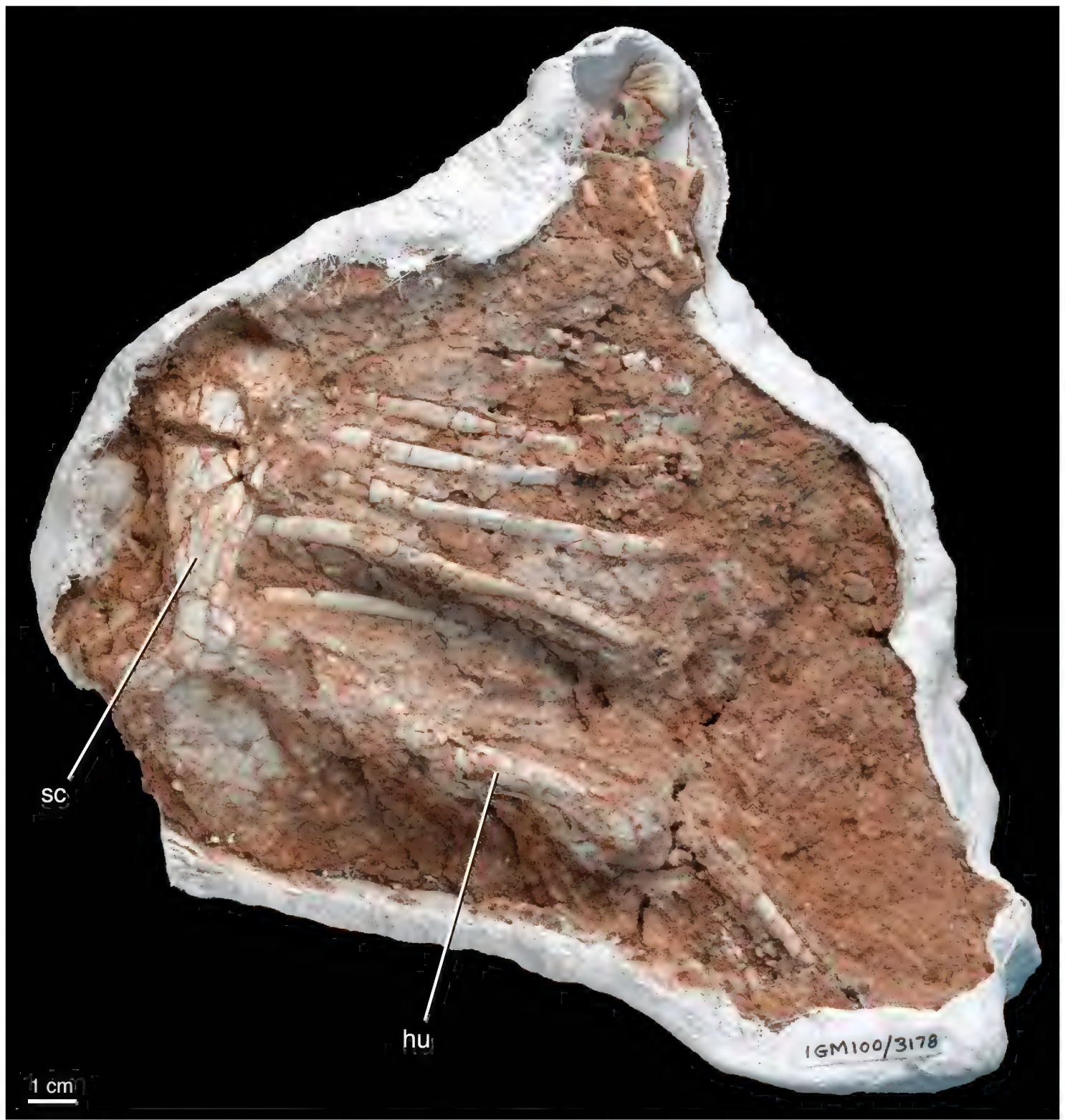

FIG 36. Left pectoral girdle and forelimb of IGM 100/3178. Abbreviations in appendix 1.

are narrow and posterodorsally directed. Beginning around the fifth dorsal, neural spines are vertical and are anteroposteriorly expanded, having rectangular outlines in lateral view. In lateral view, the prezygapophyses point anterodorsally at an angle of about $45^{\circ}$ from horizontal, whereas the postzygapophyses point straight horizontally. Articular facets of the pre- and postzygapophyses are flat. As in Jeholosaurus, the plane between the dorsomedially facing prezygapophyseal facets and the ventrolaterally facing postzygapophyseal facets is oriented at about $45^{\circ}$ to horizontal (Han et al., 2012). The 10th and 11th dorsals of IGM 100/2015 have neural arches unfused to their respective centra. The state of fusion cannot be determined for the 


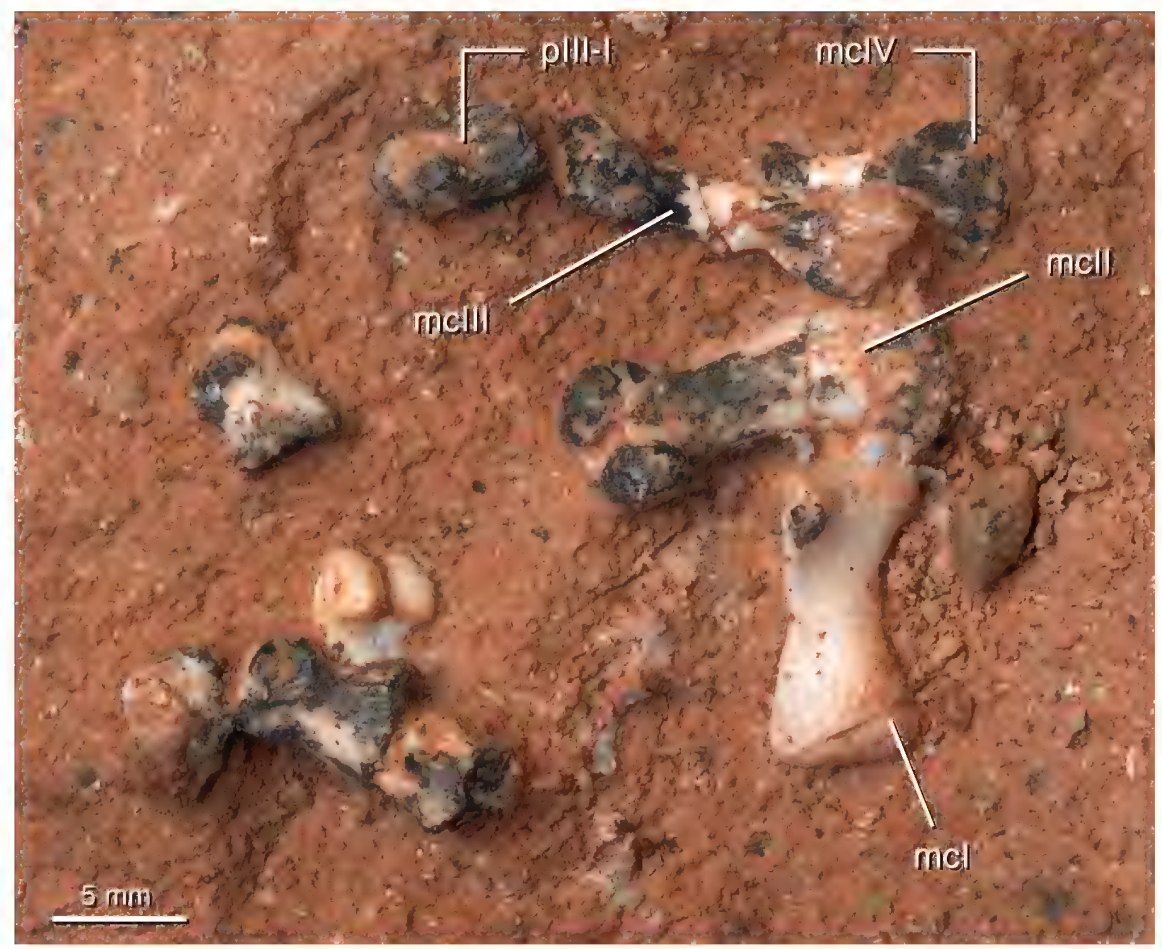

FIG. 37. Manus elements of IGM 100/2015. Abbreviations in appendix 1.

articulated specimens due to obscuring matrix, nor for IGM 100/3181 due to weathering of its vertebrae. Two isolated dorsal neural arches of IGM 100/3661 lack their centra, indicating these elements were unfused. The neural canal in the probable posterior dorsal of IGM 100/3661 is ovoid, being slightly taller dorsoventrally.

Though the distal ends of some ribs are obscured in the specimens examined, successive ribs appear to increase in length posteriorly until the fourth or fifth rib, after which they decrease gradually in length and curvature (fig. 33). Changchunsaurus and Koreanosaurus have a similar pattern of changes in rib lengths (Butler et al., 2011; Huh et al., 2011). As described by Makovicky et al. (2011), the cross section of ribs 1-11 changes from elliptical proximally to mediolaterally flattened distally. The capitulum decreases in length posteriorly, eventually merging with the tuberculum to form single-headed 14 th and 15th ribs, as noted above. Dorsal ribs 12 through 14 have grooves on the proximal portion of their posterior surfaces (figs. 33, 34). Makovicky et al. (2011) described the posterior surface of these ribs as having a ridge, but this is better characterized as a single groove bounded by two ridges. The groove on the 12 th rib is shallower than on the next two in the sequence. No ossified sternal ribs are preserved (Makovicky et al., 2011). Cartilaginous sternal ribs almost certainly were present, given that sternal ribs are probably plesiomorphic for at least Amniota (Parker, 1868), and that ossified sternal ribs have been found in other early-diverging neornithischians, including Othnielosaurus, Thescelosaurus, Parksosaurus, and Hypsilophodon (Gilmore, 1915; Parks, 1926; Galton and Jensen, 1973; Galton, 1974a). Their lack of ossification likely reflects either immaturity or the retention of cartilaginous sternal ribs throughout ontogeny, in contrast to the other aforementioned neornithischians in which sternal ribs ossify. Similarly, no ossified intercostal plates are present, in contrast to Othnielosaurus, Parkso- 


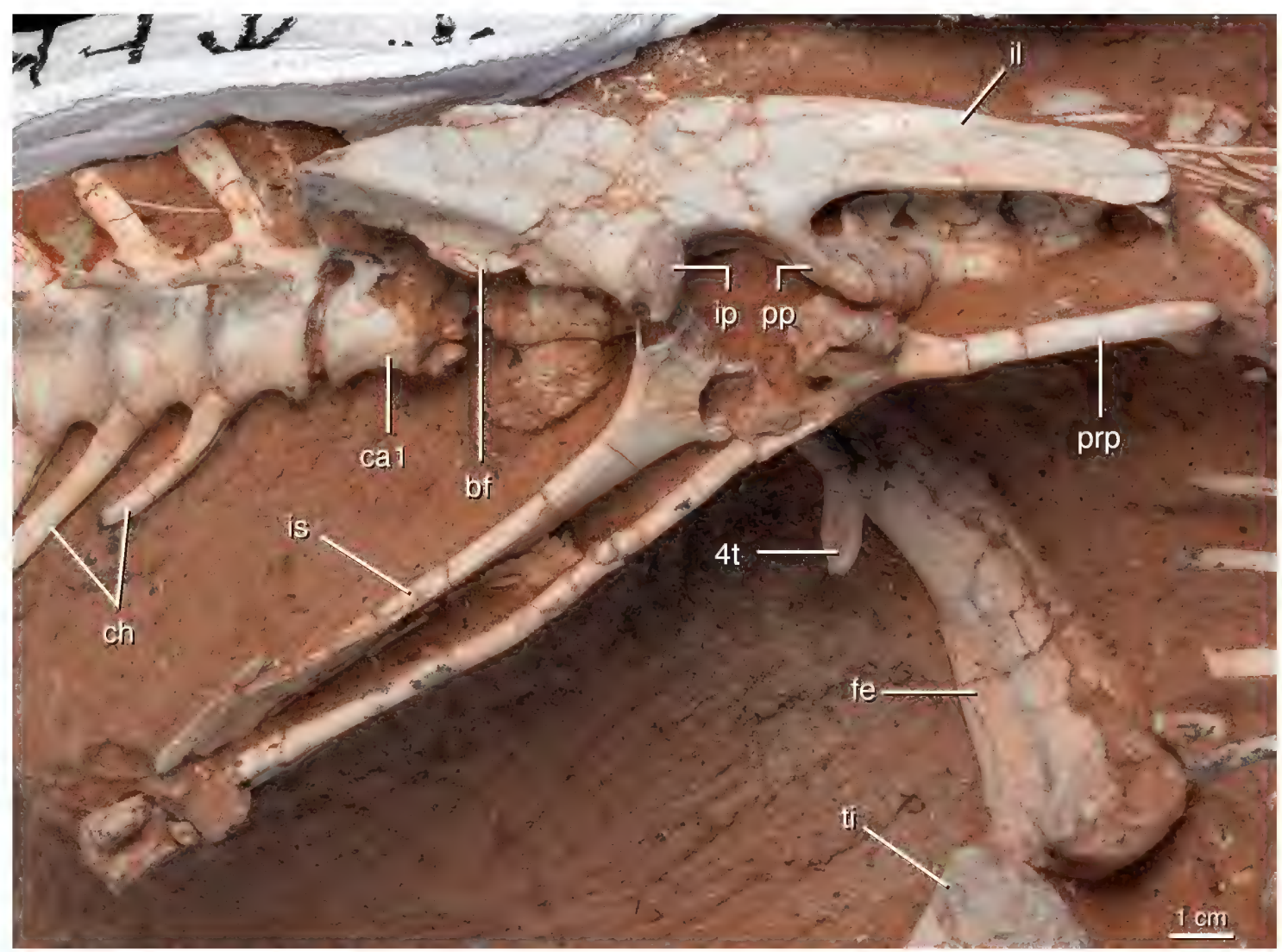

FIG. 38. Pelvis of IGM 100/2015 in right lateral view. Abbreviations in appendix 1.

saurus, Thescelosaurus, Talenkauen, Macrogryphosaurus, and Hypsilophodon (Calvo et al., 2007; Butler and Galton, 2008). The absence of intercostal plates either may be a true phylogenetic difference between Haya and those taxa, or it may simply reflect the immature nature of the Haya specimens. Gastralia are absent, as in all ornithischians (Claessens, 2004).

Sacral Vertebrae and Sacral Ribs: Two of the Haya specimens studied, IGM 100/2015 and IGM 100/2019 (figs. 27, 34), have complete sacral series that allow for a full count of the vertebrae, and form the basis for most of the description below. IGM 100/3178 has sacral vertebrae, but they are almost completely obscured by matrix (fig. 39). IGM 100/3182 preserves two sacral vertebrae exposed in left lateral view (fig. 50). Both IGM 100/2015 and IGM 100/2019 have six sacrals (figs. 34,38 ). The first and sec- ond sacral vertebrae of IGM 100/2015 are dorsosacrals with short, anterolaterally directed ribs that articulate with the ilium (fig. 38). Dorsosacrals are dorsal vertebrae that have fused to the successive plesiomorphic posterior sacrals (e.g., Griffin et al., 2017). The addition of these vertebrae increases the number of sacrals to six in Haya, as is typical for noncerapodan neornithischians other than Agilisaurus, Hexinlusaurus, and possibly Thescelosaurus assiniboiensis, which have or may have five sacrals (Norman et al., 2004; Brown et al., 2011), and Oryctodromeus and possibly Orodromeus, which have or may have seven (Varricchio et al., 2007). A specimen of Thescelosaurus sp., AMNH FARB 117 (Galton, 1974b), also has seven sacral vertebrae, as its first two sacrals are dorsosacrals with ribs that articulate with the preacetabular portion of the ilium. We disagree with Galton's (1974b) interpretation 


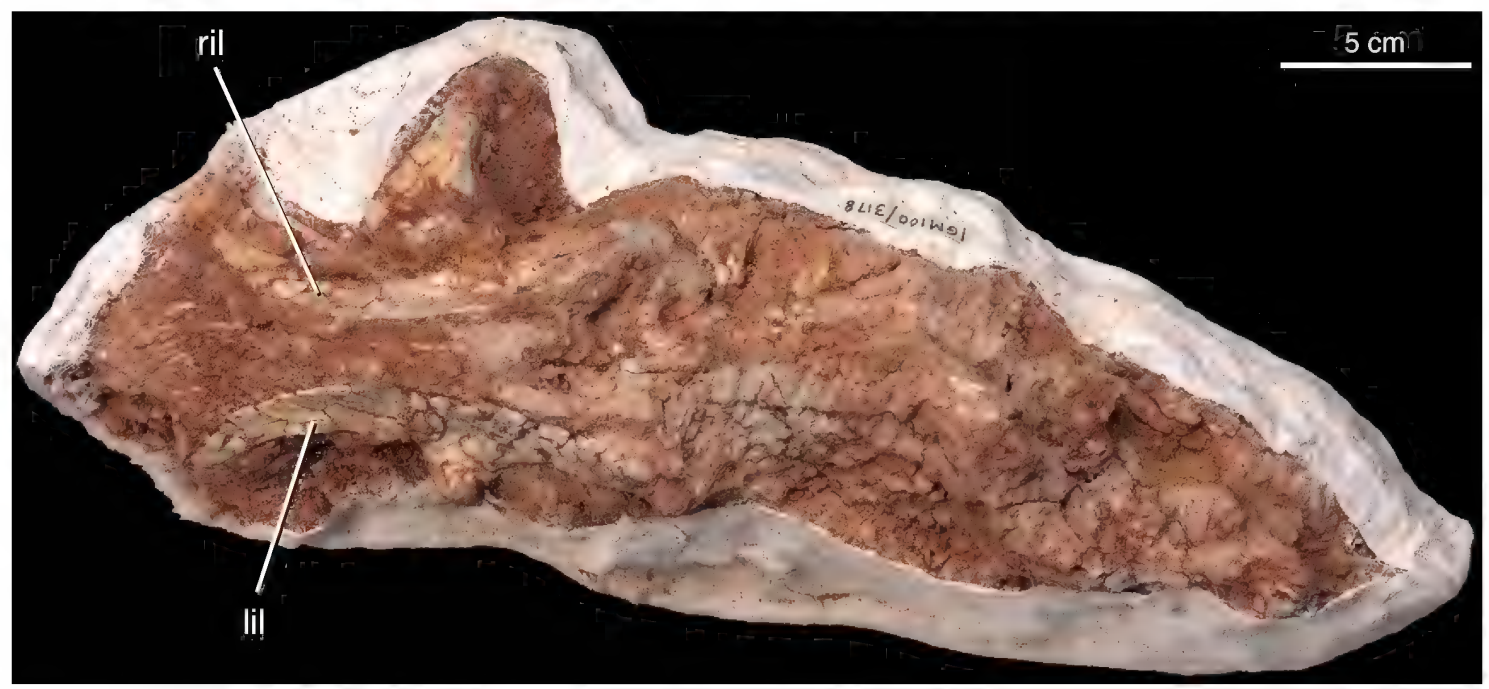

FIG. 39. Pelvis of IGM 100/3178 in dorsal view. Abbreviations in appendix 1.

of the seventh preserved vertebra of this specimen as the posteriormost dorsal, and instead identify it as the anteriormost sacral. We consider any vertebra with ribs that articulate with the ilium to be a sacral whether or not those ribs are actually fused to the ilium and whether or not their centra are fused to one another (e.g., Romer, 1956; Sereno, 2012). In contrast to all of these, the holotype of Abrictosaurus preserves only four unfused sacral vertebrae (Thulborn, 1974). However, this may be a juvenile feature of the specimen (Norman et al., 2004). Some specimens of Hypsilophodon retain the apparently plesiomorphic number of five sacrals, while others incorporate a posterior dorsal into the sacrum (Galton, 1974a). Galton (2014) described Haya and other early-diverging neornithischians as having developed a sacrum with six vertebrae through the addition of a caudosacral to the plesiomorphic five-vertebrae sacrum exemplified by Agilisaurus and Hexinlusaurus. However, given that IGM 100/2015 has two identifiable dorsosacrals and those earlier taxa have only one, it is likely that the six-vertebrae sacrum of Haya was developed instead through incorporation of an additional dorsosacral, as in heterodontosaurids (Santa Luca, 1980; Butler et al., 2012; Galton, 2014; but see Sereno, 2012, for an alternative interpretation of the first dorsosacral in Heterodontosaurus as the last nonsacral dorsal).

Therefore, Haya is more similar to heterodontosaurids than to other noncerapodan neornithischians in possessing six sacrals that include two recognizable dorsosacrals with small ribs originating on their transverse processes and articulating with the preacetabular blade of the ilium. This interpretation is based on the single Haya specimen, IGM 100/2015 (fig. 38), in which this region is most visible. Given the intraspecific variation in sacral number among both Thescelosaurus and Hypsilophdon, caution is warranted in assuming that this sacral count and configuration typifies all Haya. Likewise, little is known about the ontogeny of the sacrum in earlydiverging neornithischians, and the condition in Haya could reflect its skeletally immature status. Nevertheless, the morphology of the first two sacral ribs may be an important character for differential diagnosis of Haya from other noncerapodan neornithischians. We note that the vertebra and corresponding rib considered by Butler et al. (2011) to be the 15th dorsal of Changchunsaurus are morphologically similar to the first dorsosacral and rib of Haya. Combined with the evidence of two dorsosacrals in the seven-vertebrae sacra of a specimen of Thescelo- 


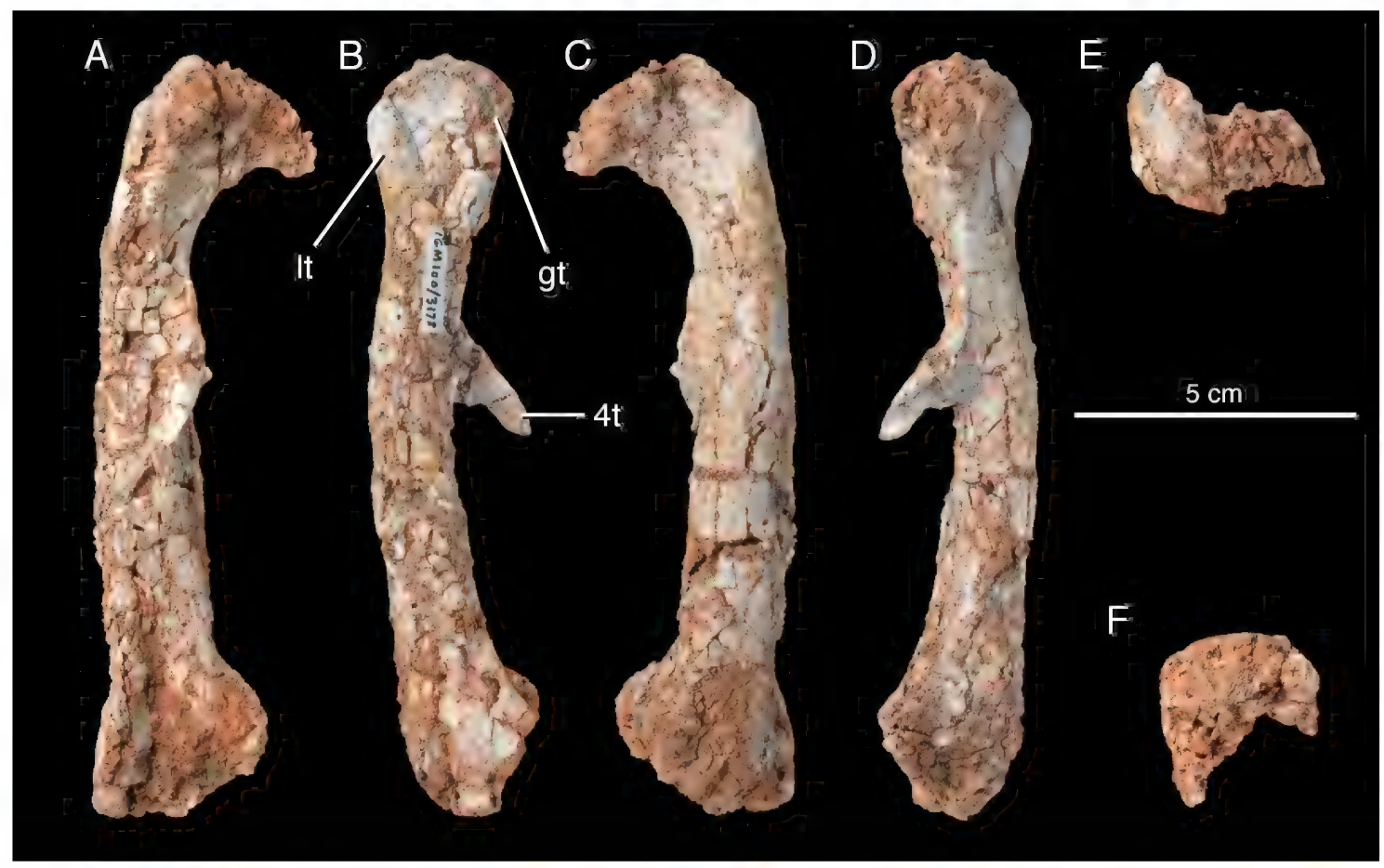

FIG. 40. Left femur of IGM 100/3178 in A, posterior, B, lateral, C, anterior, D, medial, E, proximal, and F, distal views. Abbreviations in appendix 1.

saurus (AMNH FARB 117) and Oryctodromeus (Varricchio et al., 2007), this suggests that the addition of dorsals to the sacrum among thescelosaurids may be a more common phenomenon than previously suspected.

Where visible, all sacral centra are fused or at least very tightly appressed to one another. Because not all of the sacral vertebrae are exposed in any Haya specimen, it is difficult to determine whether the sequence of fusion proceeded anteroposteriorly, as may have been the case in Parksosaurus (Brown et al., 2011), or in some other manner. The first dorsosacral centrum lacks the sharp ventral keel and laterally expanded articular facets of the successive sacral centra. The second and third sacral centra of Haya have narrow ridges on their ventral surfaces. The fifth and sixth sacral centra are transversely narrower than the first three. The lateral surfaces of the centra are smooth and lack nutrient foramina. All visible neurocentral sutures are closed in IGM 100/2015, whereas they may be open in the anterior (possibly the second and third) sacrals of IGM 100/3182 (fig. 50). There are deep circular fossae just anterior and posterior to the transverse processes on the neural arches of the first three sacrals. The low, anteroposteriorly expanded sacral neural spines are all widely separated in Haya, in contrast to the partially fused neural spines of Manidens, Oryctodromeus, and the holotype specimen of Thescelosaurus, USNM 7757 (Galton, 1974b; Varricchio et al., 2007; Pol et al., 2011). The third sacral rib is fused to both the second and third sacral centra, and also contacts the pubic peduncle of the ilium medially. A medial boss on the pubis contacts the second and third sacral centra, as in Orodromeus, Oryctodromeus, and Zephyrosaurus (Varricchio et al., 2007). Makovicky et al. (2011) also noted this articulation; however, they incorrectly described it as occurring at the junction of the first and second sacrals. Because 


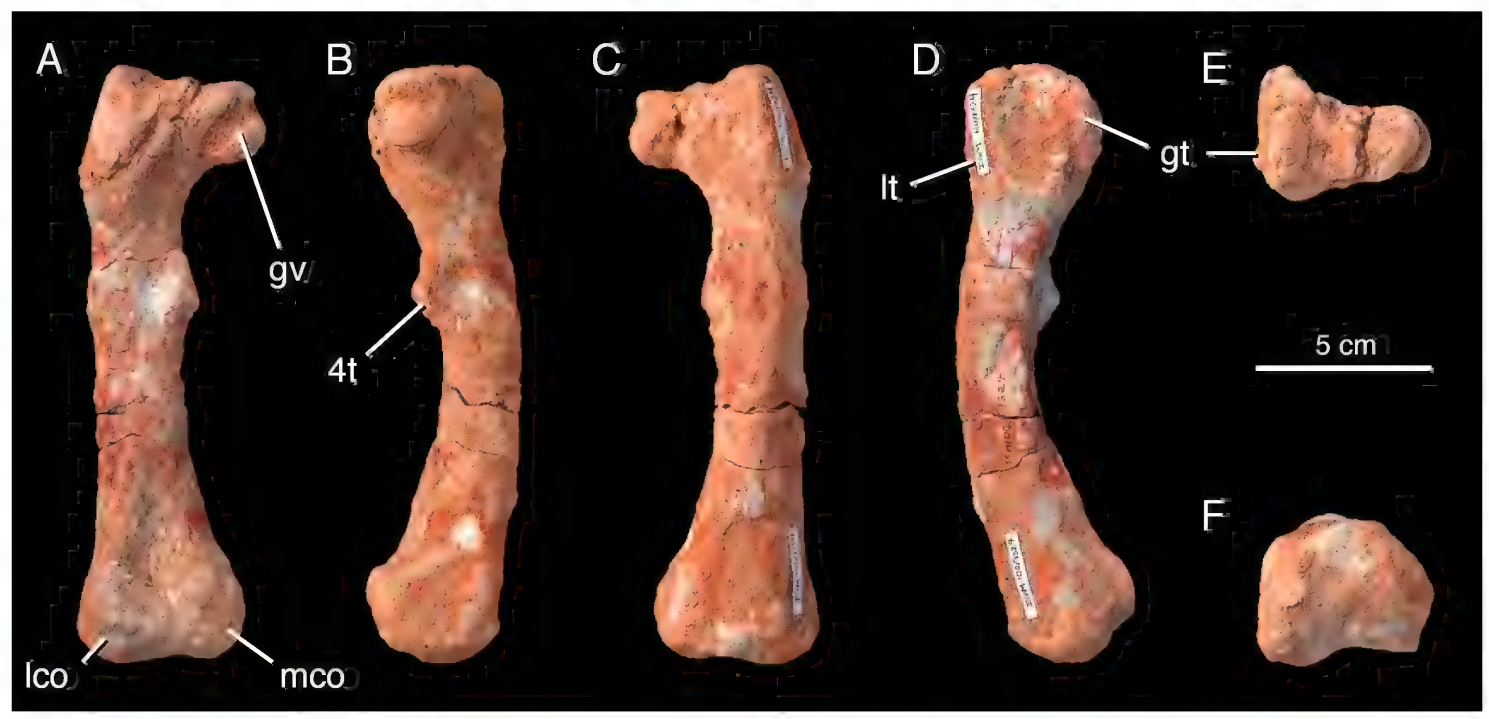

FIG. 41. Left femur of IGM 100/1324 in A, posterior, B, medial, C, anterior, D, lateral, E, proximal, and F, distal views. Abbreviations in appendix 1.

we consider the first dorsosacral to be part of the sacral series, as Makovicky et al. (2011) did, the vertebrae that articulate with the pubis are more properly described as the second and third sacrals. A similar articulation is present in Thescelosaurus (AMNH FARB 117), where the pubic peduncle of the ilium, the pubis, and the enlarged intersegmental third sacral rib all meet at a triple junction (Galton, 1974b). Whether all the elements meet at a triple junction in Haya cannot be assessed due to overlying matrix. A rib on the sixth sacral vertebra articulates with the medial surface of the ilium just ventral to the brevis shelf as in Orodromeus and Thescelosaurus (Galton, 1974b; Scheetz, 1999).

Caudal Vertebrae, Caudal Ribs, and Chevrons: A complete caudal series is not available from any Haya specimen yet collected. IGM 100/3137 preserves the most extensive portion of the tail, with 22 caudals (fig. 29). The full count was likely more than double this, based on near complete tails of Agilisaurus, Parksosaurus, Thescelosaurus, and Hypsilophodon that contain between 42 to 50 vertebrae (Gilmore, 1915; Norman et al., 2004). The first caudal vertebra is slightly dorsoventrally taller than the last sacral vertebra. It is not fused to the last sacral. Caudal centra become anteroposteriorly longer than dorsoventrally tall at approximately the sixth caudal, and this trend continues, with the midcaudal centra about twice as long as tall. Centra are smooth and gently concave laterally. The ventrolateral surface of the first caudal of IGM 100/2019 may have minute nutrient foramina, but these are not found in subsequent vertebrae, as they are in Jeholosaurus (Han et al., 2012). The anterior and posterior facets of the centra have slightly thickened borders. All centra have concave ventral borders in lateral view. The ventral surfaces of the centra are smooth, lacking the ventral grooves of Orodromeus and some Jeholosaurus (Scheetz, 1999; Han et al., 2012). Ventral grooves are variably present or absent in Jeholosaurus, and were considered to be an ontogenetic feature, as a smaller specimen lacks grooves that are present on larger specimens (Han et al., 2012). Where visible, the caudal centra of IGM 100/3137 are amphicoelous. All caudal neurocentral sutures are fused in IGM 100/2015. This is difficult to assess for IGM $100 / 2019$ and IGM 100/3137, because this region is largely obscured by matrix or eroded 


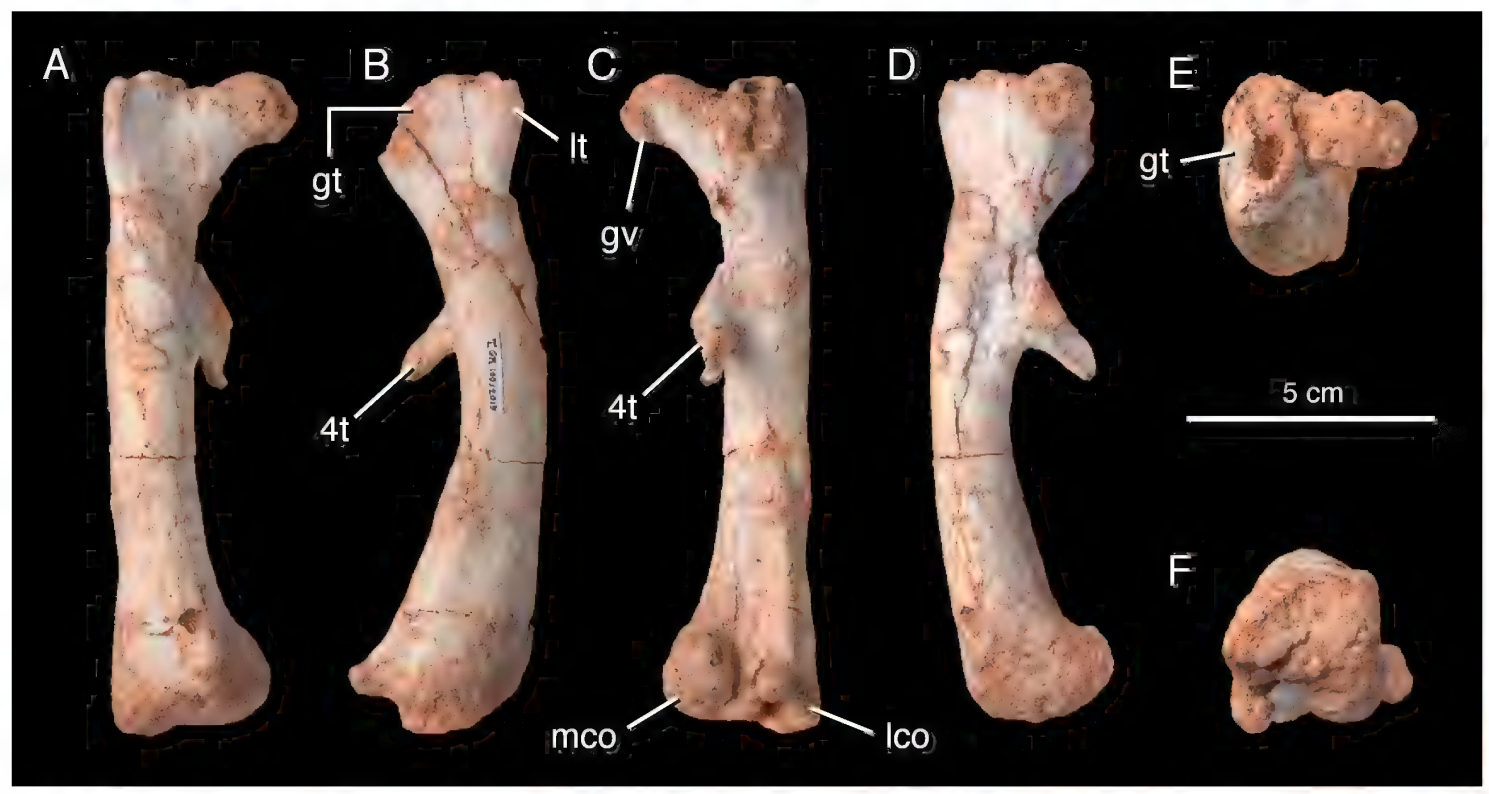

FIG. 42. Right femur of IGM 100/2015 in A, anterior, B, lateral, C, posterior, D, medial, E, proximal, and F, distal views. Abbreviations in appendix 1.

in these specimens. Nevertheless, some caudals in both do appear to have fused neurocentral sutures. The proximal caudal vertebrae have tall, laterally compressed, posterodorsally inclined neural spines (figs. 34, 38). Neural spines are present beyond at least the 15 th caudal vertebra. The 20th caudal of IGM 100/3137 appears to have neural spines that are less anteroposteriorly expanded than those of a similar serial position in Thescelosaurus (Gilmore, 1915), and are more similar to the shape inferred for caudals of approximately this position in Hypsilophodon (Galton, 1974a). In lateral view, the prezygapophyses are less steeply inclined relative to the horizontal than those of the dorsal vertebrae are. The articular facets of the pre- and postzygapophyses meet at a steep angle, becoming nearly vertical after the third caudal. This angle is steeper than on the corresponding anterior nine caudals of Albertadromeus, in which the anterior zygapophyses meet along a $45^{\circ}$ angle (Brown et al., 2013). The prezygapophyses of the distalmost preserved caudals of IGM 100/3137 are elongated relative to those of the anterior caudals (fig. 29).
The short, anterodorsally inclined transverse processes articulate with caudal ribs on the first 19 caudals of IGM 100/2015 (Makovicky et al., 2011). The first two of these ribs are relatively straight dorsoventrally. Subsequent ribs are gently arced dorsoventrally, until at least the eighth caudal and possibly beyond. Caudal rib length increases until the third rib, and then decreases beyond this (Makovicky et al., 2011). The first caudal rib is swept posteriorly as in Hypsilophodon and some specimens of Orodromeus (Galton, 1974a; Scheetz, 1999), but with a distal portion that curves anteriorly, so that the rib appears somewhat sigmoidal in dorsal view. Caudal ribs two through five curve somewhat anteriorly when viewed dorsally, whereas subsequent ribs are more transversely oriented. Neural canals of the anterior caudals are dorsoventrally elongate and teardrop shaped.

The first chevron is borne between the first and second caudal centra, as in most noncerapodan neornithischians, other than a specimen of Jeholosaurus that lacks chevrons on its first two caudals (Han et al., 2012). All chevrons articulate along facets housed on a pre- 


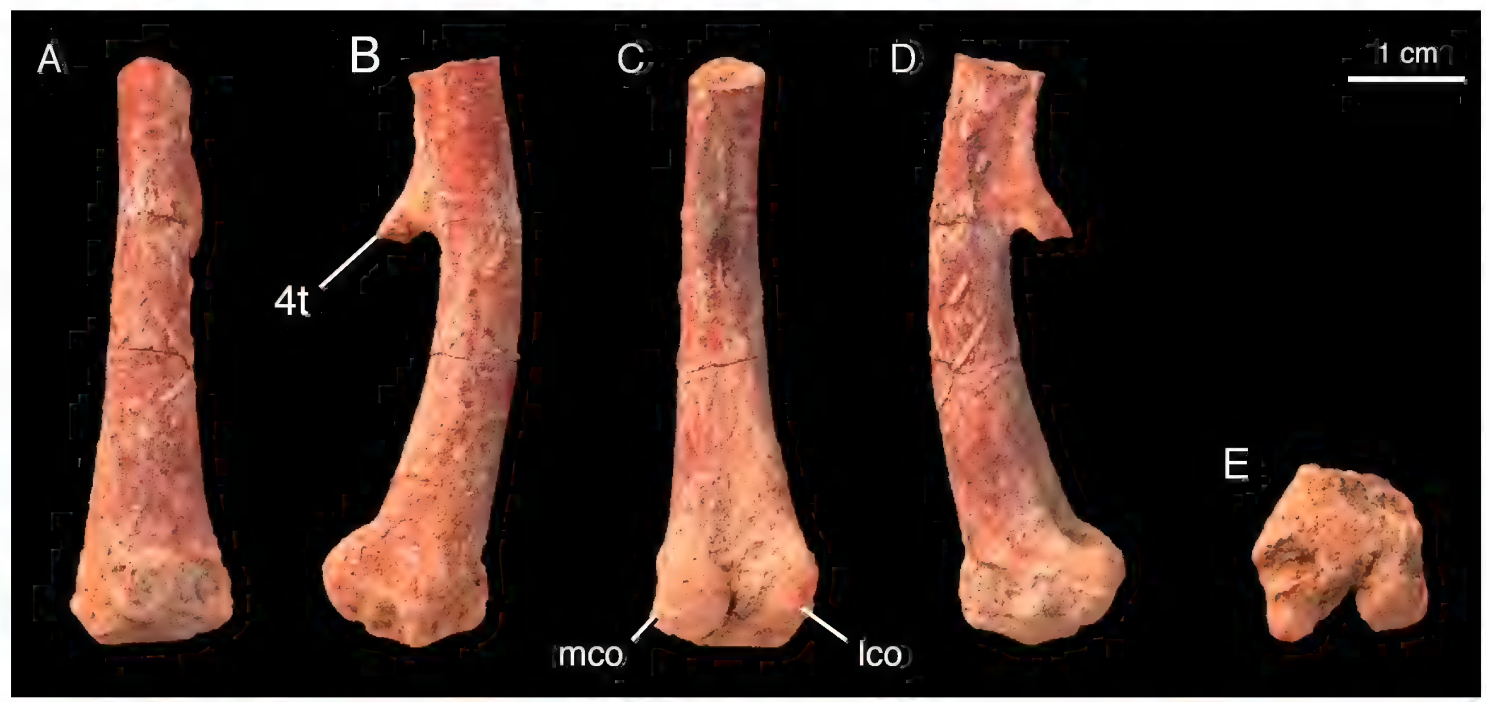

FIG. 43. Right femur of IGM 100/2020 in A, anterior, B, lateral, C, posterior, D, medial, and E, distal views. Abbreviations in appendix 1.

ceding and a succeeding vertebra (figs. 34,38 ). These facets meet at a nearly $90^{\circ}$ angle in lateral view, forming an inverted $\mathrm{V}$ shape for reception of the proximal end of the chevron. The third chevron is the longest, and the rest decrease in length very gradually. The first three chevrons are more posteriorly inclined than the rest, and the first two of these have the most pointed distal ends of the preserved chevrons. The distal ends gradually increase in anteroposterior width, becoming more paddle shaped around the 12th chevron. The haemal canals are dorsoventrally elongate, occupying nearly a third of the proximodistal length of the anterior chevrons, and almost half of the length of the posterior chevrons (after approximately the 10th chevron).

Ossified Tendons: Ossified tendons are present from the seventh dorsal to sixth sacral vertebrae in IGM 100/2015 and IGM 100/2019 (figs. 34, 35), and across all preserved dorsals of IGM 100/3181 (Norell and Barta, 2016). Ossified tendons are absent from the tails of all Haya specimens, as in Heterodontosaurus, Agilisaurus, Jeholosaurus, Albertadromeus, and Orodromeus (Peng, 1992; Scheetz, 1999; Han et al., 2012; Brown et al., 2013; Galton, 2014).
This contrasts with Tianyulong, Hexinlusaurus, Oryctodromeus, Parksosaurus, Thescelosaurus, and Hypsilophodon, in which ossified tendons are present on at least some caudal vertebrae (Gilmore, 1915; Parks, 1926; Galton, 1974a; He and Cai, 1984; Varricchio et al., 2007; Zheng et al., 2009). The tendons are round to mediolaterally flattened in cross section, and overlap to form a latticework across the neural spines. The medialmost tendons of this lattice slope posterodorsally; lateral to these, the middle set slopes posteroventrally, and the lateralmost set slopes posterodorsally, as in iguanodontoids (Organ, 2006). These ossified tendons, from medial to lateral, are likely the tendons of M. spinalis and two layers of M. semispinalis (one superficial), inferred on the basis of comparison with extant avian tendons (Organ, 2006; Holmes and Organ, 2007). Some tendons of the lateralmost set extend across up to six vertebrae. Those of the middle set extend across two or three vertebrae. Some tendons of the lateralmost set bifurcate posteriorly. In contrast to Albertadromeus (Brown et al., 2013), there may be no strictly parasagittally oriented tendons in Haya. 


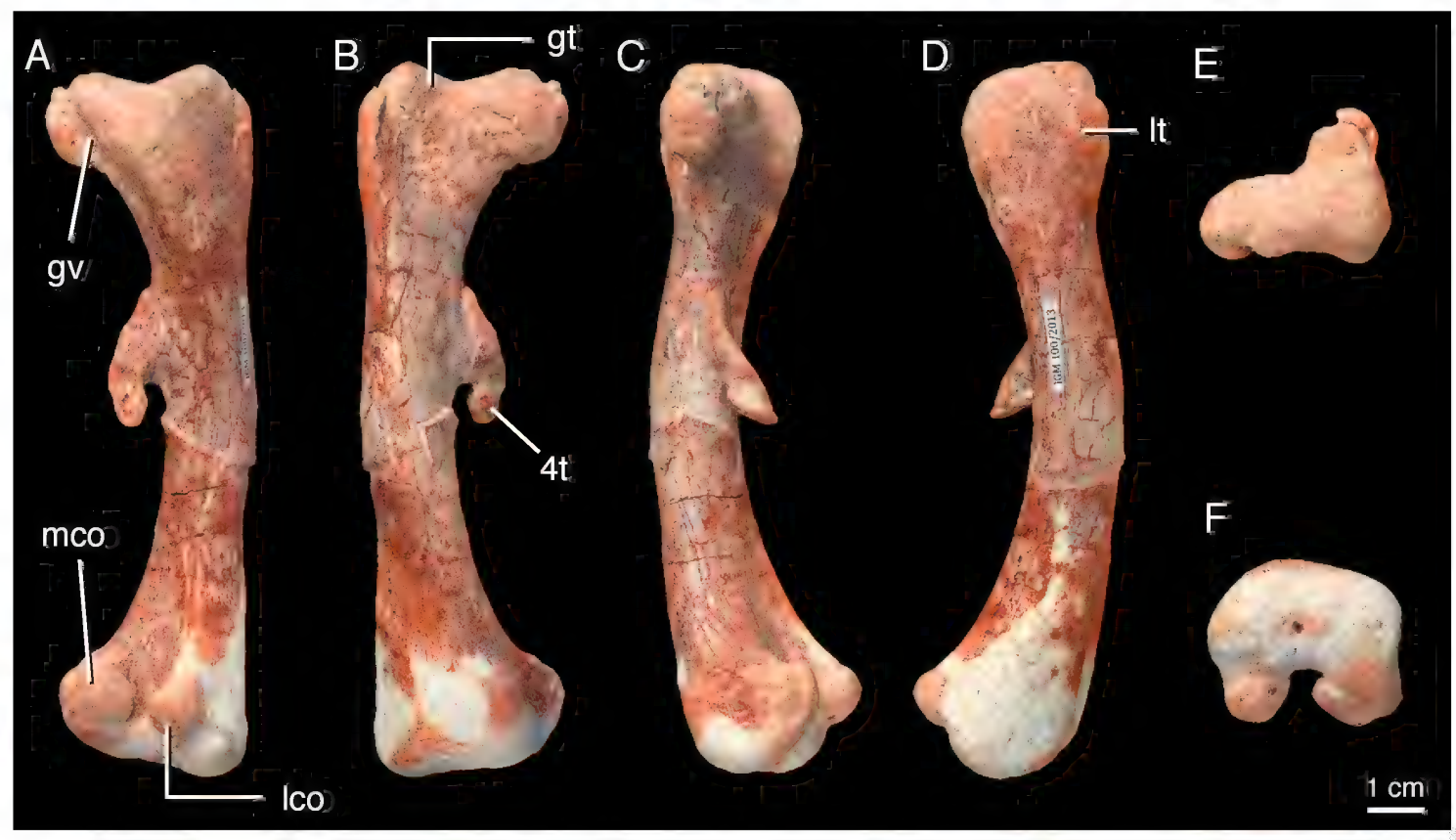

FIG. 44. Right femur of IGM 100/2013 in A, posterior, B, anterior, C, medial, D, lateral, E, proximal, and F, distal views. Abbreviations in appendix 1.

\section{Appendicular Skeleton}

SCAPUla: As in other noncerapodan neornithischians, the scapula is approximately equal in length to the humerus, and is medially concave throughout its length (when viewed anteriorly) to wrap around the ribcage (Norman et al., 2004; Makovicky et al., 2011; figs. 35, 36). It lacks the slight lateral bend toward the distal end seen in Hexinlusaurus and Hypsilophodon (Galton, 1974a; He and Cai, 1984). The scapula thins mediolaterally near its distal end. Orienting the scapula vertically, the anterior margin is straight, while the posterior border is concave posteriorly. The proximal end of the scapula flares anteroposteriorly to contact the coraocoid. The anterior and posterior margins of the scapula have fine striations and rough patches, possibly indicating attachment sites for the trapezius and levator scapulae anteriorly, and the scapulohumeralis caudalis and teres major posteriorly (Fearon and Varricchio, 2016), though these features are not as well defined as in Oryctodromeus. The distal end of the scapula is asymmetrical in lateral view, with a wider posteriorly directed flange extending from the posterior surface. This flange is not as pronounced as that of Orodromeus, Oryctodromeus, and Thescelosaurus (Gilmore, 1915; Scheetz, 1999; Varricchio et al., 2007), and the scapular shaft of Haya is proportionally longer and anteroposteriorly narrower overall than in these taxa. However, the scapula of Haya is greatly expanded compared to the narrow, straplike scapulae of Eocursor and Heterodontosaurus (Santa Luca, 1980; Butler, 2010). The distal end of the scapula is also less squared off than those of Changchunsaurus and Koreanosaurus (Butler et al., 2011; Huh et al., 2011). The scapula of Haya is very similar in overall shape to that of Hexinlusaurus (He and Cai, 1984), except that Haya has a slightly more anteriorly expanded acromion. The lateral surface of the scapula is smooth, lacking the prominent scapular spine that extends from the acromion process in Oryctodromeus (Varricchio et al., 2007). Though the overall shape of the scapula is very similar to that 


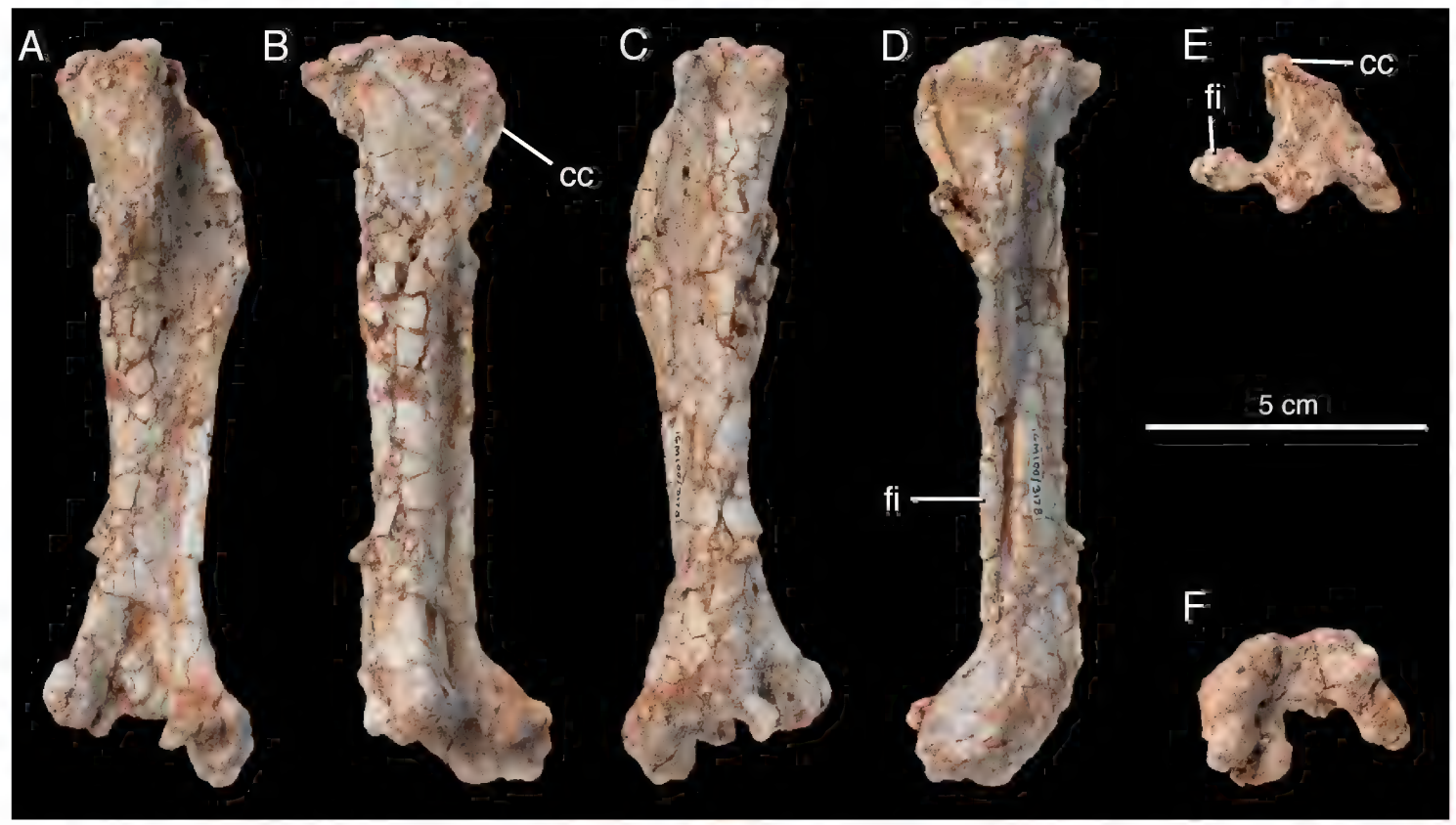

FIG. 45. Left tibia and fibula of IGM 100/3178 in A, anterior, B, medial, C, posterior, D, lateral, E, proximal, and $\mathbf{F}$, distal views. Abbreviations in appendix 1.

of Parksosaurus (ROM 804), there is no trace of the ossified suprascapular element of that taxon (Parks, 1926). The distal border of the scapula is roughened and irregular, but whether this is because the scapula bore a cartilaginous extension, as hypothesized for other taxa (Norman et al., 2004), or because of insect or other taphonomic damage remains uncertain. Proximally, the deltoid fossa is a depression extending across the lateral surface of the proximal plate (sensu Han et al., 2012), onto the acromion process and supraglenoidal buttress (sensu Butler, 2010). Between these two processes is a rounded, laterally projecting tubercle articulating with a matching boss on the coracoid. Haya lacks the smaller supraglenoidal buttress and distinct supraglenoid fossa of Yueosaurus (Zheng et al., 2012). The glenoid fossa faces posteriorly and slightly laterally.

CorACOID: The coracoid is a platelike bone that thins outward from the glenoid along its anterior and posterior margins (fig. 35). The two coracoids are unfused and meet along the ventral midline. The scapula may be partially fused to the coracoid in IGM 100/2015, as in Koreanosaurus and Oryctodromeus (Varricchio et al., 2007; Huh et al., 2011; Makovicky et al., 2011), though a suture between the bones remains visible. This differs from most other early-diverging neornithischians, where these elements are unfused (Han et al., 2012). However, whether or not fusion of the scapula and coracoid may have occurred later ontogenetically in these taxa remains unknown (Huh et al., 2011). A small, round coracoid foramen is present just ventral to the scapulocoracoid suture. The coracoid contributes to half the articular facet of the glenoid. The posterior border of the coracoid ventral to the glenoid fossa is strongly concave, as in many other early-diverging neornithischians (Gilmore, 1915; Galton and Jensen, 1973; Galton, 1974a; Scheetz, 1999; Varricchio et al., 2007; Huh et al., 2011). This embayment is absent or poorly developed in Lesothosaurus, Heterodontosaurus, and Hexinlusaurus, but is present in Manidens and "Yandusaurus" hongheensis (Santa Luca, 1980; He and Cai, 1984; Pol et al., 2011; Baron et al., 2017). Haya lacks the coracoid 


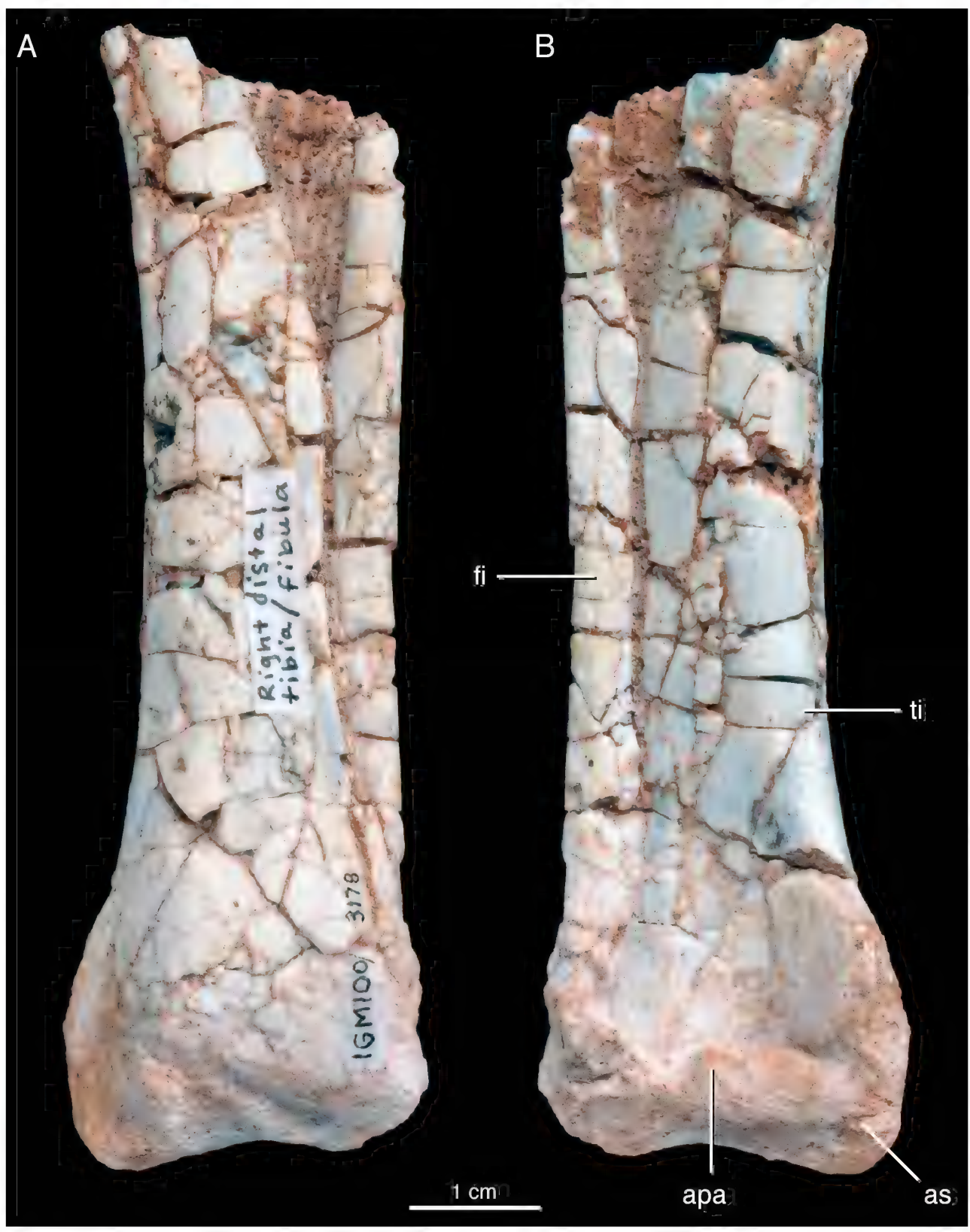

FIG 46. Right distal tibia, fibula and astragalus of IGM 100/3178 in A, posterior and B, anterior views. Abbreviations in appendix 1.

tubercle on the posteroventral corner of the coracoid seen in other taxa (Santa Luca, 1980; Norman et al., 2004; Makovicky et al., 2011). This tubercle is possibly the origin of the coracobrachialis muscle in these taxa (Santa Luca, 1980), though the hypothesized region of origin for this muscle is smooth in Haya and Oryctodromeus (Fearon and Varricchio, 2016). As noted by Makovicky et al. (2011), much of the ventral border of the coracoid is damaged in IGM 100/2015, resulting in what are possibly traces of insect feeding. 


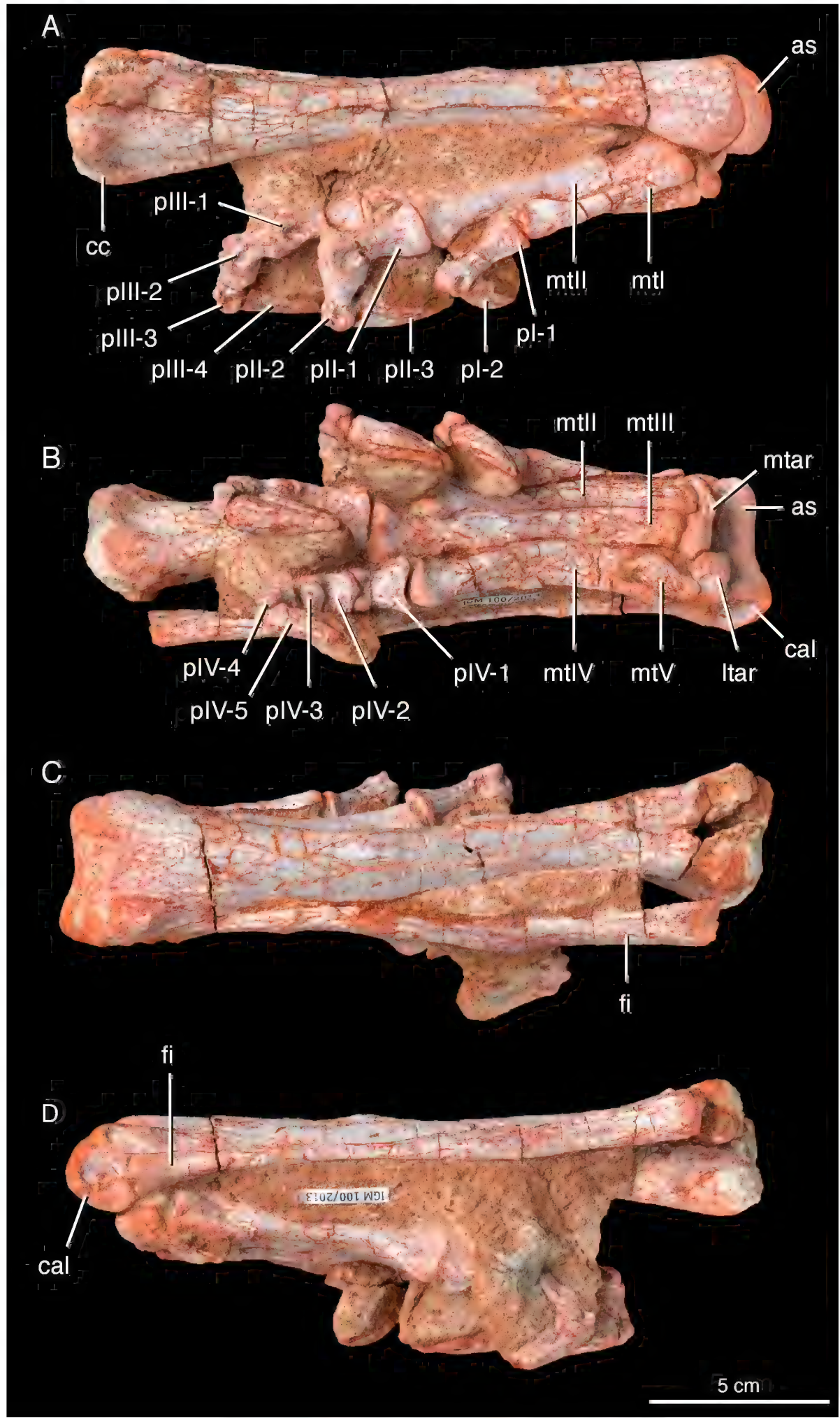


STERnUM: IGM 100/2015 has two flat, reniform sternal plates articulated along their anterior margins (fig. 35). The sternum is slightly thicker anteriorly near its articulation with the coracoid. The sternum has a strongly concave lateral border and strongly convex medial border. These borders are more strongly curved than the sternal margins of Othnielosaurus and Orodromeus (Galton and Jensen, 1973; Scheetz, 1999), and are fairly similar in overall shape to those of Changchunsaurus, Parksosaurus, and Dryosaurus (Gilmore, 1925; Parks, 1926; Galton, 1981; Butler et al., 2011). The posterior border of the sternum of Hypsilophodon is not as rounded as that of Haya (Galton, 1974a; Makovicky et al., 2011). Haya lacks the bumps on the ventral and medial surfaces of the sternum present in Hypsilophodon (Galton, 1974a). There also is no fenestra in the sternum, in contrast to a possible fenestra present in Heterodontosaurus (Sereno, 2012). As described above, no sternal ribs have been found in any Haya specimen, nor are there any clear indications on the sternum of their points of articulation (Makovicky et al., 2011).

Humerus: IGM 100/2015 (fig. 35) preserves the most information about the humerus and forms the basis for this description. In this specimen, the humerus is articulated with the glenoid fossa, with its distal end pointing posteriorly. The overall humeral shape of Haya differs little from those of other early-diverging neornithischians, which further differ little from one another, with only few exceptions. This observation is supported by both traditional and geometric morphometric analyses of humeral shape that included Haya (Fearon and Varricchio, 2015). The humeral head is rotated $90^{\circ}$ relative to the distal end of the humerus, as in other earlydiverging ornithischians and ornithopods (Norman et al., 2004), but in contrast to Lesothosaurus (Baron et al., 2017). The head is mediolaterally broad and proximally rounded. The lateral tuberosity is poorly defined. This differs from Kore- anosaurus, in which the lateral tuberosity is prominent and well separated from the humeral head (Huh et al., 2011). The extent of the medial tuberosity in Haya cannot be fully determined because it lies in contact with the matrix, but it was probably small and poorly separated from the humeral head, again in contrast to the welldefined medial tuberosity of Koreanosaurus (Huh et al., 2011). The anterior face ventral to the head and medial to the deltopectoral crest is flat, whereas it forms a shallowly concave bicipital sulcus in other taxa, including Jeholosaurus, Koreanosaurus, Changchunsaurus, and Hypsilophodon (Galton, 1974a; Butler et al., 2011; Huh et al., 2011; Han et al., 2012). A proximodistally broad deltopectoral crest extends along the proximal half of the humerus. It is not as expanded as that of Oryctodromeus, Koreanosaurus, and Orodromeus (Scheetz, 1999; Huh et al., 2011; Fearon and Varricchio, 2015), but is more expanded than those of Othnielosaurus, Thescelosaurus, and Dryosaurus (Galton and Jensen, 1973; Galton, 1974b, 1981). The deltopectoral crest reaches its apex about one third of the way distally along the total length of the humerus. The border of the crest is slightly concave in lateral view, in contrast to the convex border of Heterodontosaurus (Galton, 2014). The deltopectoral crest bends anterolaterally from the humeral shaft, and merges gradually with it distally, unlike the sharp transition between crest and shaft seen in Heterodontosaurus (Santa Luca, 1980). The lateral surface of the deltopectoral crest contains a shallow trough housing muscle scars that may mark the insertion sites of the deltoideus clavicularis and deltoideus scapularis muscles (Fearon and Varricchio, 2016). Distal to the deltopectoral crest, the shaft is elliptical in cross section and relatively gracile like those of Jeholosaurus, Changchunsaurus, Orodromeus, and Hypsilophodon (Galton, 1974a; Scheetz, 1999; Butler et al., 2011; Han et al., 2012). In contrast, the humeral shafts of Oryctodromeus and Koreanosaurus are

FIG. 47. Right tibia, fibula, tarsus, and pes of IGM 100/2013 in A, medial, B, anterior/flexor, C, posterior, and $\mathrm{D}$, lateral views. Abbreviations in appendix 1. 


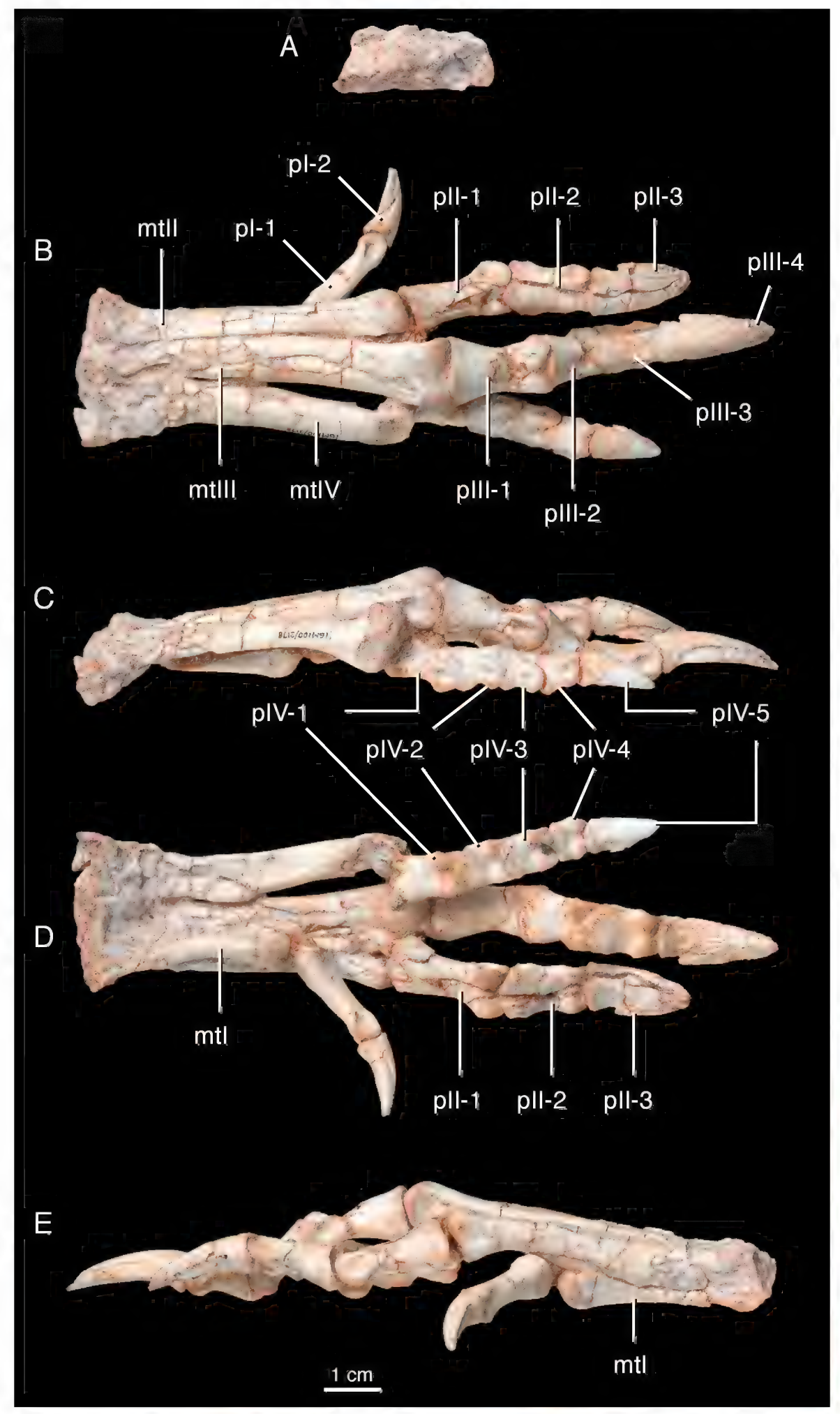


more robust relative to their length (Huh et al., 2011; Fearon and Varricchio, 2015). The ulnar and radial condyles are separated by a shallow anterior intercondylar groove. The ulnar and radial condyles appear subequal in size, though Makovicky et al. (2011) considered the ulnar condyle to be slightly larger, as in many other taxa (e.g., Galton, 1974a; Scheetz, 1999). This differs from the condition in Koreanosaurus, in which the ulnar condyle is much broader transversely (Huh et al., 2011); and from Lesothosaurus, in which the radial condyle is noticeably larger than the ulnar condyle (Baron et al., 2017). The ulnar condyle projects farther anteriorly (Makovicky et al., 2011). As noted by Makovicky et al. (2011), matrix obscures the posterior side of the condyles, preventing confirmation of the presence or absence of an olecranon fossa. Makovicky et al. (2011) also describe two holes in the anterior face of the proximal end as possible damage from scavenging insects. The surfaces of the head and condyles are rough, indicating probable cartilage attachment sites.

ULNA: A well-preserved ulna from a large individual (IGM 100/3661, fig. 14) is very similar in shape in lateral view to the ulna of Orodromeus (Scheetz, 1999). As in other early-diverging neornithischians except for Heterodontosaurus, the olecranon process extends a short distance beyond the saddle-shaped joint surface for the humerus (Norman et al., 2004). There is a low coronoid process on the medial surface of the proximal end. The posterior border of the ulna of IGM 100/3661 is "keeled" as in Orodromeus and Koreanosaurus (Scheetz, 1999; Huh et al., 2011), but in contrast to other Haya specimens, in which the proximal end is more gently curved and less expanded in lateral view. The lateral surface of the ulna of IGM 100/3661 is grooved longitudinally, but those of other specimens are not. In IGM 100/3181 (Norell and Barta, 2016), a ridge that wraps around the ulna from the lateral surface proximally to the dorsal surface distally gives the appearance that the ulna is twisted. The cross section changes from triangular near the proximal articular surface to elliptical along much of its length. The distal end of the ulna of IGM $100 / 3661$ is dorsoventrally expanded. No other specimen preserves a complete ulnar distal end. The shaft of the ulna is mostly straight between the expanded ends, giving it a less bowed appearance overall than that of Hypsilophodon (Galton, 1974a). The ulna of Haya is proportionally more elongate, with relatively narrower expansions of its proximal and distal ends, than that of a large specimen of Thescelosaurus (AMNH FARB 5034). Haya lacks the broad depression that covers the lateral surface of the distal third of the ulna of Thescelosaurus (Gilmore, 1915).

RADIUS: The radius is mediolaterally compressed along its length, but the distal half is mediolaterally thicker than the proximal half. The distal half twists slightly laterally. A longitudinal ridge runs the length of the shaft dorsally. The proximal end is more dorsoventrally expanded than the elliptical distal end of IGM 100/2015 (fig. 35). The proximal end contains a shallow sulcus where it articulates with the humerus, as in other early-diverging neornithischians (Norman et al., 2004; Makovicky et al., 2011). The proximal end is very shallowly concave where it articulates with the ulna. The proximal expansion is not as dramatic as that of a specimen of Thescelosaurus (AMNH FARB 5034). The distal end of the radius is rugose in IGM 100/2015, and its posterior corner forms a slightly raised tubercle. Because of their differing positions, this feature may not be homologous to the more proximally located radial tubercle of Heterodontosaurus (Santa Luca, 1980). The radius of Haya is proportionately more elongate than that of one large Thescelosaurus (AMNH FARB 5034), and is more similar to the slender radius of the smaller holotype of Thescelosaurus, USNM 7757 (Gilmore, 1915), possibly indicating

FIG. 48. Right pes of IGM 100/3178 in A, proximal, B, dorsal, C, lateral, D, ventral, and E, medial views. Abbreviations in appendix 1. 


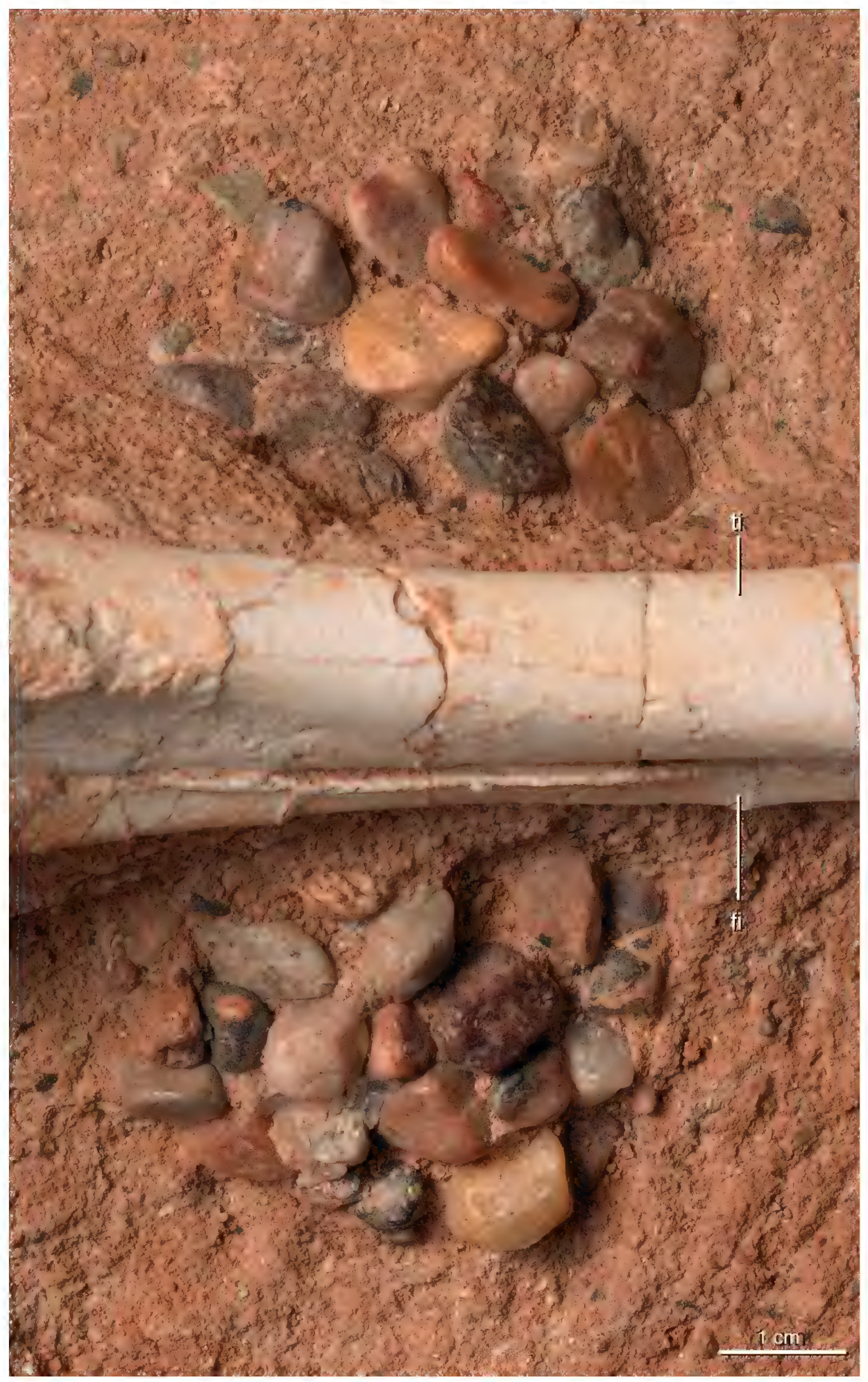

FIG. 49. Gastroliths underlying the left tibia and fibula of IGM 100/2015. Abbreviations in appendix 1. 


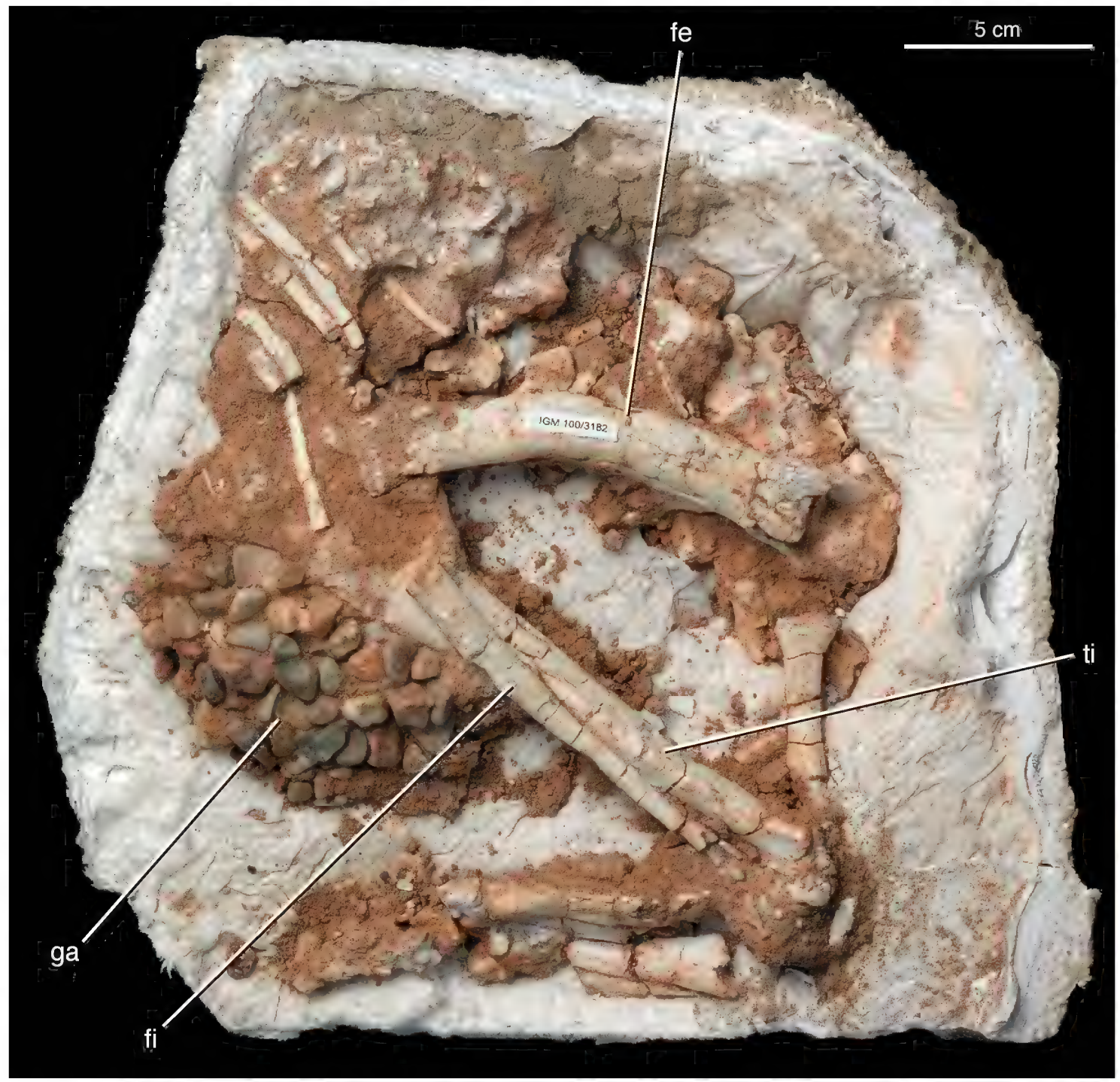

FIG. 50. Ribs, sacral vertebrae, left hind limb, and associated gastroliths of IGM 100/3182. Abbreviations in appendix 1 .

that the radius becomes more robust with increasing size (Galton, 1974b). The radius of IGM $100 / 2015$ is similar in overall shape to that of Hypsilophodon (Galton, 1974a). The radius of Haya lacks defined carpal articular facets.

CARPAls: Only IGM 100/3181 preserves any carpals, and these were dislodged from their life positions. One of these is pyramidal with two distinct concave articular facets (Norell and Barta, 2016: fig. 6A, top). We tentatively identify this element as the intermedium, based on its morphological similarity to the intermedia of Thescelosaurus (AMNH FARB 5034) and Hypsilophodon (Gilmore, 1915; Galton, 1974a). The intermedia of both taxa, like the element in Haya, are diamond shaped in dorsal view. The two articular facets of the possible Haya intermedium probably received the radius and ulna. The other carpal element of IGM 100/3181 is cuboid, with at least two concave articular facets on a 
side opposite a concave surface with two distinct foramina (Norell and Barta, 2016: fig. 6A, bottom). The fact that it is about the same size as the intermedium suggests that it is either the radiale or ulnare. The ulnare of Heterodontosaurus possibly contains two foramina as in the carpal of Haya (Santa Luca, 1980), but the morphologies of the two elements are otherwise dissimilar, making it hard to determine whether or not the foramina are in homologous locations. The square shape of the Haya carpal agrees more closely with the shape of the ulnare of Tenontosaurus in proximal and distal views, rather than the more triangular radiale of Tenontosaurus (Forster, 1990). No view of the bone precisely matches the dorsal views of the Orodromeus (Scheetz, 1999) or Thescelosaurus (Gilmore, 1915) radiale or ulnare, however. Therefore, though we are confident that this bone is likely the radiale or ulnare, we caution that the lack of topological data for this bone and the current dearth of well-preserved early-diverging neornithsichan carpi hinders comparisons that could allow for a more definitive identification.

MetaCARPals: Metacarpals are presently known from only two specimens: IGM 100/2015 has metacarpals I through IV (fig. 37), and IGM 100/3181 preserves metacarpals I and II (Norell and Barta, 2016: fig. 6B, C). The following description orients the hand so that the palmar surface is considered ventral, and the antipalmar surface dorsal, with digit I medial. The proximal end of metacarpal I is roughly triangular, with the point directed medially. The lateral condyles of metacarpals I and II are longer than the medial condyle, forcing phalanx I-1 into a medially directed orientation as in many other dinosaurs (e.g., Barta et al., 2018). The condyles of these elements are more rounded in IGM $100 / 2015$ than in IGM 100/3181. The distal ends of metacarpals I and II are also twisted medially relative to the proximal end. The distal articular surfaces of metacarpals I and II are ginglymoid and offset from the shaft by a pronounced dorsal lip. The shaft of metacarpal II is more constricted relative to the proximal and distal ends than that of metacarpal I. The collateral ligament pits on the first and second metacarpals are deep and elliptical, with those of the lateral sides larger than those of the medial sides. As in Hexinlusaurus, Orodromeus, Thescelosaurus, and Hypsilophodon (Gilmore, 1915; Galton, 1974a; He and Cai, 1984; Scheetz, 1999), metacarpals II and III are the longest, being subequal in length (Makovicky et al., 2011). These taxa may differ from Lesothosaurus, in which metacarpal III was reconstructed as noticeably longer than II (Baron et al., 2017), though measurements and a reconstruction provided by Sereno (1991) suggest they are nearly equal in length. Metacarpal II has a deep depression for the insertion of extensor digitorum longus on the dorsal surface of its proximal end, much as in many other earlydiverging dinosaurs (Barta et al., 2018). This depression is shallower in metacarpal I. None of the metacarpals have deep distal extensor depressions like those of Heterodontosaurus (Santa Luca, 1980). Metacarpal III is mediolaterally narrower overall than metacarpal II. Metacarpal IV is the shortest of the preserved metacarpals in Haya (Makovicky et al., 2011), though metacarpal V, if present, would have likely been shorter, as evidenced by the other aforementioned taxa for which metacarpals are preserved. No evidence yet exists for a fifth metacarpal in Haya, however. Metacarpal IV is not as distally expanded as the other metacarpals. The shape of its distal articular surface in IGM $100 / 2015$ is partially obscured by matrix.

Manual Phalanges: The phalangeal formula for Haya, based on all available material is 2?-3?-4?-?-?. No complete phalangeal sequence has been preserved for any digit of either IGM 100/2015 (fig. 37) or IGM 100/3181 (Norell and Barta, 2016: fig. 6B-E). IGM 100/3137 (fig. 29) and IGM 100/3672 also have a few disarticulated phalanges. The proximalmost phalanges of digits I and II are about the same length, with that of digit III markedly shorter. An isolated phalanx of IGM 100/3181 (Norell and Barta 2016: fig. 6D), likely from digit III, is wider across its proximal end than it is long. All phalanges have gingly- 
moid articulations (Makovicky et al., 2011) and deep circular collateral ligament pits. The proximal articular facets of the phalanges are triangular, with a raised dorsal and flat ventral surface. The ungual phalanges are pointed and gently curved, similar to those of Hypsilophodon (Galton, 1974a). Their medial and lateral edges are sharp and slightly expanded beyond the width of the articular surface. IGM 100/3181 preserves three unguals, two subequal in size, and one smaller one (Norell and Barta, 2016: fig. 6E). These are probably from digits I-III, but we cannot be sure which ungual belongs to which digit at this time.

ILIUM: The dorsal border of the ilium is strongly convex in lateral view along the preacetabular process, but straightens at approximately the level of the acetabulum, continuing as the more gently convex dorsal border of the postacetabular process (figs. 34, 38, 39). This is similar to the shape of the dorsal iliac border of Lesothosaurus, Hexinlusaurus, Othnielosaurus, Jeholosaurus, and Orodromeus (Galton and Jensen, 1973; He and Cai, 1984; Scheetz, 1999; Han et al., 2012; Baron et al., 2017). In contrast, the dorsal border of the ilium is sigmoidal to posteriorly concave in Thescelosaurus (AMNH FARB 117) and Oryctodromeus, and convex across its whole length in the indeterminate orodromine TMP 2008.045.002 and some Hypsilophodon specimens (Brown et al., 2013; see their figure 8 for comparisons among many of these taxa). Most of these differences in outline are caused by the degree of either dorsal or ventral deflection of the postacetabular process. The ilium of Haya is laterally concave, due to lateral bending of the pre- and postacetabular processes (Makovicky et al., 2011). In lateral view, the anterior end of the preacetabular process is more hooked in IGM 100/2019 (fig. 34) than in IGM 100/2015 (fig. 38 ). It is also more hooked than that of most thescelosaurid taxa (Brown et al., 2013). The overall curvature of the preacetabular process is also individually variable in Orodromeus (Scheetz, 1999), suggesting that variation in this region in Haya is not unusual. In contrast to the description of Makovicky et al. (2011), we note that the preacetabular blade twists slightly, so that part of its lateral face and ventral edge are visible in dorsal view (see Galton, 1974a: fig. $50 \mathrm{~A}$, for an example of this). This matches the usual morphology of most early-diverging neornithischians (Norman et al., 2004). The posterior edge of the postacetabular process is squared off in lateral view on the right ilium of IGM $100 / 2019$ (fig. 34), the only ilium that is relatively undamaged in this region. This differs from the squared-off, but dorsoventrally expanded posterior margins of Oryctodromeus and Thescelosaurus, and the more tapered posterior border of TMP 2008.045.002 (Brown et al., 2013). The brevis fossa of Haya is mediolaterally broad and gently concave. It slopes medioventrally, with its medial edge just barely visible in lateral view (fig. 38). The brevis fossa is more visible in lateral view in Thescelosaurus (AMNH FARB 117; Galton, 1974b). The pubic peduncle is anteroposteriorly compressed and pointed in lateral view (fig. 38). It is directed strongly anteriorly as in Lesothosaurus, Jeholosaurus, Hypsilophodon and most thescelosaurids (Galton, 1974a; Han et al., 2012; Brown et al., 2013; Baron et al., 2017). In Haya, the pubic peduncle meets the preacetabular blade at an approximately $40^{\circ}$ angle, slightly greater than the $30^{\circ}$ angle of Jeholosaurus (Han et al., 2012). The ischiac peduncle is mediolaterally expanded compared with the pubic peduncle. As in other neornithischians, a massive, laterally expanded antitrochanter is developed on the ischiac peduncle, which differs from the narrow, ventrally concave antitrochanter of Heterdontosaurus (Santa Luca, 1980; Galton, 2014). The acetabulum is fully open, in contrast to the partially closed acetabula of Eocursor, Lesothosaurus, Agilisaurus, and Hexinlusaurus (He and Cai, 1984; Peng, 1992; Barrett et al., 2005; Butler, 2010; Baron et al., 2017). There is no distinct laterally expanded supraacetabular rim in Haya, in contrast to the weakly developed rims of Jeholosaurus, TMP 2008.045.002, Orodromeus, and Thescelosaurus (AMNH FARB 117) (Scheetz, 1999; Han et al., 2012; Brown et al., 2013), and 
the more prominent supraacetabular flanges of Eocursor, Lesothosaurus, Heterodontosaurus, Agilisaurus, and Scutellosaurus (Santa Luca, 1980; Peng, 1992; Barrett et al., 2005; Butler, 2010; Baron et al., 2017). The medial surface of the ilium is largely obscured by matrix in all studied specimens. The lateral surfaces of the ilia are smooth in IGM 100/2015 and IGM 100/2019, with no obvious muscle scarring.

PuBIs: The pubis consists of an anterolaterally directed prepubic process, a cuboidal main body, and a long posteriorly projected postpubic rod (figs. 34, 38). The prepubic process is relatively long, extending past the anterior border of the ilium and reaching the position of the 14th (in IGM 100/2019) or 15th (in IGM 100/2015) dorsal vertebra, anteriorly. It is somewhat mediolaterally compressed, with a shallow ventral groove as in other neornithischians (Norman et al., 2004; Makovicky et al., 2011). The end of the process is squared off, lacking the slight dorsoventral expansion seen in Thescelosaurus (AMNH FARB 117). Its lateral surface is covered in longitudinal striations, possibly marking the origins of M. ambiens (Romer, 1927; Maidment and Barrett, 2011). The main body of the pubis is cuboidal, faces anteromedially, and articulates with the pubic peduncle of the ilium (Makovicky et al., 2011). A robust process extends medially from the main body of the pubis to articulate with the junction between the second and third sacral vertebrae, as in Orodromeus, Zephyrosaurus, and Oryctodromeus (Scheetz, 1999; Varricchio et al., 2007; Makovicky et al., 2011). Just posterior to this is the obturator notch, which remains open, as in some Lesothosaurus, some Orodromeus, some Thescelosaurus, some Hypsilophodon, and possibly Jeholosaurus (Gilmore, 1915; Galton, 1974a, 1974b; Scheetz, 1999; Brown et al., 2011; Han et al., 2012; Baron et al., 2017); but in contrast to Eocursor, Heterodontosaurus, Hexinlusaurus, Oryctodromeus, some Lesothosaurus, some Orodromeus, some Thescelosaurus, and some Hypsilophodon, where it is a closed foramen (Galton, 1974a, 1974b; Santa Luca, 1980; He and Cai, 1984; Scheetz, 1999;
Varricchio et al., 2007; Butler, 2010; Baron et al., 2017). Clearly, the open or closed status of the obturator notch/foramen is highly variable in ornithischians. This is possibly due to variable ossification of cartilage that may have completed the perimeter of this foramen (Romer, 1927). However, only an open notch is currently known for Haya. The postpubic processes are thinner and longer than the prepubic process. They extend parallel to and beyond the distal end of the ischium to meet along the ventral midline below the position of the second caudal vertebra. The lateral surface of the distal three quarters of the postpubic process bears a shallow groove (Makovicky et al., 2011). This contrasts with Jeholosaurus where a groove is present only on the proximal quarter of the ventral surface (Han et al., 2012). The postpubic process is elliptical in cross section.

Ischium: The proximal ischium is divided into an iliac and a pubic peduncle. In IGM 100/2015 (fig. 38), these were damaged by insects or other scavengers (Makovicky et al., 2011). IGM 100/2019 provides additional information about their morphology. The iliac peduncle is anteroposteriorly broad, transversely compressed and probably squared off. It is slightly disarticulated, and therefore its original orientation relative to the ilium cannot be determined. The pubic peduncle is dorsoventrally expanded and is also transversely compressed and probably squared off, articulating with the pubis just distal to the probable position of the obturator notch in IGM 100/2015 (fig. 38). The pubic peduncle is longer than the iliac peduncle in IGM 100/2015, as in Jeholosaurus (Han et al., 2012). The two peduncles meet at an angle of approximately $140^{\circ}$ in lateral view. The broad, U-shaped concavity between them forms the ventral border of the acetabulum. The morphology of these peduncles differs little from other neornithischians (Norman et al., 2004). The remainder of the ischium constricts posteriorly to form a flattened shaft. This shaft twists approximately one third of the way along its length distal from the platelike proximal end, so that its medial surface faces 
dorsally (Makovicky et al., 2011). This twist is not as apparent in Orodromeus (Scheetz, 1999; D.E.B., personal obs. of MOR 294). Haya lacks the groove in this region that is variably present in Jeholosaurus and present in Eocursor (Butler, 2010; Han et al., 2012). Near the point at which the shaft twists, a tablike obturator process is present, as in the case for most early-diverging neornithischians and many ornithopods (Norman, 2004; Norman et al., 2004), but not Eocursor or Heterodontosaurus (Santa Luca, 1980; Butler, 2010). This process is placed slightly more distally in Haya than in Jeholosaurus (Han et al., 2012), but slightly more proximally than in Parksosaurus and Hypsilophodon (Parks, 1926; Galton, 1974a). The obturator process of IGM $100 / 2015$ is obscured by matrix along its articulation with the pubis; therefore, the morphology of the complete process remains unknown. Haya lacks the lateral groove just distal to the obturator process seen in Orodromeus (D.E.B., personal obs. of MOR 294). The shaft of the ischium is longer and straighter than that of Parksosaurus (Parks, 1926). The portion of the ischium distal to the obturator process progressively flattens dorsoventrally. A sharp ridge is present on its lateral surface, beginning at the point at which the shaft of the ischium twists (fig. 38) (Makovicky et al., 2011). This ridge possibly marks the origin for $\mathrm{m}$. puboischiofemoralis externus (Maidment and Barrett, 2011). The distal extent of the ischia relative to the postpubic shaft of the pubis cannot be determined in IGM 100/2015 due to damage. All visible surfaces of the ischia are smooth, without obvious muscle scars.

FEMUR: As described by Makovicky et al. (2011), the studied sample of Haya contains a growth series of femora. Two isolated femora, the $64 \mathrm{~mm}$ long (estimated) IGM 100/2020 (fig. 43) and the $162 \mathrm{~mm}$ long IGM 100/1324 (fig. 41), bracket the size range of currently known specimens. All of the other femora are intermediate in length between these, exemplified by the $129 \mathrm{~mm}$ long femur of IGM $100 / 2015$ (fig. 42) and the $125 \mathrm{~mm}$ long femur of IGM 100/2013 (fig. 44). The histology of some of these femora is discussed in a subsequent section of this paper.

The femoral head is offset from the neck by a distinct constriction. The head is approximately perpendicular to the shaft, and the neck angles dorsomedially away from the shaft at an approximately $135^{\circ}$ angle, similar to the angle in Koreanosaurus and Orodromeus (Scheetz, 1999; Huh et al., 2011). A wide rectangular groove for insertion of $\mathrm{m}$. iliotrochantericus (Maidment and Barrett, 2011) runs dorsolaterally along the posterior and medial faces of the head, as in Jeholosaurus, Orodromeus, Changchunsaurus, Koreanosaurus, and Hypsilophodon (Galton, 1974a; Scheetz, 1999; Butler et al., 2011; Huh et al., 2011; Han et al., 2012). A broad U-shaped valley separates the head from the greater trochanter proximally. A shallow circular depression occupies the lateral surface of the greater trochanter in IGM 100/1324 (fig. 41D), but this surface is flat in IGM 100/2013 (fig. 44D), IGM 100/2015 (fig. 42B), and IGM 100/2019 (fig. 34). The lateral surface of the greater trochanter is more extensively depressed in Changchunsaurus (Butler et al., 2011). In IGM 100/2013, there is a narrow, proximodistally oriented ridge on the posterior portion of the greater trochanter in lateral view that mirrors the lesser trochanter for a short distance (fig. 44D). This ridge is also present in Jeholosaurus (Han et al., 2012: fig. 10A, I). The lesser (or anterior) trochanter, while clearly distinct from the greater trochanter, is tightly appressed to its anterior surface, as in Heterodontosaurus, Fruitadens, and most neornithischians (Norman et al., 2004; Butler et al., 2012; Sereno, 2012; Galton, 2014). In contrast, the lesser trochanter is separated from the greater trochanter by a wide cleft in Lesothosaurus, Eocursor, Laquintasaura, Abrictosaurus, Agilisaurus, and Hexinlusaurus (He and Cai, 1984; Peng, 1992; Butler, 2010; Sereno, 2012; Barrett et al., 2014; Baron et al., 2017). The lesser trochanter is generally more anteroposteriorly expanded in these latter taxa than in Haya and others in which the lesser and greater trochanters are more tightly appressed. The proximal end of the lesser tro- 
chanter terminates distal to that of the greater trochanter, whereas in Changchunsaurus it extends proximally past the greater trochanter (Butler et al., 2011). The femur shaft bows anteriorly. Unlike in Orodromeus, which shows increased femoral curvature with increasing size (Scheetz, 1999), the smallest Haya femur (IGM $100 / 2020$ ) is curved to a similar degree as the larger femora. However, we note that no Haya femora as small as those of the smallest Orodromeus have yet been discovered. The femoral midshafts of IGM 100/2020, IGM 100/2015, and IGM 100/1324 are roughly circular in cross section, except toward the lateral side in the latter two specimens, on which the ridge of the linea intermuscularis caudalis (Maidment and Barrett, 2011) is developed (see subsequent discussion of the histology of these specimens). The linea intermuscularis cranialis is clearly visible on the anterior shafts of IGM 100/2013 and IGM $100 / 3672$ (figs. $15,44 \mathrm{~B}$ ). The fourth trochanter is located entirely on the proximal half of the femur as in Jeholosaurus, Orodromeus, and Hypsilophodon (Galton, 1974a; Scheetz, 1999; Han et al., 2012), whereas it arises more distally in Thescelosaurus (Gilmore, 1915). In Haya, the fourth trochanter is pendant and twists laterally about one third of the way from where it meets the shaft. The fourth trochanter twists more strongly than in Thescelosaurus (Gilmore, 1915), and is relatively thicker distally than in Koreanosaurus (Huh et al., 2011). The medial surface of its distal end contains a deep trough bounded by sharp ridges anteroventrally and posterodorsally. Another sharp ridge runs the length of the lateral surface of the fourth trochanter. The distal end of the fourth trochanter of IGM 100/2013 (fig. 44) is more transversely expanded than in IGM 100/2015 (fig. 42). The fourth trochanter of IGM $100 / 2013$ is more ventrally hooked than that of IGM 100/2015 and Jeholosaurus (Han et al., 2012). Scheetz (1999) also noted some intraspecific variation in fourth trochanter shape in Orodromeus. As in other ornithischians with a pendant fourth trochanter (Maidment and Barrett, 2011), caudofemoralis brevis likely inserted on the lateral side of the trochanter, and caudofemoralis longus inserted along the deep trough on the medial side. There is no obvious foramen lateral to the fourth trochanter in posterior view like in Jeholosaurus and Thescelosaurus (Gilmore, 1915; Han et al., 2012). There is a very shallow depression in this region on IGM 100/2013, but it is difficult to determine whether this is taphonomic in nature or is the trace of a foramen. An elliptical, roughened depression on the medial shaft just anterior to the fourth trochanter marks the insertion of $\mathrm{m}$. caudofemoralis longus, as in many ornithischians. The lateral condyle projects slightly further distally than the medial condyle. There is no anterior intercondylar sulcus, and the posterior intercondylar sulcus is deep, as is probably plesiomorphic for neornithischians (Norman et al., 2004). The medial condyle is larger than the lateral condyle in posterior view. The inflated medial condyle partially encloses the posterior intercondylar sulcus (figs. 42C, 43C, $44 \mathrm{~A}$ ), as in some elasmarians and iguanodontians (Salgado et al., 1997; Bell et al., 2019). A sulcus runs mediolaterally across the distal surface of the lateral condyle.

TiвiA: The proximal end of the tibia is anteroposteriorly expanded and crescent shaped in proximal view, as the cnemial crest and inner condyle form a medially bowing arc with their apices pointing laterally toward the fibula (fig. 45). The fibular condyle is separated from these by deep sulci (the anterior sulcus is the incisura tibialis) and points laterally to articulate with the fibula. The fibular condyle is smaller in Haya than in Jeholosaurus (Han et al., 2012). As in Orodromeus, Changchunsaurus, Koreanosaurus, and Hypsilophodon (Galton, 1974a; Scheetz, 1999; Huh et al., 2011), but not Hexinlusaurus, "Yandusaurus" hongheensis, Jeholosaurus, Anabisetia, and Gasparinisaura (He and Cai, 1984; Coria and Salgado, 1996; Salgado et al., 1997; Coria et al., 2007; Han et al., 2012), there is a small anterolaterally directed accessory condyle separated posteriorly from the fibular condyle by a broad, shallow sulcus. A poorly developed accessory condyle is present in Lesothosaurus 
and Eocursor (Thulborn, 1972; Butler, 2010). The accessory condyle is broader and more rounded in Changchunsaurus and Orodromeus than in Haya (Scheetz, 1999; Butler et al., 2011). In IGM $100 / 2013$, the medial surface of the cnemial crest contains a deep fossa with a raised anterior rim and a striated floor (fig. 47A, B). This fossa is not as well defined in IGM 100/2015. The expanded proximal end tapers gradually to the shaft, which is roughly circular at its midpoint, but distal to that point it expands mediolaterally. The shaft is largely straight and smooth. In IGM 100/2013, the posteromedial surface of the shaft has a low mound of unknown identity just distal to its midpoint and adjacent to the fibular eminence (fig. 47A, C). The fibular eminence, sensu Han et al. (2012), is a low, laterally convex ridge on the distal half of the lateral side of the tibia (figs. 46A, B, 47C), similar in shape to that of Jeholosaurus and Albertadromeus (Han et al., 2012; Brown et al., 2013). Proximal to the fibular eminence, the tibia shaft is deflected away from the fibula; distally, the fibula and tibia are tightly appressed. The mediolateral expansion of the distal shaft forms the medial and lateral malleoli. The lateral malleolus projects further distally than the medial malleolus (Makovicky et al., 2011). The medial malleolus is more anteroposteriorly expanded than the lateral malleolus. There is a deep depression between the malleoli anteriorly, and a shallow depression between them posteriorly. As noted by Makovicky et al. (2011), Haya lacks the ridge between the two malleoli posteriorly that is present in Gasparinisaura and Diluvicursor (Coria and Salgado, 1996; Herne et al., 2018).

Fibula: The fibula is not fused to the tibia, as in almost all neornithischians, but in contrast to Heterodontosaurus and Albertadromeus, in which these two elements are fused distally (Santa Luca, 1980; Brown et al. 2013). The proximal end of the fibula is anteroposteriorly expanded. The bone tapers distally and is narrow for much of its length, but it expands anteroposteriorly again just proximal to its articulation with the calcaneum. The fibula shafts of Koreanosaurus and
Thescelosaurus appear to be proportionally thicker for much of their length than in Haya (Galton, 1974b; Huh et al., 2011). The medial surface of the proximal fibula of IGM 100/2013 is concave and has a deep fossa, possibly for articulation with the fibular condyle of the tibia. Approximately one third of the way down the shaft, at its most laterally swollen point, the fibula has a rugose patch for muscle attachment (fig. 47C, D). In anterior view, the proximal half of the fibula bows laterally, whereas the distal half is more tightly appressed to the fibula. A thin, medially directed flange from the fibula overlaps the tibia near its midpoint. This flange is slightly shorter proximodistally and more rectangular in Orodromeus (Scheetz, 1999) and absent in Jeholosaurus (Han et al., 2012). The distal end of the fibula is rounded in proximal view. In lateral view, a triangular projection from the fibula inserts between the tibia and calcaneum.

TARSALS: The astragalus and calcaneum are tightly appressed in all Haya specimens (figs. 46, 47). Their distal articular surfaces merge smoothly (Makovicky et al., 2011). They may or may not be partially fused, as only short traces of a suture between the two elements remain. All available Haya astragali and calcanea are articulated with the tibia and fibula, preventing description of their proximal surfaces. They are not fused to the tibia or fibula, in contrast to Heterodontosaurus, in which the tibia, fibula, astragalus, and calcaneum are all fused to one another (Santa Luca, 1980). The astragalus is crescentic in medial view, forming a smoothly rounded articular surface. Anteriorly, the astragalus gives rise to a proximodistally short, fingerlike ascending process. The ascending process is longest and most transversely expanded in IGM 100/2015. In IGM 100/2013 and IGM 100/3178 (figs. 46, 47), it is more spikelike, as in Hypsilophodon (Galton, 1974a). Orodromeus differs from Haya in having a forked ascending process (Scheetz, 1999). In Haya, two depressions lie to either side of the base of the ascending process, and a transverse groove cuts across the astragalus distal to them 
(Makovicky et al., 2011). In distal view, the astragalus narrows gradually in anteroposterior width to its articulation with the calcaneum. The calcaneum is ovoid in lateral view, with a large pit occupying the center of its lateral surface. Small portions of both the calcaneum and astragalus are visible in posterior view.

Two distal tarsals are present, as in most neornithischians (Norman et al., 2004). They consist of a discoid medial tarsal and a blocky lateral tarsal (fig. 47B). The medial element may comprise fused distal tarsals two and three, given its articulation with the second and third metatarsals. The cuboidal lateral distal tarsal articulates solely with the fourth metatarsal. Alternatively, some of the plesiomorphic four distal tarsals may have been lost from these positions during neornithischian evolution, with adjacent tarsals shifting to take their places. Many authors (e.g., Han et al., 2012; Santa Luca, 1980) interpret the medialmost distal carpal as distal carpal 1, despite its articulation with metatarsal II. This is plausible, but it is also possible that distal tarsal 1 was lost or fused onto distal tarsal 2 when the proximal articular surface of metatarsal I became reduced in size. Therefore, in taxa with three distal tarsals (e.g., Heterodontosaurus) these may be distal carpals 1, 2, and 3, or they may be distal carpals 2, 3, and 4. Likewise, in taxa with two distal tarsals (e.g., Haya), they may be distal tarsals $1+2$ and 3 or $2+3$ and 4. We consider both equally likely, and in the absence of further evidence, see no a priori reason to identify the distal tarsals as 1, 2, and 3 . Given this uncertainty as to their identities, we refer to the elements in Haya simply as the medial and lateral distal tarsals. In light of this, the distal tarsals of Orodromeus and Jeholosaurus do not seem to be as unusual as described by Han et al. (2012), as the morphologies of the medial and lateral distal tarsals are very similar to those of Haya (IMG 100/2013, fig. 47B). The lateral distal tarsal of Haya is similar to the lateralmost distal tarsal of Heterodontosaurus, which is cuboidal with a possible foramen on its posterior (plantar) surface (Santa Luca, 1980).
Metatarsals: IGM 100/2013 and IGM 100/3178 have complete, articulated metatarsals and phalanges (figs. 47, 48). The overall proportions and morphology of the metatarsals are very similar to other early-diverging neornithischians (Norman et al., 2004). One exception is Thescelsosaurus, in which the metatarsals are shorter relative to the phalanges than in taxa such as Haya and Hypsilophodon (Gilmore, 1915; Galton, 1974b). In Haya, metatarsal I is splintlike, with a mediolaterally compressed proximal shaft that expands anteroposteriorly and transversely toward its distal end. Metatarsal I articulates with the ankle at the same proximal level as metatarsal II. Metatarsal I has a simple, rounded distal articular surface. Metatarsals II and IV are subequal in length, with ginglymoid distal articular surfaces and collateral ligament pits. Metatarsal II is parallel and tightly appressed to metatarsal III for most of its length, whereas metatarsal IV bends laterally away from III. Metatarsal III is the longest metatarsal and has the most rounded and well-separated distal condyles. Its distal half bends slightly laterally. Metatarsal III also has the deepest collateral ligament pits of all the metatarsals of IGM 100/3178. The posterior surface of metatarsal II bears a longitudinal ridge, whereas that of metatarsal III is flat (Makovicky et al., 2011). The ventral surface of metatarsal IV has a diagonal ridge that runs from the medial surface proximally to the lateral surface distally, merging with the ventral edge of the lateral condyle, as in Jeholosaurus, Changchunsaurus, and Hypsilophodon (Galton, 1974a; Butler et al., 2011; Han et al., 2012). Metatarsal V is an anteroposteriorly flat, rectangular element that lacks clearly defined articular surfaces. It is more transversely expanded than the rodlike fifth metatarsal of Hypsilophodon (Galton, 1974a). The distal ends of the metatarsals are convex dorsally and lack any extensor pits.

Pedal Phalanges: The pedal phalangeal formula of Haya is 2-3-4-5-0, as in nearly all earlydiverging neornithischians (Norman et al., 2004) (figs. 47, 48). As is typical for these taxa, the first phalanges on all the digits are the longest. Each 
phalanx is smooth dorsally and ventrally. All phalanges except I-1, II-1, and IV-1 have a dorsal lip that articulates with the extensor groove of the preceding phalanx. There is a short dorsal lip on phalanx III-1 in one specimen of Haya, IGM 100/3178 (fig. 48B, C), but not in another, larger specimen, IGM 100/2013 (fig. 47). The presence of a short dorsal lip on phalanx III-1 is also variable in Jeholosaurus, but in that taxon the larger specimen (IVPP V15939) has the lip, while the smaller one (IVPP V12529) lacks it (Han et al., 2012: fig. 12A, B, E, F). Therefore, in contrast to the observation by Makovicky et al. (2011) and the condition in Hypsilophodon (Galton, 1974a), Haya does not always lack dorsal lips on all proximal phalanges. All nonungual phalanges are ginglymoid and bear deep collateral ligament pits. Phalanx I-1 is relatively long, as in Changchunsaurus, whereas it is proportionately shorter in Jeholosaurus (Butler et al., 2011; Han et al., 2012). The terminal phalanx of each digit is a clawlike curved ungual. The digit I ungual is the narrowest, whereas the others are more transversely expanded. The ungual on digit I is also proportionately narrower than that of Jeholosaurus (Han et al., 2012). The ungual on digit III is the longest, as in Jeholosaurus but in contrast to Changchunsaurus, in which the digit II claw is the longest (Butler et al., 2011; Han et al., 2012). The overall pedal phalangeal morphologies and proportions of Othnielosaurus (YPM 57181: a cast of BYU 163) and Hypsilophodon are also very similar to those of Haya (Galton and Jensen, 1973; Galton, 1974a). In contrast, the pedal phalanges of Thescelosaurus (Gilmore, 1915) are more transversely expanded and the unguals are less curved and pointed than those of Haya, creating an overall impression of greater robustness.

\section{GASTROLITHS}

Gastroliths are associated with IGM 100/2015, IGM 100/3661, IGM 100/3672, and IGM $100 / 3182$ (figs. $14,15,27,49,50$ ). In the latter two specimens, they are preserved near the abdominal cavity, probably only slightly dis- lodged from their life position inside the animal. They underlie the tibia and fibula in IGM 100/2015 (figs. 27, 49). The lithology of the gastroliths is varied within all specimens, but is probably primarily quartz and chert (Makovicky et al., 2011). The pebbles are smooth, tightly grouped, and have rounded edges. The overall volume of gastroliths is roughly consistent among the specimens and was estimated at 92.7 mL by Makovicky et al. (2011) for IGM 100/2015. The gastroliths associated with IGM 100/2015 range from approximately $5-13 \mathrm{~mm}$ in maximum diameter, and from $6-15 \mathrm{~mm}$ in maximum diameter for those with IGM 100/3182. The upper values are presumably a rough minimum value for the fully distended diameter of the esophagus. As previously noted by Makovicky et al. (2011), clasts of this size are generally absent from the matrix surrounding the Haya specimens. Similar clasts are abundant on the surfaces of Javkhlant Formation exposures at the Khugenetslavkant locality. However, these clasts are absent in pits dug into the outcrop, suggesting that they may be primarily derived from weathering of abundant Haya griva skeletons at the locality over many years (M.A.N., personal obs.).

\section{HISTOLOGY}

The histology of early-diverging neornithischians and iguanodonts has become increasingly well studied in recent years (Chinsamy, 1995; Chinsamy et al., 1998; Horner et al., 2009; Woodward et al., 2011, 2018; Cerda and Chinsamy, 2012; Hübner, 2012; Werning, 2012; Barrett et al., 2014), but many key smaller-bodied "hypsilophodontid" taxa remain unsampled. As in other dinosaur groups (Erickson, 2005), this grade exhibits a general trend for slower growth rates in small-bodied taxa than in large-bodied taxa, and few skeletally mature individuals are known (Horner et al., 2009). North American and Australian taxa primarily form the basis for these studies, with only one recent description of the histology of an Asian basal neornithischian published while this paper underwent final revi- 
A
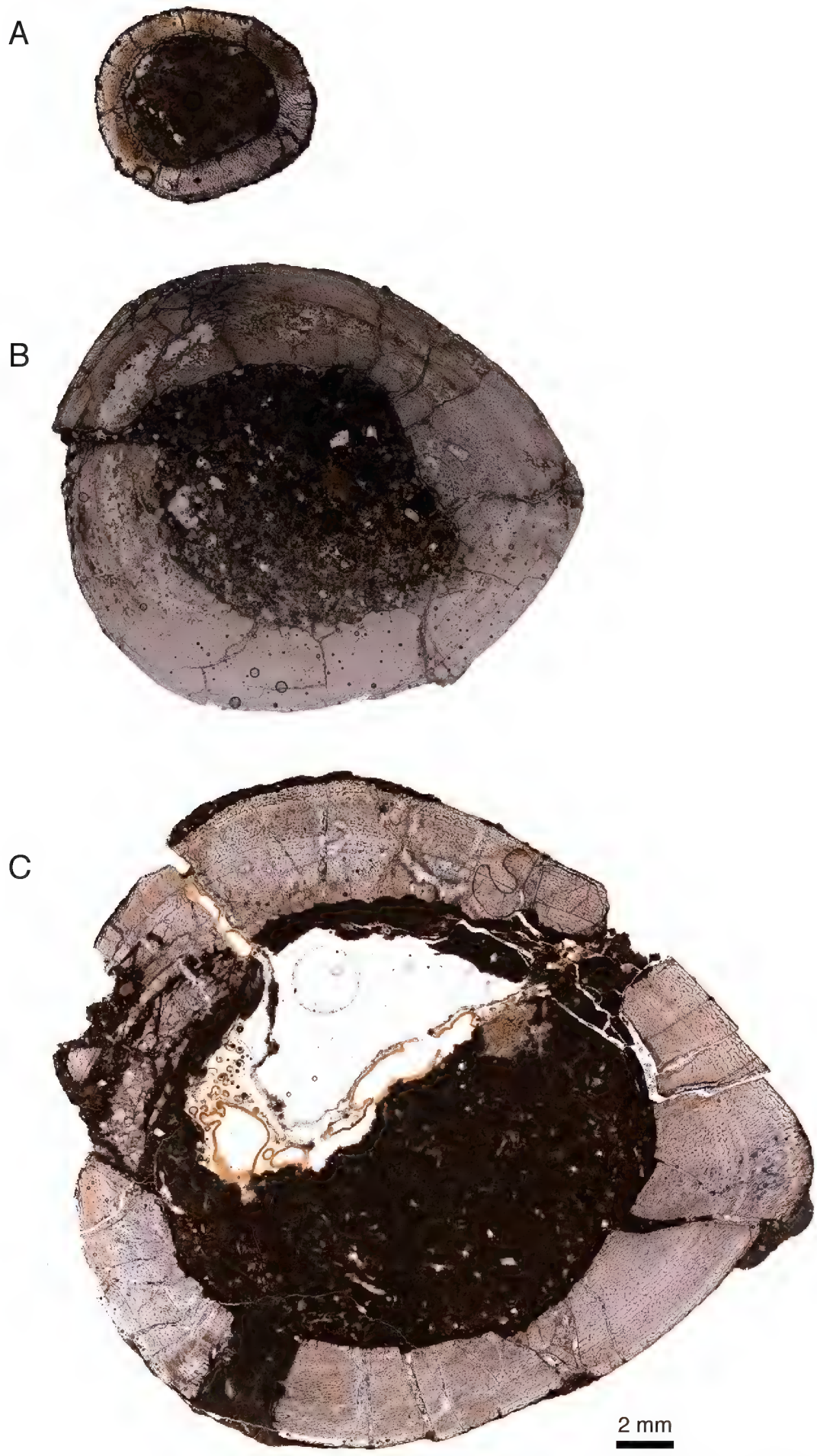

FIG. 51. Transverse thin sections of Haya griva femora. A, IGM 100/2020 , B, IGM 100/2015, and A, IGM 100/1324 under plane polarized light. 
sion (Han et al., 2020). As for other fossil vertebrates, the ontogenetic stage of specimens may impact their phylogenetic placement (e.g., Kammerer, 2011; Tsuihiji et al., 2011; Campione et al., 2013). In order to determine the absolute ages of the Haya griva specimens to describe their growth patterns, compare them with other dinosaurs, and place their sizes and morphologies in an ontogenetic context, we sampled four Haya griva femora from the Javkhlant Formation at the Khugenetslavkant locality for histological analysis. These specimens span the entire known size range of Haya specimens, from the smallest, IGM $100 / 2020$, to a midsize specimen, IGM $100 / 2015$, to the largest, IGM 100/1324.

Prior to sampling the specimens, IGM 100/2020, IGM 100/2015, and IGM 100/1324 were scanned using a Space Spider surface scanner (Artec, Luxemborg) and 3D printed to preserve a record of their complete morphology. A cast was made of the fourth intermediate-sized specimen, IGM 100/3672. Stereolithography (STL) files of the specimens are available as part of the supplementary information of this paper (https://doi.org/10.5531/sd.sp.47). After this, a 5-8 $\mathrm{mm}$ thick midshaft segment just distal to the fourth trochanter was removed from each femur. The segments were embedded in Epo-tek 301-2 epoxy and transversely sectioned into $1.5 \mathrm{~mm}$ thick wafers with a Buehler Isomet 1000 precision saw. The wafers were mounted with Epo-tek 301-2 epoxy on prefrosted standard $(27 \times 46$ $\mathrm{mm}$ ) glass petrographic slides, and ground until transparent. The slides were immersed in mineral oil before viewing with a Leitz Laborlux 11 Pol S petrographic microscope. In order to photograph the entire cross sections of the femora, we used a Zeiss Axio Imager 72 Automated Microscope Imaging System, which consists of a petrographic microscope with an automated, mobile stage for image stitching. The description primarily uses standard histological terms from Francillon-Vieillot et al. (1990). Femur circumferences were measured with software (Image), NIH, Bethesda, Maryland) from photomicrographs of IGM 100/2020, IGM 100/2015, IGM
$100 / 1324$, and from a photograph of a thin section of IGM 100/3672. Body mass was estimated for the four specimens by entering the thin-section femoral circumference measurements into the corrected quadrupedal mass estimation equation for bipeds implemented in the R software package MASSTIMATE (Campione, 2013; Campione et al., 2014). We also estimated body masses using the developmental mass extrapolation (DME) method of Erickson and Tumanova (2000), given that it may be more suitable for intraspecific mass estimation. The masses obtained from both methods were nearly the same, except in the case of IGM 100/3672, which has a narrower circumference than would be predicted for its length using DME. Therefore, we use the MASSTIMATE values for all subsequent discussion.

IGM 100/2020 is an isolated right femur with an estimated length of $63.9 \mathrm{~mm}$ (figs. 43, 51A, $52 \mathrm{~A}$ ). This value was estimated by measuring the distance between the proximalmost extent of the fourth trochanter and the distalmost point of the medial condyle on the complete femora of IGM $100 / 2015$ and IGM 100/1324. We then divided this length by the complete femur length of those specimens to obtain an average ratio of 1.4. We then multiplied this ratio by the preserved fourth trochanter to medial condyle distance of IGM $100 / 2020$ to obtain the estimated complete length for this specimen, assuming isometry in these femur proportions through ontogeny. This is a reasonable assumption given that the ratios in both IGM 100/2015 and IGM 100/1324 are nearly 1.4 , and given that the femur has been shown to grow isometrically in the related taxa Tenontosaurus and Dryosaurus (Kilbourne and Makovicky, 2010). An estimated femur length of $63.9 \mathrm{~mm}$ also falls within the confidence interval of the linear scaling model for ornithopod femur length developed by Kitchener et al. (2019), given that the anteroposterior diameter of IGM $100 / 2020$ is $7 \mathrm{~mm}$.

IGM 100/2020, the smallest available femur, is subcircular in midshaft cross section, with a slight elongation toward the lateral side. It con- 


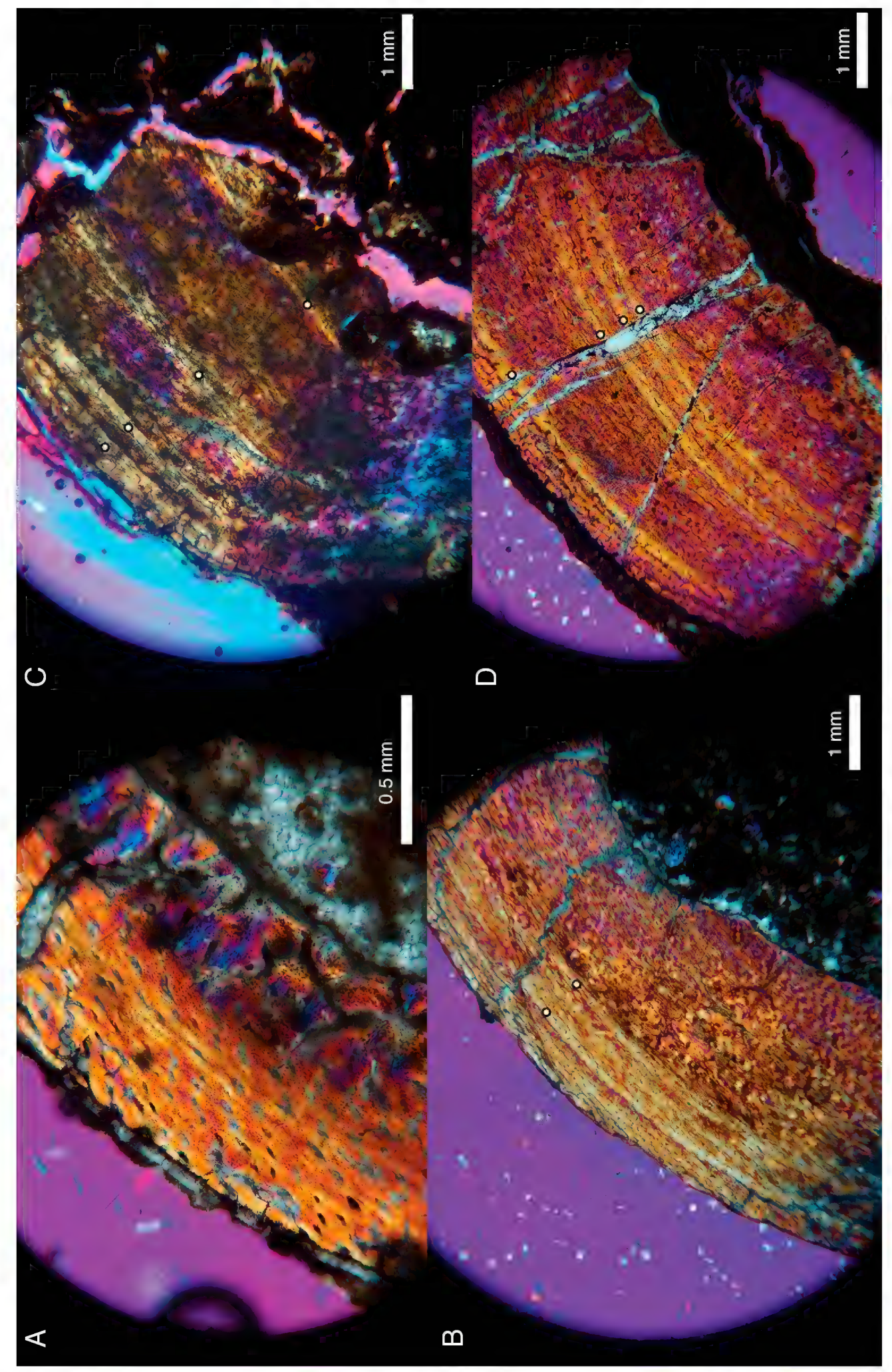

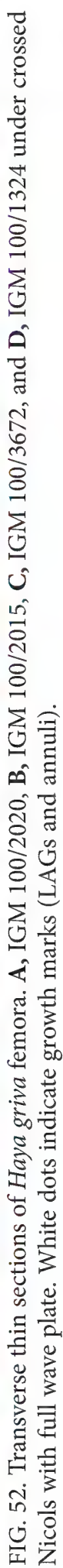


sists of primarily woven-fibered bone (figs. 51A, 52A). Parallel-fibered bone predominates in some places, especially in the outer half of the cortex on the medial side. A similar transition from woven-fibered to parallel-fibered bone is seen in a similar-sized Australian Cretaceous ornithopod femur of comparable length (Woodward et al., 2018: fig. 3b). Similar transitions from less to more organized bone tissue have been likened to the "hatching lines" or "natal lines" present in extant vertebrates (e.g., Castanet, 1986; Castanet et al., 2004; Curtin et al., 2012), and are interpreted to mark a slowdown in growth immediately following birth or hatching (Rogers et al., 2016; Woodward et al., 2018). There are many large longitudinal vascular canals. Osteonal lamellar bone is not well developed inside these canals, and many of them are open at the periosteal surface. The vascularization changes to radial near the lateral side of the bone and reticular near the posterior side. Trabeculae are present on the inner circumference of the femur on the medial side, but not on the lateral side. These differences possibly reflect lateral migration of the medullary cavity as the bone grew. There is no inner circumferential layer of lamellar bone. Osteocyte density varies, especially on the medial side, where it is higher toward the medullary cavity and patchy in the remainder of the cortex. No growth marks are preserved. Overall, the histology is very similar to that of perinatal Australian ornithopod, Orodromeus, Tenontosaurus, and Dryosaurus femora and tibiae (Horner et al., 2009; Werning, 2012; Woodward et al., 2018), as well as a very young Romanian Late Cretaceous ornithopod tibia (Brusatte et al., 2013). The large size and high density of the vascular canals, lack of extensive osteonal lamellar bone in the vascular canals, lack of erosion from the inner circumference, lack of growth marks, the bone-fiber types and their organization, and the possible presence of a hatching line all suggest that IGM $100 / 2020$ was a relatively rapidly growing perinatal, possibly hatchling, individual at its time of death.
IGM 100/2015 is the right femur from an articulated postcranial skeleton (figs. 27, 42, 51B, 52B). It measures $128.9 \mathrm{~mm}$ long. Its midshaft cross section is elliptical, except underneath the linea intermuscularis caudalis (Maidment and Barrett, 2011) laterally, where the sides of the femur come together at a sharper point. Bone is missing along the inside of the whole medullary cavity circumference, though less extensively from the lateral side. This erosion is likely in part a taphonomic modification. The bone is primarily woven fibered near the inner cortex and parallel fibered surrounding the growth marks in the outer $1 / 3$ of the cortex (some woven bone is interspersed between these bands) (fig. 52B). The section contains primarily longitudinal vascular canals, with some anastomoses stretching across two to five canals. There is some lamellar bone encircling the canals to form primary osteons. Osteocyte density is high and more regular than that of IGM 100/2020. One line of arrested growth (LAG) and two annuli are present, with the annuli occurring outside the LAG toward the external surface. The outermost annulus grades into a LAG along the posterior side of the femur, resulting in two clear LAGs visible along this portion of the section. The growth marks cannot be traced completely around the circumference, because of cracks and possible erosion of portions of the cortex near the periosteal surface. The inner annulus can be traced only a short distance, and so may not represent a true annual growth mark. Nevertheless, given the current information, we are reasonably confident that at least the innermost and outermost growth marks would have encircled the femur before taphonomic modification. Therefore IGM 100/2015 likely died, at minimum, in its third year of life. No secondary osteons are present. The presence of growth marks, relatively smaller vascular canals, moderate primary osteonal development, and resorption along the medullary cavity suggest a relatively advanced ontogenetic age compared to IGM 100/2020, and the overall histology is comparable to hypothesized juvenile or subadult femora of Australian "hypsilophodontids," Orodromeus, and Tenontosaurus (Horner et 
al., 2009; Werning, 2012; Woodward et al., 2018). Additionally, the histological features of this femur correspond closely to Hübner's (2012) Stage 3 or Late Juvenile Stage Dysalotosaurus femora.

IGM $100 / 3672$ is a femur from a partial postcranial skeleton (fig. 15). It measures $157 \mathrm{~mm}$ long. The midshaft cross section is crushed, but is broadly similar in shape to those of IGM $100 / 2015$ and IGM 100/1324. Much of the bone microstructure and vascular pattern is obscured by possible postmortem fungal alteration, but the less altered patches show parallel- to wovenfibered bone typical of IGM 100/2015 and IGM 100/1324 (fig. 52C). Four annuli consisting of narrow bands of parallel-fibered bone are visible through portions of the cortex (fig. 52C). At least one annulus may contain a LAG.

IGM $100 / 1324$ is an isolated left femur that is $162 \mathrm{~mm}$ long (figs. 41, 51C, 52D). Its midshaft cross section is similar in outline to that of IGM $100 / 2015$, with the ridge of the linea intermuscularis caudalis on its lateral side. The outermost vascular canals in the area beneath the ridge are radial, and this area corresponds to the "posterolateral plug" described by Hübner (2012) as a site where muscle forces disrupt the organization of the surrounding bone. The medullary cavity is largely eroded. The bone is primarily parallel fibered with some woven-fibered bone interspersed, particularly between the bands of parallel-fibered bone that surround the growth marks (fig. 52D), as in IGM 100/2015. Proceeding outward from the medullary cavity, the vasculature transitions from longitudinal with some anastomoses to extensively reticular. Many vascular canals are open at the external surface. Some primary osteons exhibit more extensive infilling with lamellar bone than either IGM 100/2020 or IGM 100/2015. Osteocyte density is high and regularly distributed. Four annuli that grade into LAGs along the lateral side of the femur are present. As in IGM 100/2015, they can be traced for about three quarters of the femoral circumference due to erosion of some areas. Three of the growth marks are closely spaced in the inner half of the cortex, whereas the fourth is more widely separated from these and lies toward the outer cortex. Based on the number of growth marks, moderately high level of osteonal development, and organization of bone tissue types, IGM 100/1324 likely belonged to a subadult individual, probably of an older ontogenetic age than IGM 100/2015.

None of the sampled Haya specimens exhibit an external fundamental system (EFS), a series of closely spaced LAGs near the periosteal surface (figs. 51, 52). Growth mark spacing shows no sign of decreasing toward the outer surface in any specimen. No decrease in vascularity is apparent toward the outer cortex, and indeed the vascularity becomes extensively reticular near the periosteal surface in IGM 100/1324 (fig. 52D), suggesting a high growth rate was maintained (Castanet et al., 1996, 2000). Based on these observations, all sampled Haya specimens appear to have been actively growing at their times of death.

Because the transverse section of IGM $100 / 2015$ fits completely within the medullary cavity of IGM $100 / 1324$ (fig. 51), it is possible to estimate the number of growth marks lost to medullary cavity expansion in the larger femur by adding the number of growth marks from the smaller. IGM 100/3672 overlaps both IGM $100 / 2015$ and IGM 100/1324, with possibly one growth mark from IGM 100/2015 equivalent to the innermost growth mark of IGM 100/3672. This retrocalculation method suggests that IGM $100 / 3672$ may have lived to five years of age, and IGM 100/1324 to at least six years of age. It is not possible to easily determine how many growth marks, if any were lost to erosion in IGM 100/2015. Therefore the two growth marks of IGM 100/2015, the estimated five growth marks of IGM 100/3672, and the estimated six growth marks of IGM 100/1324 are considered minimum numbers. We acknowledge one limitation of this section stacking technique is that it assumes no intraspecific variation in size at a given age (Erickson, 2014). Given that we cannot measure the complete circumferences of all LAGs, we cannot use the graphical retrocalcula- 


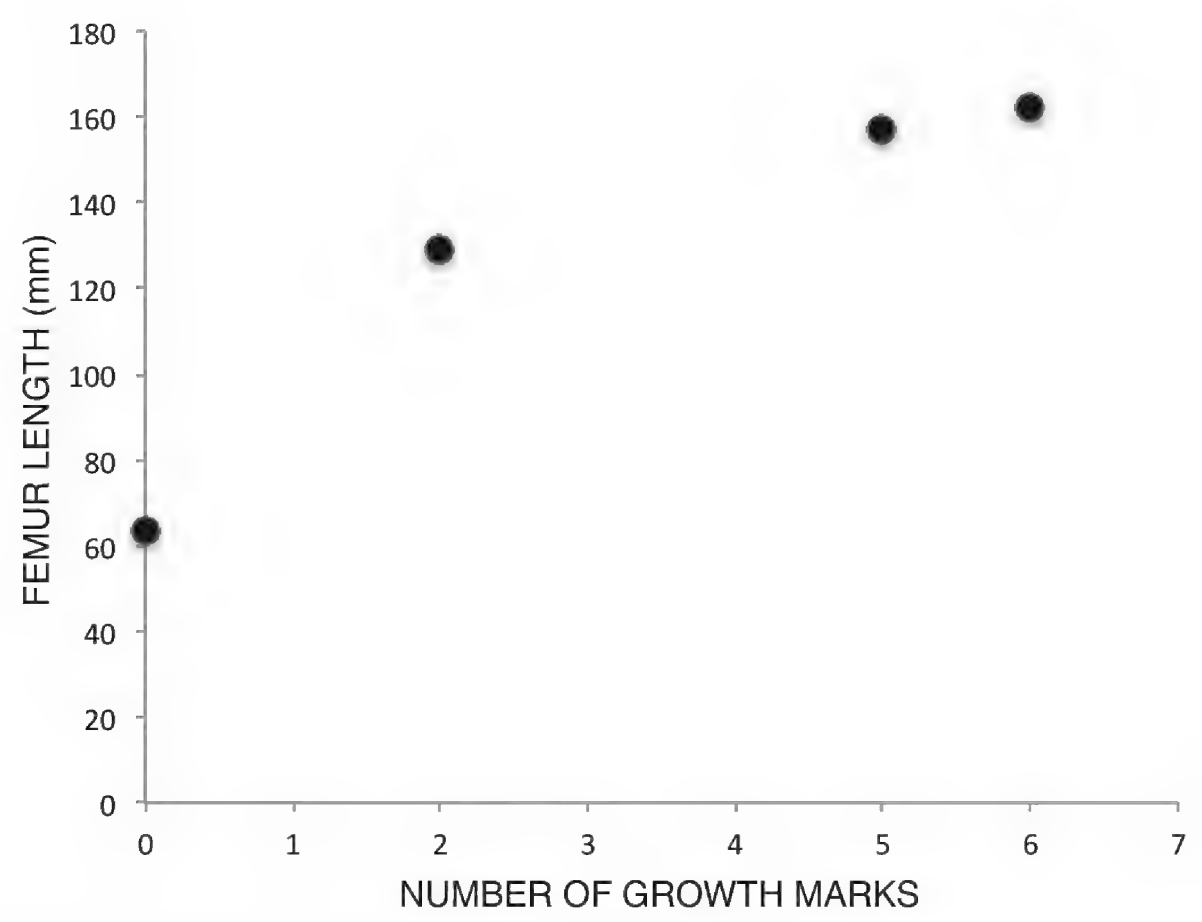

FIG. 53. Graph of age (as determined by number of growth marks) vs. femur length in Haya griva.

tion methods of Bybee et al. (2006) or Cooper et al. (2008), though these may produce a more accurate estimate of the number of missing growth marks. Likewise, because growth-line spacing varies greatly within each specimen, and the spacing between LAGs is not always constant within a given growth line interval, it is also difficult to extrapolate the number of LAGs from the thickness of preserved LAG intervals (e.g., Horner and Padian, 2004). Additionally, the close spacing of the first two growth marks in IGM 100/2015 cannot be assumed to typify early growth. Therefore, section stacking, despite its limitations, is the best option for retrocalculating growth marks in these specimens at this time.

Plotting femur length against growth mark count reveals an increasing trend with age (fig. 53). With only four data points, we cannot fit a curve to these data, though it is clear based on growth mark spacing that none of the Haya specimens had yet reached the growth plateau that indicates skeletal senescence. Plotting femur circumference and estimated body masses against age reveals a similar pattern (figs. 54, 55). Finally, given that the largest sampled femur was actively growing at the time of death, our histological analysis implies that none of the Haya specimens discovered so far are likely to be skeletally mature (i.e., possess an EFS). The vast majority of specimens recovered are of animals similar in size and possibly age to IGM 100/2015, a possible late juvenile specimen. Our analysis also reveals that the longevity, final body size and morphology at skeletal maturity of Haya all remain unknown, as is the case for many other dinosaurs (e.g., Erickson, 2005, 2014; Horner et al., 2009). While the analyzed Haya specimens are probably not all from a single population, the fact that nearly all known specimens are skeletally immature individuals is consistent with the high juvenile mortality and right-skewed age distributions of other ornithischians (Andrzejewski et al., 2019; Erickson et al., 2009; Woodward et al., 2015). This may be typical for nonavian dinosaurs in general (Erickson et al., 2009). 


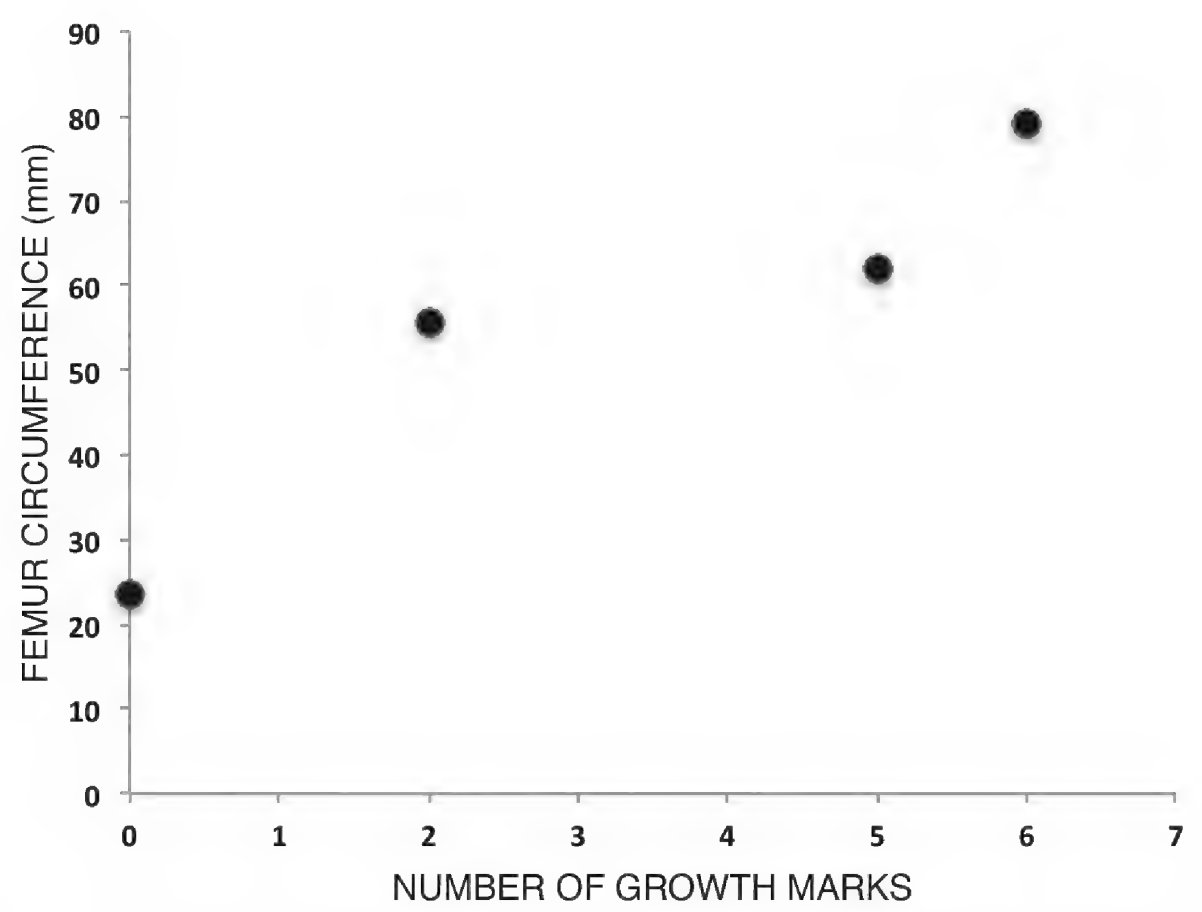

FIG. 54. Graph of age (as determined by number of growth marks) vs. femur circumference in Haya griva.

\section{PHYLOGENY}

In order to assess the monophyly of specimens assigned to Haya griva and their phylogenetic position(s), taking into account the impact of the new anatomical information gleaned in the course of this study, and also account for the impact of ontogenetically variable characters and other polymorphism on the phylogeny, we performed three analyses. All analyses made use of the data matrix of Madzia et al. (2018), which utilizes the same 255 characters as Boyd (2015), with an expanded taxon matrix of 75 taxa. Our scoring of Haya differed in several respects from Madzia et al. (2018). The Nexus files in the supplementary information (https://doi.org/10.5531/sd.sp.47) contain all character scorings that were changed from the original analysis. All characters were treated as equally weighted and unordered. Marasuchus was used as the outgroup, in accordance with previous analyses of this data matrix. All analyses were performed in TNT v. 1.5 (Goloboff et al., 2008; Goloboff and Catalano, 2016).
Analysis 1: This analysis included nine key Haya specimens, chosen for their completeness, and to include those with both cranial and postcranial material. This is important to assess whether or not those specimens consisting solely of either a skull or postcrania are part of the same clade. We ran the analysis for 10,000 random addition sequences, using the tree-bisection reconnection algorithm (TBR) for branch swapping. Ten trees were held for each replication. After this, a subsequent round of branch swapping was performed using the bbreak command in TNT (Goloboff et al., 2008). Bootstrapping (with 10,000 replicates) and Bremer support analyses were utilized to assess branch support.

We recovered 100,000 trees of 927 steps (CI: 0.335 , RI: 0.646 ), hitting the limit of tree storage we defined. Nevertheless, we likely sampled tree space adequately because the strict consensus resulting from this analysis is identical to one produced by an analysis in which 1,000,000 trees of 927 steps were saved. Therefore we followed the same search protocols $(10,000$ replications, 


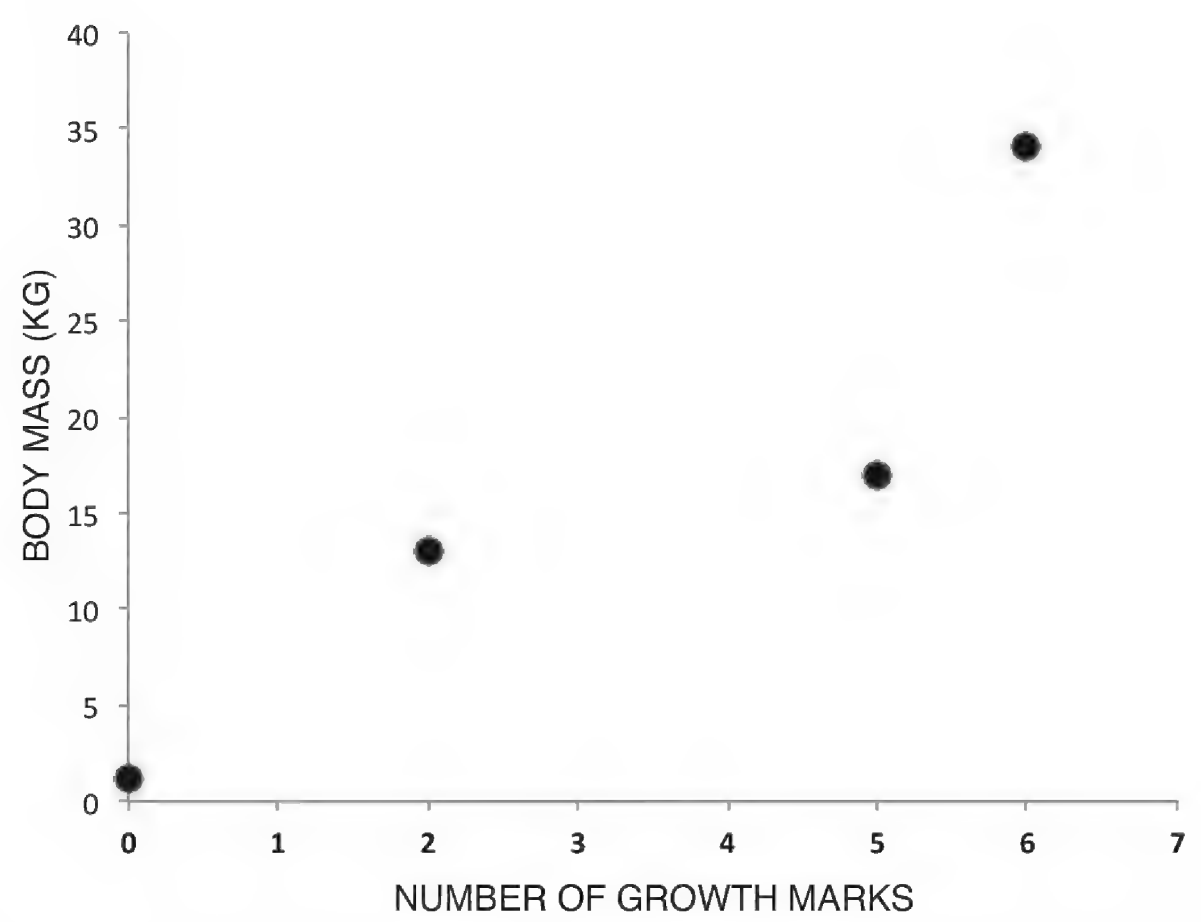

FIG. 55. Graph of age (as determined by number of growth marks) vs. body mass (as calculated from femur circumference) in Haya griva.

holding 10 trees for each) for all analyses. In agreement with Madzia et al. (2018), the prunnelsen command in TNT revealed that the resolution of the strict consensus tree could be improved by pruning two taxa a posteriori, Leallynasaura and Morrosaurus, as the positions of these taxa were highly labile, creating a lack of resolution in the strict consensus tree.

Much of Neornithischia is unresolved on the strict consensus tree. However, Thescelosauridae is resolved, and includes all Haya specimens. The Haya specimens do not form a clade to the exclusion of all other thescelosaurid taxa. The pruned analysis shows increased resolution near the base of Neornithischia, but all of Thescelosauridae, including all of the Haya specimens, remains unresolved (fig. 56). Clades less inclusive than Ornithischia have very low Bremer support and bootstrap values, typically 1 and less than 50, respectively. Pruning Leallynasaura and Morrosaurus does not appreciably improve these support values.
AnAlysis 2: This analysis was run with the same parameters as Analysis 1 above, with the exception that all Haya specimens were combined into a single composite operational taxonomic unit (OTU), with characters that varied between individual specimens coded as polymorphic.

We recovered 100,000 trees of 916 steps (CI: 0.340, RI: 0.635), hitting the limit of tree storage we defined. After examining the strict consensus tree, Leallynasaura and Morrosaurus were then pruned a posteriori, as in Analysis 1.

On the strict consensus tree, the composite Haya was recovered in a polytomy with Thescelosaurinae and Orodrominae. Following pruning of Leallynasaura and Morrosaurus, the topology is more resolved near the base of Neornithischia, as in Analysis 1, and Haya still forms a polytomy with Thescelosaurinae and Orodrominae (fig. 57). As for Analysis 1, clades less inclusive than Ornithischia have very low Bremer support and bootstrap values, typically 1 and less than 50, respectively. Pruning Leallynasaura and Morro- 


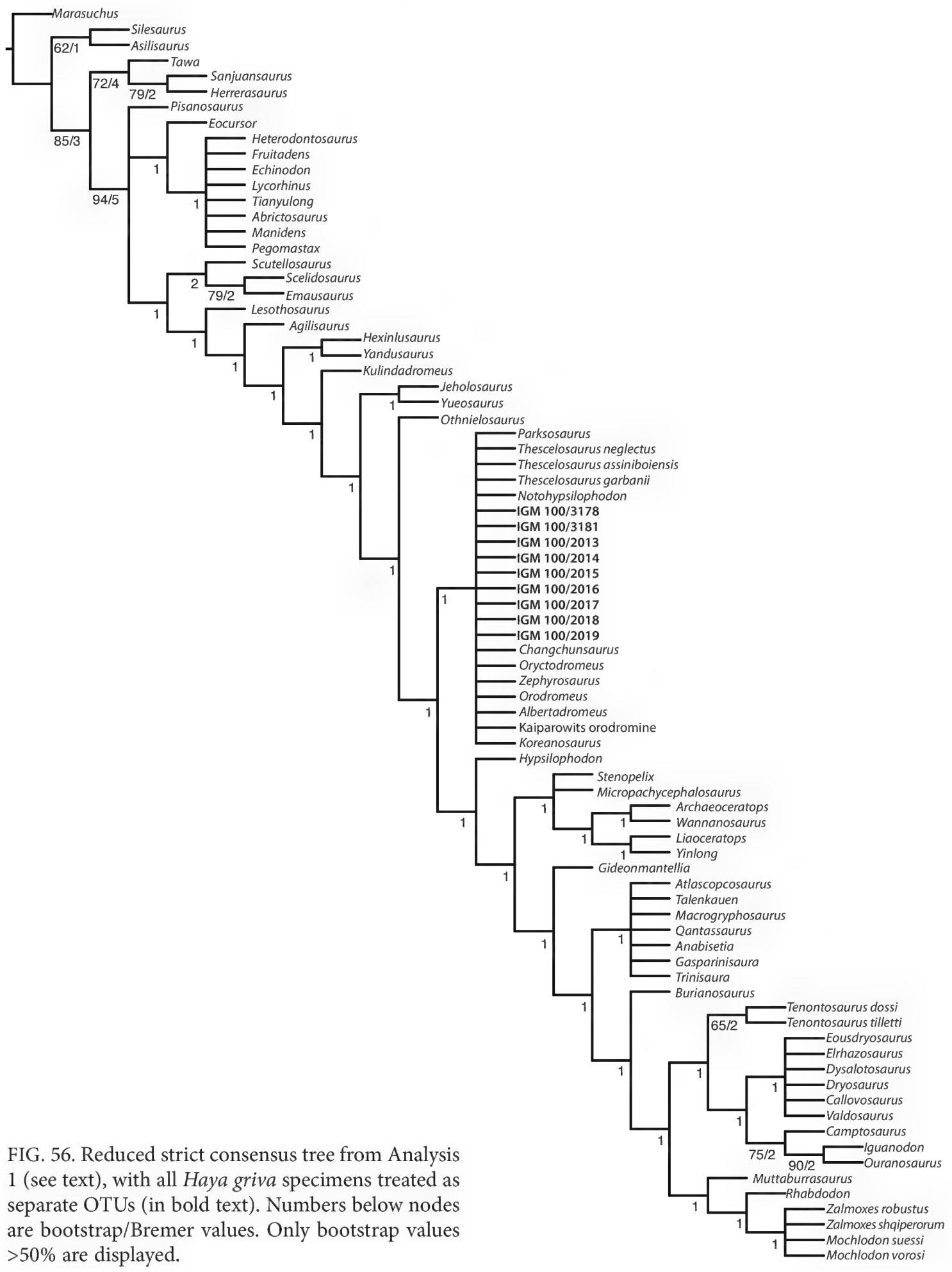




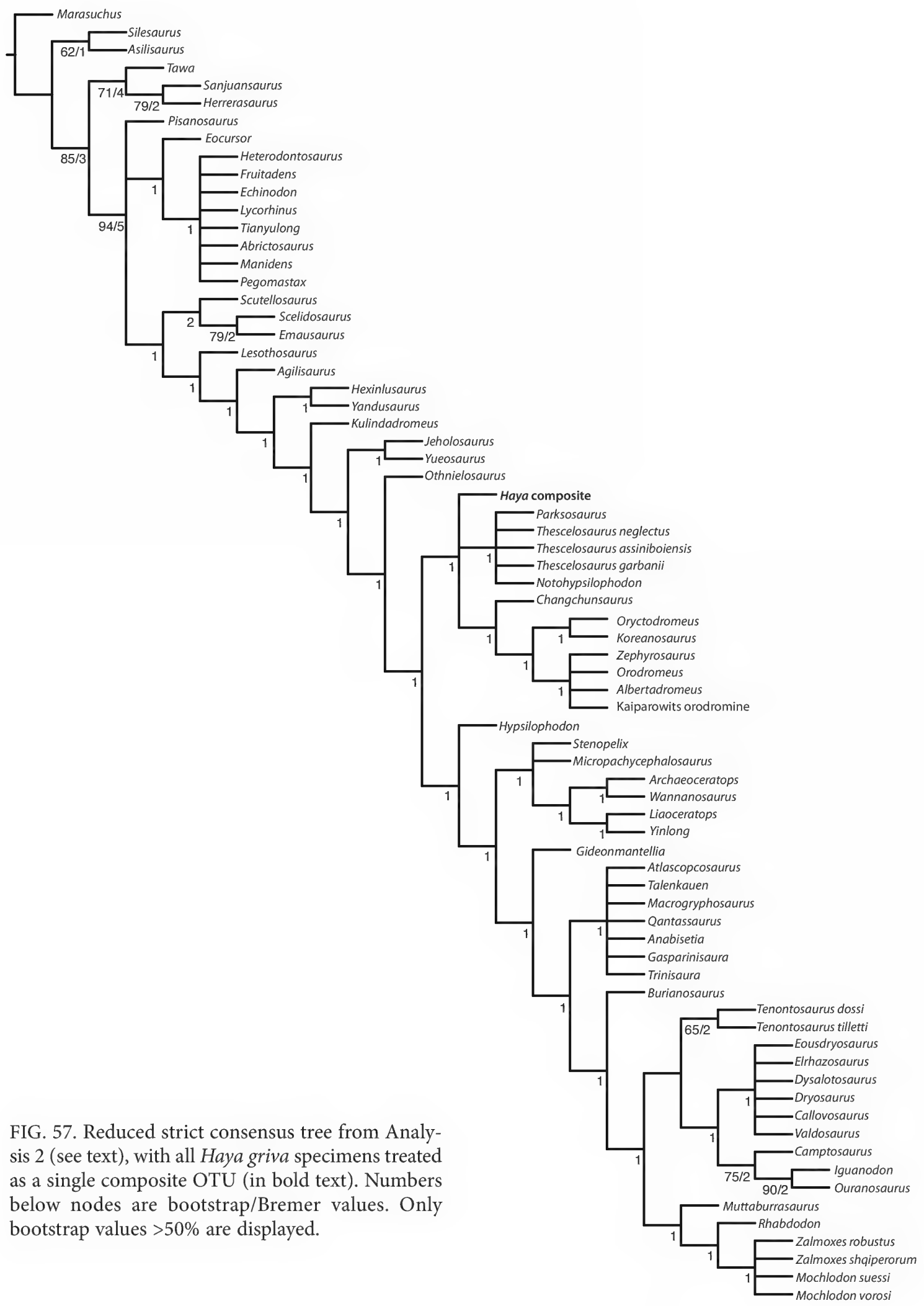




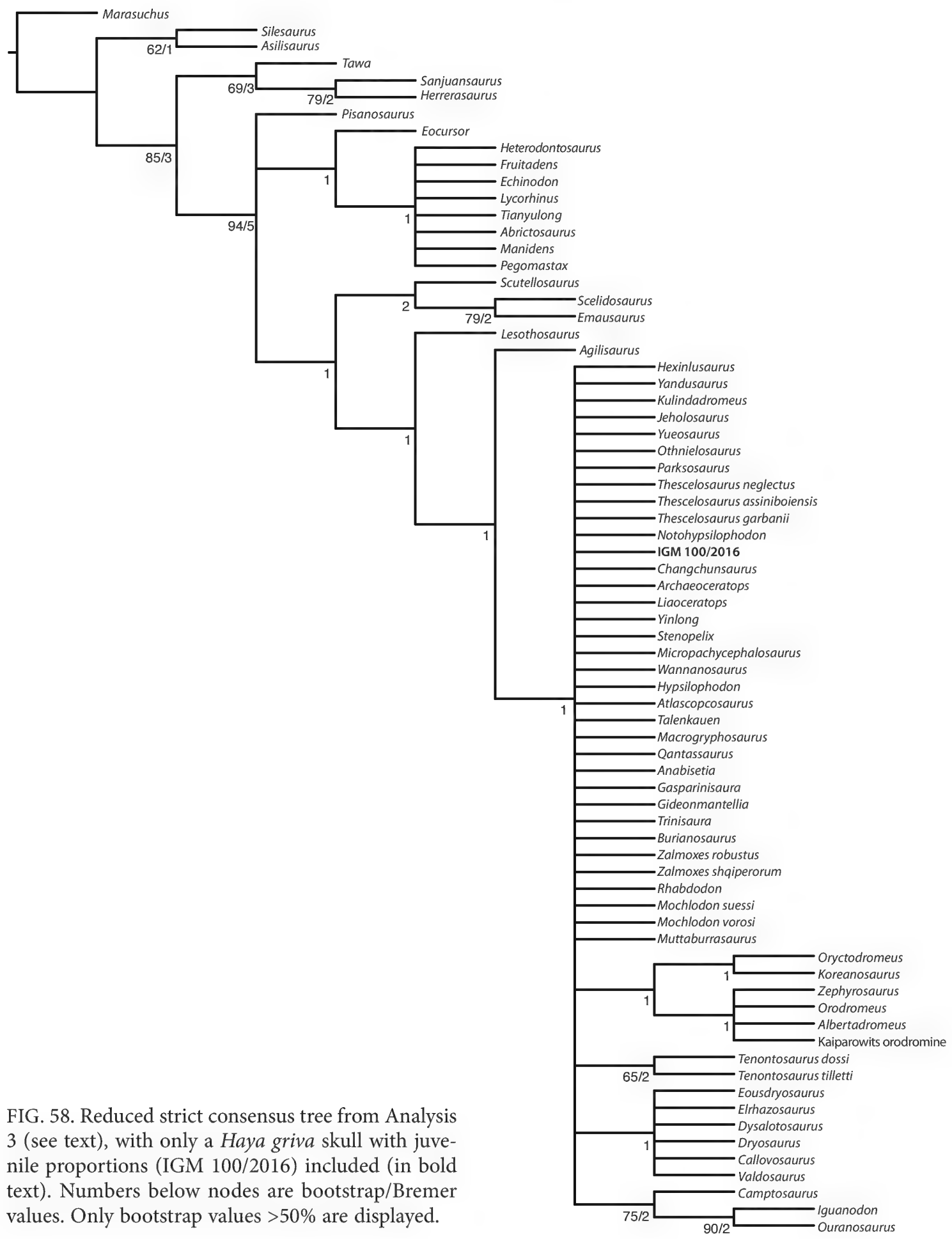


saurus does not appreciably improve these support values.

AnAlysis 3: This analysis was run with the same parameters as Analysis 1 above, with the exception that only IGM 100/2016, a Haya skull with juvenile morphology, was included. We recovered 100,000 trees of 897 steps (CI: 0.344, RI: 0.640 ), hitting the limit of tree storage we defined. After examining the strict consensus tree, Leallynasaura and Morrosaurus were then pruned a posteriori, as in analyses 1 and 2 .

Nearly all of Neornithischia collapses within a large polytomy on the strict consensus resulting from this analysis. Following pruning of Leallynasaura and Morrosaurus, the topology is slightly more resolved, though much of Neornithischia remains a polytomy. The position of Haya within Neornithischia is similarly unresolved (fig. 58). As for analyses 1 and 2, clades less inclusive than Ornithischia have very low Bremer support and bootstrap values, typically 1 and less than 50, respectively. Pruning Leallynasaura and Morrosaurus does not appreciably improve these support values.

AUtAPOMORPHIES OF Haya griva: In addition to the features listed in the differential diagnosis in the Systematic Paleontology section, we provide a list of features recovered as "unambiguous autapomorphies" for the composite Haya griva OTU in Analysis 2, using the apo[ command in TNT. Clearly these are not true unambiguous autapomorphies, because we parenthetically note instances in which some features are present in other analyzed taxa. This list is perhaps best thought of as a combination of character states that may add to the differential diagnosis of Haya griva provided in the Systematic Paleontology section of this work.

The combination of character states diagnosing Haya griva that emerged from the analysis is: (1) smooth anterodorsal surface of the premaxilla (char. 12: state 0), (2) quadratojugal anteroposteriorly short and dorsoventrally tall (43: 1) (also in Manidens and Rhabdodontomorpha), (3) ventral extent of the jugal wing of the quadrate positioned above the distal end of the quadrate (49: 1) (also in Oryctodromeus), (4) maxillary fenestra present (92:1) (also in Hypsilophodon and Kulindadromeus), (5) lingual surface of maxillary teeth flat (122: 2), (6) 10 or more ridges present on dentary teeth (138: 1) (also in Thescelosaurus and Atlascopcosaurus), (7) epipophyses on anterior cervical vertebra three present (145:0) (also in Lesothosaurus, Heterodontosaurus, Anabisetia, Talenkauen, and Macrogryphosaurus [Santa Luca, 1980; Sereno, 1991; Novas et al., 2004; Calvo et al., 2007; Rozadilla et al., 2019; 2020]), (8) length of sternal process of coracoid greater than $70 \%$ the width of the sternal process (162: 1) (also in Koreanosaurus), (9) shaft of the humerus straight (167:0) (also in the Yueosaurus + Jeholosaurus clade and Dryomorpha), (10) medial femoral condyle inflated and at least partially covering the intercondylar groove (224: 1) (also in some elasmarians and iguanodontians [Salgado et al., 1997; Bell et al., 2019]), (11) posterior intercondylar groove extends greater than 1/4 the length of the femur (225: 1) (also in Silesauridae), (12) cnemial crest of tibia straight (230: 0$)$.

The broader taxonomic distribution of character states recovered as autapomorphies of Haya griva in our analysis suggests that further reevaluation of character construction and scoring in the data matrix of Madzia et al. (2018) is needed for multiple taxa, but this is beyond the scope of the current study.

\section{DISCUSSION}

\section{ONTOGENETIC AND Other INTRASPECIFIC VARIATION}

Our histological analysis reveals that all examined Haya specimens fall within the size range of those with skeletally immature femora. What can be concluded about the ontogenetic status of specimens that do not preserve long bones for histological analysis? What morphological changes are evident in the Haya partial growth series? IGM 100/2016 (fig. 10) clearly exhibits 
typical juvenile amniote characters (e.g., comparatively larger eyes and shorter rostrum and dentary) compared to the other Haya skulls, and we can initially hypothesize that it is of a relatively younger ontogenetic stage. The rostrum and dentary of IGM 100/2016 are indeed proportionally shorter than those of the holotype (IGM 100/2017) and IGM 100/2019. However, the three specimens have nearly the same basicranial widths and orbit heights (especially if the crushed orbit of IGM 100/2017 is taken into account) (fig. 3, table 1). Three hypotheses may explain this apparent discrepancy:

(1) IGM $100 / 2016$ is at the same ontogenetic stage as the other two specimens and there is intraspecific variation in rostrum length at this stage;

(2) IGM $100 / 2016$ is a younger juvenile that achieved "mature" basicranial dimensions similar to those of the other two relatively more mature specimens before its rostrum elongated;

(3) IGM 100/2016 is either a juvenile or paedomorphic adult of a different taxon.

Hypothesis 3 is highly unlikely given that IGM 100/2016 lacks any autapomorphies other than those that plausibly relate to proportional changes through ontogeny. Likewise, there is no support for this idea from our specimen-level cladistic analysis, as IGM 100/2016 is recovered in a polytomy with all other Haya and thescelosaurids. Testing hypothesis 1 would require a larger sample size of Haya skulls with juvenile morphology, something that is not currently available. The rate of change in facial proportions and the amount of size variance within a given ontogenetic stage therefore remain unknown at this time. We must consider both hypotheses 1 and 2 plausible, though indicators of relative ontogenetic stage other than rostrum length described below lend support to hypothesis 2 .

Assessments of ontogenetic stage from morphology should not be made on just one or two characters alone (Hübner and Rauhut, 2010). Fortunately, there are other ontogenetic differences between IGM 100/2016 and the other Haya skulls that can help to determine its relative maturity. The posterior skull region of IGM $100 / 2016$ is dorsoventrally shorter than those of other specimens. The parietal and occipital region face posteroventrally in IGM 100/2016 (fig. 10), as in immature Dryosaurus and Dysalotosaurus (Carpenter, 1994; Hübner and Rauhut, 2010). They face directly posteriorly in more mature Dryosaurus and Dysalotosaurus and the other Haya specimens. Other similarities between juvenile Dyslaotosaurus and IGM 100/2016 are poorly defined supraoccipital and sagittal crests (Hübner and Rauhut, 2010). As in juvenile Dysalotosaurus, the exoccipital and opisthotic are not fused in IGM 100/2016, and there is probably a visible suture between the exoccipital and basioccipital in both taxa (Hübner and Rauhut, 2010), in contrast to Orodromeus, where they are fused even in small juveniles (Scheetz, 1999). Braincase variation in Orodromeus is not as straightforward, however. In that taxon, small specimens are observed to have closed opisthotic-basioccipital sutures, whereas they are unfused in larger specimens (Scheetz, 1999). Therefore, the absence of opisthotic-basioccipital fusion in IGM 100/2016 cannot be taken as evidence for skeletally immature status a priori. Jeholosaurus develops rugosities on its premaxilla and jugal during ontogeny (Barrett and Han, 2009). All Haya specimens lack any such rugosity, and this optimizes as an autapomorphy for Haya in our analysis. However, without Haya skulls of a more advanced ontogenetic stage, it is impossible to know whether Haya lacked rugosity throughout its life or whether it would have developed later in ontogeny. Likewise, the jugal and lacrimal do not contact each other in juveniles of Jeholosaurus and Dysalotosaurus, or any Haya, but they do contact in more mature Jeholosaurus and Dysalotosaurus (Barrett and Han, 2009; Hübner and Rauhut, 2010). Therefore, we cannot be certain whether this is a shared juvenile feature among Haya, Jeholosaurus, and Dysalotosaurus, or a true phylogenetic character difference between them. The orbital margin of the postorbital is slightly more rugose in IGM 100/2016 than in other Haya, which may simply 
TABLE 1

Measurements of selected Haya griva specimens (in mm).

\begin{tabular}{|c|c|c|c|c|c|c|c|c|c|c|}
\hline Element & IGM & IGM & IGM & IGM & IGM & IGM & IGM & IGM & IGM & IGM \\
\hline - & $100 / 2013$ & $100 / 2014$ & $100 / 2015$ & $100 / 2016$ & $100 / 2017$ & $100 / 2019$ & $100 / 3137$ & $100 / 3178$ & $100 / 3181$ & $100 / 3182$ \\
\hline Skull length ${ }^{1}$ & & & & & 80.3 & & & 83.8 & 86.5 & \\
\hline $\begin{array}{l}\text { Mandible } \\
\text { length }\end{array}$ & & 89.5 & & $53.12(\mathrm{i})$ & 80.9 & 75.9 (i) & & 85.5 & & \\
\hline Dentary length & & & & 42.7 & 50.7 & & & & & \\
\hline $\begin{array}{l}\text { Maxillary } \\
\text { toothrow length }\end{array}$ & & & & & 32.9 & & & 33.7 & & \\
\hline $\begin{array}{l}\text { Quadrate, dor- } \\
\text { soventral height }\end{array}$ & & & & & & 40.5 & & 36.6 & & \\
\hline $\begin{array}{l}\text { Orbit, antero- } \\
\text { posterior length }\end{array}$ & & & & 23.8 & 35.9 & 35.2 & & 34.0 & 36.0 & \\
\hline $\begin{array}{l}\text { Orbit, dorso- } \\
\text { ventral height }\end{array}$ & & & & 28.1 & 21.2 & 28.8 & & 28.4 & 35.3 & \\
\hline $\begin{array}{c}\text { Basicranial } \\
\text { width }^{2}\end{array}$ & & & & 38.9 & 34.1 (i) & 36.4 & & & & \\
\hline Cervical series & & & 150.7 & & & 145.4 & & $138.6(\mathrm{i})$ & & \\
\hline Dorsal series & & & 213.2 & & & 276.9 & & & & \\
\hline Sacral series & & & 113.2 & & & 130.5 & & & & \\
\hline Caudal series & & & 294 (i) & & & $63.2(\mathrm{i})$ & 337 (i) & & & \\
\hline Scapula & & & 90.9 & & & 90.0 & & 80.6 & & \\
\hline Coracoid & & & 52.5 & & & & & & & \\
\hline Sternum & & & 51.9 & & & & & & & \\
\hline Humerus & & & 86.5 & & & & & 87.4 & & \\
\hline Ulna & & & 42.1 (i) & & & & 42 (i) & & & \\
\hline Radius & & & 67.0 & & & & & & & \\
\hline $\mathrm{mcI}$ & & & 11.5 & & & & & & & \\
\hline mcII & & & 14.7 & & & & & & & \\
\hline mcIII & & & 13.2 & & & & & & & \\
\hline $\mathrm{mcIV}$ & & & 9.6 (i) & & & & & & & \\
\hline $\begin{array}{l}\text { Ilium, antero- } \\
\text { posterior length }\end{array}$ & & & 141.3 & & & 150.4 & & 113.3 & & \\
\hline $\begin{array}{l}\text { Ilium, dorso- } \\
\text { ventral height }{ }^{3}\end{array}$ & & & 39.0 & & & 43.7 & & 31.6 & & \\
\hline Pubis & & & 205.4 & & & & & & & \\
\hline Ischium & & & 134.1 & & & 76.0 & 124.5 (i) & & & \\
\hline Femur & 129.0 & & 128.9 & & & 147.9 (i) & $\begin{array}{l}137.41 \\
(\mathrm{i})^{4}\end{array}$ & 135.4 & & 141.5 \\
\hline Tibia & 183.9 & & 155.3 & & & & 147.3 & 121 (i) & & 159.8 \\
\hline Fibula & 166.9 & & & & & & & & & \\
\hline
\end{tabular}


TABLE 1 continued

\begin{tabular}{|c|c|c|c|c|c|c|c|c|c|c|}
\hline Element & $\begin{array}{c}\text { IGM } \\
100 / 2013\end{array}$ & $\begin{array}{c}\text { IGM } \\
100 / 2014\end{array}$ & $\begin{array}{c}\text { IGM } \\
100 / 2015\end{array}$ & $\begin{array}{c}\text { IGM } \\
100 / 2016\end{array}$ & $\begin{array}{c}\text { IGM } \\
100 / 2017\end{array}$ & $\begin{array}{c}\text { IGM } \\
100 / 2019\end{array}$ & $\begin{array}{c}\text { IGM } \\
100 / 3137\end{array}$ & $\begin{array}{c}\text { IGM } \\
100 / 3178\end{array}$ & $\begin{array}{c}\text { IGM } \\
100 / 3181\end{array}$ & $\begin{array}{c}\text { IGM } \\
100 / 3182\end{array}$ \\
\hline $\begin{array}{c}\text { Astragalus, } \\
\text { mediolateral } \\
\text { width }\end{array}$ & 38.3 & & & & & & & 25.3 & & \\
\hline $\mathrm{mtI}$ & 50.6 & & & & & & 43.2 & 26.9 & & \\
\hline mtII & 80.2 & & & & & & 62.5 & 52.6 & & \\
\hline mtIII & 89.9 & & & & & & & 61.3 & & $66.5(\mathrm{i})$ \\
\hline $\mathrm{mtIV}$ & 79.5 & & & & & & & 52.0 & & \\
\hline $\mathrm{mtV}$ & 23.0 & & & & & & & (i) & & \\
\hline pedal phalanx I-1 & 27.9 & & & & & & 22.2 & 18.4 & & \\
\hline pedal phalanx I-2 & 23.2 & & & & & & 15.0 & 15.8 & & \\
\hline pedal phalanx II-1 & 28.8 & & & & & & 21.6 & 20.1 & & \\
\hline pedal phalanx II-2 & 20.2 & & & & & & 14.7 & 15.4 & & \\
\hline pedal phalanx II-3 & 29.3 & & & & & & 21.3 & 20.3 & & \\
\hline $\begin{array}{c}\text { pedal phalanx } \\
\text { III-1 }\end{array}$ & 26.1 & & & & & & & 20.1 & & \\
\hline $\begin{array}{c}\text { pedal phalanx } \\
\text { III-2 }\end{array}$ & 18.1 & & & & & & & 14.7 & & \\
\hline $\begin{array}{c}\text { pedal phalanx } \\
\text { III-3 }\end{array}$ & 17.5 & & & & & & 13.9 & 12.7 & & \\
\hline $\begin{array}{c}\text { pedal phalanx } \\
\text { III-4 }\end{array}$ & 31.8 & & & & & & 18.8 & 23.0 & & \\
\hline $\begin{array}{c}\text { pedal phalanx } \\
\text { IV-1 }\end{array}$ & 16.5 & & & & & & & 13.3 & & \\
\hline $\begin{array}{c}\text { pedal phalanx } \\
\text { IV-2 }\end{array}$ & 15.5 & & & & & & & 11.4 & & \\
\hline $\begin{array}{l}\text { pedal phalanx } \\
\text { IV-3 }\end{array}$ & 11.1 & & & & & & & 9.8 & & \\
\hline $\begin{array}{l}\text { pedal phalanx } \\
\text { IV-4 }\end{array}$ & 10.0 & & & & & & & 8.8 & & \\
\hline $\begin{array}{l}\text { pedal phalanx } \\
\text { IV-5 }\end{array}$ & 25.1 & & & & & & & 14.1 & & \\
\hline
\end{tabular}

${ }^{1}$ From tip of premaxilla to posterior border of quadrate.

${ }^{2}$ Across paraoccipital processes.

${ }^{3}$ From ischial peduncle to dorsal border.

${ }^{4}$ Estimated from both right and left femora.

be an individually variable trait and not reflective of ontogeny.

The dentary is straight in IGM 100/2016, whereas it bows medially in IGM 100/2017. IGM $100 / 2016$ has the fewest dentary alveoli with 10-12, IGM 100/2014 and IGM 100/3178 have
13-14, and IGM 100/2017 has the most with 15-16. This increase in the number of teeth through ontogeny is typical of ornithischians and many squamates, whereas the change in tooth number is more variable in crocodylians and theropods (Brown et al., 2015). No change in the 
shape of the dentary teeth is evident between IGM 100/2016 and the other Haya specimens, contrasting with Dysalotosaurus, in which the dentary teeth widen through ontogeny, and Orodromeus, in which they become narrower. Makovicky et al. (2011) stated that the frontals become anteroposteriorly longer and mediolaterally narrower through ontogeny, opposite to the shortening/widening of the frontals with age in Dysalotosaurus (Hübner and Rauhut, 2010). However, our measurements of the frontals show that there is no major difference in frontal length:width ratio between IGM 100/2016 and IGM 100/2017. Caudal neurocentral sutures are mostly fused in Haya, whereas those of the dorsal and cervical series are not. This posterior to anterior sequence of neurocentral suture fusion agrees with the data for early diverging ornithischians summarized by Irmis (2007). The same pattern was also described in Jeholosaurus (Han et al., 2012).

Together, the features above suggest that IGM $100 / 2016$ is a juvenile skull that attained a basicranial width nearly equal to comparatively more mature Haya specimens before its craniofacial proportions and sutures attained a more mature condition. This potentially indicates size variation among Haya at a given ontogenetic stage, though more Haya skulls with juvenile proportions are needed to test the degree to which size and relative age correlate. Broad-scale proportional changes of the cranium and the pattern of neurocentral suture fusion are shared between Haya and other early-diverging neornithischian taxa for which growth series are known, but Haya differs in some specific features of its ontogeny, such as a lack of apparent change in dentary tooth morphology. Thus, although the ontogeny of Haya appears relatively conservative, some differences in ontogenetic trajectory may prove to be phylogenetically distinctive.

\section{Phylogenetic Position of Haya}

Haya appears as a thescelosaurid or the closest sister taxon to Thescelosauridae in all derivatives of the Boyd (2015) matrix (figs. 56-58). Haya was originally recovered as a thescelosaurine by Boyd (2015), then as an orodromine by Madzia et al. (2018). Our analysis cannot distinguish between these two alternatives, or one in which Haya is outside either clade as the sister taxon to Thescelosauridae as defined by Madzia et al. (2018). As in previous work stemming from the Boyd (2015) analysis, Haya is not part of an exclusively Asian clade of early-diverging neornithischians in our analysis, instead belonging to or the nearest relative of the Thescelosauridae, which, apart from Haya and Koreanosaurus, has a North American distribution.

The results of the specimen level analaysis (fig. 56) are consistent with all the specimens belonging to a single taxon, though the specimens do not group together in a clade to the exclusion of all other ornithischians. This is likely due to slight individual and ontogenetic variations. None of the Haya specimens were recovered as an exclusive sister OTU to a different taxon or as members of a phylogenetically distant clade, either of which might be expected if multiple taxa were present in our sample.

The phylogenetic position of Haya does not seem to be sensitive to the effects of ontogeny. IGM 100/2016 is placed near the other specimens in the specimen-level analysis (fig. 56), and its position relative to other neornithischians is very poorly resolved when it is the only Haya included (fig. 58). This may be because IGM 100/2016 lacks postcrania and therefore is missing data that could help place it more precisely. Nevertheless, there is no evidence to suggest that immature cranial morphology would cause it to appear in an earlier-diverging position.

Haya is recovered as an ornithopod in Han et al's (2018) analysis, which also finds Thescelosauridae to be paraphyletic. The position of Haya is not well resolved in that analysis, as it is part of a large polytomy on the strict consensus tree, and its placement is not supported by bootstrap values $>50 \%$ or Bremer values $>1$ 
on the reduced consensus tree (Han et al., 2018). We will seek to integrate our new morphological information on Haya into the Han et al. (2018) matrix in the future.

Due to the low levels of clade support in our analysis and previous iterations of the Boyd (2015) matrix, as well as conflicting hypotheses of neornithischian relationships compared to the analysis of Han et al. (2018), future work should build on detailed descriptions of key taxa, such as that presented here for Haya, to identify novel characters to help resolve the interrelationships of ornithischians. Future workers should strive to further integrate the character sets of Boyd (2015) and Han et al. (2018) as well. We are confident that this work lays the groundwork for further study of Haya griva and its relatives that may lead to a clearer picture of ornithischian phylogeny.

\section{SUMMARY}

Haya griva is the only early-diverging neornithischian taxon from Mongolia, and is known from many excellently preserved specimens that provide a wealth of information about its skeletal anatomy, growth, and phylogenetic relationships. It now is one of the most completely known of the "hypsilophodontid" dinosaurs. Furthermore, based on the current temporal distribution of finds, it serves as an important biostratigraphic marker for correlating older strata in the eastern Gobi with younger strata in the western Gobi to provide a more complete terrestrial Cretaceous stratigraphic section in central Asia. Age determination of Haya specimens spanning the currently known size range clarifies that, as is perhaps true for most small dinosaurs, most individuals rarely lived to reach skeletal maturity. We hope that this description, with novel anatomical information from observations and CT scanning, will enhance an increasing body of knowledge about primitive ornithischian taxa that will provide further clarity about their fluctuating interrelationships.

\section{ACKNOWLEDGMENTS}

We thank the Mongolian Academy of Sciences and the 2002-2007, 2015, and 2016 field crews of the Mongolian Academy of SciencesAmerican Museum of Natural History Gobi Expeditions (MAE) for excavating the specimens. We especially thank our late friend and colleague Tamir Nasankhuu and dedicate this work to him in memory of his extraordinary contributions to Mongolian paleontology. Specimens were prepared by Amy Davidson, Lisa Herzog, Verne Lee, Akiko Shinya, and Debbie Wagner. Ana Balcarcel, Amy Davidson, and Verne Lee provided histology techniques advice. We thank Mick Ellison for his excellent photographs that form the solid foundation for the rest of this monograph. Nicole Wong skillfully rendered the line drawings in figures 3 and 4 . Collections access and assistance were provided by Amy Atwater, John Scannella (MOR), Dan Brinkman, Chris Norris, Bhart-Anjan Bhullar, Jacques Gauthier (YPM), Kevin Seymour, David Evans (ROM), and Carl Mehling (AMNH). Mike D'Emic provided access to the Zeiss Axio Imager 72 Automated Microscope Imaging System. Bernardo Santos provided a TNT script for converting TNT trees to Newick format. Eugenia Gold, John Flynn, Ashley Heers, Melanie Hopkins, Pete Makovicky, Jin Meng, Rebecca Pian, John Scannella, Jade Simon, Aki Watanabe, and Holly Woodward Ballard provided helpful discussion. We thank John Flynn for his comments on a draft of the manuscript. We thank the editor, Jin Meng, and reviewers Phil Bell and Sebastián Rozadilla for their thorough, constructive comments on the manuscript. D.E.B. is supported by the Oklahoma State University Center for Health Sciences, a Richard Gilder Graduate School fellowship, the AMNH Division of Paleontology, and National Science Foundation Doctoral Dissertation Improvement Grant \#DEB-1601315. The Macaulay Family Endowment and AMNH Division of Paleontology provide funding to M.A.N. TNT is made available by the Willi Hennig Society. Translations of He and Cai (1984) 
and Peng (1992) by Will Downs were obtained courtesy of the Polyglot Paleontologist website (http://www.paleoglot.org).

\section{REFERENCES}

Andrzejewski, K.A., D.A. Winkler, and L.L. Jacobs. 2019. A new basal ornithopod (Dinosauria: Ornithischia) from the Early Cretaceous of Texas. PLoS ONE 14: 0207935.

Baron, M.G., D.B. Norman, and P.M. Barrett. 2017. Postcranial anatomy of Lesothosaurus diagnosticus (Dinosauria: Ornithischia) from the Lower Jurassic of southern Africa: implications for basal ornithischian taxonomy and systematics. Zoological Journal of the Linnean Society 179:125-168.

Barrett, P.M., and F.-L. Han. 2009. Cranial anatomy of Jeholosaurus shangyuanensis (Dinosauria: Ornithischia) from the Early Cretaceous of China. Zootaxa 2072: 31-55.

Barrett, P.M., R.J. Butler, and F. Knoll. 2005. Smallbodied ornithischian dinosaurs from the Middle Jurassic of Sichuan, China. Journal of Vertebrate Paleontology 25: 823-834.

Barrett, P.M., et al. 2014. A palaeoequatorial ornithischian and new constraints on early dinosaur diversification. Proceedings of the Royal Society of London B, Biological Sciences 281: 20141147.

Barta, D.E., S.J. Nesbitt, and M.A. Norell. 2018. The evolution of the manus of early theropod dinosaurs is characterized by high inter- and intraspecific variation. Journal of Anatomy 232: 80-104.

Bell, P. R., T. Brougham, M.C. Herne, T. Frauenfelder, and E.T. Smith. 2019. Fostoria dhimbangunmal, gen. et sp. nov., a new iguanodontian (Dinosauria, Ornithopoda) from the mid-Cretaceous of Lightning Ridge, New South Wales, Australia. Journal of Vertebrate Paleontology: e1564757.

Boyd, C.A. 2014. The cranial anatomy of the neornithischian dinosaur Thescelosaurus neglectus. PeerJ 2: e669.

Boyd, C.A. 2015. The systematic relationships and biogeographic history of ornithischian dinosaurs. PeerJ 3: e1523.

Boyd, C.A., C.M. Brown, R.D. Scheetz, and J.A. Clarke. 2009. Taxonomic revision of the basal neornithischian taxa Thescelosaurus and Bugenasaura. Journal of Vertebrate Paleontology 29: 758-770.

Brown, B., and E.M. Schlaikjer. 1940. A new element in the ceratopsian jaw with additional notes on the mandible. American Museum Novitates 1092: 1-13.
Brown, C.M., C.A. Boyd, and A.P. Russell. 2011. A new basal ornithopod dinosaur (Frenchman Formation, Saskatchewan, Canada), and implications for late Maastrichtian ornithischian diversity in North America. Zoological Journal of the Linnean Society 163: 1157-1198.

Brown, C.M., D.C. Evans, M.J. Ryan, and A.P. Russell. 2013. New data on the diversity and abundance of small-bodied ornithopods (Dinosauria, Ornithischia) from the Belly River Group (Campanian) of Alberta. Journal of Vertebrate Paleontology 33: 495-520.

Brown, C.M., et al. 2015. Tooth counts through growth in diapsid reptiles: implications for interpreting individual and size-related variation in the fossil record. Journal of Anatomy 226: 322-333.

Bruner, H.L. 1907. On the cephalic veins and sinuses of reptiles, with description of a mechanism for raising the venous blood-pressure in the head. Developmental Dynamics 7: 1-117.

Brusatte, S.L., et al. 2013. An infant ornithopod dinosaur tibia from the Late Cretaceous of Sebeş, Romania. Terra Sebus. Acta Musei Sabesiensis 5: 627-644.

Butler, R.J. 2010. The anatomy of the basal ornithischian dinosaur Eocursor parvus from the lower Elliot Formation (Late Triassic) of South Africa. Zoological Journal of the Linnean Society 160: 648-684.

Butler, R.J., and P.M. Galton. 2008. The 'dermal armour' of the ornithopod dinosaur Hypsilophodon from the Wealden (Early Cretaceous: Barremian) of the Isle of Wight: a reappraisal. Cretaceous Research 29: 636-642.

Butler, R.J., J. Liyong, C. Jun, and P. Godefroit. 2011. The postcranial osteology and phylogenetic position of the small ornithischian dinosaur Changchunsaurus parvus from the Quantou Formation (Cretaceous: Aptian-Cenomanian) of Jilin Province, north-eastern China. Palaeontology 54: 667-683.

Butler, R.J., L.B. Porro, P.M. Galton, and L.M. Chiappe. 2012. Anatomy and cranial functional morphology of the small-bodied dinosaur Fruitadens haagarorum from the Upper Jurassic of the USA. PLoS ONE 7: e31556.

Bybee, P.J., A.H. Lee, and E.-T. Lamm. 2006. Sizing the Jurassic theropod dinosaur Allosaurus: assessing growth strategy and evolution of ontogenetic scaling of limbs. Journal of Morphology 267: 347-359.

Cabreira, S.F., et al. 2011. New stem-sauropodomorph (Dinosauria, Saurischia) from the Triassic of Brazil. Naturwissenschaften 98: 1035-1040.

Calvo, J.O., J.D. Porfiri, and F.E. Novas. 2007. Discovery of a new ornithopod dinosaur from the Portezuelo for- 
mation (Upper Cretaceous), Neuquen, Patagonia, Argentina. Arquivos do Museu Nacional 65: 471-483. Campione, N.E. 2013. MASSTIMATE: Body mass estimation equations for vertebrates. Online resource (https://cran.r-project.org/web/packages/MASSTIMATE/index.html).

Campione, N.E., K.S. Brink, E.A. Freedman, C.T. McGarrity, and D.C. Evans. 2013. 'Glishades ericksoni', an indeterminate juvenile hadrosaurid from the Two Medicine Formation of Montana: implications for hadrosauroid diversity in the latest Cretaceous (Campanian-Maastrichtian) of western North America. Palaeobiodiversity and Palaeoenvironments 93: 65-75.

Campione, N.E., D.C. Evans, C.M. Brown, and M.T. Carrano. 2014. Body mass estimation in non-avian bipeds using a theoretical conversion to quadruped stylopodial proportions. Methods in Ecology and Evolution 5: 913-923.

Carpenter, K. 1994. Baby Dryosaurus from the Upper Jurassic Morrison Formation of Dinosaur National Monument. In K. Carpenter, K. Hirsch, and J. Horner (editors), Dinosaur eggs and babies: 288297. Cambridge: Cambridge University Press.

Carpenter, K., and Y. Wilson. 2008. A new species of Camptosaurus (Ornithopoda: Dinosauria) from the Morrison Formation (Upper Jurassic) of Dinosaur National Monument, Utah, and a biomechanical analysis of its forelimb. Annals of Carnegie Museum 76: 227-263.

Castanet, J. 1986. La squelettochronologie chez les reptiles. III: application. Annales des Sciences Naturelles. Zoologie et Biologie Animale 8: 157-172.

Castanet, J., A. Grandin, A. Abourachid, and A. de Ricqlès. 1996. Expression de la dynamique de croissance dans la structure de los périostique chez Anas platyrhyncos. Comptes Rendus de l'Académie des Sciences (série 3) Sciences de la Vie 319: 301-308.

Castanet, J., K. Curry Rogers, J. Cubo, and J. JacquesBoisard. 2000. Periosteal bone growth rates in extant ratites (ostriche and emu). Implications for assessing growth in dinosaurs. Comptes Rendus de l'Académie des Sciences (série 3) Sciences de La Vie 323: 543-550.

Castanet, J., et al. 2004. Lines of arrested growth in bone and age estimation in a small primate: Microcebus murinus. Journal of Zoology 263: 31-39.

Cerda, I.A., and A. Chinsamy. 2012. Biological implications of the bone microstructure of the Late Cretaceous ornithopod dinosaur Gasparinisaura cincosaltensis. Journal of Vertebrate Paleontology 32: 355-368.
Chinsamy, A. 1995. Ontogenetic changes in the bone histology of the Late Jurassic ornithopod Dryosaurus lettowvorbecki. Journal of Vertebrate Paleontology 15: 96-104.

Chinsamy, A., T. Rich, and P. Vickers-Rich. 1998. Polar dinosaur bone histology. Journal of Vertebrate Paleontology 18: 385-390.

Claessens, L.P.A.M. 2004. Dinosaur gastralia; origin, morphology, and function. Journal of Vertebrate Paleontology 24: 89-106.

Colbert, E.H. 1945. The hyoid bones in Protoceratops and in Psittacosaurus. American Museum Novitates 1301: $1-10$.

Colbert, E.H., and J.H. Ostrom. 1958. Dinosaur stapes. American Museum Novitates 1900: 1-20.

Cooper, L.N., A.H. Lee, M.L. Taper, and J.R. Horner. 2008. Relative growth rates of predator and prey dinosaurs reflect effects of predation. Proceedings of the Royal Society B, Biological Sciences 275: 2609-2615.

Coria, R.A., and L. Salgado. 1996. A basal iguanodontian (Ornithischia: Ornithopoda) from the Late Cretaceous of South America. Journal of Vertebrate Paleontology 16: 445-457.

Coria, R.A., A.V. Cambiaso, and L. Salgado. 2007. New records of basal ornithopod dinosaurs in the Cretaceous of North Patagonia. Ameghiniana 44: 473-477.

Currie, P.J. 2003. Cranial anatomy of tyrannosaurid dinosaurs from the Late Cretaceous of Alberta, Canada. Acta Palaeontologica Polonica 48: 191-226.

Curtin, A.J., A.A. Macdowell, E.G. Schaible, and V.L. Roth. 2012. Noninvasive histological comparison of bone growth patterns among fossil and extant neonatal elephantids using synchrotron radiation X-ray microtomography. Journal of Vertebrate Paleontology 32: 939-955.

Eberth, D.A., et al. 2009. Assignment of Yamaceratops dorngobiensis and associated redbeds at Shine Us Khudag (Eastern Gobi, Dorngobi Province, Mongolia) to the redescribed Javkhlant Formation (Upper Cretaceous). Journal of Vertebrate Paleontology 29: 295-302.

Edmund, A.G. 1957. On the special foramina in the jaws of many ornithischian dinosaurs. Contributions of the Royal Ontario Museum Division of Zoology and Palaeontology 48: 1-14.

Edmund, A.G. 1960. Tooth replacement phenomena in the lower vertebrates. Contributions, Life Sciences Division, Royal Ontario Museum 52: 1-190.

Erickson, G.M. 2005. Assessing dinosaur growth patterns: a microscopic revolution. Trends in Ecology and Evolution 20: 677-684. 
Erickson, G.M. 2014. On dinosaur growth. Annual Review of Earth and Planetary Sciences 42: 675697.

Erickson, G.M., and T.A. Tumanova. 2000. Growth curve of Psittacosaurus mongoliensis Osborn (Ceratopsia: Psittacosauridae) inferred from long bone histology. Zoological Journal of the Linnean Society 130: 551-566.

Erickson, G.M., P.J. Makovicky, B.D. Inouye, C.-F. Zhou, and K.-Q. Gao. 2009. A life table for Psittacosaurus lujiatunensis: initial insights into ornithischian dinosaur population biology. Anatomical Record 292: 1514-1521.

Evans, S.E. 2008. The skull of lizards and tuatara. Biology of the Reptilia 20: 1-347.

Fanti, F., P.J. Currie, and D. Badamgarav. 2012. New specimens of Nemegtomaia from the Baruungoyot and Nemegt Formations (Late Cretaceous) of Mongolia. PLoS ONE 7: e31330.

Fearon, J.L., and D.J. Varricchio. 2015. Morphometric analysis of the forelimb and pectoral girdle of the Cretaceous ornithopod dinosaur Oryctodromeus cubicularis and implications for digging. Journal of Vertebrate Paleontology 35: e936555.

Fearon, J.L., and D.J. Varricchio. 2016. Reconstruction of the forelimb musculature of the cretaceous ornithopod dinosaur Oryctodromeus cubicularis: implications for digging. Journal of Vertebrate Paleontology 36: e1078341.

Forster, C.A. 1990. The postcranial skeleton of the ornithopod dinosaur Tenontosaurus tilletti. Journal of Vertebrate Paleontology 10: 273-294.

Francillon-Vieillot, H., et al. 1990. Microstructure and mineralization of vertebrate skeletal tissues. In J.G. Carter (editor), Skeletal biomineralization: patterns, processes, and evolutionary trends, vol. 1: 471-530. New York: Van Nostrand Reinhold.

Fürbringer, M. 1922. Das Zungenbein der Wirbeltiere insbesondere der Reptilien und Vögel. Nachgelassene Untersuchungen über systematische Phylogenie mit besonderer Berücksichtigung der Wurzel der Säugetiere. Berlin: Walter de Gruyter \& Co.

Galton, P.M. 1973. Redescription of the skull and mandible of Parksosaurus from the Late Cretaceous with comments of the family Hypsilophodontidae (Ornithischia). Contributions, Life Sciences Division, Royal Ontario Museum 89: 1-21.

Galton, P.M. 1974a. The ornithischian dinosaur Hypsilophodon from the Wealden of the Isle of Wight. Bulletin of the British Museum (Natural History) 25: $1-152$.
Galton, P.M. 1974b. Notes on Thescelosaurus, a conservative ornithopod dinosaur from the Upper Cretaceous of North America, with comments on ornithopod classification. Journal of Paleontology 48: 1048-1067.

Galton, P.M. 1981. Dryosaurus, a hypsilophodontid dinosaur from the Upper Jurassic of North America and Africa postcranial skeleton. Paläontologische Zeitschrift 55: 271-312.

Galton, P.M. 1983. The cranial anatomy of Dryosaurus, a hypsilophodontid dinosaur from the Upper Jurassic of North America and East Africa, with a review of hypsilophodontids from the Upper Jurassic of North America. Geologica et Palaeontologica 17: 207-243.

Galton, P.M. 1989. Crania and endocranial casts from ornithopod dinosaurs of the families Dryosauridae and Hypsilophodontidae (Reptilia: Ornithischia). Geologica et Palaeontologica 23: 217-239.

Galton, P.M. 2007. Teeth of ornithischian dinosaurs (mostly Ornithopoda) from the Morrison Formation (Upper Jurassic) of Western United States. In K. Carpenter (editor), Horns and beaks: ceratopsian and ornithopod dinosaurs: 17-47. Bloomington: Indiana University Press.

Galton, P.M. 2014. Notes on the postcranial anatomy of the heterodontosaurid dinosaur Heterodontosaurus tucki, a basal ornithischian from the Lower Jurassic of South Africa. Revue de Paléobiologie, Genève 33: 97-141.

Galton, P.M., and J.A. Jensen. 1973. Skeleton of a hypsilophodontid dinosaur (Nanosaurus (?) rex) from the Upper Jurassic of Utah. Brigham Young University Geology Studies 20: 137-157.

Galton, P.M., and P. Upchurch. 2004. Stegosauria. In D.B. Weishampel, P. Dodson, and H. Osmólska (editors), The Dinosauria: 343-362. Berkeley: University of California Press.

Gilmore, C.W. 1909. Osteology of the Jurassic reptile Camptosaurus: with a revision of the species of the genus, and description of two new species. Proceedings of the United States National Museum 36: 197-332.

Gilmore, C.W. 1915. Osteology of Thescelosaurus, an orthopodous dinosaur from the Lance Formation of Wyoming. Proceedings of the United States National Museum 49: 591-616.

Gilmore, C.W. 1925. Osteology of ornithopodous dinosaurs from Dinosaur National Monument, Utah. Part I. On a skeleton of Camptosaurus medius Marsh. Part II. On a skeleton of Dryosaurus altus Marsh. Part III. 
On a skeleton of Laosaurus gracilis Marsh. Memoirs of the Carnegie Museum 10: 385-410.

Godefroit, P., et al. 2014. A Jurassic ornithischian dinosaur from Siberia with both feathers and scales. Science 345: 451-455.

Goloboff, P.A., and S.A. Catalano. 2016. TNT version 1.5 , including a full implementation of phylogenetic morphometrics. Cladistics 32: 221-238.

Goloboff, P.A., J.S. Farris, and K.C. Nixon. 2008. TNT, a free program for phylogenetic analysis. Cladistics 24: 774-786.

Gower, D.J. 1999. The cranial and mandibular osteology of a new rauisuchian archosaur from the Middle Triassic of Southern Germany. Stuttgarter Beitrage zur Naturkunde, Serie B 280: 1-49.

Griffin, C.T., C.M. Stefanic, W.G. Parker, A. Hungerbühler, and M.R. Stocker. 2017. Sacral anatomy of the phytosaur Smilosuchus adamanensis, with implications for pelvic girdle evolution among Archosauriformes. Journal of Anatomy 231: 886-905.

Han, F., C.A. Forster, X. Xu, and J.M. Clark. 2018. Postcranial anatomy of Yinlong downsi (Dinosauria: Ceratopsia) from the Upper Jurassic Shishugou Formation of China and the phylogeny of basal ornithischians. Journal of Systematic Palaeontology 16: 1159-1187.

Han, F.-L., P.M. Barrett, R.J. Butler, and X. Xu. 2012. Postcranial anatomy of Jeholosaurus shangyuanensis (Dinosauria, Ornithischia) from the Lower Cretaceous Yixian Formation of China. Journal of Vertebrate Paleontology 32: 1370-1395.

Han, F., Q. Zhao, J. Stiegler, and X. Xu. 2020. Bone histology of the non-iguanodontian ornithopod Jeholosaurus shangyuanensis and its implications for dinosaur skeletochronology and development. Journal of Vertebrate Paleontology e1768538.

He, X.L., and K. Cai. 1984. The Middle Jurassic dinosaurian fauna from Dashanpu, Zigong, Sichuan. Vol. 1, The ornithopod dinosaurs. Chengdu: Sichuan Scientific and Technological Publishing House 71: 1-66.

Herne, M.C., et al. 2018. A new small-bodied ornithopod (Dinosauria, Ornithischia) from a deep, highenergy Early Cretaceous river of the Australian-Antarctic rift system. PeerJ 5: e4113.

Hill, R.V., M.D. D'Emic, G.S. Bever, and M.A. Norell. 2015. A complex hyobranchial apparatus in a Cretaceous dinosaur and the antiquity of avian paraglossalia. Zoological Journal of the Linnean Society 175: 892-909.

Holliday, C.M. 2009. New insights into dinosaur jaw muscle anatomy. Anatomical Record 292: 1246-1265.
Holmes, R., and C. Organ. 2007. An ossified tendon trellis in Chasmosaurus (Ornithischia: Ceratopsidae). Journal of Paleontology 81: 411-414.

Hopson, J.A. 1980. Tooth function and replacement in early Mesozoic ornithischian dinosaurs: implications for aestivation. Lethaia 13: 93-105.

Horner, J.R., and K. Padian. 2004. Age and growth dynamics of Tyrannosaurus rex. Proceedings of the Royal Society of London B, Biological Sciences 271: 1875-1880.

Horner, J.R., A. De Ricqlès, K. Padian, and R.D. Scheetz. 2009. Comparative long bone histology and growth of the "hypsilophodontid" dinosaurs Orodromeus makelai, Dryosaurus altus, and Tenontosaurus tillettii (Ornithischia: Euornithopoda). Journal of Vertebrate Paleontology 29: 734-747.

Hübner, T.R. 2012. Bone histology in Dysalotosaurus lettowvorbecki (Ornithischia: Iguanodontia) - variation, growth, and implications. PLoS ONE 7: e29958.

Hübner, T.R., and O.W.M. Rauhut. 2010. A juvenile skull of Dysalotosaurus lettowvorbecki (Ornithischia: Iguanodontia), and implications for cranial ontogeny, phylogeny, and taxonomy in ornithopod dinosaurs. Zoological Journal of the Linnean Society 160: 366-396.

Huh, M., D.-G. Lee, J.-K. Kim, J.-D. Lim, and P. Godefroit. 2011. A new basal ornithopod dinosaur from the Upper Cretaceous of South Korea. Neues Jahrbuch für Geologie und Paläontologie-Abhandlungen 259: 1-24.

Huxley, T.H. 1870. On Hypsilophodon foxii, a new dinosaurian from the Wealden of the Isle of Wight. Quarterly Journal of the Geological Society 26: 3-12.

Irmis, R.B. 2007. Axial skeleton ontogeny in the Parasuchia (Archosauria: Pseudosuchia) and its implications for ontogenetic determination in archosaurs. Journal of Vertebrate Paleontology 27: 350-361.

Janensch, W. 1936. Über bahnen von Hirnvenen bei Saurischiern und Ornithischiern, sowie einigen anderen Fossilen und rezenten Reptilien. Paläontologische Zeitschrift 18: 181-198.

Jin, L., J. Chen, S. Zan, R.J. Butler, and P. Godefroit. 2010. Cranial anatomy of the small ornithischian dinosaur Changchunsaurus parvus from the Quantou Formation (Cretaceous: Aptian-Cenomanian) of Jilin Province, northeastern China. Journal of Vertebrate Paleontology 30: 196-214.

Kammerer, C.F. 2011. Systematics of the Anteosauria (Therapsida: Dinocephalia). Journal of Systematic Palaeontology 9: 261-304. 
Kilbourne, B.M., and P.J. Makovicky. 2010. Limb bone allometry during postnatal ontogeny in non-avian dinosaurs: postnatal allometry of dinosaur limb bones. Journal of Anatomy 217: 135-152.

Kitchener, J.L., N.E. Campione, E.T. Smith, and P.R. Bell. 2019. High-latitude neonate and perinate ornithopods from the mid-Cretaceous of southeastern Australia. Scientific Reports 9: 19600.

Knoll, F. 2002a. New skull of Lesothosaurus (Dinosauria: Ornithischia) from the Upper Elliot Formation (Lower Jurassic) of southern Africa. Geobios 35: 595-603.

Knoll, F. 2002b. Nearly complete skull of Lesothosaurus (Dinosauria: Ornithischia) from the Upper Elliot Formation (Lower Jurassic: Hettangian) of Lesotho. Journal of Vertebrate Paleontology 22: 238-243.

Knoll, F. 2008. Buccal soft anatomy in Lesothosaurus (Dinosauria: Ornithischia). Neues Jahrbuch für Geologie und Paläontologie-Abhandlungen 248: 355-364.

Langer, M.C., and M.J. Benton. 2006. Early dinosaurs: a phylogenetic study. Journal of Systematic Palaeontology 4: 309-358.

LeBlanc, A.R.H., R.R. Reisz, D.C. Evans, and A.M. Bailleul. 2016. Ontogeny reveals function and evolution of the hadrosaurid dinosaur dental battery. BMC Evolutionary Biology 16: 152.

LeBlanc, A.R.H., K.S. Brink, T.M. Cullen, and R.R. Reisz. 2017. Evolutionary implications of tooth attachment versus tooth implantation: a case study using dinosaur, crocodilian, and mammal teeth. Journal of Vertebrate Paleontology 37: e1354006.

Madzia, D., C.A. Boyd, and M. Mazuch. 2018. A basal ornithopod dinosaur from the Cenomanian of the Czech Republic. Journal of Systematic Palaeontology 16: 967-979.

Maidment, S.C.R., and P.M. Barrett. 2011. The locomotor musculature of basal ornithischian dinosaurs. Journal of Vertebrate Paleontology 31: 1265-1291.

Maidment, S.C.R., and L.B. Porro. 2010. Homology of the palpebral and origin of supraorbital ossifications in ornithischian dinosaurs. Lethaia 43: 95-111.

Makovicky, P.J., B.M. Kilbourne, R.W. Sadleir, and M.A. Norell. 2011. A new basal ornithopod (Dinosauria, Ornithischia) from the Late Cretaceous of Mongolia. Journal of Vertebrate Paleontology 31: 626-640. Maryanska, T., R.E. Chapman, and D.B. Weishampel. 2004. Pachycephalosauria. In D.B. Weishampel, P. Dodson, and H. Osmólska (editors), The Dinosauria: 464-477. Berkeley: University of California Press.
Morschhauser, E.M., and M.C. Lamanna. 2013. A reevaluation of the hyoid bones of Protoceratops andrewsi Granger and Gregory, 1923 (Ornithischia: Ceratopsia) and a review of hyoid elements in ornithischian dinosaurs. Annals of Carnegie Museum 81: 247-255.

Nabavizadeh, A., and D.B. Weishampel. 2016. The predentary bone and its significance in the evolution of feeding mechanisms in ornithischian dinosaurs. Anatomical Record 299: 1358-1388.

Nesbitt, S.J. 2011. The early evolution of archosaurs: relationships and the origin of major clades. Bulletin of the American Museum of Natural History 352: 1-292.

Nesbitt, S.J., A.H. Turner, and J.C. Weinbaum. 2012. A survey of skeletal elements in the orbit of Pseudosuchia and the origin of the crocodylian palpebral. Earth and Environmental Science Transactions of the Royal Society of Edinburgh 103: 365-381.

Norell, M.A., and D.E. Barta. 2016. A new specimen of the ornithischian dinosaur Haya griva, cross-Gobi geologic correlation, and the age of the Zos Canyon beds. American Museum Novitates 3851: 1-19.

Norell, M., and P.J. Makovicky. 1997. Important features of the dromaeosaur skeleton: information from a new specimen. American Museum Novitates 3215: 1-28.

Norell, M.A., A.M. Balanoff, D.E. Barta, and G.M. Erickson. 2018. A second specimen of Citipati osmolskae associated with a nest of eggs from Ukhaa Tolgod, Omnogov Aimag, Mongolia. American Museum Novitates 3899: 1-44.

Norman, D.B. 1980. On the ornithischian dinosaur Iguanodon bernissartensis from the Lower Cretaceous of Bernissart (Belgium). Memoire de l'Institut Royal des Sciences Naturelles de Belgique 178: 1-105.

Norman, D.B. 2004. Basal Iguanodontia. In D.B. Weishampel, P. Dodson, and H. Osmólska (editors), The Dinosauria: 413-437. Berkeley: University of California Press.

Norman, D.B., H.-D. Sues, L.M. Witmer, and R.A. Coria. 2004. Basal Ornithopoda. In D.B. Weishampel, P. Dodson, and H. Osmólska (editors), The Dinosauria: 393-412. Berkeley: University of California Press.

Norman, D.B., A.W. Crompton, R.J. Butler, L.B. Porro, and A.J. Charig. 2011. The Lower Jurassic ornithischian dinosaur Heterodontosaurus tucki Crompton \& Charig, 1962: cranial anatomy, functional morphology, taxonomy, and relationships. Zoological Journal of the Linnean Society 163: 182-276. 
Novas, F.E., A.V. Cambiaso, and A. Ambrosio. 2004. A new basal iguanodontian (Dinosauria, Ornithischia) from the Upper Cretaceous of Patagonia. Ameghiniana 41: 75-82.

Oelrich, T.M. 1956. The anatomy of the head of Ctenosaura pectinata (Iguanidae). Miscellaneous Publications Museum of Zoology, University of Michigan 94: $1-122$.

Organ, C.L. 2006. Thoracic epaxial muscles in living archosaurs and ornithopod dinosaurs. Anatomical Record 288A: 782-793.

Ostrom, J.H. 1961. Cranial morphology of the hadrosaurian dinosaurs of North America. Bulletin of the American Museum of Natural History 122 (2): 33-186.

Parker, W.K. 1868. A monograph on the structure and development of the shoulder girdle and sternum in the Vertebrata. London: Ray Society.

Parks, W.A. 1926. Thescelosaurus warreni, a new species of orthopodous dinosaur from the Edmonton Formation of Alberta. University of Toronto Studies, Geological Series 21: 1-42.

Peng, G. 1992. Jurassic ornithopod Agilisaurus louderbacki (Ornithopoda: Fabrosauridae) from Zigong, Sichuan, China. Vertebrata PalAsiatica 30: 39-51.

Pol, D., O.W.M. Rauhut, and M. Becerra. 2011. A Middle Jurassic heterodontosaurid dinosaur from Patagonia and the evolution of heterodontosaurids. Naturwissenschaften 98: 369-379.

Porro, L.B., L.M. Witmer, and P.M. Barrett. 2015. Digital preparation and osteology of the skull of Lesothosaurus diagnosticus (Ornithischia: Dinosauria). PeerJ 3: e1494.

Rauhut, O.W. 2003. The interrelationships and evolution of basal theropod dinosaurs. Special Papers in Palaeontology 69: 1-213.

Rogers, K.C., M. Whitney, M. D'Emic, and B. Bagley. 2016. Precocity in a tiny titanosaur from the Cretaceous of Madagascar. Science 352: 450-453.

Romer, A.S. 1927. The pelvic musculature of ornithischian dinosaurs. Acta Zoologica 8: 225-275.

Romer, A.S. 1956. Osteology of the reptiles. Chicago: University of Chicago Press.

Rozadilla, S., F. L. Agnolín, and F. E. Novas. 2019. Osteology of the Patagonian ornithopod Talenkauen santacrucensis (Dinosauria, Ornithischia). Journal of Systematic Palaeontology 17: 2043-2089.

Rozadilla, S., P. Cruzado-Caballero, and J. O. Calvo. 2020. Osteology of ornithopod Macrogryphosaurus gondwanicus (Dinosauria, Ornithischia) from the Upper Cretaceous of Patagonia, Argentina. Cretaceous Research 108: 104311.
Russell, L.S. 1940. The sclerotic ring in the Hadrosauridae. Contributions of the Royal Ontario Museum of Palaeontology 3: 1-7.

Salgado, L., R.A. Coria, and S.E. Heredia. 1997. New Materials of Gasparinisaura cincosaltensis (Ornithischia, Ornithopoda) from the Upper Cretaceous of Argentina. Journal of Paleontology 71: 933-940.

Salgado, L., et al. 2017. A new primitive neornithischian dinosaur from the Jurassic of Patagonia with gut contents. Scientific Reports 7: 42778.

Santa Luca, A.P. 1980. The postcranial skeleton of Heterodontosaurus tucki (Reptilia: Ornithischia) from the Stormberg of South Africa. Annals of the South African Museum 79: 159-211.

Scheetz, R.D. 1999. Osteology of Orodromeus makelai and the phylogeny of basal ornithopod dinosaurs. Ph.D., Montana State University, Bozeman, 186 pp.

Schindelin, J., et al. 2012. Fiji: an open-source platform for biological-image analysis. Nature Methods 9: 676-682.

Sereno, P. 2012. Taxonomy, morphology, masticatory function and phylogeny of heterodontosaurid dinosaurs. ZooKeys 226: 1-225.

Sereno, P.C. 1991. Lesothosaurus, "Fabrosaurids," and the early evolution of Ornithischia. Journal of Vertebrate Paleontology 11: 168-197.

Sereno, P.C., R.N. Martínez, and O.A. Alcober. 2012. Osteology of Eoraptor lunensis (Dinosauria, Sauropodomorpha). Journal of Vertebrate Paleontology 32: 83-179.

Sobral, G., C.A. Hipsley, and J. Mueller. 2012. Braincase redescription of Dysalotosaurus lettowvorbecki (Dinosauria, Ornithopoda) based on computed tomography. Journal of Vertebrate Paleontology 32: 1090-1102.

Sues, H.-D. 1980. Anatomy and relationships of a new hypsilophodontid dinosaur from the Lower Cretaceous of North America. Palaeontographica Abteilung A 51-72.

Thomas, D.A. 2015. The cranial anatomy of Tenontosaurus tilletti Ostrom, 1970 (Dinosauria, Ornithopoda). Palaeontologia Electronica 18: 1-99.

Thulborn, R.A. 1972. The post-cranial skeleton of the Triassic ornithischian dinosaur Fabrosaurus australis. Palaeontology 15: 29-60.

Thulborn, R.A. 1974. A new heterodontosaurid dinosaur (Reptilia: Ornithischia) from the Upper Triassic red beds of Lesotho. Zoological Journal of the Linnean Society 55: 151-175.

Tsuihiji, T. 2010. Reconstructions of the axial muscle insertions in the occipital region of dinosaurs: evaluations of past hypotheses on Marginocephalia and 
Tyrannosauridae using the extant phylogenetic bracket approach. Anatomical Record 293: 1360-1386.

Tsuihiji, T., et al. 2011. Cranial osteology of a juvenile specimen of Tarbosaurus bataar (Theropoda, Tyrannosauridae) from the Nemegt Formation (Upper Cretaceous) of Bugin Tsav, Mongolia. Journal of Vertebrate Paleontology 31: 497-517.

Upchurch, P. 1995. The evolutionary history of sauropod dinosaurs. Philosophical Transactions of the Royal Society London B 349: 365-390.

Varricchio, D.J., A.J. Martin, and Y. Katsura. 2007. First trace and body fossil evidence of a burrowing, denning dinosaur. Proceedings of the Royal Society B, Biological Sciences 274: 1361-1368.

Weishampel, D. 1984. Evolution of jaw mechanisms in ornithopod dinosaurs. Advances in Anatomy, Embryology, and Cell Biology 87: 1-109.

Weishampel, D.B., C. Jianu, Z. Csiki, and D.B. Norman. 2003. Osteology and phylogeny of Zalmoxes (n. g.), an unusual Euornithopod dinosaur from the latest Cretaceous of Romania. Journal of Systematic Palaeontology 1: 65-123.

Werning, S. 2012. The ontogenetic osteohistology of Tenontosaurus tilletti. PLoS ONE 7: e33539.

Wilson, J. A., D. Pol, A. B. Carvalho, and H. Zaher. 2016. The skull of the titanosaur Tapuiasaurus macedoi (Dinosauria: Sauropoda), a basal titanosaur from the Lower Cretaceous of Brazil. Zoological Journal of the Linnean Society 178: 611-662.

Witmer, L.M. 1990. The craniofacial air sac system of Mesozoic birds (Aves). Zoological Journal of the Linnean Society 100: 327-378.

Woodward, H.N., E.A. Freedman Fowler, J.O. Farlow, and J.R. Horner. 2015. Maiasaura, a model organisim for extinct vertebrate population biology: a large sample statistical assessment of growth dynamics and survivorship. Paleobiology 41: 503-527.

Woodward, H.N., T.H. Rich, A. Chinsamy, and P. Vickers-Rich. 2011. Growth dynamics of Australia's polar dinosaurs. PLoS ONE 6: e23339.

Woodward, H.N., T.H. Rich, and P. Vickers-Rich. 2018. The bone microstructure of polar "hypsilophodontid" dinosaurs from Victoria, Australia. Scientific Reports 8: 1162.

Yates, A.M. 2005. A new theropod dinosaur from the Early Jurassic of South Africa and its implications for the early evolution of theropods. Palaeontologia Africana 41: 105-122.

You, H., and P. Dodson. 2004. Basal Ceratopsia. In D.B. Weishampel, P. Dodson, and H. Osmólska (editors),
The Dinosauria: 478-493. Berkeley: University of California Press.

Zan, S., J. Chen, L. Jin, and T. Li. 2005. A primitive ornithopod from the Early Cretaceous Quantou Formation of central Jilin, China. Vertebrata PalAsiatica 43: 182-193.

Zheng, W., X. Jin, M. Shibata, Y. Azuma, and F. Yu. 2012. A new ornithischian dinosaur from the Cretaceous Liangtoutang Formation of Tiantai, Zhejiang Province, China. Cretaceous Research 34: 208-219.

Zheng, X.-T., H.-L. You, X. Xu, and Z.-M. Dong. 2009. An Early Cretaceous heterodontosaurid dinosaur with filamentous integumentary structures. Nature 458: 333-336. 


\section{APPENDIX 1}

\section{Anatomical AbBreviations}

\begin{tabular}{|c|c|c|c|}
\hline $4 \mathrm{t}$ & fourth trochanter & il & ilium \\
\hline ae & articulation for ectopterygoid (on palatine) & ip & ischial peduncle \\
\hline ai & axial intercentrum & is & ischium \\
\hline am & articulation for maxilla (on palatine) & j & jugal \\
\hline an & angular & la & lacrimal \\
\hline aof & antorbital fenestra & lco & lateral condyle \\
\hline apa & ascending process of astragalus & lil & left ilium \\
\hline ar & articular & lpe & lateral process of ectopterygoid \\
\hline as & astragalus & lt & lesser trochanter \\
\hline aspo & anterior supraorbital & ltar & lateral tarsal \\
\hline at & atlas & $\mathrm{mc}$ & metacarpal \\
\hline ati & atlantal intercentrum & mco & medial condyle \\
\hline $\mathrm{ax}$ & axis & $\mathrm{mr}$ & mandibular ramus of pterygoid \\
\hline bf & brevis fossa & $\mathrm{mt}$ & metatarsal \\
\hline bo & basioccipital & mtar & medial tarsal \\
\hline bpro & boss for articulation with proatlas & $\mathrm{mx}$ & maxilla \\
\hline bptp & basipterygoid process & $\mathrm{mxf}$ & maxillary fenestra \\
\hline bs & basisphenoid & $\mathrm{n}$ & nasal \\
\hline c & cervical vertebra & $\mathrm{nf}$ & nutrient foramina \\
\hline $\mathrm{ca}$ & caudal vertebra & op & odontoid process \\
\hline $\mathrm{cal}$ & calcaneum & ot & ossified tendons \\
\hline $\mathrm{cb}$ & ceratobranchial & $\mathrm{p}$ & phalanx \\
\hline $\mathrm{cc}$ & cnemial crest & pa & parietal \\
\hline $\mathrm{ch}$ & chevron & par & prearticular \\
\hline co & coracoid & $\mathrm{pd}$ & predentary \\
\hline$c p$ & cultriform process & $\mathrm{pf}$ & prefrontal \\
\hline $\mathrm{cr}$ & cervical rib & pif & pituitary fossa \\
\hline d & dorsal vertebra & plr & palatine ramus of pterygoid \\
\hline de & dentary & $\mathrm{pm}$ & premaxilla \\
\hline eoc & exoccipital & po & postorbital \\
\hline ept & ectopterygoid & pop & paraoccipital process \\
\hline $\mathrm{f}$ & frontal & $\mathrm{pp}$ & pubic peduncle \\
\hline $\mathrm{fe}$ & femur & ppf & postpalatine fenestra \\
\hline fi & fibula & prp & prepubic process \\
\hline ga & gastroliths & pspo & posterior supraorbital \\
\hline gt & greater trochanter & $\mathrm{pt}$ & pterygoid \\
\hline gv & groove & $\mathrm{pu}$ & pubis \\
\hline hu & humerus & $q$ & quadrate \\
\hline
\end{tabular}




\section{APPENDIX 1 continued}

$\begin{array}{ll}\text { qj } & \text { quadratojugal } \\ \text { qjf } & \text { quadratojugal foramen } \\ \text { qw } & \text { quadrate wing of pterygoid } \\ \text { ra } & \text { radius } \\ \text { ril } & \text { right ilium } \\ \text { s } & \text { sacral vertebra } \\ \text { sa } & \text { surangular } \\ \text { sc } & \text { scapula } \\ \text { sk } & \text { skull } \\ \text { so } & \text { supraoccipital } \\ \text { sp } & \text { splenial } \\ \text { sq } & \text { squamosal } \\ \text { st } & \text { sternum } \\ \text { sta } & \text { stapes } \\ \text { ti } & \text { tibia } \\ \text { ul } & \text { ulna } \\ \text { vk } & \text { ventral keel (on vomer) } \\ & \\ \end{array}$

\section{APPENDIX 2}

Character List from Madzia et al. (2018) for Phylogenetic Analyses

Nexus files for the three phylogenetic analyses and STL files of surface scanned specimens are available in the online supplement (https://doi.org/10.5531/ sd.sp.47).

1. Preorbital skull length less than or equal to $50 \%$ total skull length (0), greater than $50 \%$ (1).

2. Predentary bone absent (0), present (1).

3. Rostral bone absent $(0)$, present (1).

4. Oral margin of the premaxilla smooth (0), denticulate (1).

5. Lateral surface of the oral margins of the premaxillae flat (0), everted (1).

6 . Ventral margin of the premaxillalevel with the maxillary toothrow (0), ventrally deflected (1).

7. Anteriormost premaxillary tooth positioned at the anterior margin of the premaxilla (0), inset at least the width of one tooth crown (1).

8. Diastema between premaxilla and maxilla absent (0), present (1).

9. Diastema between premaxilla and maxilla flat (0), arched (1).

10. Anterior premaxillary foramen absent (0), present (1).

11. Premaxillary border of internal nares present $(0)$, absent (1).

12. Anterodorsal surface of the premaxilla smooth (0), highly rugose (1).

13. Premaxillary posterolateral process does not exclude maxilla from nasal margin (0), does exclude maxilla from nasal margin (1).

14. Concavity within the posterior end of the premaxilla, near lateral margin, for receipt of the anterolateral boss of the maxilla absent (0), present (1).

15. Overlap of the dorsal process of the premaxilla onto the rostral process of the nasal absent (0), present (1).

16. Contact between premaxilla and lacrimal absent (0), present (1).

17. Premaxillary sulcus on the anterior process of the maxilla absent (0), present (1).

18. "Special foramina" medial to dentary and maxillary toothrows absent (0), present (1).

19. Buccal emargination on the maxilla absent (0), present (1).

20. Buccal emargination of maxilla consists of a gradual and shallow beveling of the ventrolateral surface of 
the maxilla (0), consists of a prominent ridge on the lateral surface of the maxilla (1).

21. Notch in maxilla for the lacrimal absent (0), present (1).

22. Fossa situated low along the boundary between the premaxilla and the maxilla absent (0), present (1).

23. Supraorbital absent (0), one supraorbital present (1), two or more supraorbitals present (2).

24. Supraorbital free and projects into orbit from contact with lacrimal and prefrontal (0), supraorbital incorporated into the orbital margin (1).

25. Supraorbital(s) extend across at least $71 \%$ of the maximum anteroposterior length of the orbit (0), $70 \%$ or less (1).

26. Greatest posterior expanse of the jugal greater than $25 \%$ height of skull (0), less than $25 \%$ (1).

27. Anterior process of the jugal straight (0), curved (1).

28. Anterior process of jugal situated dorsal to the maxilla (0), anterior process of jugal inserts into the maxilla (1).

29. Jugal barely contacts lacrimal (0), jugal touches lacrimal with more contact (1), butt joint between jugal and lacrimal (2).

30. Jugal contributes to the antorbital fenestra ( 0$)$, does not reach antorbital fenestra (1).

31. Contact between jugal and postorbital faces anteriorly (0), contact faces partially laterally (1), postorbital inserts into a socket in the jugal (2).

32. Jugal dorsoventrally deeper than mediolaterally broad (0), broader than deep (1).

33. Jugal forms part of the posterior margin the infratemporal fenestra ( 0 ), only forms the ventral margin (1).

34. The anteroventral corner of the infratemporal fenestra, formed by the jugal, consists of an oblique or right angle (0), anteroventral corner of the infratemporal fenestra consists of an acute angle (1).

35. Contact between the jugal and quadratojugal consists of a butt or high-angle scarf joint ( 0 ), the jugal overlaps the lateral surface of the quadratojugal posterodorsally and the medial surface posteroventrally (1).

36. Jugal does not contact quadrate (0), jugal does contact quadrate (1).

37. Jugal or quadratojugal contacts the quadrate near or slightly above the distal end ( 0 ), contacts positioned well above the distal end (1).

38. No boss or ornamentation present on lateral surface of the jugal (0), lateral surface of jugal ornamented, but no boss present (1), presence of a low boss on the lateral surface of the jugal (2), presence of a tall, posteriorly projecting boss on the lateral surface of the jugal (3).

39. Maxillary process on the medial side of the jugal is medially projected and modestly arched (0), maxillary process on the medial side of the jugal is straight and grooved (1), maxillary process on the medial side of the jugal is anteromedially projected and arched (2).

40. Ectopterygoid facet on the medial surface of the jugal consists of an abbreviated, deep groove (0), ectopterygoid facet consists of a rounded scar (1).

41. Quadratojugal foramen absent (0), present (1).

42. Dorsal process of the quadratojugal contacts the descending process of the squamosal (0), dorsal process of the quadratojugal does not contact the descending process of the squamosal (1).

43. Quadratojugal anteroposteriorly long and dorsoventrally short (0), quadratojugal anteroposteriorly short and dorsoventrally tall (1).

44. Quadratojugal contacts the quadrate along greater than $50 \%$ the total length of the quadrate (0), quadratojugal contacts the quadrate along less than $50 \%$ the total length of quadrate (1).

45. Proximal head of the quadrate recurved posteriorly (0), proximal head of quadrate straight (1).

46. Ventral portion of the quadrate shaft oriented vertically or anteroventrally angled (0), ventral portion of the quadrate shaft posteroventrally angled (1).

47. Body of the quadrate leans posteriorly (0), body of quadrate oriented vertically (1), body of quadrate leans anteriorly (2).

48. Jugal wing of the quadrate moderately developed (0), jugal wing of the quadrate shortened (1).

49. Ventral extent of the jugal wing of the quadrate positioned at or near the distal end of the quadrate $(0)$, ventral extent of the jugal wing of the quadrate positioned above the distal end of the quadrate (1).

50. Pit in lateral side of quadrate at the base of the jugal wing present (0), absent (1).

51. Quadrate notch (tiny foramen between jugal wing of quadrate and quadratojugal) absent (0), present (1).

52. Distal condyles of the quadrate dorsomedially sloped or horizontally oriented (0), distal condyles of the quadrate dorsolaterally sloped (1).

53. The pterygoid wing of the quadrate arises at the dorsal head of the quadrate (0), pterygoid wing of the quadrate arises below the dorsal head of the quadrate (1).

54. Pterygoid wing of quadrate consists of a large, anteromedially extending fan of bone (0), pterygoid wing of the quadrate small (1). 
55. Groove on the base of the posterior side of the pterygoid wing of the quadrate absent $(0)$, present (1).

56. Length of ventral process of the squamosal less than $30 \%$ the total length of the quadrate $(0)$, length of the ventral process of the squamosal greater than $30 \%$ the total length of the quadrate (1).

57. Paroccipital process oriented horizontally and slightly widened distally (0), distal end of paroccipital process "pendant shaped" (1).

58. Parietosquamosal shelf absent (0), present (1).

59. Orbital edge of postorbital forms a smooth, continuous arc (0), orbital edge of postorbital possesses an anteriorly directed inflation into orbit (1).

60. Orbital margin of postorbital smooth (0), orbital margin striated and rugose (1).

61. Postorbital nonrobust (0), postorbital robust (1).

62. Synovial socket for the head of the laterosphenoid positioned along the frontal-postorbital contact (0), synovial socket for the head of the laterosphenoid positioned only in the postorbital (1), synovial socket for the head of the laterosphenoid positioned only in the frontal (2), no synovial joint for the laterosphenoid present (3).

63. Greater than $25 \%$ of the frontal length participates in the orbital margin (0), less than $25 \%$ of the frontal length participates in the orbital margin.

64. Frontals extend over the entire orbit (0), frontals positioned only over the posterior half of orbit (1).

65 . Frontals arched over orbit $(0)$, frontals dorsally flattened (1).

66. Combined width of frontals greater than the total length of the frontals $(0)$, combined width of the frontals less than the total length of the frontals (1).

67. Total length of frontals between $120 \%$ and $60 \%$ the total length of the nasals ( 0$)$, total length of frontal less than $60 \%$ the total length of the nasals (1).

68. Length of the oral margin of the predentary less than the length of the oral margin of the premaxilla (0), length of the oral margin of the predentary equal to or greater than the length of the oral margin of the premaxilla (1).

69. Anterior tip of predentary pointed (0), anterior tip rounded (1).

70. Oral margin of the predentary smooth $(0)$, oral margin denticulate (1).

71. Ventral process of the predentary present (0), ventral process of the predentary very reduced or absent (1).

72. One ventral process of the predentary (0), two ventral processes of the predentary (1).
73. Dentary symphysis V-shaped (0), dentary symphysis spout shaped (1).

74. Anteriormost tip of dentary positioned within the dorsal $1 / 3$ of the dentary $(0)$, anteriormost tip positioned near midheight of the dentary (1), anteriormost tip positioned within the lower $1 / 3$ of the dentary (2), anteriormost tip curves ventrally below ventral margin of the dentary (3), anteriormost tip anterodorsally curved and positioned higher than the base of the dentary toothrow (4).

75. Dorsal and ventral margins of the dentary converge rostrally (0), dorsal and ventral margins of the dentary parallel (1).

76. Medial surface of the dentary straight $(0)$, medial surface of the dentary medially arched (1).

77. Dentary depth just anterior to the rising coronoid process $20 \%$ or less the total length of the dentary (0), dentary depth $21 \%$ or more the total length of the dentary (1).

78. Dentary toothrow straight in lateral view (0), dentary toothrow sinuous in lateral view (1).

79. Coronoid process absent or weakly developed (0), coronoid process present (1).

80. Dentary does not contribute to the coronoid process (0), dentary does contribute to the coronoid process (1).

81. The posterior end of toothrow ends anterior to coronoid process $(0)$, the posterior end of tooth the row is shrouded by the coronoid process in lateral view (1).

82 . Coronoid process inconspicuous $(0)$, coronoid process subtriangular (1), coronoid process subrectangular (2), coronoid process dorsally elongated with a lobe-shaped distal expansion (3).

83. The length of the mandible posterior to the coronoid process is $36 \%$ or greater the total length of mandible (0), the postcoronoid length of the mandible is between 25 and $35 \%$ the total length of the mandible (1), the postcoronoid length of the mandible is less than $25 \%$ the total length of the mandible (2).

84. Dorsal margin of the surangular convex or diagonal (0), dorsal margin of the surangular concave (1).

85. Surangular foramen absent (0), present (1).

86. Ridge or process on lateral surface of surangular, anterior to the jaw suture absent (0), a strong, anteroposteriorly extending ridge present (1), a dorsally directed, fingerlike process present (2).

87. Distal condyles of the quadrate subequal (0), medial distal condyle larger (1), lateral distal condyle larger (2).

88. Maximum length of external nares less than $15 \%$ basal skull length (0), maximum length of external nares greater than 15\% basal skull length (1). 
89. External nares positioned close to the buccal margin and below the level of the orbit (0), external nares positioned higher than the maxilla (1).

90. Antorbital fenestra present (0), absent (1).

91. Antorbital fossa rounds smoothly onto maxilla along some part of its margin (0), antorbital fossa sharply defined or extended as a secondary lateral wall enclosing the fossa (1).

92. Maxillary fenestra absent (0), present (1).

93. Antorbital fossa triangular (0), antorbital fossa ovate or circular (1).

94. External opening of the antorbital fossa present and greater than $10 \%$ basal skull length $(0)$, external opening of the antorbital fossa present and less than $10 \%$ basal skull length (1), external opening of the antorbital fossa absent (2).

95. Lower margin of the orbit circular (0), lower margin of the orbit subrectangular (1).

96. Ventral edge of the infratemporal fenestra extends to or below the ventral margin of the orbit $(0)$, ventral edge of the infratemporal fenestra positioned well above the ventral margin of the orbit (1).

97. External mandibular fenestra present $(0)$, absent (1).

98. Angle between the base and long axis of the braincase greater than $35^{\circ}(0)$, angle less than $35^{\circ}(1)$.

99. Median ridge on floor of the braincase absent $(0)$, present (1).

100. Basioccipital and exoccipital contribute to the occipital condyle (0), exoccipital excluded from occipital condyle (1).

101. Nuchal crest on the supraoccipital absent (0), present (1).

102. Supraoccipital contributes to greater than $5 \%$ of the margin of the foramen magnum (0), supraoccipital contributes to less than $5 \%$ of the margin of the foramen magnum (1), supraoccipital excluded from the margin of the foramen magnum (2).

103. Posttemporal foramen positioned at the boundary between the parietals and the paroccipital process (0), posttemporal foramen positioned entirely within the opisthotic (1), posttemporal foramen positioned entirely within the squamosal (2).

104. Posttemporal foramen consists of an enclosed foramen (0), posttemporal foramen consists of a dorsally open groove (1).

105. Ventral keel on basioccipital absent (0), present (1).

106. Floor of basioccipital flat (0), arched (1).

107. Basioccipital tubera lower than basisphenoid (0), basioccipital tubera level with basisphenoid (1).
108. Foramen magnum occupies over $30 \%$ of the dorsal margin of the occipital condyle $(0)$, foramen magnum occupies between $30 \%$ and $20 \%$ of the dorsal margin of the occipital condyle (1), foramen magnum occupies less than $20 \%$ of the dorsal margin of the occipital condyle (2).

109. Length of basisphenoid (from base of the parasphenoid process to the posterior edge of the basisphenoid) less than the length of basioccipital (0), basisphenoid and basioccipital subequal in length (1), length of basisphenoid greater than the length of the basioccipital (2).

110. Foramen for cranial nerve $\mathrm{V}$ notches anteroventral edge of prootic (0), foramen for cranial nerve $\mathrm{V}$ completely enclosed within the prootic (1).

111. Premaxillary teeth present (0), absent (1).

112. Six teeth present in each premaxilla $(0)$, between four and five teeth present in each premaxilla (1), one or less teeth present in each premaxilla (2).

113. Enlarged anterior canine tooth in dentary absent (0), present (1).

114. Ridges absent on dentary teeth, only simple denticles present $(0)$, at least some denticles confluent with ridges that extend to base of crown on dentary teeth (1).

115. Apex of the maxillary teeth centrally placed (0), apex of maxillary teeth placed posterior of center (1).

116. Premaxillary teeth recurved, transversely flattened, constricted at the base $(0)$, premaxillary teeth straight, subcylindrical, and unconstructed at the base. (1).

117. Space present between the roots and crowns of adjacent maxillary teeth (0), lack of space between crowns of adjacent maxillary teeth up through the occlusional margin (1), lack of space between roots and crowns of adjacent maxillary teeth (2), no space between crowns within each tooth position within the maxilla (3).

118. Apical ridge on the dentary teeth placed centrally or anterior of center $(0)$, apical ridge of the dentary teeth placed posterior of center (1).

119. Maxillary tooth roots straight in anterior or posterior view (0), maxillary tooth roots curved in anterior or posterior view (1).

120. Distinct neck present below the crown of the maxillary teeth (0), maxillary crown tapers to root (1).

121. Maxillary teeth independently occlude (0), maxillary teeth share a continuous occlusional surface (1).

122. Lingual surface of maxillary teeth concave (0), lingual surface convex (1), lingual surface flat (2). 
123. Distribution of enamel on maxillary and dentary teeth roughly equal on both sides (0), enamel primarily restricted to one side on maxillary and dentary teeth (1).

124. Ridges present on both sides of dentary crowns (0), ridges limited to one side of dentary crowns (1).

125. Maxillary crowns low and spadelike, rectangular, or triangular (0), maxillary crowns high and diamond shaped (1), maxillary crowns laterally flattened and posteriorly recurved (2), maxillary crowns conical (3).

126. Twelve or fewer teeth present in each maxilla (0), between 13 and 19 teeth present in each maxilla (1), 20 or more teeth present in each maxilla (2).

127. Maxillary teeth possess a smooth face with simple denticles (0), maxillary teeth possess ridges confluent with denticles that extend to base of crown (1).

128. All ridges on maxillary teeth equally prominent (0), one ridge on maxillary teeth more prominent than the rest (1).

129. Cingulum on maxillary teeth present (0), cingulum on maxillary teeth absent (1).

130. Cingulum on dentary teeth absent (0), cingulum on dentary teeth present (1).

131. Maxillary teeth positioned near the lateral margin (0), maxillary teeth inset medially (1).

132. Maxillary crown height less than $50 \%$ length (0), maxillary crown height between $50 \%$ and $90 \%$ crown length (1), maxillary crown height subequal to the crown length (2), maxillary crown height between $110 \%$ and $150 \%$ crown length (3), maxillary crown height greater than $150 \%$ crown length (4).

133. Dentary crowns less than $50 \%$ higher than mesiodistally wide (0), dentary crowns greater than $50 \%$ higher than mesiodistally wide (1).

134. Denticles extend beyond apical third of maxillary and dentary tooth crowns (0), denticles restricted to apical third of maxillary and dentary tooth crowns (1).

135. Dentary tooth roots straight in anterior or posterior view (0), dentary tooth roots curved in anterior or posterior view (1).

136. Dentary crowns rectangular, triangular, or leaf shaped (0), dentary teeth lozenge shaped (1), dentary teeth laterally flattened, posteriorly recurved (2), dentary teeth conical (3).

137. Thirteen or fewer dentary teeth present (0), between 14 and 17 dentary teeth present (1), 18 or more dentary teeth present (2).

138. Fewer than 10 ridges present on dentary teeth (0), 10 or more ridges present on dentary teeth (1).
139. All ridges on the dentary teeth equally prominent (0), one ridge more prominent than the rest on the dentary teeth (1).

140. Anterior two dentary teeth similar in morphology to more posterior dentary teeth (0), anterior two dentary teeth lack denticles, first tooth strongly reduced (1).

141. Fewer than 10 cervical vertebrae (0), 10 or more cervical vertebrae (1).

142. Cervical vertebrae plateocoelous or amphicoelous (0), opisthocoelous (1).

143. Ventral surface of the cervical vertebrae rounded $(0)$, presence of a broad, flattened keel on the ventral surface of the cervical vertebrae (1), presence of a sharp ventral keel on the ventral surface of the cervical vertebrae (2).

144. Anterior cervical centra less than $1.5 \times$ longer than tall (0), length of anterior cervical centra equal or greater than $1.5 \times$ longer than tall (1).

145. Epipophyses on anterior cervical three present (0), absent (1).

146. Dorsal neural spines arise anteriorly or are centered over centrum (0), dorsal neural spines posteriorly positioned on centrum (1).

147. Fourteen or fewer dorsal vertebrae present (0), 15 dorsal vertebrae present (1), 16 dorsal vertebrae present (2), 17 or more dorsal vertebrae present (3).

148. Sacrum composed of three or fewer fused vertebral centra (0), sacrum composed of between four and five fused vertebral centra (1), sacrum composed of six fused vertebral centra (2), sacrum composed of seven or more fused vertebra centra (3).

149. Sacral neural spines less than $2 \times$ the height of the sacral centra $(0)$, sacral neural spines $2-2.5 \times$ the height of the sacral centra (1), sacral neural spines greater than $2.5 \times$ the height of the sacral centra (2).

150. Sacral neural spines lean posteriorly (0), sacral neural spines lean anteriorly (1).

151. Height of neural spine on proximal caudal vertebrae less than $1.5 \times$ taller than the height of the centrum (0), height of neural spine greater than $1.5 \times$ taller than the height of the centrum (1).

152. Caudal neural spines positioned entirely over their respective caudal centra (0), caudal neural spines extend beyond their own centrum (1).

153. Caudal ribs positioned entirely on caudal centra (0), caudal ribs positioned along neurocentral suture (1), caudal ribs positioned on neural arch (2).

154. First caudal vertebra bears the longest caudal rib (0), longest caudal rib positioned posterior to first caudal vertebra (1). 
155. Axial epiphyses present at least vestigially (0), axial epiphyses absent (1).

156. Transition from tuberculum and capitulum of dorsal ribs from vertical to near horizontal occurs between dorsal vertebrae 2 and $4(0)$, transition occurs between dorsal vertebrae 5 and 6 (1), transition occurs between dorsal vertebrae 6 and 8 (2).

157. Partial ossification of the sternal segments of the cranial dorsal ribs absent (0), present (1).

158. Scapular spine on the anterodorsal corner of the scapula low or broad (0), scapular spinesharp and pronounced (1).

159. Minimum thickness of scapular neck less than $20 \%$ maximum length of the scapula (0), minimum width of neck greater than $20 \%$ maximum length of the scapula (1).

160. Width of coracoid less than $60 \%$ the length of the coracoid (0), width of coracoids between $61 \%$ and $100 \%$ the length of the coracoid (1), width of the coracoid greater than the length of the coracoid (2).

161. Coracoid foramen enclosed within the coracoids (0), coracoid foramen open along coracoid-scapula articular contact surface (1).

162. Length of sternal process of the coracoids (measured from the tip of the sternal process of the coracoid to the base of the coracoid notch less than $70 \%$ the width of the sternal process measured at the level of the base of the coracoids notch) (0), length of sternal process greater than $70 \%$ the width of the sternal process (1).

163. Ovoid fossa positioned anteroventral to the glenoid fossa on the coracoid absent (0), present (1).

164. Sternal plates crescent shaped (0), sternal plates hatchet shaped (1), sternal plates expanded along the anterior and posterior ends and constricted in the middle (2).

165. Humerus longer than or subequal to scapula (0), scapula longer than humerus (1).

166. Total forelimb length [measured from the head of the humerus to tip of the manus] longer than $40 \%$ the total length of the hind limb [measured from the head of the femur to tip of the pes] (0), total forelimb length equal to or less than $40 \%$ the total length of the hind limb (1).

167. Shaft of the humerus straight (0), shaft of the humerus exhibits at least a modest caudal flexure at the level of the deltopectoral crest (1).

168. Deltopectoral crest rounded in lateral view (0), deltopectoral crest angular in lateral view (1), deltopectoral crest inconspicuous (2).
169. Olecranon process low (0), olecranon process moderately developed (1), olecranon process well developed (2).

170. Cross-sectional shape of the ulna at midshafttriangular or oval (0), cross-sectional shape of the ulna at midshaft cylindrical (1).

171. Shaft of the ulna straight (0), shaft of the ulna bowed (1).

172. Minimal radial width less than $10 \%$ the length of the radius $(0)$, minimal radial width greater than $10 \%$ the length of the radius (1).

173. Carpus unfused (0), carpus fused (1).

174. Metacarpal I greater than $50 \%$ the length of metacarpal ii (0), metacarpal I less than 50\% the length of metacarpal ii (1).

175. Manual digit I oriented less than $25^{\circ}$ from digit iii (0), manual digit I oriented between $25^{\circ}$ and $60^{\circ}$ from digit iii (1), manual digit I oriented at an angle greater than $60^{\circ}$ from digit iii (2).

176. Ungual of manual digit I clawlike (0), ungual subconical (1), ungual absent (2).

177. First phalanx of manual digits ii-iv less than twice the size of second phalanx (0), first phalanx greater than $2 \times$ the size of second phalanx (1).

178. Unguals on manual digits ii and iii longer than wide (0), unguals on manual digits ii and iii wider than long (1).

179. Four phalanges present in manual digit iii (0), three phalanges present in manual digit iii (1).

180. Three phalanges present in manual digit IV (0), two phalanges present in manual digit IV (1), one phalanx present in manual digit iv (2).

181. Two phalanges present in manual digit $\mathrm{V}(0)$, one phalanx present in manual digit $\mathrm{V}(1)$, phalanges absent in manual digit $\mathrm{V}(2)$.

182. Acetabulum on ilium normal to high (0), acetabulum on ilium short to long (1).

183. Ventral acetabular flange on the ilium present (0), absent (1).

184. Supraacetabular rim on the ilium weakly developed or absent (0), supraacetabular rim on the ilium strongly developed (1).

185. Dorsal margin of the ilium straight to slightly convex in lateral view (0), dorsal margin of the ilium sinuous in lateral view (1).

186. External surface of the preacetabular process of the ilium laterally facing and roughly in the same plane as the body of the ilium (0), external surface of the preacetabular process of the ilium twisted about its long axis (1). 
187. Anterior tip of the preacetabular process of the ilium situated posterior to the anterior tip of the pubic peduncle of the ilium (0), anterior tip of the preacetabular process of the ilium situated anterior to the anterior tip of the pubic peduncle of the ilium (1).

188. Length of the postacetabular process of the ilium between $40 \%$ and $21 \%$ the total length of the ilium (0), postacetabular process of the ilium less than $20 \%$ the total length of the ilium (1), postacetabular process of the ilium greater than $40 \%$ the total length of the ilium (2).

189. Brevis shelf on the ilium oriented vertically (0), brevis shelf on the ilium extends medially in a roughly horizontal plane (1).

190. Ischiac peduncle of the ilium is not supported by a sacral rib (0), ischiac peduncle of the ilium supported by a sacral rib (1).

191. Lateral swelling of the ischiac peduncle of the ilium absent (0), present (1).

192. Pubic peduncle of the ilium more robust than the ischial peduncle and expands in lateral view (0), pubic peduncle of the ilium tapers distally and is smaller than the ischial peduncle (1).

193. Pubis not secondarily supported (0), pubis supported only by sacral rib (1), pubis supported directly by at least one sacral centrum (2).

194. Pubis anteroventrally facing (0), pubis vertically oriented (1), pubis posteroventrally rotated (2).

195. Anterior process of the pubispresent and straight $(0)$, anterior process of the pubis present and dorsally curved (1), anterior process of the pubis absent (2).

196. Angle between prepubic process and pubic shaft greater than $150^{\circ}(0)$, angle less than $100^{\circ}(1)$.

197. Prepubic process of the pubis short and peg shaped (0), prepubic process of the pubis mediolaterally flattened (1), prepubic process of the pubis rod shaped (2), prepubic process of the pubis dorsoventrally flattened (3).

198. Length of the prepubic process of the pubis (measured from the obturator notch) less than $20 \%$ the total length of the ilium (0), prepubic process of the pubis greater than $20 \%$ the total length of the ilium (1).

199. Iliac and pubic peduncles of the ischium continuous, but separated by a fossa (0), iliac and pubic peduncles distinct and separated by a concave surface (1).

200. Pubic peduncle of ischium larger than iliac peduncle $(0)$, peduncles subequal or iliac peduncle larger than pubic peduncle (1).
201. Dorsal margin of ischial shaft straight at midlength in lateral view (0), caudodorsally convex at midlength in lateral view (1), caudodorsally concave at midlength in lateral view (2).

202. Groove on the dorsal edge of the ischium absent $(0)$, present (1).

203. Tab-shaped obturator process absent (0), present (1).

204. Obturator process placed within the proximal $40 \%$ of the ischium (0), obturator process placed within the distal $60 \%$ (1).

205. Ischial shaft flat and bladelike (0), ischial shaft ovoid to subcylindrical (1).

206. Ischial symphysis present along at least $50 \%$ of the ischial shaft (0), ischial symphysis only present distally (1).

207. Enlarged "foot" on the distal end of the ischial shaft absent (0), present (1).

208. Minimum diameter of the femur less than $15 \%$ of total femur length (0), minimum diameter of the femur greater than $15 \%$ of total femur length (1).

209. Femoral shaft straight in anterior view (0), femoral shaft distinctly bowedin anterior view (1).

210. Angle between the neck of the femoral head and the femoral shaftless than or equal to $100^{\circ}(0)$, angle greater than $100^{\circ}(1)$.

211. Necklike constriction under the head of the femur absent (0), present (1).

212. Trench between the greater trochanter and the head of the femur absent (0), present (1).

213. Lateral surface of the greater trochanter of femur convex (0), lateral surface of the greater trochanter flattened (1).

214. Intertrochanteric notch between the lesser and greater trochanters on the femur present $(0)$, absent (1).

215. Dorsal margin of the lesser trochanter of the femur lower than or equal to the height of the greater trochanter (0), dorsal margin of lesser trochanter higher than the height of the greater trochanter (1).

216. Lesser trochanter of femur positioned anterior and medial to greater trochanter $(0)$, lesser trochanter positioned anterior and somewhat lateral to greater trochanter (1).

217. Lesser trochanter of femur consists of a prominent crest $(0)$, lesser trochanter similar in width to the greater trochanter and separated from it by a wide cleft (1), lesser trochanter narrow and closely appressed to the greater trochanter (2).

218. Dorsal margin of the lesser trochanter substantially lower than the head of the femur (0), dorsal 
margin of the lesser trochanter approximately the same height as the head of the femur (1).

219. Fourth trochanter of the femur moundlike $(0)$, fourth trochanter a sharp ridge (1), fourth trochanter pendant shaped (2), fourth trochanter subtriangular (3), fourth trochanter vestigial, consisting of a rugosity or scar (4).

220. Insertion scar of $\mathrm{m}$. caudifemoralis longus on femur extends from fourth trochanter onto medial surface of femoral shaft (0), insertion scar of $m$. caudifemoralis longus widely separated from fourth trochanter and restricted to the medial surface of the femoral shaft (1).

221. Fourth trochanter entirely on the proximal half of the femur $(0)$, fourth trochanter placed at or below midshaft of the femur (1).

222. Anterior intercondylar groove on the distal femur absent (0), present (1).

223. Anterior intercondylar groove of the femur broad, shallow, and V-shaped with the edges of the groove meeting at an angle of greater than $90^{\circ}(0)$, anterior intercondylar groove tight, deep, and $\mathrm{V}$-shaped with edges of the groove meeting at less than a $90^{\circ}$ angle (1), anterior intercondylar groove deep, narrow, U-shaped, and partially enclosed by a slight expansion of the medial condyle (2), anterior intercondylar groove U-shaped and partially enclosed by expansions of both distal condyles (3), anterior intercondylar groove consists of a canal fully enclosed by fusion of lateral and medial condyles (4).

224. Posterior intercondylar groove on the femurfully open (0), medial condyle inflated and at least partially covering the intercondylar groove (1).

225. Posterior intercondylar groove extends less than $1 / 4$ the length of the femur ( 0$)$, posterior intercondylar groove extends greater than $1 / 4$ the length of the femur (1).

226. Femur shorter than or equal to tibia in length (0), femur longer than tibia (1).

227. Tibia triangular in transverse section (0), tibia rounded in transverse section (1).

228. Lateral proximal condyles on the tibia equal in size (0), fibular condyle smaller (1), only one lateral proximal condyle present (2), fibular condyle larger (3).

229. Lateral extension of the tibial posterior flange does not reach fibula $(0)$, lateral extension of the tibial posterior flange extends posterior to the medial margin of the fibula (1), lateral extension of the tibial posterior flange extends posterior to entire distal end of the fibula and calcaneum (2), lateral extension of the tibial posterior flange absent (3).

230. Cnemial crest of tibiastraight (0), cnemial crest arcs anterolaterally (1).

231. Cnemial crest of tibia rounded (0), cnemial crest sharply defined (1).

232. Lateral distal condyle on the femur subequal to the medial distal condyle (0), lateral distal condyle $80 \%-60 \%$ the size of the medial distal condyle (1), lateral distal condyle $59 \%-50 \%$ the size of the medial distal condyle (2), lateral distal condyle $49 \%-40 \%$ the size of the medial distal condyle (3), lateral distal condyle $39 \%-30 \%$ the size of the medial distal condyle (4), lateral distal condyle $29 \%-20 \%$ the size of the medial distal condyle (5).

233. Fibular shaft elliptical or rounded in transverse section at midlength (0), fibular shaft D-shaped in transverse section throughout its length (1).

234. Ascending process of astragalus short (0), ascending process triangular and toothlike (1), ascending process spikelike (2), ascending process relatively large (3).

235. Posterior side of astragalus low (0), high (1).

236. Height of anterior side of astragalus high (0), extends moderately high (1), height of anterior side low (2).

237. Articular surface for fibula on the astragalus covers more than $30 \%$ of the proximal surface (0), articular surface covers less than $30 \%$ of proximal surface of astragalus (1).

238. Angle between the articular facets for the tibia and fibula on the calcaneum greater than $120^{\circ}(0)$, angle less than $120^{\circ}(1)$.

239. Three or more distal tarsals present $(0)$, two or fewer distal tarsals present (1).

240. Medial distal tarsal blocky, thin, and rectangular in proximal view $(0)$, medial distal tarsal round in proximal view (1).

241. Medial distal tarsal does not cover any part of the proximal surface of metatarsal II (0), medial distal tarsal covers at least a portion of the proximal surface of metatarsal II (1).

242. Lateral distal tarsal square shaped (0), kidney shaped (1).

243. Metatarsal I present (0), absent (1).

244. Metatarsal V present (0), absent (1).

245. Metatarsal $\mathrm{V}$ less than $25 \%$ the length of metatarsal III (0), between $25 \%$ and $50 \%$ (1), greater than $50 \%(2)$.

246. Diameter of the midshafts of metatarsals I and V subequal to or greater than metatarsals II-IV (0), 
diameter of the midshafts of metatarsals I and V less than metatarsals II-IV (1).

247. Four functional digits in the pes (0), three functional digits in the pes (1).

248. Phalanges in pedal digit I present (0), absent (1).

249. Unguals on pedal digits II-IV longer than wide (0), approximately as wide as or wider than long (1). 250. Ossified hypaxial tendons along the tail absent (0), present (1).

251. Epaxial ossified tendons absent (0), present (1).

252. Epaxial tendons longitudinally arranged into a single layer (0), arranged in a double-layered lattice (1).

253. Postcranial osteoderms absent (0), present (1).

254. Dermal sculpturing of the skull and/or mandible absent (0), present (1).

255. Premaxillae unfused (0), fused (1). 



\title{
Scientific Publications of the American Museum of Natural History
}

AMERICAN MUSEUM NOVITATES

BULLETIN OF THE AMERICAN MUSEUM of NATURAL History

Anthropological Papers of the American Museum of Natural History

\author{
Publications Committee \\ ROBERT S. VOSS, CHAIR \\ BOARD OF EDITORS \\ JiN MENG, PALEONTOLOGY \\ LORENZO PRENDINI, INVERTEBRATE ZOOLOGY \\ ROBERT S. VOSS, VerTEBRATE ZOOLOGY \\ Peter M. Whiteley, ANTHRopology \\ MANAGING EDITOR \\ MARY KNIGHT
}

Submission procedures can be found at http://research.amnh.org/scipubs

All issues of Novitates and Bulletin are available on the web (http://digitallibrary.amnh.

org/dspace). Order printed copies on the web from:

http://shop.amnh.org/a701/shop-by-category/books/scientific-publications.html

or via standard mail from:

American Museum of Natural History-Scientific Publications

Central Park West at 79th Street

New York, NY 10024

(2) This paper meets the requirements of ANSI/NISO Z39.48-1992 (permanence of paper).

ON THE COVER:THREE SPECIMENS OF THE ORNITHISCHIAN DINOSAUR haya griva, Upper Cretaceous Javkhlant Formation, MongoLIA. IGM 100/2017 (TOP, HOLOTYPE), IGM 100/2019 (MIDDLE), AND IGM 100/2015 (BOTTOM). NOt to SCALE. 